\title{
Tyrosine Phosphorylation linked to Cardiac Arrhythmias via HCN Pacemaker Channel
}

\author{
Yen-Chang Lin \\ West Virginia University
}

Follow this and additional works at: https://researchrepository.wvu.edu/etd

\section{Recommended Citation}

Lin, Yen-Chang, "Tyrosine Phosphorylation linked to Cardiac Arrhythmias via HCN Pacemaker Channel" (2011). Graduate Theses, Dissertations, and Problem Reports. 4747.

https://researchrepository.wvu.edu/etd/4747

This Dissertation is protected by copyright and/or related rights. It has been brought to you by the The Research Repository @ WVU with permission from the rights-holder(s). You are free to use this Dissertation in any way that is permitted by the copyright and related rights legislation that applies to your use. For other uses you must obtain permission from the rights-holder(s) directly, unless additional rights are indicated by a Creative Commons license in the record and/ or on the work itself. This Dissertation has been accepted for inclusion in WVU Graduate Theses, Dissertations, and Problem Reports collection by an authorized administrator of The Research Repository @ WVU.

For more information, please contact researchrepository@mail.wvu.edu. 
Tyrosine Phosphorylation linked to Cardiac Arrhythmias via HCN Pacemaker Channel

Yen-Chang Lin

\begin{abstract}
Dissertation submitted to the
School of Medicine at West Virginia University

in partial fulfillment of the requirements for the degree of
\end{abstract}

Doctor of Philosophy

in

Cellular and Integrative Physiology

\author{
Han-Gang Yu, Ph.D., Chair \\ S. Jamal Mustafa, Ph.D. \\ John Hollander, Ph.D. \\ Pingnian He, M.D., Ph.D. \\ Ming Pei, M.D., Ph.D.
}

\begin{abstract}
Department of Physiology and Pharmacology
Center for Cardiovascular and Respiratory Sciences
\end{abstract}

\title{
Morgantown, West Virginia \\ 2011
}

Keywords: Arrhythmias, HCN channels, Tyrosine phosphorylation, L-type calcium channels 


\begin{abstract}
Tyrosine Phosphorylation linked to Cardiac Arrhythmias via HCN Pacemaker Channel
\end{abstract}

\author{
Yen-Chang Lin
}

Cardiac sudden death (CSD) is the leading cause of death in the United States. Although the causes of CSD are not understood completely, cardiac arrhythmias have been indicated as the major contribution to CSD. The main purpose of this thesis is to investigate the mechanisms underlying cardiac arrhythmias, therefore providing scientific basis for clinical applications in the future to lower the rate of CSD.

My thesis contains four chapters. Chapter 1 aims to examine a novel role of tyrosine phosphorylation in the arrhythmogenesis. To achieve this goal, a rat arrhythmia model was established without structural remodeling, and the Src tyrosine kinase and protein tyrosine phosphatase were evaluated in the induction of cardiac arrhythmias by using electrocardiography (ECG) technique (two manuscripts are in preparation).

In chapter 2, we elucidate the mechanism tyrosine phosphorylation used to modulate the cardiac pacemaker channels that directly contribute to the regulation of heart rate and their potential new role in cardiac arrhythmias ( $\mathrm{J}$ Biol Chem 284: 30433-30440, 2009).

In chapter 3, we explore a novel role of Hyperpolarization-activated, cyclic nucleotide-gated $(\mathrm{HCN})$ channels serving as a regulatory protein to regulate the $\mathrm{Ca}^{2+}$ influx via inactivation of Ltype calcium channel (LTCC) (Am J Physiol Cell Physiol 298(5): C1029-37, 2010).

In chapter 4, the results that have not been published are summarized. These results represented my efforts to explore previously unrecognized mechanisms that underlie the genesis and development of cardiac arrhythmias that predispose the heart to CSD, claiming more than 300,000 lives each year in the US.

Collectively, our results indicate that tyrosine phosphorylation plays a critical role in the regulation of cardiac rhythm, possibly via modulation the activities of HCN pacemaker channels. Furthermore, we also found that $\mathrm{HCN}$ channels can limit the $\mathrm{Ca}^{2+}$ influx via induction of fast inactivation of LTCC. These results not only help us to better understand the mechanisms of cardiac arrhythmias, but can also be applied to the future drug development for the prevention of CSD caused by cardiac arrhythmias. 


\section{Acknowledgements}

Firstly, I would like to express my deepest appreciation to my mentor, Dr. Yu, for giving me the opportunity to accomplish this degree in his lab and always giving me insightful advice both in my research and in my life. Thank you, Dr. Yu. I also would like to thank Dr. Hong Kan for teaching me cathetering and ECG recording, and always encouraging me during the one year I

spent in NIOSH. Thank you, Dr. Kan. Also, I would like to thank Dr. Karen Martin for teaching me confocal image technique, our collaborators for kindly providing us with the plasmids, as well as my lab members, Jiangying Huang, Qi Zhang, and Jing He, for their assistance in conducting biochemical experiments for the projects. I would also like to thank my committee members- Dr. Mustafa, Dr. Hollander, and Dr. Pei, for their time and advice on my dissertation, especially Dr. Pei for giving me his precious suggestions every time during the talks. I also would like to thank Dr. Brock, Dr. Goodman, Dr. Nurkiewicz, Dr. Davis, and Dr. Minnear for their kindness and encouragement, as well as Dr. Patenostro for teaching me how to do a good presentation for teaching. Tammy, Lea Ann, and Claire, I do appreciate your kind help whenever needed. Thank you. Lastly, I would also like to thank my friends: Kim, Megan, Himani, Adam, Phoebe, Dovenia, Maryam, Swati, Kati, Kevin, Shin, Fengping, I-Chang, and Chang-Han. Thank you for your accompany and friendship through these years.

I dedicate my thesis to my beloved family. Words cannot express my gratefulness for your support --- I would like to share all my life with you.

Sincerely, Yen-Chang 05/06/2011 


\section{Table of Contents}

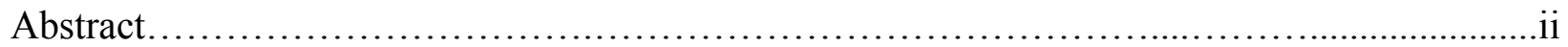

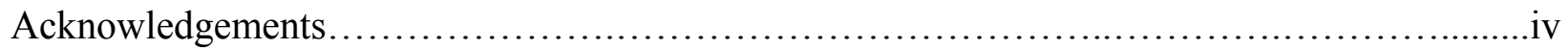

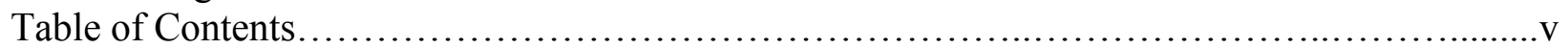

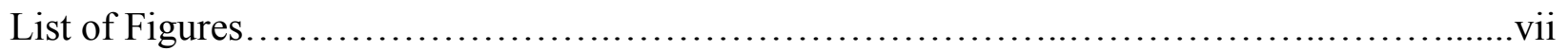

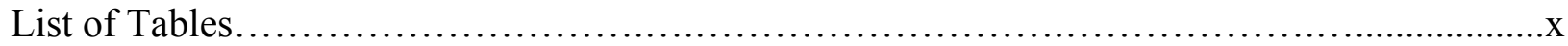

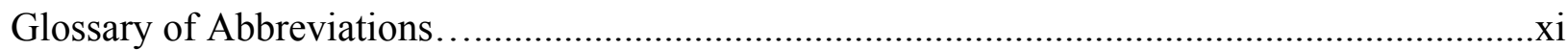

I. General introduction-literature review...........................................

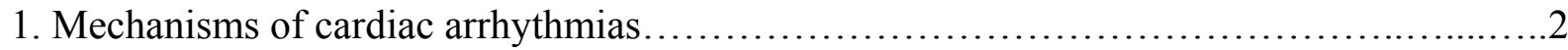

1.1 Abnormal impulse initiation. .................................................

1.2 Abnormal impulse conduction...............................................4

2. Hyperpolarization-activated, cyclic nucleotide-gated (HCN) channels......................4

2.1 Properties of HCN channels................................................

2.2 Tyrosine Phosphorylation of HCN channels in the heart....................................6

$2.3 \mathrm{HCN} 4$ mutants in human and cardiac arrhythmias................................

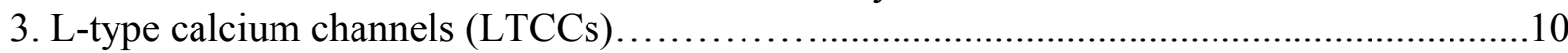

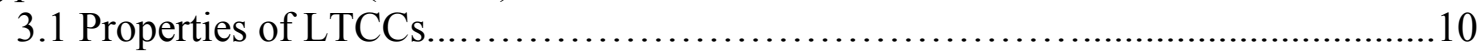

3.2 Role of LTCC inactivation in calcium homeostasis............................ 12

II. General materials and methods .................................................... 13

1. Whole animal preparation and drugs administration................................. 13

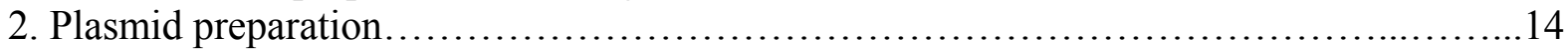

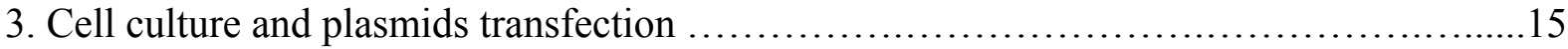

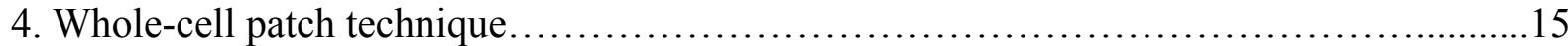

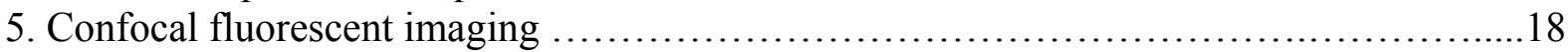

6. Fluorescence recovery after photobleaching (FRAP) ...................................19

III. Chapter 1: Establishment of rat arrhythmia model without structural remodeling for studying

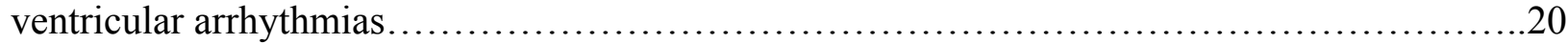

Part I: Inhibition of tyrosine phosphatase activity induced ventricular arrhythmias.........21

Part II: Inhibition of Src kinase activity reduced heart rate and heart rate variability..........30

IV. Chapter 2: Elucidate cellular mechanisms of tyrosine phosphorylation in the regulation of heart rate and cardiac arrhythmias.................................................... 44

Part I: Rescue of a trafficking defective human pacemaker channel via a novel mechanism:

role of Src, Fyn, and Yes............................................................... 45

Part II: Novel mechanism for suppression of hyperpolarization-activated cyclic nucleotidegated pacemaker channels by receptor-like tyrosine phosphatase-alpha....................65

V. Chapter 3: Inactivation of L-type calcium channel modulated by HCN2 channel ............73

VI. Chapter 4: Trafficking mechanism of HCN4 and its mutant and the novel role of HCN2 in modulation of $\mathrm{Ca}^{2+}$ influx........................................................... 100

Part I: Trafficking mechanisms of HCN4 and its mutants identified in patient...........101 
PartII: Association between HCN2 and L-type calcium channels by using fluorescence

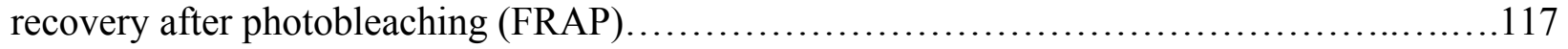

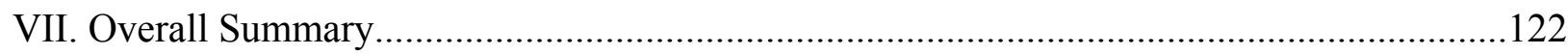

VIII. Literature Cited..................................................................................................130

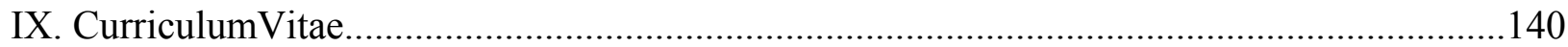

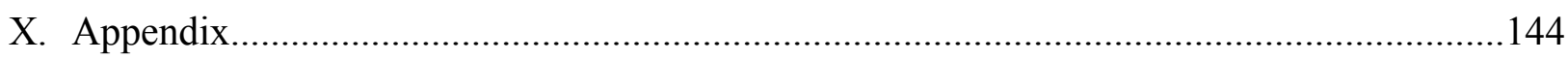




\section{List of Figures}

Chapter 1: Establishment of rat arrhythmia model without structural remodeling for studying ventricular arrhythmias........................................................20

Part I: Inhibition of tyrosine phosphatase activity induced ventricular arrhythmias.............21

Figure 1. Measurements of normal ECG in anesthetized SD rats..................................25 Figure 2. Premature ventricular contraction and fragmented QRS were induced by $\mathrm{Na}_{3} \mathrm{VO}_{4}$ at the

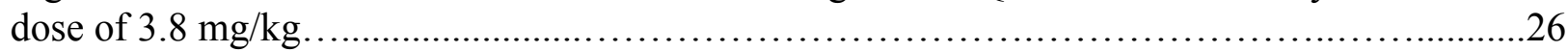

Figure 3. Ventricular tachycardia was induced by $\mathrm{Na}_{3} \mathrm{VO}_{4}$ at the dose of $3.8 \mathrm{mg} / \mathrm{kg} \ldots \ldots \ldots \ldots . .27$

Figure 4. Ventricular fibrillation was induced by $\mathrm{Na}_{3} \mathrm{VO}_{4}$ at the dose of $5.5 \mathrm{mg} / \mathrm{kg} \ldots \ldots \ldots \ldots \ldots .28$

Part II: Inhibition of Src kinase activity reduced heart rate and heart rate variability.............30

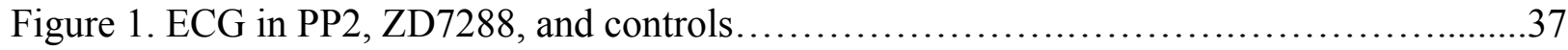

Figure 2. Heart rate variability in PP2, ZD7288, and controls................................38

Figure 3. Heart rate variability analysis in Poincare Plot......................................39

Chapter 2: Elucidate cellular mechanisms of tyrosine phosphorylation in the regulation of heart

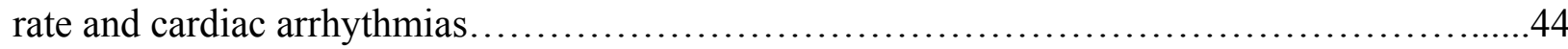
Part I: Rescue of a trafficking defective human pacemaker channel via a novel mechanism: role

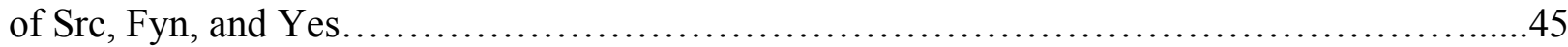

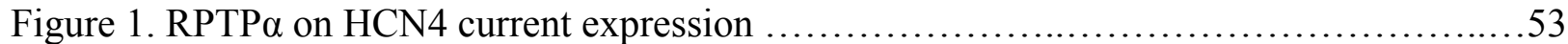

Figure 2. Src/RPTP $\alpha$ on HCN4 cell surface expression ...................................54

Figure 3. Src/Fyn/Yes kinases on current expression of D553N at $-125 \mathrm{mv} \ldots \ldots \ldots \ldots \ldots \ldots \ldots . \ldots 5$

Figure 4. Src/Fyn/Yes kinases on current density and gating of D553N compared with HCN4..56

Figure 5. Src/Fyn/Yes kinases on D553N fluorescence imaging..........................57

Part II: Novel mechanism for suppression of hyperpolarization-activated cyclic nucleotide-gated pacemaker channels by receptor-like tyrosine phosphatase-alpha........................65

Figure 1. RPTP $\alpha$ inhibition of HCN2 current expression.....................................69

Figure 2. RPTP $\alpha$ induced time-dependent inhibition of HCN2 current expression.................70

Chapter 3: Inactivation of L-type calcium channel modulated by HCN2 channel................73

Figure 1. $\mathrm{Ca}^{2+}$-dependent inactivation (CDI) and voltage-dependent inactivation (VDI) of

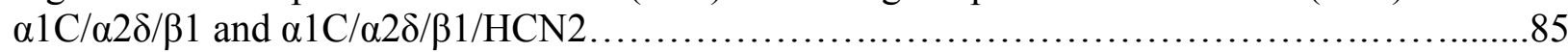

Figure $2 . \alpha 1 \mathrm{C} / \mathrm{HCN} 2$ channel inactivation................................................. 86

Figure 3. Role of calmodulin $(\mathrm{CaM})$ in $\alpha 1 \mathrm{C} / \mathrm{HCN} 2$ channel inactivation........................88

Figure 4. $\alpha 1 \mathrm{C} / \mathrm{HCN} 2$ interaction requires $\mathrm{NH} 2$ terminus of $\mathrm{HCN} 2$ and IQ motif of $\alpha 1 \mathrm{C} \ldots . . . . .89$

Figure 5. Altered L-type $\mathrm{Ca}^{2+}$ channel (LTCC) inactivation in rat hippocampal neurons (HN)

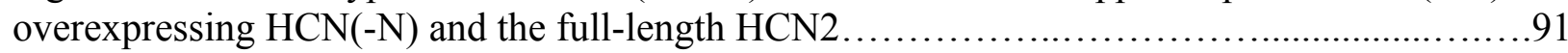

Figure 6. A schematic model depicting the potential roles of HCN2 and $\delta$-subunit in the

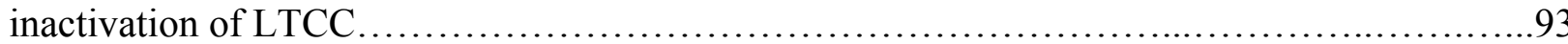

Chapter 4: Trafficking mechanism of HCN4 and its mutant and the novel role of HCN2 in

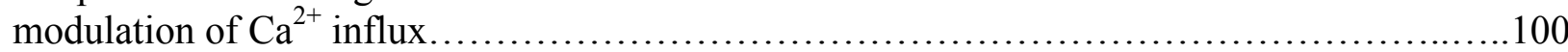

Part I: Trafficking mechanisms of HCN4 and its mutants identified in patient.................101

Figure 1. Colocalization between D553N mutant and the ER maker KKXX..................105

Figure 2. Non-colocalization of wild type HCN4 channels and the ER marker KKXX............106 
Figure 3. Colocalization between wild type HCN4 channels and the membrane dye FM1-43..107 Part II: Association between HCN2 and L-type calcium channels by using fluorescence recovery

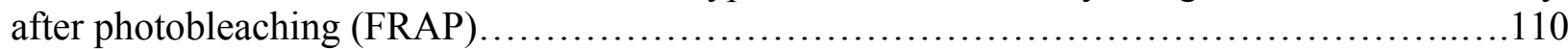

Figure 1. Fluorescence recovery after photobleaching (FRAP) .............................116

Figure 2. Half-time $\left(\mathrm{t}_{1 / 2}\right)$ of HEK 293 cells co-transfected $\alpha$ lC-GFP with HCN2 ................117

Figure 3. Half-time $\left(t_{1 / 2}\right)$ of HEK 293 cells co-transfected N-terminal deletion HCN2 with

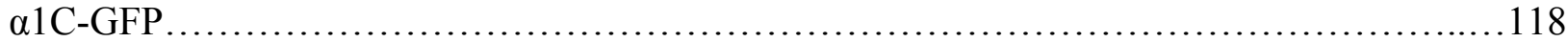

Figure 4. Half-time $\left(\mathrm{t}_{1 / 2}\right)$ of HEK 293 cells co-transfected wild type HCN2-GFP with IQ motif

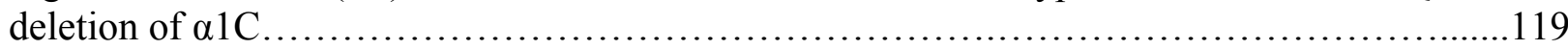

Figure 5. Half-time $\left(\mathrm{t}_{1 / 2}\right)$ of HEK 293 cells co-transfected $\alpha 1 \mathrm{C}$ with HCN2-GFP.................120 


\section{List of Tables}

Chapter 1: Establishment of rat arrhythmia model without structural remodeling for studying

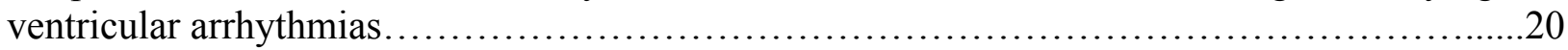

Part II: Inhibition of Src kinase activity reduced heart rate and heart rate variability.............30

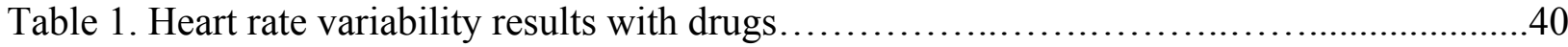

Chapter 2: Elucidate cellular mechanisms of tyrosine phosphorylation in the regulation of heart rate and cardiac arrhythmias...........................................................44 Part I: Rescue of a trafficking defective human pacemaker channel via a novel mechanism: role of Src, Fyn, and Yes............................................................... 45 Table 1. Relative roles of Src, Fyn, and Yes on D553N surface/current expression and activation

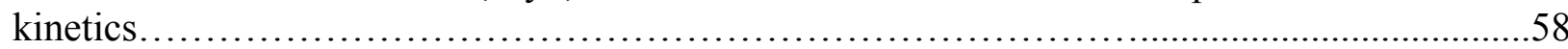




\section{Glossary of Abbreviations}

ANS

AV

$\mathrm{CaM}$

CDI

CNBD

CSD

DAD

EAD

EGF

ECG

FRAP

$\mathrm{HCN}$

HEK

HR

HRV

LTCC

PAO

PVC

RPTP $\alpha$

SA

SD

VDI

VF

VT
Autonomic nervous system

Atrial-ventricular

Calmodulin

Calcium-dependent inactivation

Cyclic nucleotide-binding domain

Cardiac sudden death

Delayed after depolarization

Early after depolarization

Epidermal growth factor

Electrocardiogram

Fluorescence recovery after photobleaching

Hyperpolarization-activated cyclic nucleotide-gated

Human embryonic kidney

Heart rate

Heart rate variability

L-type calcium channel

Phenylarsine oxide

Premature ventricular contraction

Receptor protein tyrosine phosphatase $\alpha$

Sinoatrial

Sprague-Dawley

Voltage-dependent inactivation

Ventricular fibrillation

Ventricular tachycardia 


\section{Introduction}

Cardiac sudden death (CSD) affects approximately 300,000 people in the United Stated every year, and is estimated to account for nearly 50 percent of all death from cardiovascular causes (Myerburg et al., 1997). Major cardiac diseases such as ischemia, heart failure, and cardiac hypertrophy have been reported to cause CSD via ion channel remodeling and changes in ion influx or efflux (Heman et al., 1996; Hynes et al., 2002; Mammarella et al., 2000; Wolfe et al., 1991). However, there are also cases where people, especially young adults, died of sudden CSD in the absence of defective heart structure including hypertrophy (Heman et al., 1996; Hynes et al., 2002; Mammarella et al., 2000; Wolfe et al., 1991). This indicates that our knowledge of CSD is not a complete picture. From a pathological point of view, it is commonly accepted that the underlying cause of CSD is tachycardia-induced arrhythmias (Kannel et al., 1987; Willich et al., 1987). Therefore, to obtain a comprehensive understanding of arrhythmias is the key to understand the causes of CSD. However, the underlying mechanisms of cardiac arrhythmias still remain obscure due to lack of a good animal model system with inducible and controlled real-time ventricular arrhythmias, which the first part of my thesis is focused on.

To provide an ionic basis of cardiac arrhythmias that I observed in the new arrhythmic animal model described in the first part, the second part of this thesis concentrates on two ion channels: Hyperpolarization-activated and cyclic nucleotide-gated $(\mathrm{HCN})$ channels and L-type calcium channels (LTCCs). Ion channels can directly cause diseases. The term "channelopathy" represents the diseases directly caused by the malfunction of the 
channels (Robert et al., 2005). Mutations in the channel proteins or abnormal regulation of the channel contribute to the channelopathy (Hunter et al., 1994; Zhou et al., 2009). In the past decade, numerous mutations in ion channels have been identified in patients and directly linked to a variety of arrhythmias (Dai et al., 2005; Peter et al., 2007). Therefore, my study aims to provide the molecular and cellular basis of how regulation of ion channels of interest (HCN channels and LTCCs) can serve as good strategies for the therapeutic purposes of cardiac arrhythmias.

\section{Mechanisms of cardiac arrhythmias:}

Ion channels are major players to regulate the flow of ions across biological membranes and to maintain cellular homeostasis in the physiological condition. They provide a molecular basis of action potential propagation during the cell excitation. In the resting state, the more negative charges intracellularly make the resting membrane potential around $-70 \mathrm{mV}$ in neurons and $-90 \mathrm{mV}$ in myocytes. Once the action potential arrives, the cells are depolarized by making the intracellular cell membrane more positive via the flow of ions across the channels (Hodgkin et al., 1952; Noble et al., 1960). In heart, the action potential is generated in the "primary pacemaker" sinoatrial node (SA node) in the right atrium and then conducts along atria, the atrial-ventricular node (AV node), Bundles of His, Purkinje fibers, and ventricles. Regulation of SA node rate is critical in understanding of mechanisms, leading to better management of cardiac arrhythmias such as atrial fibrillation and ventricular tachycardia. 


\subsection{Abnormal impulse initiation}

Cardiac arrhythmias are defined as abnormal cardiac electrical properties attributed to two factors: the abnormality in impulse initiation and impulse conduction. Normal impulse initiation is associated with automatic foci determined by the slope of phase 4 depolarization, which establishes the time required to reach threshold potential and generate a spontaneous action potential. The sinus node is the primary pacemaker to initiate and coordinate the electrical impulse which then conducts to the atrium and ventricle. If the sinus node firing rate is slower than atrial cells, the electrical impulse can be initiated and coordinated by atrial cells instead of the sinus node and ectopic activities occurred. The function of $I_{\mathrm{f}}$ currents (mediated by $\mathrm{HCN}$ channels) contributed to the phase 4 of sinus node or atrial or Purkinje action potential and make the "U" turn of the maximal diastolic potential. However, when the slope of phase 4 is accelerated by the increase of $\mathrm{HCN}$ channel expressions, the ectopic impulse or the tachycardia induced arrhythmias will be generated (Hoffman et al., 1981). Cardiac remodeling caused by atrial tachycardia or heart failure (failing ventricles) has been demonstrated to be associated with the enhancement of HCN channel expressions (Nattel S et al., 2007).

Different from the mechanism in phase 4, delayed after depolarizations (DADs) and early after depolarizations (EADs) are caused by abnormalities in cellular $\mathrm{Ca}^{2+}$ handling. DADs are represented by spontaneous hump-shaped depolarizations after repolarization, which will increase more $\mathrm{Ca}^{2+}$ load and trigger sustained tachycardia (Chen et al., 2001; Coutu et al., 2006; Stambler et al., 2003). EADs occur when action potentials become abnormally prolonged due to increased $\mathrm{Ca}^{2+}$ influx via LTCCs and generate abnormal 
depolarization at plateau phases, which are characterized in Purkinje fibers and long-QT syndrome (Burashnikov et al., 2003; Nattel et al., 1988; Satoh et al., 1998).

\subsection{Abnormal impulse conduction}

Abnormal impulse conduction causes reentrant excitation. Reentry occurred when an ectopic impulse was blocked by refractory tissues while propagating in one direction and will then propagate in the other direction where the tissues have faster recovering from refractory periods. For the maintaining of reentry, the time of conducting has to be longer than the refractory period in the circuit (to make sure the impulse can re-excite all points of the entire circuit). The time of conduction is determined by conduction velocity and circuit path length. Slower conduction velocity and longer circuit path length create the environment in which reentry can be maintained. Refractory period of action potential is also a critical determinant for reentry. Short refractory period increases the opportunity that tissues are available to be reactivated by the reentering pulse (Hoffman et al., 1981). Previous studies have demonstrated that atrial remodeling caused by ischemia or heart failure increases the risk of reentrant excitation by increase of circuit path spaces and decrease of refractory periods (For example, decrease inward calcium currents via LTCCs), which potentially results in atrial fibrillation (Cha et al., 2006; Yue et al., 1997).

\section{HCN channels}

\subsection{Properties of HCN channels}

Hyperpolarization-activated, cyclic nucleotide-modulated $(\mathrm{HCN})$ channels are the major contributor to SA node pacemaker activity (Brown et al., 1982; DiFrancesco, 1993; 
Irisawa et al., 1978). Exploring the new mechanisms that control the HCN channel properties by tyrosine phosphorylation constitutes the majority of my thesis work.

HCN channels form tetramers on the cell membrane and each subunit consists of six transmembrane domains including the voltage sensor S4 domain, P loop with the GYG motif for the selectivity of the $\mathrm{K}^{+}$ion flow, and the cyclic nucleotide-binding domain (CNBD) on the C-terminus (Fig 1). There are four isoforms (HCN1-4) of HCN channels. Despite their similarity in structure, different $\mathrm{HCN}$ isoforms do not express uniformly in the heart. HCN4 is the dominant isoform in SA node whereas HCN2 is mainly expressed in ventricles. HCN1 transcripts are expressed at low levels in SA node, and cannot be detected in ventricles (Shi et al., 1999).

Unlike most other voltage-gated ion channels that are activated by membrane depolarization, the $\mathrm{Na}^{+} / \mathrm{K}^{+}$ permeable $\mathrm{HCN}$ channels can only be activated by membrane hyperpolarization, generating a voltage- and time- dependent inward current, called $I_{\mathrm{f}}$ in heart and $I_{\mathrm{h}}$ in neurons. $I_{\mathrm{f}}$ contributes

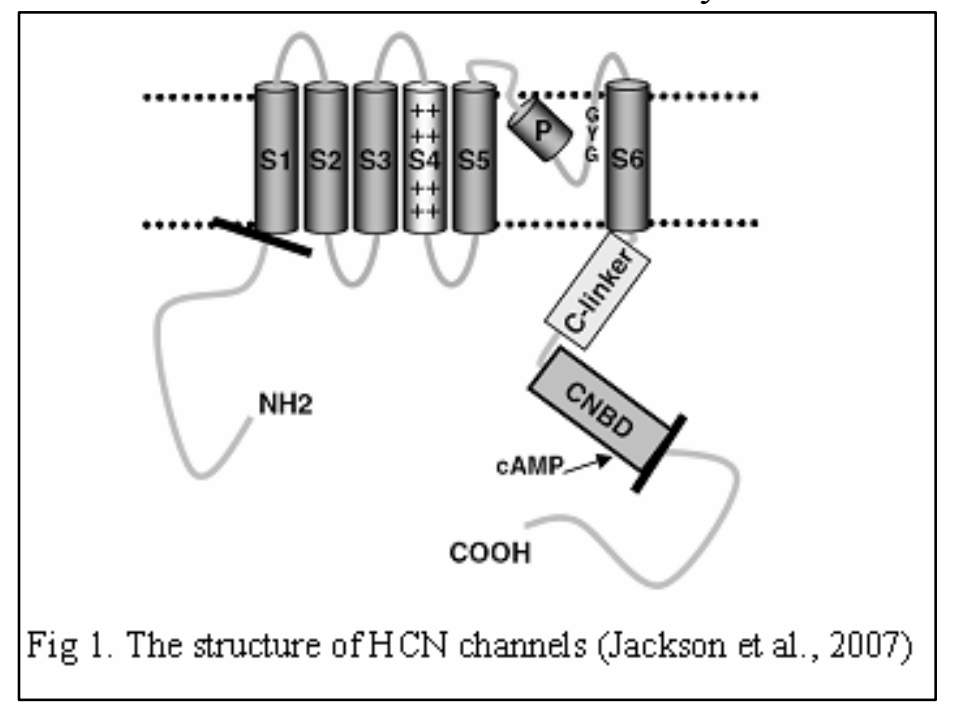
to the termination of the repolarization and generation of the diastolic depolarization to the threshold for firing an action potential (Brown et al., 1982; DiFrancesco, 1993; Irisawa et al., 1978).

HCN channels are regulated by autonomic nervous system. The activation of $\beta$ adrenergic receptors by adrenaline (i.e. norepinephrine) or isoproterenol can cause the 
increase of intracellular cAMP. In a phosphorylation-independent regulation manner, cAMP can interact with $\mathrm{HCN}$ channels at the cyclic nucleotide-binding domain (CNBD) on their C-terminal tail. This triggers conformational change of $\mathrm{HCN}$ channels, resulting in the net positive charge influx, to generate voltage and time dependent inward currents. As a result, the activation curve will shift to more positive voltages (increase of the channel sensitivity in response to the voltage stimulation), contributing to an increase in the heart rate (Accili et al., 1997; Altomare et al., 2001; DiFrancesco et al., 1999).

On the contrary, the activation of muscarinic acetylcholine receptors (type II) by acetylcholine can cause the decrease of cAMP and make activation curve shift to more negative voltages (decrease of the channel sensitivity in response to the voltage stimulation), leading to an decrease in the heart rate for physiological effect (Accili et al., 1997; Altomare et al., 2001; DiFrancesco et al., 1999).

\subsection{Tyrosine Phosphorylation of HCN channels in the heart}

One of the important regulations of HCN channels is via tyrosine phosphorylation. In 2000 , the $I_{\mathrm{f}}$ has been found to be reduced by approximately $30 \%$ after applying genistein, a tyrosine kinase inhibitor, with epidermal growth factor (EGF) when compared with EGF alone in rabbit sinus node myocytes. In accordance with the results in rat ventricular myocytes, not only was $I_{\mathrm{f}}$ enhanced, but its activation threshold was also positively shift by nearly $+20 \mathrm{mv}$ after washout of genistein, demonstrating that tyrosine phosphorylation is critical in the regulation of $\mathrm{HCN}$ channels function (Wu et al., 2000).

To further investigate the phosphorylation-related regulation of the different subtypes of HCN channels, genistein was applied in Xenopus Oocytes overexpressing HCN1, HCN2, 
or HCN4. It has been demonstrated that the conductance of HCN2 and HCN4 were both reduced; whereas $\mathrm{V}_{1 / 2}$ (the voltage where the $50 \%$ channels can be activated) of $\mathrm{HCN} 2$ but not HCN4 was shifted to more negative charge after applying genistein. However, neither the conductance nor $\mathrm{V}_{1 / 2}$ was affected by genistein for $\mathrm{HCN} 1$ channels, suggesting that different subtypes of HCNs respond differently to tyrosine phosphorylation. These results strongly indicate that $\mathrm{HCN}$ channels, especially HCN2 and HCN4, can be tightly regulated by tyrosine phosphorylation (Yu et al., 2004).

To search for the intracellular tyrosine kinases that can provide tyrosine phosphorylation regulation of $\mathrm{HCN}$ channels, tyrosine kinase Src was studied. The Src family is composed of nine members, three of which are ubiquitously expressed in heart- Src, Yes, and Fyn. Structurally, these proteins are highly homologous and consist of an aminoterminal site, $\mathrm{SH}$ domains, and a carboxyl-terminal regulatory region. Functionally, they can phosphorylate the proteins in the signal transduction cascade (Thomas et al., 1997). Interestingly, previous study indicated that Src529, a constitutively active Src tyrosine kinase, was capable of enhancing current densities and positively shifting the $\mathrm{V}_{1 / 2}$ and activation threshold after co-expression with HCN4 channels in HEK 293 cells. In contrast, Src296, a dominant negative Src tyrosine kinase, caused the reduction of current densities and decreased the gating properties of HCN4 channels. This suggests that the regulation of $\mathrm{HCN}$ is probably via Src-mediated tyrosine phosphorylation (Arinsburg et al., 2006).

In 2008, by using a specific Src kinase inhibitor, PP2 (4-amino-5-(4-chlorophenyl)-7-(tbutyl)pyrazolo[3,4- $d]$ pyramidine), it has been revealed that the conductance of HCN4 
channels was significantly decreased and the activation threshold was also negatively shifted due to the reduction of tyrosine phosphorylation. Immunoprecipitation results demonstrate the possible association between the kinase Src and HCN4 channels. By employing PP2 and whole-cell patch clamp technique, our lab also found that Y531 is the main residue for the Src-mediated tyrosine phosphorylation (Li et al., 2008).

Recently, a tyrosine phosphatase inhibitor, phenylarsine oxide (PAO), has also been demonstrated to enhance the $I_{\mathrm{f}}$ density of $\mathrm{HCN} 4$ channels and positively shift the threshold of activation about $+40 \mathrm{mV}$ in adult ventricular myocytes. To further identify the specific tyrosine phosphatase that contributed to the PAO effects on $I_{\mathrm{f}}$, we investigated the potential modulation of receptor protein tyrosine phosphates (RPTP). Receptor Protein Tyrosine Phosphatase $\alpha$ (RPTP $\alpha)$, a member of receptor-type tyrosine phosphatase, was cotransfected with HCN2 or HCN4 channels in HEK 293 cells. Interestingly, $\mathrm{HCN} 2$ or HCN4 currents were totally eliminated by RPTP $\alpha$ two days after transient co-transfection HCN2 or HCN4 with RPTP $\alpha$. Biochemical approach further indicates that tyrosine dephosphorylation by RPTP $\alpha$ impairs membrane trafficking of HCN2 channels, possibly due to an association between RPTP $\alpha$ and HCN2 channels. This may explain why the $\mathrm{I}_{\mathrm{f}}$ density was significantly reduced in rat ventricle by applying a tyrosine phosphatase inhibitor PAO (Huang et al., 2008).

\subsection{HCN4 mutants in Human Cardiac Arrhythmias}

HCN4 channels are closely related to the initiation of cardiac pacemaker activity. Defects in the HCN4 channels including gene knockout have caused death in embryo, and severe cardiac arrhythmias (Stieber et al., 2003). 
Several gene mutations of HCN4 channels have been reported to be implicated to the cardiac arrhythmia and to impair the cardiac function such as HCN4-573X, S672R, G480R, and D553N mutations.

HCN4-573X is a single base pair mutation, which leads to a premature stop codon resulting in the protein truncation of C-terminal including the CNBD domain of the channels. This leads to loss of interaction with cAMP and alteration of deactivation kinetics in channels (Schulze et al., 2003).

S672R missense mutation is located in the $\beta$ roll of the CNBD domain. This mutant channel causes a shift of the activation threshold towards the more hyperpolarizing direction (which reduces $I_{\mathrm{f}}$ in sinoatrial node at a given hyperpolarized voltage), therefore slows down the heart rate. This mutant also decreases the sensitivity of the channels in response to the membrane hyperpolarization. However, the cAMP modulation is not changed in this single base mutation (Milanesi et al., 2006).

G480R is located in the pore region of HCN4 channels and has been reported to be associated with reduction of pacemaker currents and HR. Previous studies by using Xenopus oocytes and HEK293 cells revealed that reduction of current expression is attributed to a more negative voltage dependence of channel activation as well as decrease of membrane expression of this mutant (Nof et al., 2007).

The relevant one to my own study is the D553N missense mutation in the C-linker of HCN4 channels. This mutant was found in a patient who suffers from a severe cardiac arrhythmia including sinus node dysfunction-induced bradycardia (lower heart rate), long 
QT syndrome, and ventricular tachycardia polymorphisms indicated by electrocardiogram (ECG) (Ueda et al., 2004). By employing the electrophysiology and imaging, further investigation also implicated that this mutant is a dominant negative mutant and causes the membrane trafficking defect of the channels. Not only the current densities are significantly reduced, this mutant can also alter the kinetics of activation and deactivation of the channel. As the trafficking defect of this mutant results in cardiac arrhythmia, my main goal is to test the hypothesis that the Src kinases-mediated tyrosine phosphorylation can rescue the defective trafficking of this mutant.

\section{L-type calcium channels (LTCCs):}

\subsection{Properties of LTCCs:}

LTCCs are pivotal to control the extracellular calcium influx into the cell and in turn to regulate the calcium-dependent physiological functions of the cell. LTCCs consist of the pore-forming $\alpha 1$ subunit with several accessory subunits such as $\alpha 2 \delta, \beta$, and $\gamma$ subunits surrounding this pore subunit (Arikkath et al., 2003; McCleskey et al., 1994; Tsien et al., 1995; Yasuda et al., 2004). The pore-forming $\alpha 1$ subunit has four repeats and each one has six transmembrane domains, which is capable of mediating the biophysical and pharmacological properties of the channels, while its accessory subunits are critical to regulate the gating and trafficking of the channels (Arikkath et al., 2003; Yasuda et al., 2004). It is generally accepted that LTCCs require association of the pore-forming $\alpha 1$ subunit with its accessory $\beta$ and $\alpha 2 \delta$ subunits to generate the functional calcium currents in response to depolarization. 
In the heart, cardiac contractions of the atrium and ventricle occur via activation of LTCCs. In a resting condition, the intracellular calcium concentration is maintained at approximately $0.1 \mu \mathrm{M}$, which is nearly $1 / 10000$ of the extracellular free calcium concentration (1mM) (Bers, 2002). During action potential, in cardiomyocytes calcium enters the cell through depolarization-activated calcium channels as inward calcium current, which can trigger calcium release from the sarcoplasmic reticulum (calcium induced calcium release), and then switches on the contractile machinery (Bers, 2000). Therefore, the physiological function of the heart is controlled by this excitationcontraction coupling (EC-coupling) (Bers, 2002).

Abnormal pacemaking or conduction can lead to cardiac arrhythmia, such as long QT syndromes (LQTS), a precursor of sudden cardiac arrest. On the other hand, once the EC coupling is impaired by disruptive $\mathrm{Ca}^{2+}$ homeostasis, the ventricular pacing will not be controlled by sinus node rate, making the ventricle prone to arrhythmias (Splawski et al., 2004; 2005).

Long QT syndrome is caused by either an increase in inward currents (causing the prolonged plateau phase of ventricular action potential) or a decrease of outward currents (causing the delayed repolarization of plateau phase of ventricular action potential) (Morita et al., 2008). As L-type calcium current is one of the main inward currents (Splawski et al., 2004; 2005), it is commonly believed that by modulating the inactivation of LTCCs, $\mathrm{Ca}^{2+}$ influx can be controlled, thus rescue diseases like long QT. 


\subsection{Role of LTCC inactivation in calcium homeostasis}

Calcium influx regulation is critical for the normal function of cardiac cells. The amount of $\mathrm{Ca}^{2+}$ ions can be delicately regulated by the activation and inactivation of calcium channels. The inactivation process of LTCCs has been extensively studies in Xenopus oocytes or HEK 293 cells (Kim et al., 2004; Zuhlke et al., 1998). The C-terminus of $\alpha 1$ subunit locates intracellularly and contains motifs including the IQ motif and EF hand. Although the potential inactivation mechanisms for LTCCs are controversial, it is generally believed that the IQ motif and the EF hand upstream the IQ motif on the $\alpha 1$ subunit C-terminal region are where calmodulin $(\mathrm{CaM})$ is pre-associated with; and these domains combined with I-II loop are responsible for mediating the calcium-dependent fast inactivation (CDI) (using $\mathrm{Ca}^{2+}$ as the charge carrier) as well as the voltage-dependent slow inactivation (VDI) (using $\mathrm{Ba}^{2+}$ as the charge carrier) of the calcium channels (Cens et al., 2006; Kim et al., 2004; Pitt et al., 2001; Zuhlke et al., 1998).

Calcium influx is a pivotal second messenger for intracellular signal transduction to maintain cell physiological function such as cell contraction, neurotransmitter release, and cardiac cell excitability (Adams et al., 1986; Dewaard et al., 1998; Forder et al., 1985). On the other hand, high intracellular calcium concentration is also toxic and causes cell damage, which has been demonstrated in myocardial ischemia (Fleckenstein et al., 1974) and muscle dystrophy (Wrogemann et al., 1976). Moreover, the abnormal calcium influx is directly linked to cardiac arrhythmias (Novotny et al., 1986; Williams et al., 1984). In my thesis work, I identified a novel role of HCN channel to control inactivation of LTCCs. 


\section{General materials and methods}

\section{Whole animal preparation and drugs administration}

$2 \%$ isoflurane mixed with oxygen at the flow rate of 2 liter/min inhaled by adult SpragueDawley (SD) rats was used for anesthetization in accordance with Animal Care and Use Committee guidelines for implantation of catheters.

The catheter (polyurethane, 1 French size) was inserted into the jugular vein for different doses of drug administration. ECG was measured by using two leads vector. The arterial catheter was connected to a heparin filled pressure transducer for at least 20 minutes before measurements are made.

In part I of chapter 1 , different doses of $\mathrm{Na}_{3} \mathrm{VO}_{4}$ were applied for the induction of ventricular arrhythmias. At the beginning, $3.8 \mathrm{mg} / \mathrm{kg}$ of $\mathrm{Na}_{3} \mathrm{VO}_{4}$ was administrated via the catheter injection which catheter has been placed in jugular vein to induce arrhythmias. For more severe arrhythmias induction, a relatively higher dose of $\mathrm{Na}_{3} \mathrm{VO}_{4}$ $(5.5 \mathrm{mg} / \mathrm{kg})$ was applied.

In part II of chapter 1, different doses of PP2, ZD7288, PP3, and isoproterenol were applied via jugular vein to see effects on HRV. High dose of PP2 (0.3 mg/kg) or ZD7288 $(0.7 \mathrm{mg} / \mathrm{kg})$ as well as low dose of PP2 or ZD7288 $(0.2 \mathrm{mg} / \mathrm{kg})$ were applied whereas the dose of $0.2 \mathrm{mg} / \mathrm{kg}$ was applied in PP3 (negative control of PP2). For isoproterenol, 0.4 $\mu \mathrm{g} / \mathrm{kg}$ was applied as the positive control. 
The data were recorded and analyzed using cardiovascular continuous monitoring software (PowerLab/4SP, ADInstruments, Colorado Springs, CO). Values for hemodynamic measures are derived by $1 \mathrm{KHz}$ sampling rate. All the results are repeated by at least $10 \sim 12$ times $(\mathrm{n}=10 \sim 12)$.

\section{cDNA plasmid preparation}

For HCN4 and HCN4-D553N cDNA plasmids, the human version of HCN4-pcDNA1.1 was kindly provided by Dr. U. B. Kaupp and subcloned into pcDNA3.1 vector. HCN4D553N-pcDNA3 was made by substituting aspartic acid with asparagine using PCR. HCN4-D553N-DsRed was made by subcloning HCN4-D553N into the DsRed vector. RPTP $\alpha$ was a generous gift from Dr. Jan Sap (University of Copenhagen, Copenhagen, Denmark). The constitutively active form of Fyn, FynY531F, in pcDNA3.1 vector, was kindly provided by Dr. Shigeru Kanda (Nagasaki University, Nagasaki, Japan). The constitutively active form of Yes (YesY537F) was kindly provided by Dr. Arkadiusz Welman (Edinburgh Cancer Research Center), and we subcloned it into the pcDNA3.1 vector. Src529 (SrcY529F) was purchased from Upstate Biotechnology (Millipore). For simplicity, we also use Src, Fyn, and Yes and Src529, Fyn531, and Yes537 in the text for interchangeable use with the constitutively active form of each kinase: SrcY529F, FynY531F, and YesY537F.

For HCN2 DNA plasmids, mouse $\mathrm{HCN} 2$ cDNA in an oocyte expression vector, pGH, was initially obtained from Drs. Bina Santoro/Steve Siegelbaum (Columbia University). We subcloned it into the EcoRI/XbaI sites of pcDNA3.1 mammalian expression vector 
(Invitrogen) for functional expression in mammalian cells. RPRP $\alpha$ inserted in pRK5 vector was kindly provided by Dr. Jan Sap (University of Copenhagen, Denmark).

\section{Cell culture and plasmids transfection}

For the study of HCN currents, HEK293 cells were grown in Dulbecco's modified Eagle's medium (Invitrogen), supplemented with 10\% fetal bovine serum, $100 \mathrm{IU} / \mathrm{ml}$ penicillin, and $100 \mathrm{~g} /$ liter streptomycin. Cells with $50-70 \%$ confluence in 6-well plates were used for plasmid transfection (2-5 $\mu \mathrm{g}$ for each plasmid transfection) using Lipofectamine2000 (Invitrogen). For the study of LTCC inactivation, an HEK293 cell line stably expressing $\alpha 2 \delta / \beta 1$ was kindly provided by Dr. Richard Tsien (Stanford University). Culture of these cells was similar to that described above except for addition of antibiotics, G418 (0.5 $\mathrm{mM} / \mathrm{ml}$ ) and $5 \mathrm{mM} \mathrm{L}$-glutamine, to the DMEM. Primary embryonic day 18 rat hippocampus neurons were used for studying the interaction between $\alpha 1 \mathrm{C}$ and $\mathrm{HCN} 2$. Neuron culture and plasmid transfection were completed following the instruction manual (Invitrogen).

\section{Whole-cell patch technique}

For recording $I_{\mathrm{HCN} 4}$, day $1(24-30 \mathrm{~h})$ up to day $4(90-98 \mathrm{~h})$ post-transfection HEK293 cells with green fluorescence were selected for patch clamp studies. The HEK293 cells were placed in a Lucite bath in which the temperature was maintained at $25 \pm 1{ }^{\circ} \mathrm{C}$ by a temperature controller (Cell MicroControls). $I_{\mathrm{HCN} 4}$ currents were recorded using the whole cell patch clamp technique with an Axopatch-200B amplifier. The current amplitude of HCN4 or D553N current is defined as the amplitude of the onset timedependent inward current elicited by the hyperpolarizing pulse, excluding the instant 
jump at the beginning of the pulse. The current density is the current amplitude divided by the cell capacitance measured in each cell studied. The pipettes had a resistance of 2-4 $\mathrm{M} \Omega$ when filled with internal solution: $6 \mathrm{mM} \mathrm{NaCl}, 130 \mathrm{mM}$ potassium aspartate, $2 \mathrm{mM}$ $\mathrm{MgCl}_{2}, 5 \mathrm{mM} \mathrm{CaCl}, 11 \mathrm{mM}$ EGTA, and $10 \mathrm{mM}$ HEPES; $\mathrm{pH}$ was adjusted to 7.2 by $\mathrm{KOH}$. The external solution contained $120 \mathrm{mM} \mathrm{NaCl}, 1 \mathrm{mM} \mathrm{MgCl} 2,5 \mathrm{mM}$ HEPES, 30 $\mathrm{mM} \mathrm{KCl}, 1.8 \mathrm{mM} \mathrm{CaCl} 2 ; \mathrm{pH}$ was adjusted to 7.4 by $\mathrm{NaOH}$. The $I_{\text {to }}$ blocker, 4aminopyridine $(2 \mathrm{mM})$, was added to the external solution to inhibit the endogenous transient potassium current, which can overlap with and obscure $I_{\mathrm{HCN} 4}$ tail currents recorded at $+20 \mathrm{mV}$. The data were acquired by CLAMPEX and analyzed by CLAMPFIT (pClamp 8; Axon).

For $I_{\mathrm{HCN} 4}$ analysis, the data are shown as the means \pm S.E. The threshold activation of $I_{\mathrm{HCN} 4}$ is defined as the first hyperpolarizing voltage at which the first time-dependent inward current can be observed. Student's $t$ test was used for statistical analysis with $p<$ 0.05 being considered statistically significant. Time constants were obtained by using Boltzmann best fit with one exponential function on current traces that reach steady state. HCN4 activates slowly, and the cells would not tolerate pulses sufficiently long to reach the steady state. We therefore used the following approach to obtain an accurate estimate of the steady state activation (Arinsburg et al., 2006). The onset current traces were fitted with a single exponential function to $30-40 \mathrm{~s}$ to allow estimates of steady state current levels. The fitted current amplitudes were then divided by the driving force (the difference between test pulses and the reversal potential that was measured in each cell) to obtain the conductance at each test pulse. The activation curves were constructed by 
normalizing the conductance to its maximal value in response to the most negative test pulse.

For recording $I_{\mathrm{HCN} 2}$, day $1(24-30 \mathrm{~h})$ up to day $4(90-98 \mathrm{~h})$ post-transfection, HEK293 cells with green fluorescence were selected for patch clamp studies. The HEK293 cells were placed in a Lucite bath in which the temperature was maintained at $25 \pm 1{ }^{\circ} \mathrm{C}$ by a temperature controller (Cell MicroControls, Virginia Beach, VA).

$I_{\mathrm{HCN} 2}$ were recorded using the whole-cell patch clamp technique with an Axopatch-200B amplifier. The pipettes had a resistance of $2-4$ megohms when filled with internal solution (mM) as follows: $\mathrm{NaCl} 6$, potassium aspartate $130, \mathrm{MgCl}_{2} 2, \mathrm{CaCl}_{2}$ 5, EGTA 11, and HEPES 10; $\mathrm{pH}$ adjusted to 7.2 by $\mathrm{KOH}$. The external solution contained (mM) the following: $\mathrm{NaCl} 120, \mathrm{MgCl}_{2}$ 1, $\mathrm{HEPES} 5, \mathrm{KCl} 30, \mathrm{CaCl}_{2} 1.8$, and $\mathrm{pH}$ was adjusted to 7.4 by $\mathrm{NaOH}$. The $I_{\text {to }}$ blocker, 4-aminopyridine ( $2 \mathrm{mM}$ ), was added to the external solution to inhibit the endogenous transient potassium current, which can overlap with and obscure $\mathrm{I}_{\mathrm{HCN} 2}$ tail currents recorded at $20 \mathrm{mV}$. Data were acquired by CLAMPEX and analyzed by CLAMPFIT (pClamp 8, Axon Instruments).

For $I_{\mathrm{HCN} 2}$ analysis, data are shown as mean \pm S.E. The threshold activation of $I_{\mathrm{f}}$ is defined as the first hyperpolarizing voltage at which the time-dependent inward current can be observed. Student's $t$ test was used for statistical analysis with $p<0.05$ being considered as statistically significant. Time constants were obtained by using Boltzmann best fit with one exponential function on current traces that reach steady state. 
For the study of LTCC inactivation, patch-clamp recordings are the same as previously reported (Huang et al., 2008). The pipettes had a resistance of 2-5 M $\Omega$ when filled (in $\mathrm{mM}$ ): $108 \mathrm{CsCl}, 4 \mathrm{MgCl}_{2}, 9$ EGTA, and 9 HEPES. The bath solution contained (in mM) $2 \mathrm{BaCl}_{2}, 1 \mathrm{MgCl}_{2}, 10$ HEPES, 40 tetraethylammonium, 10 glucoses, and $65 \mathrm{CsCl}$. Both solutions were adjusted with $\mathrm{CsOH}$ to $\mathrm{pH}$ 7.2. Unless specifically mentioned in the text, the $\mathrm{Ba}^{2+}$ currents $\left(I_{\mathrm{Ba}}\right)$ were recorded at $+10 \mathrm{mV}$ from a holding potential of $-90 \mathrm{mV}$. $I_{\mathrm{Ba}}$ current inactivation was sometimes normalized for better comparison of kinetics in different combinations of plasmids coexpressed in different HEK-293 cells. Normalization is indicated without vertical scales; where vertical scales are present, $\mathrm{P} / 4$ protocols were used to remove the leak currents. The capacitive currents were minimized with $75-85 \%$ series resistance compensation.

\section{Confocal fluorescent imaging}

HEK293 cells transfected with HCN4-DsRed or HCN4-DsRed-D553N or ER marker KKXX or stained by FMI34 (cell membrane dye) were incubated on coverslips and fixed in $4 \%$ paraformaldehyde/PBS for $15 \mathrm{~min}$ and then washed with PBS (10 $\mathrm{mM}$ phosphate buffer, $150 \mathrm{mM} \mathrm{NaCl}, \mathrm{pH}$ 7.4) for 5 min for three times, followed by blocking in $1 \%$ bovine serum albumin/PBS, pH 7.4, for 60 min. After washing six times in PBS, the coverslips were mounted on slide glasses using Fluoromount G (Southern Biotechnology). The cells were imaged by a LSM510 confocal microscopy using a PlanNeofluar $40 \times / 0.75$ objective or a Plan-Apochromat $63 \times / 1.4$ Oil differential interference contrast M27 objective. For DsRed imaging, a 1.2-milliwatt 543-nm HeNe laser was used for excitation, and a 560-615-nm BP emission filter was used for emission. 
For hippocampal neurons, live-cell images of hippocampal neurons were visualized using an inverted Nikon TE2000S epifluorescent microscope with Prior filter wheels and a Photometrics Coolsnap HQ charge-coupled device camera.

\section{Fluorescence recovery after photobleaching (FRAP)}

HEK 293 cells transfected with different combinations of plasmids were placed under LSM510 microscope and stimulated by the laser beam. 480nm was used for GFP. Briefly, FRAP parameters in Capture Preferences was set followed by searching a region to be FRAPed using Focus Window (Eric et al., 2001; Brian et al., 2005). The region of interests was then photobleached by Laser beam. The data were then analyzed by Igor software for the calculation of decay time. 


\section{Chapter1}

\section{Establishment of a Rat Arrhythmia Model without Structural Remodeling for Studying Ventricular Arrhythmias}

The goal of the work presented in this chapter is to explore the potential role of tyrosine phosphorylation in cardiac arrhythmias. Two parts are included. Part I summarizes the role of tyrosine phosphorylation in ventricular arrhythmias. Part II summarizes the role of tyrosine phosphorylation in heart rate variability. 


\title{
Part I: Inhibition of Tyrosine Phosphatase activity induces ventricular arrhythmias
}

\begin{abstract}
Using a small molecule, sodium orthovanadate $\left(\mathrm{Na}_{3} \mathrm{VO}_{4}\right)$ to inhibit the cellular tyrosine phosphatase activity, we have reproducibly detected several major ventricular arrhythmias. At the dose of $3.8 \mathrm{mg} / \mathrm{kg}$, frequent premature ventricular contractions (PVCs) can be induced leading to ventricular tachycardia (VT). Fragmented QRS, a strong marker of abnormal conduction in the ventricle, also appeared. At the higher dose $(5.5 \mathrm{mg} / \mathrm{kg})$, the most severe ventricular arrhythmia, ventricular fibrillation, occurred.

Our goal is to develop a rat model with controllable occurrence of ventricular arrhythmias without cardiac structural remodeling, providing a reliable small animal model to study the novel mechanisms of ventricular arrhythmias and to develop new antiarrhythmic drug for treatment of patients without structural heart disease.
\end{abstract}




\section{Introduction}

Major heart diseases (ischemia, hypertrophy, heart failure) are associated with ventricular arrhythmias leading to sudden cardiac death (Hernan et al., 1996; Hynes et al., 2002; Mammarella et al., 2000; Wolfe et al., 1991). Ventricular arrhythmias also cause cardiac death without cardiac structural remodeling. The underlying mechanisms are unknown, and are hard to study due to lack of a good animal model with inducible and controlled ventricular arrhythmias.

Previous animal models for studying arrhythmias frequently used (Hynes et al., 2002) aorta bandage to create ischemic condition (Billman, 2002; 2005), (2) fast pacing to induce heart failure (Shinbane et al., 1997; Moe et al., 1998; Kashem et al., 2003; Timek et al., 2003), (3) genetic ablation (Benkusky et al., 2007). Significant progress has been made regarding the underlying mechanisms of a variety of cardiac arrhythmias. All these studies have led to the ion channel remodeling under each condition. These studies created either a structural change in heart (ischemia, heart failure) or an altered expression level of a specific protein. However, it remains as a puzzle as to how heart stop beating without structural changes and unaltered expression levels of relevant proteins. In fact, an ion channel protein has multiple functions. For example, the selectivity of a channel is controlled by the amino acids lining in the pore regions between S5 and S6 domains. The activation, inactivation and deactivation of a channel can be controlled by amino acids in the $\mathrm{N}$ - or C-terminal regions (Staes et al., 2001; Gustina et al., 2011; Takahiro et al., 2007). In addition, the gating kinetics of a channel can be controlled by amino acids in other transmembrane domains (Hanlon et al., 2002). 
These biophysical studies have led to the recent discoveries of many single amino acid mutations of channels identified in patients with arrhythmias such as long QT (Moss et al., 2005; Hedley et al., 2009).

To explore new mechanisms independent of cardiac structural changes, seen in ischemia and heart failure, or mutations in ion channels, we established an inducible rat arrhythmia model to investigate how altered state of tyrosine phosphorylation might be relevant to the arrhythmogenesis.

Tyrosine phosphorylation has recently been demonstrated to be involved in the regulation of gating properties and surface expressions of pacemaker channels, which may in turn result in the alteration of cardiac tune (Huang et al., 2008; Lin et al., 2009). Inhibition of tyrosine phosphatases may associate with the enhancement of tyrosine kinase-mediated tyrosine phosphorylation and thus change the ventricular activity.

Our results showed that sodium orthovanadate, a tyrosine phosphatase inhibitor (Gordon, 1991), can reliably induce several types of ventricular arrhythmias at relatively low doses. These results indicated our initial success in the establishment of a valid in vivo rat model for studying the novel mechanisms (such as tyrosine phosphorylation) of ventricular arrhythmias in the absence of cardiac structural remodeling and channel mutations. 


\section{Results}

In this study, two different doses of sodium orthovanadate were administrated via jugular vein injection. In order to investigate whether ventricular arrhythmias can be induced, I applied the dose of sodium orthovanadate at $3.8 \mathrm{mg} / \mathrm{kg}$. PVC, fragmented QRS (Fig 2), and ventricular tachycardia (VT) (Fig 3) can be induced by this dose in comparison with the basal (Fig 1). It is worth to notice here that Torsades de pointes, a rapid, polymorphic ventricular tachycardia, followed by frequent VT can also be recorded at the dose of 3.8 $\mathrm{mg} / \mathrm{kg}$ (Fig 3).

For the more serious ventricular arrhythmias induction, an increasing dose up to 5.5 $\mathrm{mg} / \mathrm{kg}$ sodium orthovanadate was applied. The reliable induction of ventricular fibrillation can be recorded by this dose (Fig 4).

These results suggested that different types of ventricular arrhythmias can be reliably induced depending upon different doses of sodium orthovanadate administration. Thus, this established animal model can be served as a good model to study ventricular arrhythmias mediated by the alteration of tyrosine phosphorylation state. 


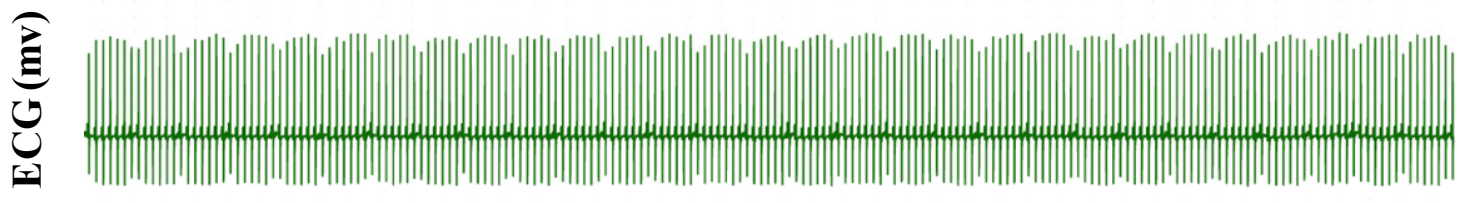

\section{主 \\ ?ִ}
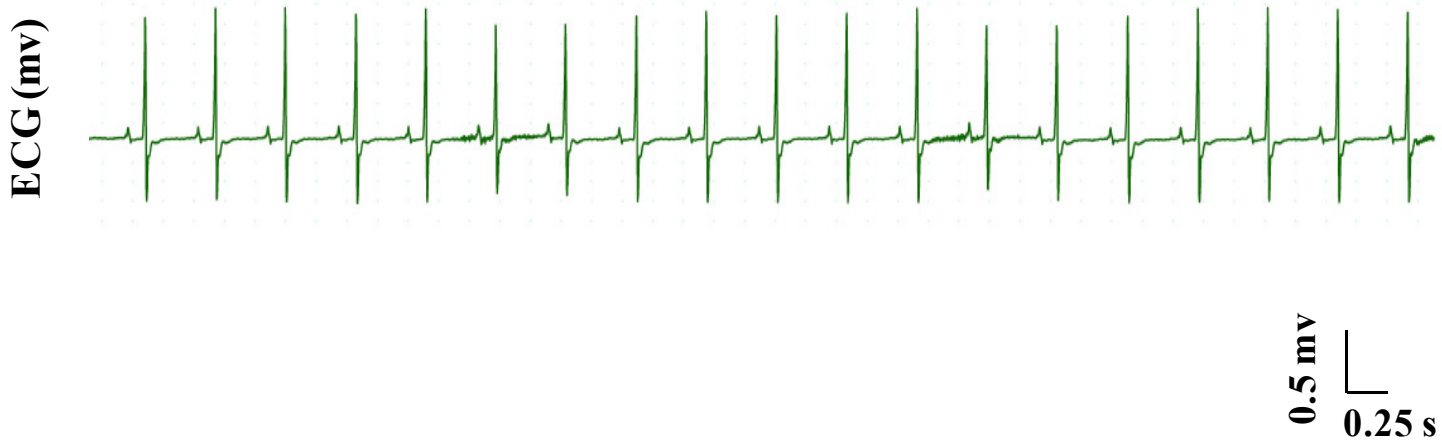

Fig 1. Normal ECG in anesthetized SD rats.

Upper panel indicates the overview of the measurement of ECG. Lower panel indicates the enlarged scope from the upper panel. X-axis represents the time scale (sec), Y-axis represents the amplitude (mv) of ECG. 

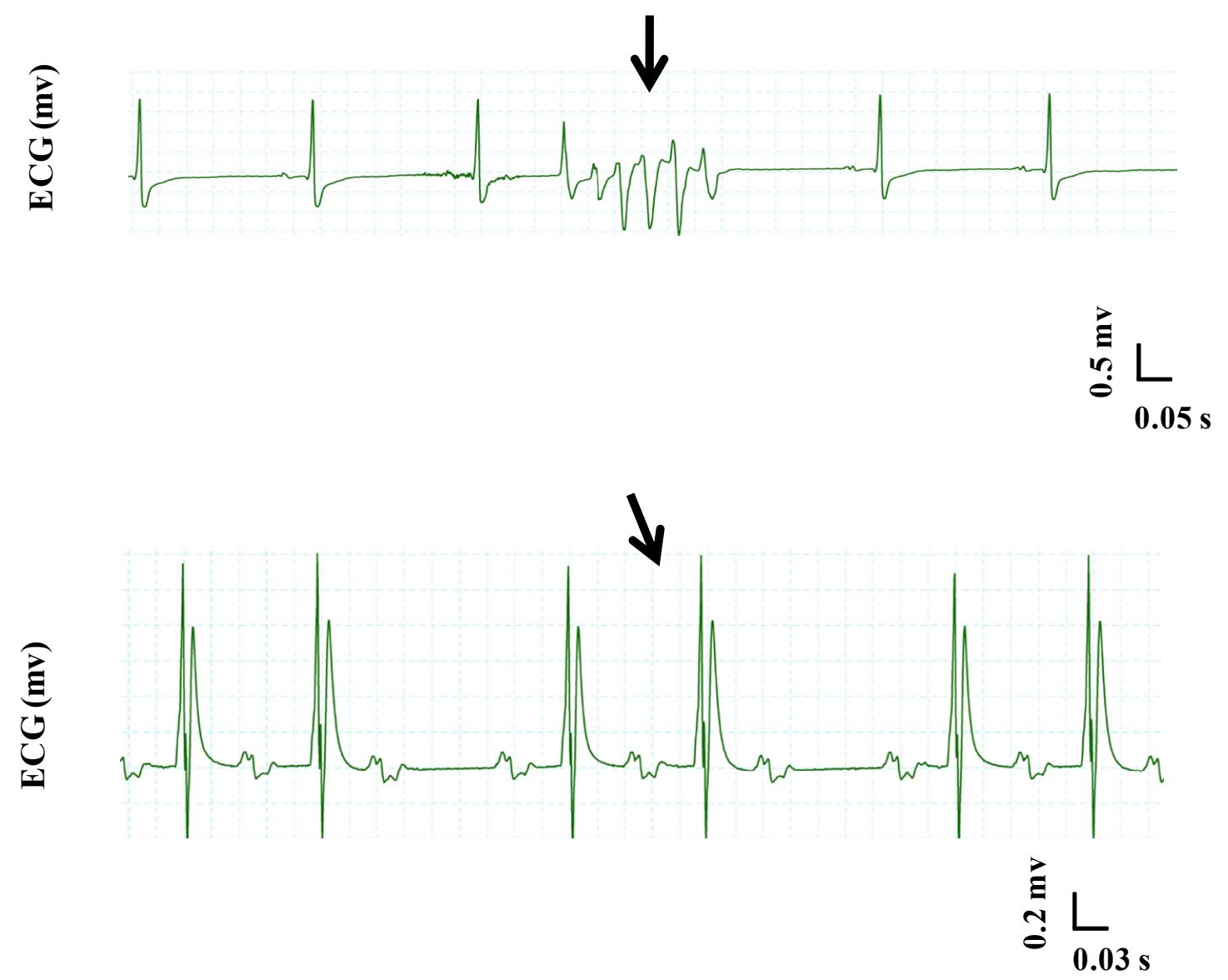

Fig 2. PVC and fragmented QRS were induced by $\mathrm{Na}_{3} \mathrm{VO}_{4}$ at the dose of $3.8 \mathrm{mg} / \mathrm{kg}$.

Upper panel represents multiple PVCs (black arrow) whereas lower panel indicates fragmented QRS (black arrow) which were induced in a dose of $3.8 \mathrm{mg} / \mathrm{kg}$. 


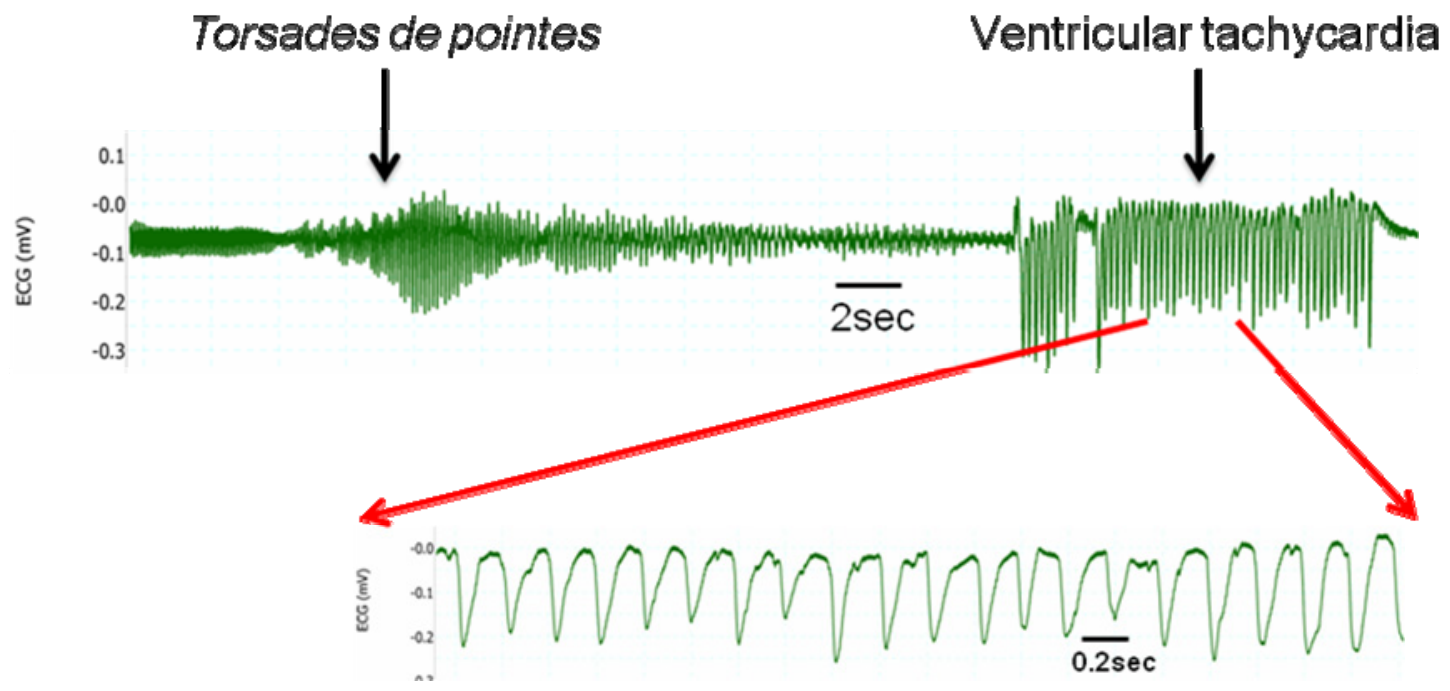

Fig 3. Ventricular tachycardia (VT) was induced by $\mathrm{Na}_{3} \mathrm{VO}_{4}$ at the dose of 3.8 mg/kg.

Torsades de pointes, a rapid, polymorphic ventricular tachycardia, followed by frequent VT (black arrow in upper panel) can also be recorded by applying $\mathrm{Na}_{3} \mathrm{VO}_{4}$ at the dose of $3.8 \mathrm{mg} / \mathrm{kg}$. Red line enclosed traces indicates the enlarged scope of VT from the upper panel. 

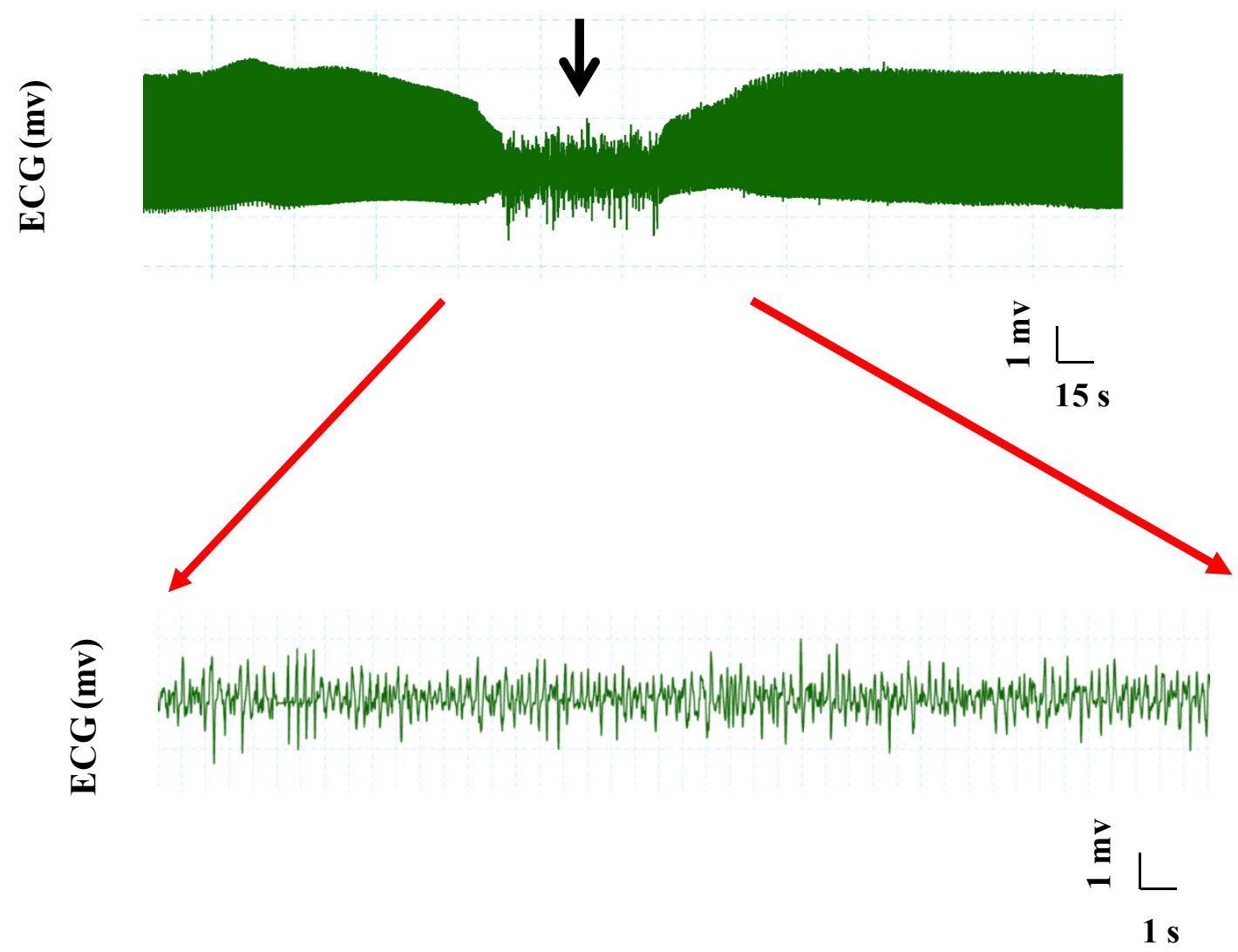

Fig 4. Ventricular Fibrillation (VF) was induced by $\mathrm{Na}_{3} \mathrm{VO}_{4}$ at the dose of 5.5 $\mathbf{m g} / \mathbf{k g}$. Upper panel indicates the overview of VF (black arrow) whereas red line enclosed traces represents the enlarged scope from the upper panel. 


\section{Discussion}

Several cardiac diseases such as ischemia, hypertrophy, and cardiac sudden death are associated with ventricular arrhythmias. However, the underlying mechanism of how ventricular arrhythmias occur is still obscure due to lack of a feasible animal model with the controlled ventricular arrhythmias. By using sodium orthovanadate, a tyrosine phosphatase inhibitor, we established a valid small-molecule inducible rat model to study the mechanisms of ventricular arrhythmias without cardiac structural remodeling.

Sodium orthovanadate is a small molecule which can rapidly enter the cell to inhibit cellular tyrosine phosphatase activity. Our previous studies demonstrated that tyrosine phosphorylation was involved in the regulation of surface expression and gating properties of $\mathrm{HCN}$ channels, which may consequently lead to the alteration of ventricular activities (Huang et al., 2008; Lin et al., 2009). Thus, reduced tyrosine phosphatase activity may be involved in tyrosine kinase activity increasing and tachycardia-mediated ventricular arrhythmias. Surprisingly, by using two different doses of sodium orthovanadate, our results demonstrated that different dose-based types of ventricular arrhythmias can be reliably induced, possibly through increasing HCN channel activities (either increase surface expressions or gating properties) mediated by increased tyrosine phosphorylation.

This model will help us understand how tyrosine phosphorylation contributes to ventricular arrhythmogenesis and is useful in effort to test small molecules to delay and ultimately inhibit the incidents of ventricular arrhythmias which in most situations are lethal. 


\title{
Part II: Inhibition of Src kinase activity reduces heart rate without significant alteration of heart rate variability
}

\begin{abstract}
Heart rate variability (HRV) is the cardiac index representing the oscillation of the intervals between consecutive heartbeats (RR intervals in the ECG). It reflects the influences of the autonomic nervous system (ANS) on the sinus node. In order to investigate the involvement of tyrosine phosphorylation in cardiac function of the whole rat system and its effect on HRV, we employed a potent selective inhibitor of Src family kinases, PP2 (4-amino-5-(4-chlorophenyl)-7-(t-butyl)pyrazolo[3,4- $d]$ pyramidine), to inhibit the activity of Src kinases that include Src, Fyn, and Yes.
\end{abstract}

Heart rate was decreased $20 \%$ by PP2 at the dose of $0.2 \mathrm{mg} / \mathrm{kg}$. PP3, a negative control compound of PP2, which does not inhibit the activity of Src kinases decreased heart rate by $8 \%$ (when at the identical dose of $0.2 \mathrm{mg} / \mathrm{kg}$ ) when compared with the basal. Interestingly, HRV was not significantly altered at a relatively low dose of PP2 (0.2 $\mathrm{mg} / \mathrm{kg}$ ) when compared with that of PP3 or the basal. Unlike that of PP2, HRV was significantly decreased after application of $0.4 \mu \mathrm{g} / \mathrm{kg}$ isoproterenol. Similar to that of PP2, ZD7288, a specific inhibitor of HCN channels, when at the low dose of $0.2 \mathrm{mg} / \mathrm{kg}$, was able to decrease the heart rate without significant change of HRV in comparison to isoproterenol. 
Furthermore, atrial ventricular block (AV block) can be induced at the higher dose of PP2 $(0.3 \mathrm{mg} / \mathrm{kg})$ whereas in ZD7288, higher dose application $(0.7 \mathrm{mg} / \mathrm{kg})$ can elicit both premature ventricular contraction (PVC) and AV block.

Although PP2 and ZD7288 slightly increased the short-term (SD1) and the long-term (SD2) HRV, larger degree of increase in SD1 or SD2 was observed in isoproterenol. The fractal scaling exponent alpha $1(\alpha 1)$ in basal level was 0.640 , which was comparable to that of PP3 (0.605), but slightly decreased in PP2 (0.328) and ZD7288 (0.144) at the low dose of $0.2 \mathrm{mg} / \mathrm{kg}$.

Overall, PP2, like ZD7288, can serve as a potential candidate in the treatment of tachycardia-induced arrhythmias via inhibition of Src-mediated tyrosine phosphorylation. 


\section{Introduction}

Sudden cardiac death caused by cardiac arrhythmias has affected nearly 300,000 people in the United States every year and contributes to approximately $50 \%$ of all death of cardiovascular causes (Myerburgh et al., 1997). Tachycardia, represented fast heart beat, has been demonstrated to be the common reason to cause cardiac arrhythmias (Kannel et al., 1987; Willich et al., 1987). However, the underlying mechanism of cardiac arrhythmias is still obscure. In the previous study by using tyrosine phosphatase inhibitor sodium orthovanadate in a whole animal rat model, we demonstrate that different degree of ventricular arrhythmias can be reliably induced by decreasing endogenous tyrosine phosphastase activity. Here we demonstrated that reduction of Src-mediated tyrosine phosphorylation by its specific inhibitor PP2 decreased the heart rate to nearly $20 \%$ at low dose of $0.2 \mathrm{mg} / \mathrm{kg}$ in comparison to the basal.

HRV is the cardiac index representing oscillation of RR interval of ECG, reflecting the effect of autonomic system on the sinus node. Accumulating evidence indicates that there is a strong association between the compromised autonomic nervous system and sudden cardiac death (Jouven et al., 2005; Schwartz et al., 1992; 1998). HRV has been widely used to measure alterations in the autonomic nervous system, which in turn influences the rhythm of heart. Several studies have shown that decreased HRV can serve as a powerful predictor for sudden cardiac death than established clinical predictors such as left ventricular ejection fraction (Bauer et al., 2006; Makikallio et al., 2005; Scmiddt et al., 1999). Decreased HRV has also been shown to be directly link to the risk of long-term mortality (Laitio et al., 2000; 2002; 2004; Stein et al., 2001; Wu et al., 2005). Thus, a 
treatment of sudden cardiac death by strategically decrease tachycardia-induced arrhythmias with little change of HRV is especially important for the sudden cardiac death.

Here, we found that the inhibition of Src-mediated tyrosine phosphorylation by its specific inhibitor PP2 can significantly decrease heart rate at a relatively low dose without a significant alteration of HRV (in comparison with the effect of isoproterenol on HRV). Thus, PP2, like ZD7288, may serve as a potential candidate in the treatment of tachycardia-induced arrhythmias via inhibition of Src-mediated tyrosine phosphorylation when at relatively low dosage. 


\section{Results}

\section{Representative traces of ECG in the administration of different drugs}

In Fig 1, high dose of PP2 (0.3 mg/kg) or ZD7288 $(0.7 \mathrm{mg} / \mathrm{kg})$ (lower panel) as well as low dose of PP2 or ZD7288 (0.2 $\mathrm{mg} / \mathrm{kg})$ (upper panel) were applied and exhibit the waveforms of ECG. At the high dose of PP2, multiple AV block can be induced. PVC (dash arrow) or AV block (black line) can also be elicited at high dose of ZD 7288 (lower panel). The identical low dose $(0.2 \mathrm{mg} / \mathrm{kg})$ of PP3 was applied as a negative control of PP2. The dose of $0.4 \mu \mathrm{g} / \mathrm{kg}$ isoproterenol was used as the positive control of PP2 and ZD7288 effects on heart rate and HRV.

\section{The effects of different drugs on HRV}

In Fig 2, the distribution pattern of HRV reveals bell shape distribution in the basal (the first left and middle panel). HRV was slightly decreased by PP2 (the fourth panel) in comparison to isoproterenol which significantly decreased HRV (the second panel). PP3 or ZD7288 reduces HRV very slightly in comparison to the basal. We also compared the distribution pattern of heart rate and HRV. Interestingly, the distribution pattern of heart rate in each drug is comparable (right panels) to that of HRV.

\section{HRV analysis in Poincare Plot}

Poincare plot is a geometric method normally used for dynamic analysis of HRV in which the correlation between each RR interval and the preceding RR interval can be plotted, indicating the degree of RR interval complexity. As indicated in Fig 3, Poincare plot analysis includes the parameters of short-term HRV (SD1) and long-term HRV (SD2), which slightly increase in PP2 (SD1=10.0, SD2=7.1) and ZD7288 (SD1=5.6, 
$\mathrm{SD} 2=5.9)$, but significantly increased in isoproterenol $(\mathrm{SD} 1=30.9, \mathrm{SD} 2=30.3)$ in comparison to the basal $(\mathrm{SD} 1=1.1, \mathrm{SD} 2=1.2)$. The values of $\mathrm{SD} 1$ and $\mathrm{SD} 2$ in PP3 $(\mathrm{SD} 1=1.8, \mathrm{SD} 2=1.6)$ were more comparable to those in the basal, indicating that PP3 has the similar beat-to-beat variability with the basal.

The fractal scaling exponent alpha $1(\alpha 1)$ is the slope of the plot between logarithm of the RR interval fluctuation and the logarithm of the size of time scale indicating selfsimilarity of heart rate dynamics, representing how random of heart rate dynamics is. In PP3, the value of $\alpha 1$ was 0.605 , which is comparable to that of basal level (0.640). The values of $\alpha 1$ were slightly decreased in PP2 (0.328) and ZD7288 (0.144) at a relative low dose of $0.2 \mathrm{mg} / \mathrm{kg}$ in comparison to the basal and isoproterenol ( $\alpha 1$ slightly increase $(0.742))$.

\section{HRV results in the presence of different drugs administration}

Table 1 is the summary of the representative values of different parameters for each drug. We applied $0.2 \mathrm{mg} / \mathrm{kg}$ in PP2, PP3, and ZD 7288, and $0.4 \mu \mathrm{g} / \mathrm{kg}$ in isoproterenol. Each experiment was repeated at least ten times $(\mathrm{n}=10-12)$. From this table, heart rate significantly decreased $20 \%$ in PP2 and 35\% in ZD7288 when compared with the basal. PP3 can also decrease heart rate by $8 \%$. The percentage decrease of heart rate is identical to the percentage increase of mean RR. The longer the RR interval is, the slower the heart rate will be. Standard deviation of R-R interval (STD RR) revealed slight increasing in PP2 (8.7) and ZD7288 (5.7) whereas significantly increase in isoproterenol (30.6) in comparison with PP3 (1.8) and the basal (1.1). SD1, SD2, and $\alpha 1$ have been analyzed in Fig 3 where there is a slight change in PP2. 
Overall, our data strongly demonstrate that PP2 is able to reduce heart rate without a significant alteration of HRV (in comparison to the effect of isoproterenol on HRV). 


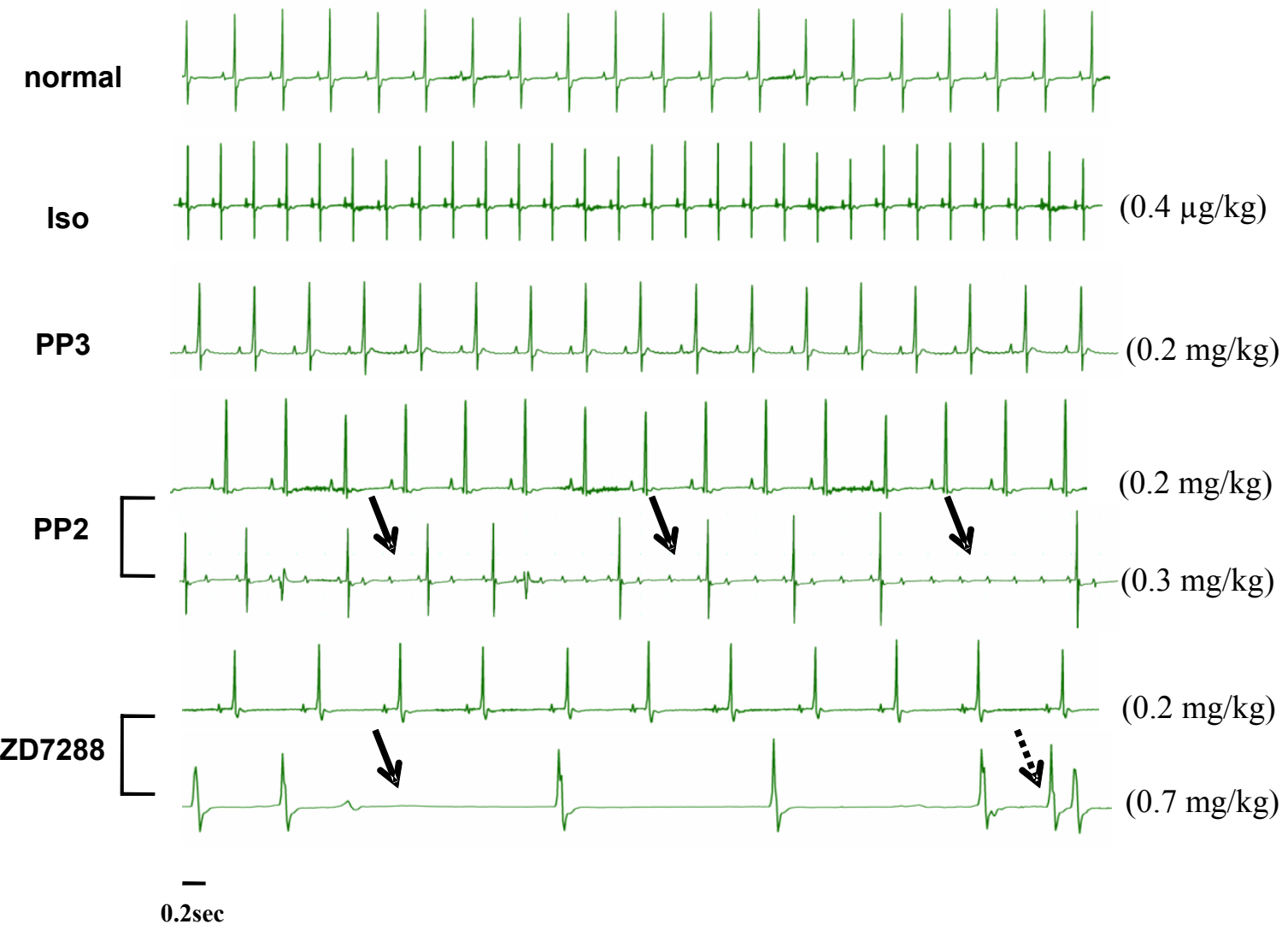

Fig 1. ECG in PP2, ZD7288, and controls.

Representative waveforms of ECG can be recorded in the presence of different drugs. High dose of PP2 $(0.3 \mathrm{mg} / \mathrm{kg})$ or ZD7288 $(0.7 \mathrm{mg} / \mathrm{kg})$ as well as low dose of PP2 or ZD7288 $(0.2 \mathrm{mg} / \mathrm{kg})$ were applied and the waveforms of ECG were exhibited. In PP2, the upper panel represents the ECG in low dose application whereas in high dose application in the lower panel. Multiple AV (black arrow) block can be induced at high dose application of PP2. In ZD7288, high dose application (lower panel) can also elicit PVC (dash arrow) or AV block (black arrow). PP3, a negative control of PP2, was applied at the dose of $0.2 \mathrm{mg} / \mathrm{kg}$ for the comparison with low dose of PP2. $0.4 \mu \mathrm{g} / \mathrm{kg}$ isoproterenol was applied for the positive control of PP2 and ZD7288. The ECG of the basal was also shown in the first panel as the control $(\mathrm{n}=10)$. 

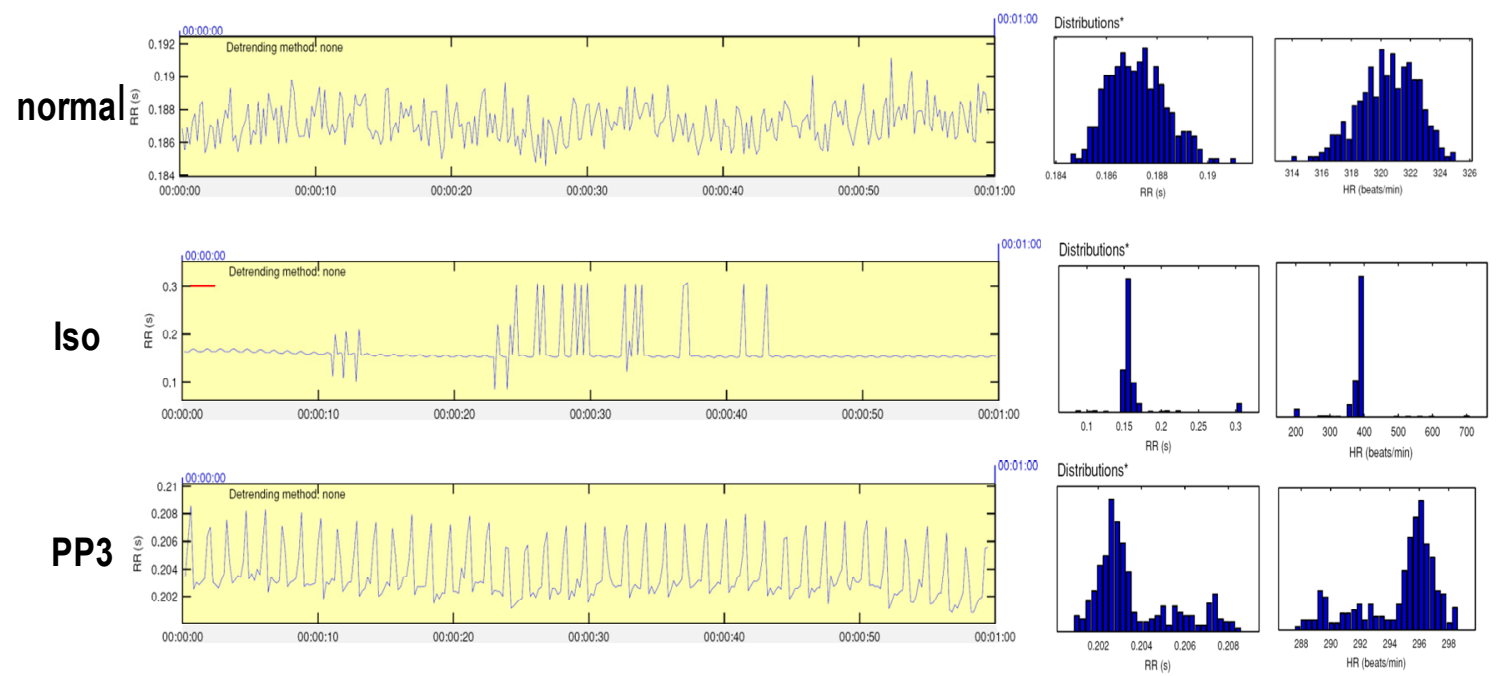

PP2
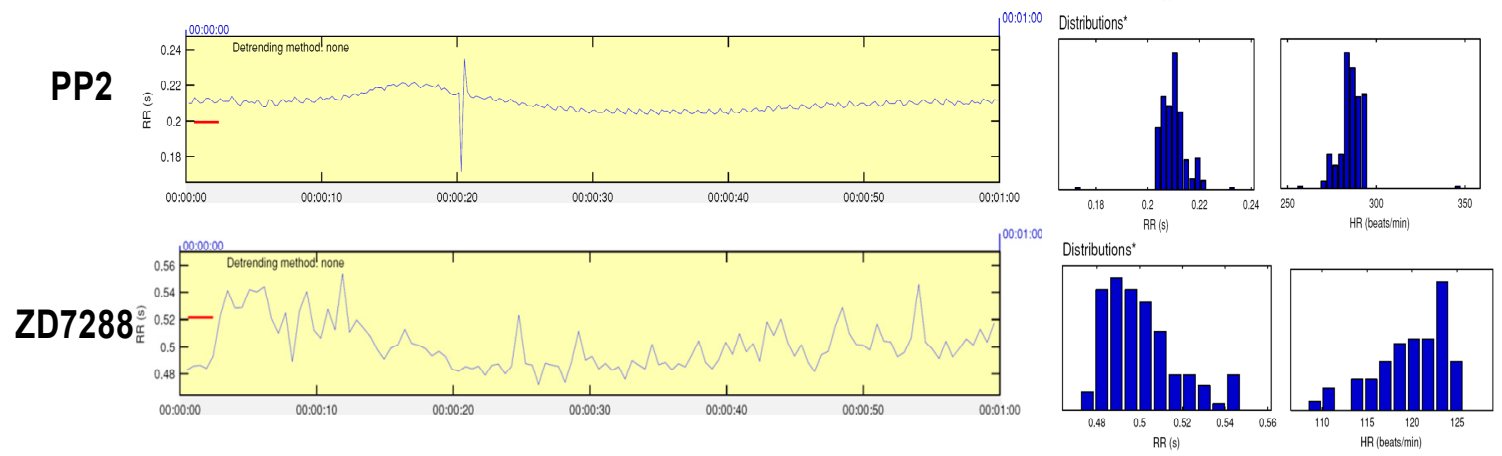

Fig 2. HRV in PP2, ZD7288, and controls.

HRV reveals bell shape distribution pattern in the basal (the first left and middle panel). Isoproterenol (the second panel) reveals significant whereas PP2 (the fourth panel) indicates slight reduction of HRV. PP3 or ZD7288 reduces very slightly in HRV in comparison with the basal. The distribution pattern of HR in each drug is in an inverse trend (right panels) with that of HRV since HR is inversely proportional to HRV. The figure shown above is the representative HRV for each drug $(\mathrm{n}=10-12)$. 


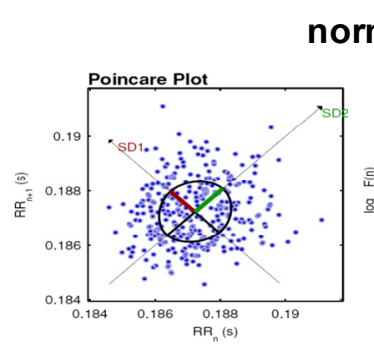

\section{normal}
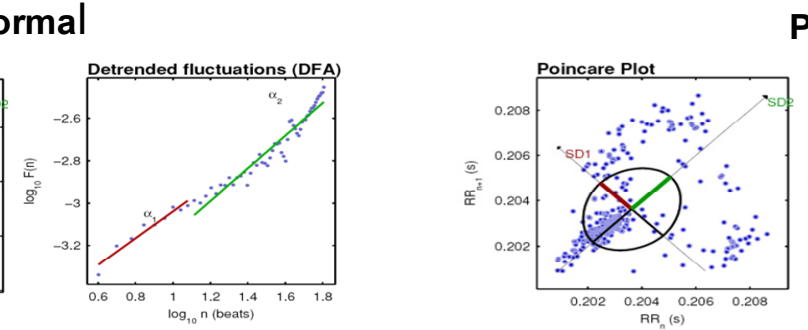

PP3

Iso
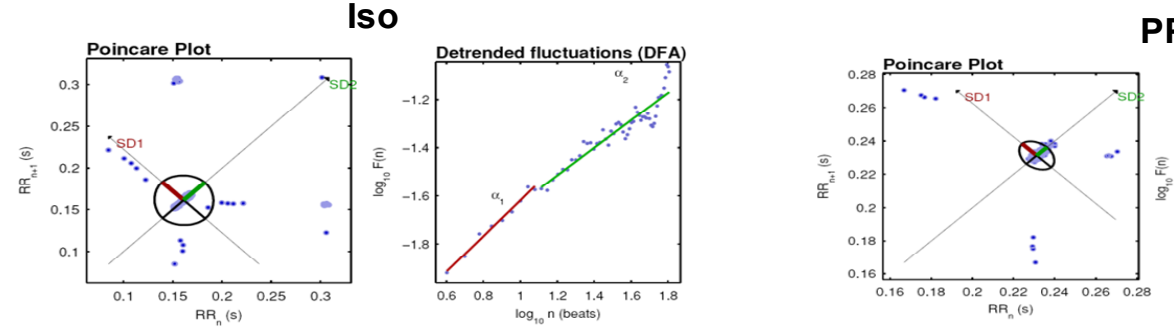

PP2
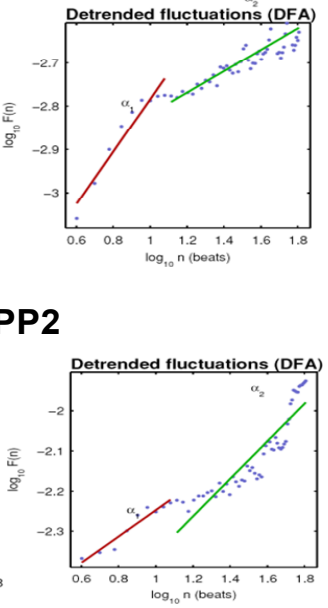

\section{ZD7288}

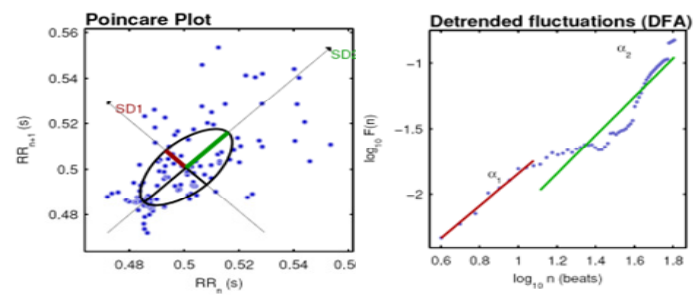

\section{Fig 3. HRV analysis in Poincare Plot.}

Poincare plot analysis also revealed that both of short-term HRV (SD1) and long-term HRV (SD2) were slightly increased in PP2 and ZD7288, but significantly increased in isoproterenol in comparison with the basal. The fractal scaling exponent alpha $1(\alpha 1)$ in basal level is 0.640 , which is comparable with that of PP3 (0.605), but decrease in PP2 $(0.328)$ and ZD7288 (0.144) at the low dose of $0.2 \mathrm{mg} / \mathrm{kg}$. 


\begin{tabular}{|c|c|c|c|c|c|c|}
\hline Drugs/Variable & Mean HR (bmp) & Mean RR (ms) & STD RR (ms) & SD1 (ms) & SD2 (ms) & $\alpha 1$ \\
\hline Basal & 320 & 187.2 & 1.1 & 1.1 & 1.2 & 0.640 \\
\hline Iso & 380 & 161.2 & 30.6 & 30.9 & 30.3 & 0.742 \\
\hline PP3 & 294 & 203.6 & 1.8 & 1.6 & 2.0 & 0.605 \\
\hline PP2 & 259 & 231.3 & 8.7 & 10.0 & 7.1 & 0.328 \\
\hline ZD7288 & 205 & 291.5 & 5.7 & 5.6 & 5.9 & 0.144 \\
\hline
\end{tabular}

Table 1. HRV results with drugs.

The representative values of different parameters for each drug were calculated from the kubios software. The applied dose of PP2, PP3 and ZD7288 was at $0.2 \mathrm{mg} / \mathrm{kg}$ whereas isoproterenol was at $0.4 \mu \mathrm{g} / \mathrm{kg}$. Each experiment was repeated at least ten times $(\mathrm{n}=10-$ 12). The values shown above were selected from the representative data. 


\section{Discussion}

Heart rate variability includes time domain and frequency domain which is normally used to depict the effects of autonomic nervous system on the sinus node. Time domain includes the parameters of RR interval, standard deviation of RR interval (STD RR). Frequency domain includes the parameters of short-term HRV (SD1) and long-term HRV (SD2) in Poincare plot as well as the fractal scaling exponent alpha $1(\alpha 1)$ (Goldberger et al., 2002; Peng et al., 1995; Pumprla et al., 2002; RajendraAcharya et at., 2006; Thuraisingham et al., 2006).

Poincare plot is a geometric method normally used for dynamic analysis of HRV in which the correlation between each RR interval and the preceding RR interval is plotted. It represents the degree of RR interval complexity. There are two parameters used for Poincare plot analysis: short-term HRV (SD1) and long-term HRV (SD2). SD1 represents the points vertical to the line of identity and reflects beat-to-beat variability. SD2 represents the dispersion of the RR interval points along the line of identity and reflects the long-term records of HRV (Brunetto et al., 2005; Smith et al., 2007). Our data reveal slight increase of SD1 and SD2 in PP2 and ZD7288 indicating PP2 and ZD7288 will not significantly change the RR interval.

$\alpha 1$ is the slope of the plot between logarithm of the RR interval fluctuation and the logarithm of the size of time scale, representing self-similarity of HR dynamics: how random of the relationship between logarithm of the RR interval fluctuation and the logarithm of the size of time scale. When compared with the control, the higher or lower number indicates the random of RR interval dynamics (which means in less fractal 
pattern) (Peng et al., 1993; Weibel et al., 1991). The slight decrease of $\alpha 1$ in PP2 and ZD7288 when compared with PP3 and the basal represented that these two drugs will not significantly cause the loss of fractal correlation properties of HR dynamics toward more random HR dynamics.

ZD7288, a selective blocker of HCN channel, has become a potent drug clinically used for the treatment of tachycardia in Europe. Here, we test the effect of ZD7288 on HRV as the comparison with PP2. Our results indicate that at the low dose $(0.2 \mathrm{mg} / \mathrm{kg})$ of ZD7288 and PP2, heart rate was significantly reduced nearly $35 \%$ and $20 \%$, respectively, when compared with the basal (which inversely corresponds to the percentage change of mean RR interval) with slight change of HRV. However, PP3, a negative control compound of PP2, which does not inhibit the activity of Src kinases, can decrease heart rate by $8 \%$. Whether PP3 involves in signal cascades that contribute to the regulation of heart rate is still unknown and need to be investigated. A $\beta$ adrenergic receptor agonist, isoproterenol, is also applied here as a positive control showing the increase of the heart rate. Especially, the distribution pattern of the RR interval in ZD7288 and PP2 has a slight change in comparison to that of the basal or PP3.

Our previous studies demonstrated that Src-mediated tyrosine phosphorylation plays a pivotal role in the regulation of activities of HCN channels in HEK 293 cells. Reduced Src-mediated tyrosine phosphorylation results in deceased activities of HCN channels including reduction of surface expression and gating properties of the channels. PP2, a specific Src kinase inhibitor, can reduce surface expression and gating properties of $\mathrm{HCN}$ channels via decrease Src-mediated tyrosine phosphorylation in HEK 293 cells. In order 
to see whether PP2 can affect the heart rate through decrease of HCN channel activities, we employ a whole animal rat in this study to investigate the potential role on the heart rate regulation. Indeed, we found that the heart rate was significantly reduced after applying PP2 within the time scale of minutes at a relative low dose.

The different percentage decrease of heart rate in ZD7288 and PP2 may be due to intrinsically mechanistic difference. ZD7288 inhibits HCN channels by penetrating into the cells and directly blocks the pore region of the channels. As HCN channels are the pacemaker channels associated with the heart rate, inhibition of the channels by ZD7288 results in more efficient reduction of the heart rate than PP2. Src-mediated tyrosine phosphorylation regulates gating properties of other ion channels and exchangers, which can contribute to an increase in heart rate.

The association between Src and adrenergic receptors has been reported in the previous study (Huang et al., 2004; Naohiro et al., 2007). The slight effect of PP2 on HRV may be attributed to disruption of this association where the inhibition of Src kinases by PP2 may somehow cause the change of adrenergic receptor-mediated signal cascades and slightly influence HRV. Further studies are needed for the confirmation.

Atenolol (used to substitute the non-specific propranolol) and Metoprolol are the $\beta 1$ specific inhibitors used for the treatment of cardiac arrhythmias. However, side effects caused by them implicate the potential problems. Through significant reduction of heart rate by decreased Src-mediated tyrosine phosphorylation, our studies strongly indicate that PP2 may serve as a potential candidate for the treatment of tachycardia-induced arrhythmias without significant alteration of HRV. 


\section{Chapter 2}

\section{Elucidate cellular mechanisms of tyrosine phosphorylation in}

\section{the regulation of heart rate and cardiac arrhythmias}

The goal of this chapter is to elucidate the cellular mechanism of tyrosine phosphorylation in the regulation of cardiac pacemaker channels that directly contribute to the regulation of heart rate and their potential new role in cardiac arrhythmias. Two parts are included. Part I summarizes the role of tyrosine phosphorylation in rescuing a trafficking defective pacemaker channel mutant. Part II summarizes the role of tyrosine phosphorylation in the modulation of pacemaker channels. The results in this chapter have been published (J Biol Chem 284: 30433-30440, 2009). 


\title{
Part I: Rescue of a Trafficking Defective Human Pacemaker Channel via a Novel Mechanism: Role of Src, Fyn, and Yes Tyrosine Kinases
}

\begin{abstract}
Therapeutic strategies such as using channel blockers and reducing culture temperature have been used to rescue some long QT-associated voltage-gated potassium Kv trafficking defective mutant channels. A hyperpolarization-activated cyclic nucleotidegated HCN4 pacemaker channel mutant (D553N) has been recently found in a patient associated with cardiac arrhythmias including long QT. D553N showed the defective trafficking to the cell surface, leading to little ionic current expression (loss-of-function). We show in this report that enhanced tyrosine phosphorylation mediated by Src, Fyn, and Yes kinases was able to restore the surface expression of D553N for normal current expression. Src or Yes, but not Fyn, significantly increased the current density and surface expression of D553N. Fyn accelerated the activation kinetics of the rescued D553N. Co-expression of D553N with Yes exhibited the slowest activation kinetics of D553N. A combination of Src, Fyn, and Yes rescued the current expression and the gating of D553N comparable with those of wild-type HCN4. In conclusion, we demonstrate a novel mechanism using three endogenous Src kinases to rescue a trafficking defective HCN4 mutant channel (D553N) by enhancing the tyrosine phosphorylation of the mutant channel protein.
\end{abstract}




\section{Introduction}

Defective trafficking leading to the reduced surface expression of ion channels is one of the mechanisms responsible for a loss-of-function of the ion channel on the plasma membrane (Delisle et al., 2004). Several methods have been developed to rescue the voltage-gated potassium $\mathrm{Kv}$ trafficking defective channels: reducing the culture temperature, applying the channel blockers, altering the molar ratio of glycerol, and using the sarcoplasmic/endoplasmic reticulum $\mathrm{Ca}^{2+}$-ATPase inhibitor thapsigargin (Delisle et al., 2003; Furutani et al., 1999; Paulussen et al., 2002; Zhou et al., 1998; 1999;).

Hyperpolarizing-activated cyclic nucleotide-gated $(\mathrm{HCN})$ pacemaker channels generate time- and voltage-dependent inward currents, named $I_{\mathrm{h}}$ in neurons or $I_{\mathrm{f}}$ in the heart (Robinson et al., 2003). They are important in various cell functions including excitability, synapse transmission, and rhythmic activity (Robinson et al., 2003). The most well studied regulation of $I_{\mathrm{f}}$ is its response to autonomic stimulation. $\beta$-Adrenergic receptor activation increases and acetylcholine receptor activation decreases the intracellular cAMP levels, which in turn increases/decreases $I_{\mathrm{f}}$ by binding to the cyclic nucleotide-binding domain of the HCN channels, respectively (Robinson et al., 2003). Other important mechanisms for the modulation of $I_{\mathrm{f}} / \mathrm{HCN}$ channels have recently been found including $\beta$-subunit (Yu et al., 2001), lipids (Fogle et al., 2007; Pian et al., 2006), and p38 mitogen-activated protein kinase (Poolos et al., 2006).

Accumulating evidence has revealed tyrosine phosphorylation as an important mechanism for modulation of HCN channel properties (Arinsburg et al., 2006; Li et al., 2008; Wu et al., 2000; Yu et al., 2006; Zong et al., 2005). An acute increase in tyrosine 
phosphorylation of $I_{\mathrm{f}}$ or $\mathrm{HCN}$ channels increases the channel activity, including an increase in the current amplitude, a positive shift of the voltage-dependent activation, an acceleration of activation kinetics, and an increase in whole cell conductance (Arinsburg et al., 2006; Li et al., 2008; Wu et al., 2000; Yu et al., 2006). Recently, we discovered that the cell surface expression of HCN2 channels can be remarkably inhibited by tyrosine dephosphorylation mediated by receptor-like protein tyrosine phosphatase $\alpha$ $(\mathrm{RPTP} \alpha)$ and increased by tyrosine phosphorylation via Src kinase after long term treatment (Huang et al., 2008).

D553N, a missense HCN4 mutant, was recently identified in a patient with cardiac arrhythmia associated with depressed HCN gating properties (Ueda et al., 2004). Functional and structural assays revealed that D553N expresses little ionic currents, which is possibly due to the defective channel trafficking so that the channels cannot reach the plasma membrane for normal functions (Ueda et al., 2004).

The Src kinase family has nine members (Thomas et al., 1997). They are closely related and share the same regulatory function. Three of them, Src, Fyn, and Yes, are ubiquitously expressed in a variety of tissues including neurons and myocytes (Kalia et al., 2004; Thomas et al., 1997). Without stimulation, they are inactive. However, mutation of key tyrosine residue results in the constitutively active form of the kinase, SrcY529F, FynY531F, and YesY537F, respectively (Barraclough et al., 2007; Kanda et al., 2005). Using these Src kinases, we show in this report a novel approach that can restore the surface expression of D553N for normal current expression via tyrosine phosphorylation. 


\section{Results}

\section{Inhibition of HCN4 Current Expression by RPTPa}

We have recently demonstrated that RPTP $\alpha$ can inhibit the surface expression of HCN2 channels via tyrosine dephosphorylation (Huang et al., 2008). Given the high structural homology between HCN2 and HCN4 (>80\%) (Robinson et al., 2003), it was expected that RPTP $\alpha$ may also inhibit the surface expression of HCN4. Fig. 1 shows a typical current expression of HCN4 expressed in HEK293 cells (Fig. 1A). The expression was dramatically suppressed across the test voltages $(-65$ to $-135 \mathrm{mV})$ when HCN4 was coexpressed with RPTP $\alpha$ after 1 day of transfection (Fig. 1B). The reduction in current expression was associated with a negative shift in threshold activation (Figs. 1, $A$ and $B$, arrows). After 2 days of transfection, RPTP $\alpha$ almost eliminated the current expression of HCN4 (Fig. 1C). The effect of RPTP $\alpha$ on HCN4 current expression is similar to that on HCN2 current expression (Huang et al., 2008). As a control, the empty vector, pRK5 (used to subclone RPTP $\alpha$ ), did not affect the current expression of HCN4 (Fig. 1D). Each of these results was confirmed in additional 5-7 cells.

\section{Inhibited Surface Expression of HCN4 by RPTPa}

To further seek supporting evidence that reduced ionic current expression of HCN4 is caused by the suppressed surface expression of HCN4 channels, we tagged HCN4 with a fluorescent protein, DsRed, and examined the distribution of HCN4 using fluorescent confocal microscopy. Fig. $2 A$ shows a typical fluorescent image of HCN4 expressed alone in a HEK293 cell (left panel). When co-expressed with RPTP $\alpha$, most HCN4 channels are retained in the cytosol (middle panel of Fig. $2 A$ ). On the other hand, Src529 
significantly enhanced the cell surface expression of HCN4 (Fig. 2A, right panel). As a control, Fig. $2 B$ shows the fluorescence (left panel) and bright field (right panel) images of the empty DsRed vector expressed in HEK293 cells.

\section{Rescuing D553N Current Expression by Src, Fyn, and Yes}

D553N has been recently identified in a patient suffering from sinus node dysfunction, long QT, ventricular tachycardia, and torsade de pointes (Ueda et al., 2004). In vitro studies of the mutant channel revealed defective surface expression on plasma membrane, leading to the loss of current expression (Ueda et al., 2004). Given the facts that the HCN4 channel activity including the channel surface expression can be significantly enhanced by Src-mediated tyrosine phosphorylation and the ubiquitous expression of three Src kinase family members (Src, Fyn, and Yes), we set forth to test the hypothesis that the current expression of the defective trafficking D553N can be restored by constitutively active forms of Src kinases (Src529, Fyn531, and Yes537).

Fig. 3 provides a typical set of current recordings under different conditions. The current expression of wild-type HCN4 is shown in Fig. 3A, as compared with the loss of current expression for D553N (Fig. 3B). Fig. 3 (C-E) shows the effects of individual Src, Fyn, and Yes on D553N current expression, respectively. Fig. $3(F-H)$ also shows the effects of Src+Fyn, Src+Yes, and Fyn+Yes on D553N current expression, respectively. Fig. $4 I$ shows the overall effects of combined Src+Fyn+Yes on D553N current expression. 
For effective comparison of the actions of Src kinases on D553N to HCN4, we calculated the current density and the activation kinetics at $-125 \mathrm{mV}$, which is near the fully activated voltage. Current density at the voltage in which all channels are open is directly related to our central interest of evaluating whether Src kinases can rescue the surface expression of D553N. The current densities under different conditions are shown in Fig. $4 A$.

Src and Yes, but not Fyn, can significantly rescue D553N current expression. Different combinations of three kinases all enhanced the current expression of D553N (Fig. 4A, asterisk). All three Src kinases expressed together (Fig. $3 I$ ) can restore $\sim 68 \%$ current expression of D553N as compared with the wild-type HCN4 expression (Fig. 4A, dark bars, HCN4: $37.15 \pm 3.21 \mathrm{pA} / \mathrm{pF}, n=7$; D553N+Src/Fyn/Yes: $25.25 \pm 2.17 \mathrm{pA} / \mathrm{pF}, n=$ 10) (Fig. 4A).

The effects of Src/Fyn/Yes kinases on the current activation kinetics are also different (Fig. $4 B$ ). The time constants for activation kinetics were obtained by fitting the onset current with one-exponential function at $-125 \mathrm{mV}$ under different conditions. Fyn accelerated but Yes slowed the activation kinetics of D553N, whereas Src had no effects ( $p^{*}$ values). The combinations of Src+Fyn and Fyn+Yes can accelerate D553N activation kinetics, whereas the combination of Src+Yes cannot. A combination of all three Src kinases can speed D553N activation kinetics at $-125 \mathrm{mV}$ (Fig. 4, $B$ and $D$ ); the time constants are $4.07 \pm 0.59 \mathrm{~s}(n=3)$ for $\mathrm{D} 553 \mathrm{~N}$ and $1.24 \pm 0.05 \mathrm{~s}(n=9)$ for $\mathrm{D} 553 \mathrm{~N} / \mathrm{Src} / \mathrm{Fyn} /$ Yes, respectively $(p=0.01)$. It is worth pointing out that in $16 \mathrm{D} 553 \mathrm{~N}$ transfected cells we studied only three cells that expressed time-dependent inward 
currents at $-125 \mathrm{mV}$ and that were used for calculating current density and activation kinetics shown in Fig. $4(A$ and $B)$. The other 13 cells expressed no currents at the test potentials ranging from $-75 \mathrm{mV}$ to $-135 \mathrm{mV}$. As a comparison, we also showed the statistical analysis of comparing Src kinases on D553N to HCN4 indicated by $p$ values in Fig. $4 B$.

To assess the overall functional rescuing effects of Src/Fyn/Yes on D553N gating, we examined the biophysical properties of D553N co-expressed with Src/Fyn/Yes (Fig. 3I) in comparison with the wild-type HCN4 (Fig. $3 A$ ). The Boltzmann equation best fit from the averaged activation curves obtained from tail currents showed a small depolarizing shift of D553N+Src/Fyn/Yes compared with $\mathrm{HCN} 4$ with no altered slope factors(s). However, statistical analysis from the means of two groups yielded no significant difference $\left(\mathrm{V}_{0.5}{ }_{-} \mathrm{HCN} 4:-86.1 \pm 1.5 \mathrm{mV}, n=7 ; \mathrm{V}_{0.5} \_\right.$D553N+Src/Fyn/Yes: $-81.6 \pm 1.4$ $\mathrm{mV}, n=9 ; p=0.0724$, s_HCN4: $8.2 \pm 0.2 \mathrm{mV}, n=7 ;$ s_D553N+Src/Fyn/Yes: $8.4 \pm 0.2$ $\mathrm{mV}, n=9, p=0.577$ ) (Fig. 4C). On the other hand, the effects of Src kinases on D553N activation kinetics are complex. Compared with the wild-type HCN4, the averaged activation kinetics for $\mathrm{D} 553 \mathrm{~N}+\mathrm{Src} / \mathrm{Fyn} / \mathrm{Yes}$ were slowed at the beginning of the activation curve, accelerated near the middle of the activation curve, but statistically indistinguishable at the potentials after the half-activation point (Fig. $4 D)(n=7$ for $\mathrm{HCN} 4, n=9$ for D553N+Src/Fyn/Yes). 
Enhanced Membrane Surface Expression of D553N by Src, Fyn, and Yes Tyrosine Kinases

We have previously shown that Src-mediated tyrosine phosphorylation increases the HCN2 and HCN4 channel activity via shifting the activation curve to depolarizing potentials (short term effect) and enhancing the cell surface expression (long term effect) (Arinsburg et al., 2006; Huang et al., 2008; Li et al., 2008; Yu et al., 2004). To seek further supporting evidence for rescuing surface expression of D553N by three Src kinases, we constructed the D553N-DsRed fusion protein and performed the confocal imaging experiments. Fig. 5 shows that most wild-type HCN4 is expressed on the cell surface (Fig. 5A). By contrast, most D553N cannot reach the cell surface (Fig. 5B). Coexpression with either Src (Fig. 5D) or Yes (Fig. 5F) can significantly enhance the surface expression of D553N. However, Fyn has been much less significant on D553N surface expression, as evidenced by a significant amount of D553N remaining in the cytosol (Fig. 5E). DsRed vector itself was uniformly expressed across the cell (Fig. 5C) and served as a negative control. These results, combined with the protein biochemistry analysis, collectively provide the cellular evidence for the functional rescue of D553N current expression at the plasma membrane by Src/Fyn/Yes kinases. 


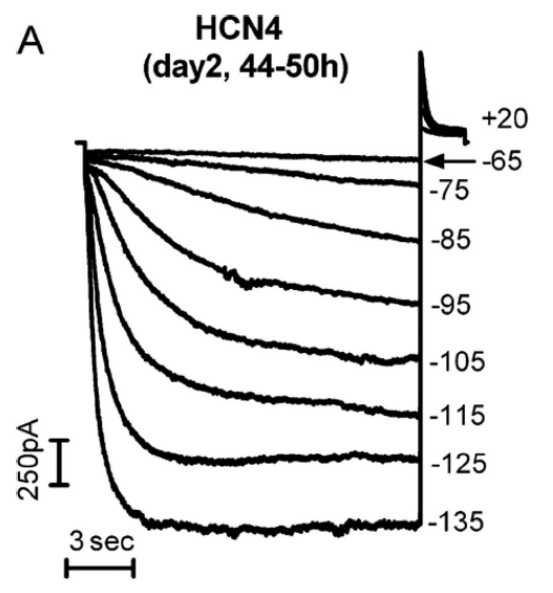

C

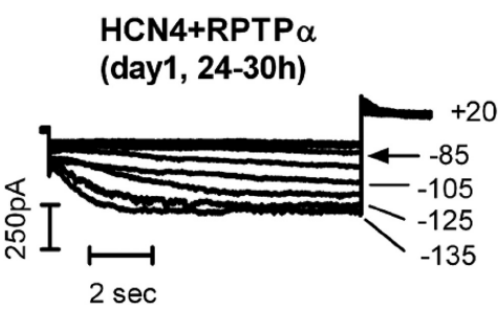

D HCN4+pRK5

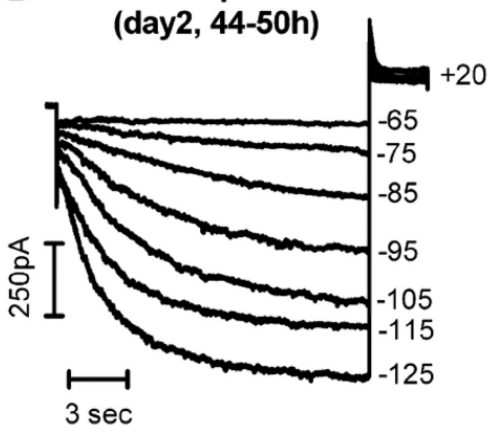

Fig 1. RPTPa on HCN4 current expression in HEK293 cells.

$A$, HCN4 current expression in response to 15 -s hyperpolarizing pulses from -65 to -135 $\mathrm{mV}$ in $10-\mathrm{mV}$ increments. Tail currents were recorded at $+20 \mathrm{mV}$. $B$, in a cell cotransfected with RPTP $\alpha$ after 1 day of incubation, HCN4 current expression in response to 10 -s hyperpolarizing pulses from -65 to $-135 \mathrm{mV}$ in $10-\mathrm{mV}$ increments. $C$, in a cell co-transfected with RPTP $\alpha$ after 2 days of incubation, HCN4 current expression in response to 10 -s hyperpolarizing pulses from -65 to $-135 \mathrm{mV}$ in $10-\mathrm{mV}$ increments. $D$, HCN4 current expression in HEK293 cells co-expressed with the empty vector, pRK5. 

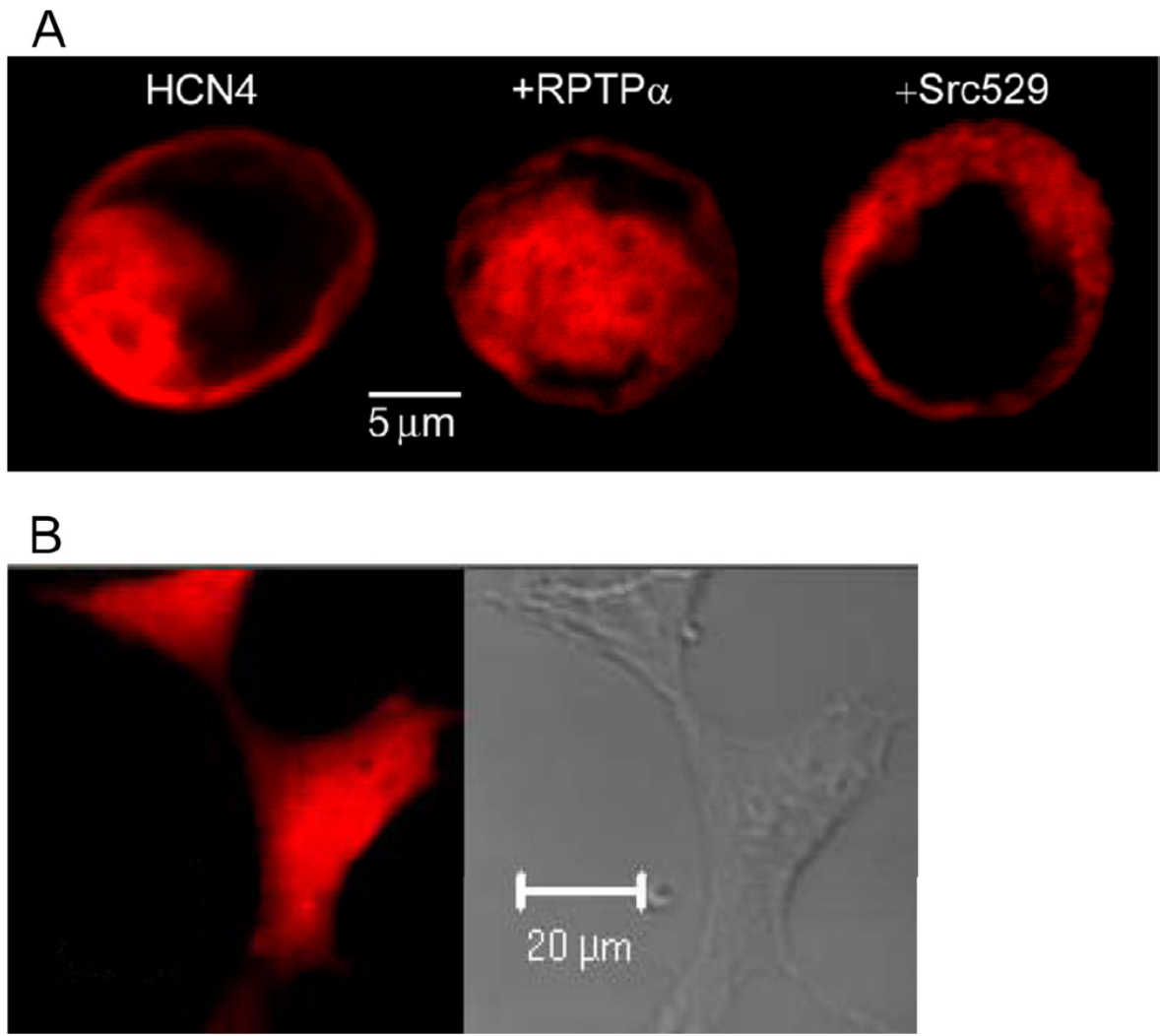

Fig 2. Src/RPTPa on HCN4 cell surface expression in HEK293 cells.

$A$, fluorescence imaging of HCN4-DsRed, HCN4-DsRed+RPTPa, and HCN4DsRed+Src529. B, fluorescence (left panel) and bright field (right panel) images of cells transfected with DsRed alone. 

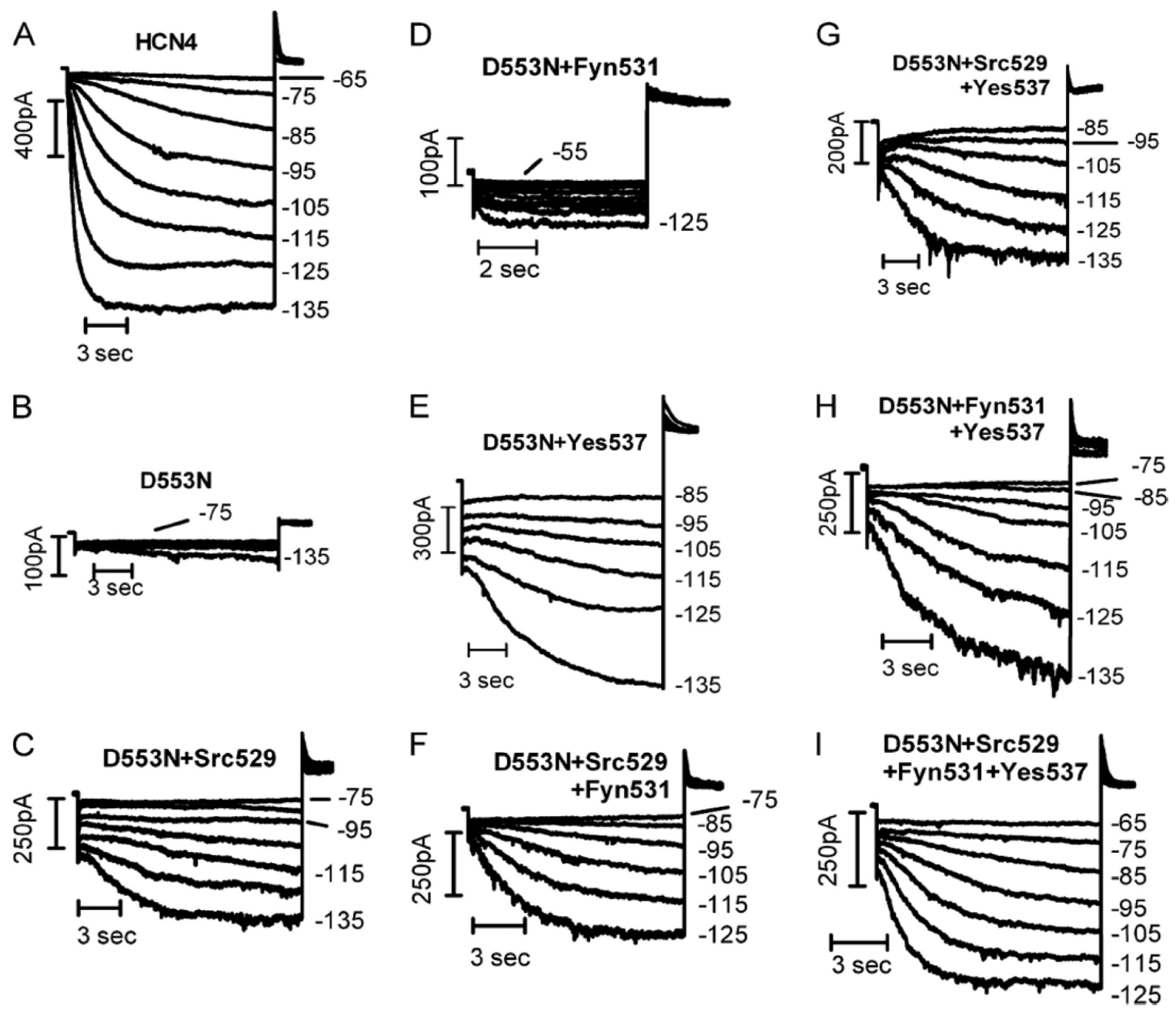

Fig 3. Src/Fyn/Yes kinases on current expression of D553N at $-125 \mathrm{mV}$.

Currents elicited by hyperpolarizing pulses in 10-mV increments are presented for HCN4
$(A), \quad \mathrm{D} 553 \mathrm{~N}(B), \quad \mathrm{D} 553 \mathrm{~N}+\mathrm{Src} 529$
(C), D553N+Fyn531
$(D), \quad \mathrm{D} 553 \mathrm{~N}+\mathrm{Yes} 537$
$(E)$
D553N+Src529+Fyn531 (F), D553N+Src529+Yes537 (G), D553N+Fyn531+Yes537 $(H)$, and D553N+Src529+Fyn531+Yes537 (I). The test potentials are labeled in the figures. The pulse durations were $15 \mathrm{~s}$ for $A-C, E$, and $G ; 12 \mathrm{~s}$ for $F, H$, and $I$; and $6 \mathrm{~s}$ for $D$. The holding potential was $-10 \mathrm{mV}$. 

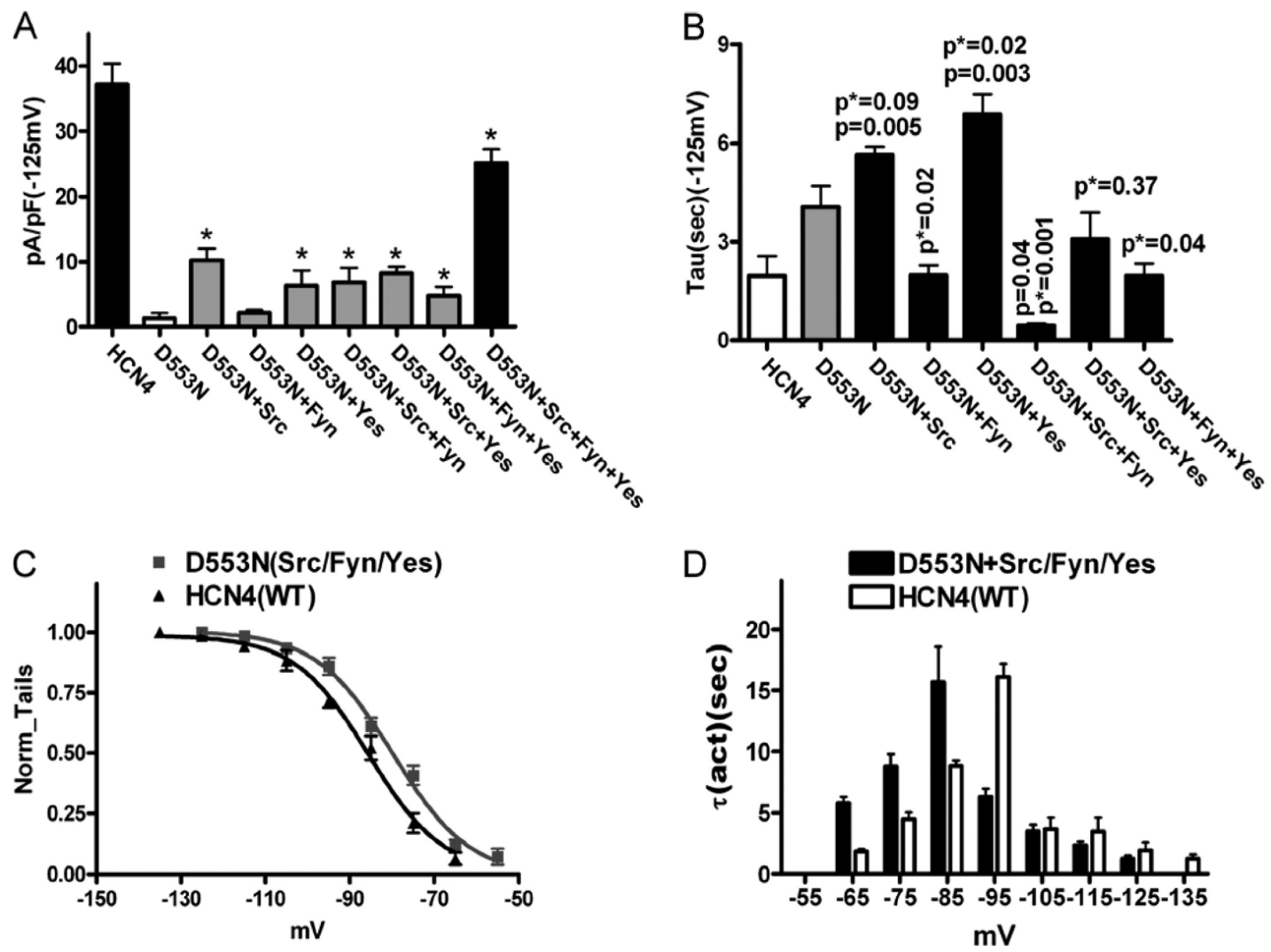

Fig 4. Src/Fyn/Yes kinases on current density and gating of D553N compared with HCN4. $A$, current density measured at $-125 \mathrm{mV}$ under different conditions. An asterisk indicates a statistically significant difference in comparison with D553N current density. $B$, time constants of activation kinetics at $-125 \mathrm{mV}$ under different conditions. $p$ values indicated statistical significance in comparing the effects of the kinases on D553N to HCN4 (white bar). $p^{*}$ values indicated statistical significance in comparing the effects of the kinases on D553N to D553N alone (gray bar). $C$, activation curves of HCN4 (WT) and D553N+Src/Fyn/Yes. D, activation kinetics of HCN4 (WT) and $\mathrm{D} 553 \mathrm{~N}+\mathrm{Src} / \mathrm{Fyn} /$ Yes. All of the statistical results were from seven to nine cells for each group except for D553N (only three cells expressed little currents of 16 cells tested). 


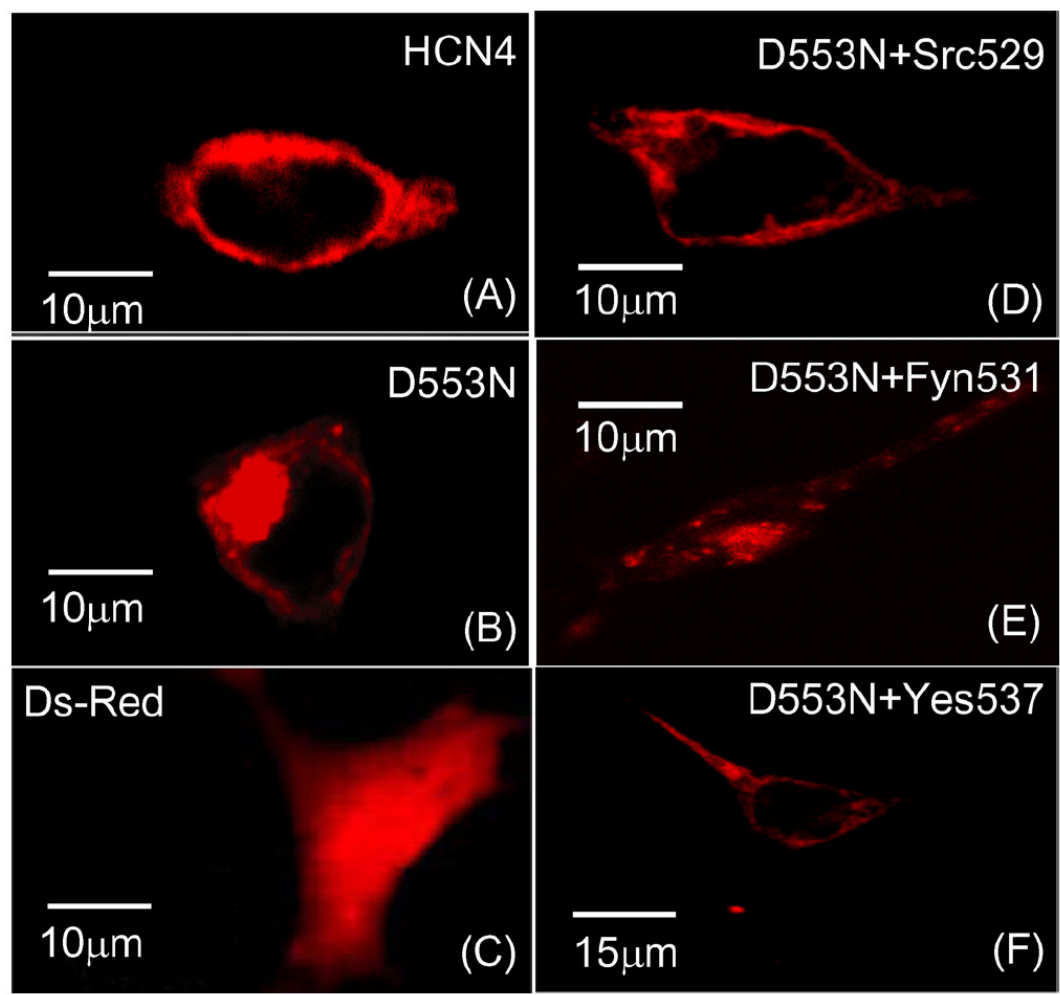

Fig 5. Src/Fyn/Yes kinases on D553N fluorescence imaging.

Fluorescence images of cells transfected with $\mathrm{HCN} 4(A), \mathrm{D} 553 \mathrm{~N}(B)$, DsRed vector $(C)$, D553N+Src529 (D), D553N+Fyn531 (E), and D553N+Yes537 $(F)$. All of the results were repeated in an additional eight to ten cells. 


\section{TABLE 1}

Relative roles of Src, Fyn, and Yes on D553N surface/current expression and activation kinetics

- , no or little expression or little effect or decrease; + , increase; ++ , significant increase or acceleration.

\begin{tabular}{lccc}
\hline & $\begin{array}{c}\text { Surface } \\
\text { expression }^{a}\end{array}$ & $\begin{array}{c}\text { Current } \\
\text { density }\end{array}$ & $\begin{array}{c}\text { Activation } \\
\text { kinetics }\end{array}$ \\
\hline D553N & - & - & \\
+ Src & ++ & ++ & - \\
+ Fyn & + & - & ++ \\
+ Yes & ++ & ++ & - \\
\hline
\end{tabular}

${ }^{a}$ Combined results of biotinylation and fluorescence imaging. 


\section{Discussion}

Defective trafficking of mutant channels represents an important mechanism for $\mathrm{Kv}$ channels causing long QT2 (Delisle et al., 2004). Studying long QT related Kv channel modulation has led to the findings that lower temperature and channel blockers can restore the surface and ionic current expression of the defective trafficking mutant channels (Delisle et al., 2004). In this work, we showed for the first time that by modulating the Src/Fyn/Yes kinase activity, a human HCN4 trafficking defective mutant D553N (also linked to long QT (Ueda et al., 2004)) can be rescued for normal surface and current expression. The corrected D553N exhibited the gating properties comparable with those of the wild-type HCN4 channels.

Enhanced tyrosine phosphorylation increased the activity of the cardiac pacemaker current, $I_{\mathrm{f}}$, in the sinoatrial node cells (Wu et al., 2000). Using genistein, a nonspecific tyrosine kinase inhibitor, we found a differential modulation of tyrosine phosphorylation

for HCN1, HCN2, and HCN4 expressed in Xenopus oocytes; genistein had no effects on HCN1 but reduced HCN2 or HCN4 current expression (Yu et al., 2004). In the case of HCN2, there was also a negative shift in the voltage dependence of activation that accompanied the current reduction. These studies represent the acute effects of altered tyrosine phosphorylation of $\mathrm{HCN}$ channel proteins on the gating properties of $I_{\mathrm{f}}$.

To investigate the long term effects of specific tyrosine kinases on HCN channels in a mammalian background, we studied the effects of Src kinase on HCN4 channel (the main isoform in the heart) expressed in HEK293 cells. We focused on the Src kinases for two reasons: it mediates epidermal growth factor receptor signaling (Thomas et al., 1997) and 
Src homology 3 domain was initially used to clone the first HCN channels (Santoro et al., 1997). We found that Src associated with and phosphorylated the HCN4 channel proteins, leading to the enhanced $\mathrm{HCN} 4$ current density near diastolic potentials (Arinsburg et al., 2006). This was the first direct evidence showing that 1) HCN4 channels can be phosphorylated by Src-mediated tyrosine kinases and 2) long term effects of tyrosine phosphorylation of HCN4 channels can induce the changes in the current density, which directly correlates with the number of functional channels expressed at the plasma membrane. Accompanying the increased current density were the accelerated activation kinetics and a positive shift in the voltage-dependent activation, which has been typically observed in the short term modulation of HCN4 channels. These conclusions were confirmed by the subsequent investigation on the major tyrosine residues that mediate Src actions (Li et al., 2008). Using PP2, a selective inhibitor of Src kinase family, we found that the reduced Src kinase activity can indeed shift the voltagedependent activation to hyperpolarizing potentials, an effect mediated by HCN4 Tyr ${ }^{531}$ (Li et al., 2008). Another tyrosine residue, $\mathrm{HCN} 4 \mathrm{Ty}^{554}$ previously reported by others (Zong et al., 2005), also contributed to the slowing of activation kinetics by PP2 (Li et al., 2008).

Work on the action of PP2 on HCN4 also resulted in two surprising observations. First, the PP2-induced negative shift of HCN4 voltage-dependent activation is not in agreement with our previous results with genistein. At least two factors can contribute to this discrepancy: mammalian cell (HEK293) versus amphibian (Xenopus oocytes) background and general (genistein) versus selective (PP2) inhibition of tyrosine kinases. Second, we found that PP2 also reduced the whole cell channel conductance (Li et al., 
2008). These results implied that even the short term effect of altered tyrosine phosphorylation may affect the number of functional channels at the plasma membrane.

More recently in the investigation of the potential role the tyrosine phosphatase might play in the modulation of $\mathrm{HCN}$ channel function, we found the dramatic inhibition of HCN2 current expression by RPTP $\alpha$ (Huang et al., 2008). The inhibited HCN2 current expression was due to the reduced surface expression of $\mathrm{HCN} 2$ channels via association between RPTP $\alpha$ and the HCN2 channel proteins, resulting in the channel dephosphorylation (Huang et al., 2008). The work demonstrated a previously unrecognized feature of $\mathrm{HCN}$ channel modulation by tyrosine phosphorylation: the tyrosine phosphorylation state of $\mathrm{HCN}$ channel proteins represents one important regulatory mechanism for the cell surface expression of the functional channels, which directly determines the current expression of functional HCN channels. This feature may be utilized to enhance the surface and ionic current expression of HCN mutant channel that cannot reach the plasma membrane for normal function.

Indeed, the evidence presented in this work showed that the enhanced tyrosine phosphorylation mediated by Src kinases can rescue the surface expression of D553N for normal channel function. What was unexpected, however, is the finding that three Src kinases that were ubiquitously expressed in the heart have different functional effects on D553N channel activity. In agreement with the previous studies, the Src kinase-mediated tyrosine phosphorylation is associated with the acceleration of channel activation kinetics (Arinsburg et al., 2006; Li et al., 2008; Zong et al., 2005). These differential effects by $\mathrm{Src} / \mathrm{Fyn} / \mathrm{Y}$ es on enhancing D553N expression and function are summarized in Table 1. 
The differential phosphorylation of both wild-type HCN4 and D553N channels by three Src kinases suggested that different tyrosine residues are involved in mediating each of the kinases. These results also suggested a possibility that D553N may undergo a protein misfolding that prevents the nearby tyrosine residues from being phosphorylated. Association of Src tyrosine kinases appears to partially correct the protein folding that leads to the exposure of key tyrosine residues for phosphorylation.

To understand the mechanism by which tyrosine phosphorylation used to restore the surface expression of D553N, we proposed a model utilizing the three-dimensional crystal structure of the C-linker region of $\mathrm{HCN} 2$ for the following three reasons. First, D553N mutation occurred in the C-linker. Second, there is a high homology (91.6\%) between $\mathrm{HCN} 2$ and $\mathrm{HCN} 4$ in the C-linker. Third, $\mathrm{HCN} 2$ is the only protein in the HCN family whose crystal structure of the C-linker has been solved (Zagotta et al., 2003). $\mathrm{HCN} 4 \mathrm{Asp}^{53}$ corresponds to $\mathrm{HCN} 2 \mathrm{Asp}^{475}$. Among many potential mechanisms responsible for the defective trafficking of HCN4-D553N, protein misfolding is an attractive one. We hypothesized that there may exist a potential electrostatic interaction between $\mathrm{Asp}^{475}$ and $\mathrm{Lys}^{472}$ of the $\mathrm{B}^{\prime}$ helix. The negatively charged $\mathrm{Asp}^{475}$ is spatially close to the positively charged $\mathrm{Lys}^{472}$, similar to the relative spatial locations of Lys ${ }^{472}$ and $\mathrm{Glu}^{502}$ of the $\mathrm{D}^{\prime}$ helix, which have been demonstrated to form a salt bridge critical in maintaining the local folding of C-linker (Zagotta et al., 2003). The putative D475N (equivalent to D553N in HCN4) mutation can cause the loss of a negative charge, which may change the intersubunit interaction between $\mathrm{Lys}^{472}$ and $\mathrm{Glu}^{502}$ to alter local folding of the C-linker structure. We noted that $\mathrm{Lys}^{472}$ is changed to $\mathrm{Arg}^{550}$ in HCN4. With a guanyl group, $\mathrm{Arg}^{550}$ is more capable than $\mathrm{Lys}^{472}$ in forming multiple electrostatic 
interactions with nearby residues having negative side chains. Therefore, in HCN4D553N mutant, the potential salt bridge of $\mathrm{Asp}^{553}$ with $\mathrm{Arg}^{550}$ could be disrupted, which affected the interaction between $\mathrm{Arg}^{550}$ and $\mathrm{Glu}^{580}\left(\mathrm{Glu}^{502}\right.$ in HCN2) that is critical in intersubunit contacts (Zagotta et al., 2003). Furthermore, the Src kinase-mediated tyrosine phosphorylation at $\mathrm{Tyr}^{554}$ residue near $\mathrm{Asp}^{553}$ could introduce a negatively charged phosphate group, which could mimic the effect of $\mathrm{Asp}^{553}$ to rebuild the salt bridge interaction between $\mathrm{Arg}^{550}$ and $\mathrm{Glu}^{580}$, consistent with the previous studies demonstrating the importance of $\mathrm{Tyr}^{554}$ (Li et al., 2008; Zong et al., 2005). For wild-type $\mathrm{HCN} 4$, the existing negative charge on $\mathrm{Asp}^{553}$ might repel the entry of a phosphate group and limit the phosphorylation on certain nearby tyrosine residues.

While presenting a novel mechanism to correct the surface expression of a trafficking defective HCN4 mutant channel, we left at least three questions unanswered. First, what are the tyrosine residue(s) in HCN4 channel proteins that mediate the actions of Fyn and Yes? The same tyrosine residues (such as $\mathrm{Tyr}^{531}$ and $\mathrm{Tyr}^{554}$ ) are unlikely to be used by all three kinases. Fyn, not Src, accelerated D553N activation kinetics (Fig. 5B). Fyn may target the tyrosine residues in or near $\mathrm{A}^{\prime}$ and $\mathrm{B}^{\prime}$ helices of the $\mathrm{C}$-linker. On the other hand, Yes may phosphorylate different tyrosine residues that are located outside of the Clinker, which can explain its lack of acceleration in the activation kinetics. Second, what is the correlation, if any, among the surface expression and activation kinetics and the tyrosine phosphorylation state of HCN4 channels? Fyn showed acceleration of the channel activation kinetics but little effect on promoting the channel surface expression. Yes slowed the channel activation kinetics, but exhibited the most potency of promoting the cell surface expression of D553N (Table 1). It might involve other unknown proteins 
yet to be identified. Third, will an increase in the endogenous Src/Fyn/Yes kinase activity in myocytes help promote the surface expression of D553N in vivo? Addressing these questions represents our future research endeavors, leading to the discovery of an effective endogenous regulatory mechanism to correct cardiac arrhythmias caused by HCN channel mutants. 


\title{
Part II: Novel mechanism for suppression of hyperpolarization-activated cyclic nucleotide-gated pacemaker channels by receptor-like tyrosine phosphatase-alpha
}

\begin{abstract}
Tyrosine phosphorylation plays a pivotal role in the regulation of membrane trafficking of HCN channels. Previous reports have demonstrated that both of the $I_{\mathrm{f}}$ densities and gating properties were significantly enhanced by increase of tyrosine phosphorylation level in rat myocytes (Wu et al., 2000). Here, we report that a member of receptor-type tyrosine phosphatase, RPTP $\alpha$, can abolish the current expression of HCN2. Our results indicated that RPTP $\alpha$ plays the novel role in the regulation of $\mathrm{HCN}$ channel function via tyrosine dephosphorylation of HCN channel protein.
\end{abstract}




\section{Introduction}

Activated by membrane hyperpolarization, the $\mathrm{HCN} 2$ channels are important to rhythmic activity in neurons and myocytes (Robinson et al., 2003; Santoro et al., 2003). Accumulating evidence has also suggested an important role of tyrosine phosphorylation in modulating HCN channels (Wu et al., 2000; Yu et al., 2004). We have recently shown that increased tyrosine phosphorylation state of HCN4 by activated Src tyrosine kinase can enhance the gating of HCN4 channels (Arinsburg et al., 2006). Given that the efficacy of tyrosine phosphorylation is determined by the dynamic balance of tyrosine kinases and tyrosine phosphatases, reduced tyrosine phosphatase activity is speculated to increase HCN channel activity. Receptor-like protein-tyrosine phosphatases (RPTPs) are transmembrane phosphatases critical in cell growth and cell adhesion (Stoker, 2005). One member of RPTPs, RPTP $\alpha$, has been proposed to be a positive regulator of Src tyrosine kinases (Ponniah et al., 1999; Su et al., 1999). As a protein-tyrosine phosphatase, RPTP $\alpha$ should also be able to dephosphorylate phosphotyrosines of channel proteins. This work was designed to investigate whether RPTP $\alpha$ can inhibit the HCN channel activity by decreasing the tyrosine phosphorylation state of $\mathrm{HCN}$ channel proteins. 


\section{Results}

\section{RPTP $\alpha$ Inhibition of HCN2 Currents in HEK293 Cells}

Our recent discovery showed that increased tyrosine phosphorylation of HCN4 channel by activated Src tyrosine kinase can enhance the channel gating properties in HEK293 cells (Arinsburg et al., 2006; Li et al., 2008). We wondered whether increased tyrosine dephosphorylation by RPTP $\alpha$ may inhibit the gating properties of HCN channels expressed in HEK293 cells.

Functional expressions of HCN2 after 2 days (44-50 h) of transfection are shown in Fig 1. HCN2 currents were elicited by hyperpolarizing pulses detailed in the figure legends. Typical biophysical properties of the expressed channels such as the threshold activation, activation kinetics, and current densities are comparable with those reported previously (Arinsburg et al., 2006; Li et al., 2008; Zong et al., 2005). Co-transfection with RPTP $\alpha$, however, resulted in a dramatic inhibition (Fig. 1B) or a surprising loss of HCN2 currents (Fig. 1C). Similar results were reproduced in 10 additional cells for each HCN2 channel co-expressed with RPTP $\alpha$. As part of control experiments, the empty pRK5 vector did not affect $\mathrm{HCN} 2$ expression from 1 to 4 days post-transfection. Fig. $1 D$ shows the HCN2 recordings in a HEK293 cell co-transfected with HCN2 and pRK5 vector after 2 days. Similar results were obtained in an additional five cells.

Interestingly, we found the degree by which RPTP $\alpha$ inhibited HCN2 current expression changed with time. Fig. 2 shows HCN2 current expression recorded in HEK293 cells cotransfected with RPTP $\alpha$ after 1 day (24-30 h) (A), 2 days (44-50 h) (B), and 4 days $(C)$ post-transfection. Compared with HCN2 alone (Fig. 1A), co-transfection with RPTP $\alpha$ 
reduced $\mathrm{HCN} 2$ current density $(\mathrm{pA} / \mathrm{pF})$ measured at $-125 \mathrm{mV}$ by $64 \%$ after day 1 (HCN2 $=38.9 \pm 1.7, n=11 ; \mathrm{RPTP} \alpha=1.37 \pm 0.5, n=10)$, by $96 \%$ after day $2(\mathrm{RPTP} \alpha=1.5 \pm$ $0.5, n=10)$, and by $80 \%$ after day $4(\operatorname{RPTP} \alpha=7.7 \pm 0.7, n=10)$ (Fig. $2 D)$. The timedependent inhibition is not only significant as compared with the control, but also significant among groups (e.g. day 1 and day 2; day 2 and day 4 ). 

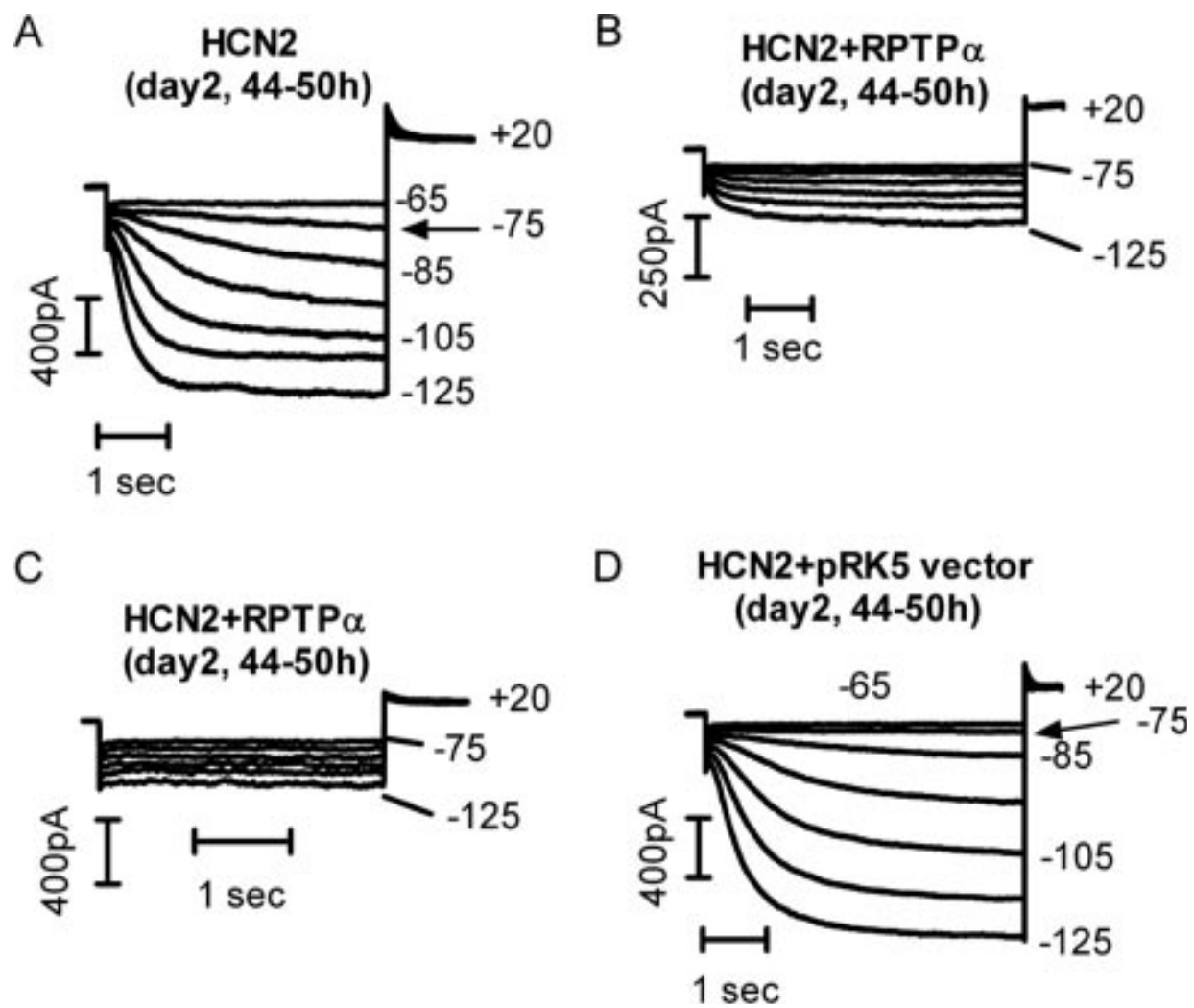

Fig 1. RPTPa inhibition of HCN2 current expression.

HCN2 currents were recorded 2 days after cell transfection. $A, \mathrm{HCN} 2$ currents in a HEK293 cell expressing HCN2 alone. Hyperpolarizing pulses (4 s) from -65 to $-125 \mathrm{mV}$ were applied. $B$, hyperpolarization-activated currents in a HEK293 cell co-transfected with HCN2 and RPTP $\alpha$. Hyperpolarizing pulses $(5 \mathrm{~s})$ from -75 to $-125 \mathrm{mV}$ were applied. $C$, hyperpolarization-activated currents in a HEK293 cell co-transfected with HCN2 and RPTP $\alpha$. Hyperpolarizing pulses (3 s) from -75 to $-125 \mathrm{mV}$ were applied. $D$, HCN2 currents in a HEK293 cell co-transfected with HCN2 and the empty pRK5 vector. Hyperpolarizing pulses (5 s) from -65 to $-125 \mathrm{mV}$ were applied. The tail currents were recorded at $+20 \mathrm{mV}$. The holding potential was $-10 \mathrm{mV}$. Arrows in $A$ and $D$ indicate the threshold activation of $\mathrm{HCN} 2$ currents. 

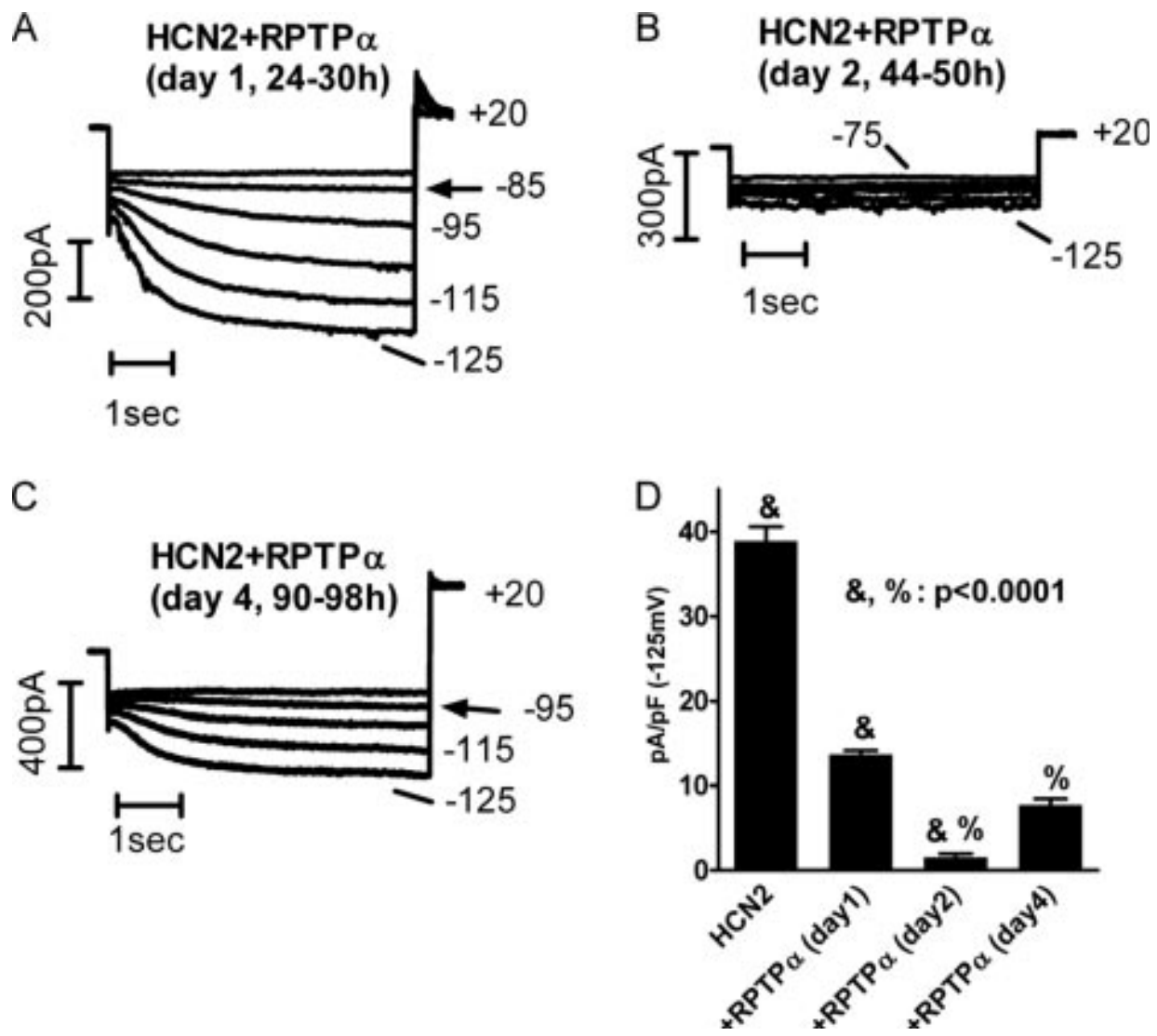

Fig 2. RPTPa induced time-dependent inhibition of $\mathrm{HCN} 2$ current expression. Hyperpolarizing pulses of $5 \mathrm{~s}$ ranging from -75 to $-125 \mathrm{mV}(A$ and $B$ ) or from 85 to -125 $\mathrm{mV}(C)$ in $10-\mathrm{mV}$ increments were applied to elicit HCN2 currents in HEK293 cells cotransfected HCN2 with RPTP $\alpha$ after day $1(A)$, day $2(B)$, and day $4(C)$. D, averaged $\mathrm{HCN} 2$ current density at $-125 \mathrm{mV}$ for the corresponding time periods. 


\section{Discussion}

In this study, we provided evidence showing dramatic inhibitory effects of tyrosine dephosphorylation by RPTP $\alpha$ on HCN2 channels. Two mechanisms are likely involved as follows: tyrosine dephosphorylation and membrane trafficking of $\mathrm{HCN}$ channels. Both are mediated by RPTP $\alpha$.

In HEK293 cells co-expressing HCN2 channels with RPTP $\alpha$ for 2 days yielded surprising inhibition or even elimination of the current expression. There are two plausible explanations as follows: the channels were retained in the cytoplasm leading to little or no expression of functional channels on plasma membrane, or gating properties of the channels on the plasma membrane were inhibited. In this study, we also performed western blot analysis on the membrane fraction of cells and revealed that the constitutively active Src, which increases the tyrosine phosphorylation level of the channels, increased the surface expression of the channels. On the other hand, RPTP $\alpha$, which decreases the tyrosine phosphorylation level of the channels, retained most channels in the cytoplasm.

It is surprising that the HCN2 channel expression was largely blocked by RPTP $\alpha$ after 2 days of transfection and reappeared after 4 days of transfection (Fig. $2 B$ ). It offers a likely explanation to no measurable or much smaller time-dependent inward currents in HEK293 cells co-transfected by HCN2 with RPTP $\alpha$ for the same time periods (Fig. 1, $B$ and $C$ ). It is worth noting that whole-cell patch clamp technique applied to individual cells is more sensitive than Western blotting, which obtains the average result from batch 
of cells. Therefore, after transfection for 2 days we were able to detect small current expression in some cells, but not in protein expression.

Protein-tyrosine phosphatases, like protein-tyrosine kinases, play a critical role in the regulation of physiological events (Ponniah et al., 1999; Su et al., 1999). RPTP $\alpha$ has a short extracellular domain (about 123-150 amino acids long) that contains eight potential $N$-glycosylation sites (Daum et al., 1994; Kaplan et al., 1990). Following a transmembrane region, there are two tandem domains having phosphatase catalytic activity. RPTP $\alpha$ is expressed in two isoforms differing by nine residues (Kaplan et al., 1990) that are highly glycosylated, p100 and p130 on SDS-PAGE (Daum et al., 1994). The p100 form contains only $N$-linked glycosylation, whereas p130 contains both $N$ linked and $O$-linked.

Our data have shown a critical role that RPTP $\alpha$ plays in the tyrosine dephosphorylation of HCN2 channels. The short-term likely involves the tyrosine dephosphorylation, is the reduced $\mathrm{I}_{f}$ activity in cardiac myocytes. The long-term effects (days) include the inhibition of HCN channel surface expression and possibly channel biosynthesis. Recently, HCN4 mutants have been linked to the bradycardia and long-QT arrhythmias (Milanesi et al., 2006; Nof et al., 2007; Schulze et al., 2003; Ueda et al., 2004). The common cellular mechanism was retaining membrane trafficking caused by the truncated HCN4 protein lacking cyclic nucleotide binding domain (Schulze et al., 2003), D553N in the C-linker between S6 and cyclic nucleotide binding domain (Ueda et al., 2004), and G480R in the channel pore region (Nof et al., 2007). The evidence we presented in this work provided a novel mechanism that may be used to enhance the surface expression of mutant HCN channels for effecting normal cardiac pacemaker activity. 


\section{Chapter 3}

\section{Inactivation of L-type calcium channel modulated by HCN2 channel}

The goal of this chapter is to explore a new role of $\mathrm{HCN}$ serving as a regulatory protein to regulate the $\mathrm{Ca}^{2+}$ influx via the control of L-type calcium channel (LTCC) inactivation. The results in this chapter have been published (Am J Physiol Cell Physiol 298(5): C1029-37, 2010). 


\begin{abstract}
$\mathrm{Ca}^{2+}$ entry is delicately controlled by inactivation of L-type calcium channel (LTCC) composed of the pore-forming subunit $\alpha 1 \mathrm{C}$ and the auxiliary subunits $\beta 1$ and $\alpha 2 \delta$. Calmodulin is the key protein that interacts with the $\mathrm{COOH}$-terminal motifs of $\alpha 1 \mathrm{C}$, leading to the fine control of LTCC inactivation. In this study we show evidence that a hyperpolarization-activated cyclic nucleotide-gated channel, HCN2, can act as a nonchannel regulatory protein to narrow the L-type $\mathrm{Ca}^{2+}$ channel current-voltage curve. In the absence of LTCC auxiliary subunits, HCN2 can induce $\alpha 1 \mathrm{C}$ inactivation. Without $\alpha 2 \delta$, HCN2-induced fast inactivation of $\alpha 1 \mathrm{C}$ requires calmodulin. With $\alpha 2 \delta$, the $\alpha 1 \mathrm{C} / \mathrm{HCN} 2 / \alpha 2 \delta$ channel inactivation does not require calmodulin. In contrast, $\beta 1$-subunit plays a relatively minor role in the interaction of $\alpha 1 \mathrm{C}$ with $\mathrm{HCN} 2$. $\mathrm{The}^{\mathrm{N}} \mathrm{NH}_{2}$ terminus of $\mathrm{HCN} 2$ and the IQ motif of $\alpha 1 \mathrm{C}$ subunit are required for $\alpha 1 \mathrm{C} / \mathrm{HCN} 2$ channel interaction. $\mathrm{Ca}^{2+}$ channel inactivation is significantly slowed in hippocampus neurons (HNs) overexpressing $\mathrm{HCN} 2$ mutant lacking $\mathrm{NH}_{2}$ terminus and accelerated in $\mathrm{HNs}$ overexpressing the wild-type HCN2 compared with HN controls. Collectively, these results revealed a potentially novel protection mechanism for achieving the LTCC inactivation via interaction with $\mathrm{HCN} 2$.
\end{abstract}




\section{Introduction}

L-TYPE CALCIUM CHANNEL (LTCC) current inactivation is dependent on both $\mathrm{Ca}^{2+}$ ions and voltage (Cens et al., 2006; Liang et al., 2003). The $\beta$ - and $\alpha 2 \delta$-subunits regulate the membrane expression and the voltage-dependent inactivation of the $\alpha 1 \mathrm{C}$-subunit (Arikkath et al., 2003). Calmodulin (CaM) plays a central role in both $\mathrm{Ca}^{2+}$-dependent inactivation (CDI) and voltage-dependent inactivation (VDI) using $\mathrm{Ba}^{2+}$ as the charge carrier (Kim et al., 2004; Liang et al., 2003). CaM is proposed to tether with the IQ motif in the $\mathrm{COOH}$ terminus of $\alpha 1 \mathrm{C}$ (Peterson et al., 1999; Pitt et al., 2001). $\mathrm{Ca}^{2+}$ influx through LTCC activates apoCaM $\left(\mathrm{Ca}^{2+}\right.$-free $\left.\mathrm{CaM}\right) / \mathrm{IQ}$ complex. The activated CaM/IQ triggers a conformational change of the inactivation machinery consisting of $\alpha 1 \mathrm{C} \mathrm{COOH}-$ terminal domains such as peptide A, peptide C (or CB), IQ motif, and CaM. As a result, the inactivation machinery rapidly blocks the inner surface of the pore, leading to fast CDI (Erickson et al., 2003; Pitt et al., 2001). CaM also has been proposed to interact with the linker of the transmembrane I and II segments associated with the $\beta 1$-subunit to modulate VDI (Cens et al., 2006; Kim et al., 2004).

Hyperpolarization-activated, cyclic nucleotide-gated $(\mathrm{HCN})$ channels are activated by membrane hyperpolarization, generating a voltage- and time-dependent inward current called $I_{\mathrm{h}}$ or $I_{\mathrm{f}}$, which has been traditionally thought to permeate both $\mathrm{Na}^{+}$and $\mathrm{K}^{+}$ions (DiFrancesco et al., 1993). Recently, we discovered that $\mathrm{Ca}^{2+}$ ions also can permeate HCN channels, even in a small percentage of the total current (Yu et al., 2007). Furthermore, activation of $I_{\mathrm{h}}$ and $I_{\mathrm{f}}$ can increase the synapse transmission in dorsal root ganglion neurons (Yu et al., 2004) and shorten the action potential duration in ventricular 
myocytes (Yu et al., 2007), respectively. However, the underlying mechanisms are not well understood. In the present work, we focus on a hypothesis that a closed HCN channel can interact with LTCC at the plasma membrane, leading to the fast inactivation of L-type $\mathrm{Ca}^{2+}$ current $\left(I_{\mathrm{CaL}}\right)$. 


\section{Results}

\section{CDI and VDI of $\alpha 1 C / \alpha 2 \delta / \beta 1$ and $\alpha 1 C / \alpha 2 \delta / \beta 1 / H C N 2$ channels in HEK-293 cells}

Figure $1 A$ exemplified a well-established difference between CDI and VDI: faster inactivation for $I_{\mathrm{Ca}}$ (solid line) than for $I_{\mathrm{Ba}}$ (shaded line), recorded at $+10 \mathrm{mV}$. This difference, however, is largely diminished in the presence of HCN2 (Fig. 1B): like $I_{\mathrm{Ca}}$, the $I_{\mathrm{Ba}}$ of $\alpha 1 \mathrm{C} / \alpha 2 \delta / \beta 1 / \mathrm{HCN} 2$ can inactivate almost completely to the closed state. Figure $1 C$ summarizes the time constants of inactivation of $I_{\mathrm{Ca}}$ and $I_{\mathrm{Ba}}$ for $\alpha 1 \mathrm{C} / \alpha 2 \delta / \beta 1$ and $\alpha 1 \mathrm{C} / \alpha 2 \delta / \beta 1 / \mathrm{HCN} 2$. They are $25.0 \pm 3.2 \mathrm{~ms}$ for $I_{\mathrm{Ca}}$ and $228.5 \pm 24.2 \mathrm{~ms}$ for $I_{\mathrm{Ba}}$ of

$\alpha 1 \mathrm{C} / \alpha 2 \delta / \beta 1$, and $58.3 \pm 3.3 \mathrm{~ms}$ for $I_{\mathrm{Ca}}$ and $72.5 \pm 5.6 \mathrm{~ms}$ for $I_{\mathrm{Ba}}$ of $\alpha 1 \mathrm{C} / \alpha 2 \delta / \beta 1 / \mathrm{HCN} 2$. The difference between $I_{\mathrm{Ca}}$ and $I_{\mathrm{Ba}}$ of $\alpha 1 \mathrm{C} / \alpha 2 \delta / \beta 1 / \mathrm{HCN} 2$ inactivation kinetics was diminished compared with that between $I_{\mathrm{Ca}}$ and $I_{\mathrm{Ba}}$ of $\alpha 1 \mathrm{C} / \alpha 2 \delta / \beta 1$ inactivation kinetics, indicating that $\mathrm{HCN} 2$ can help complete the $I_{\mathrm{Ba}}$ inactivation.

We also examined whether the current-voltage relationship curves of $\alpha 1 \mathrm{C} / \alpha 2 \delta / \beta 1$ are altered by $\mathrm{HCN} 2$. Representative $I_{\mathrm{Ca}}$ are shown for $\alpha 1 \mathrm{C} / \alpha 2 \delta / \beta 1$ (Fig. $1 D$ ) and $\alpha 1 \mathrm{C} / \alpha 2 \delta / \beta 1 / \mathrm{HCN} 2$ (Fig. $1 E$ ). In the presence of $\mathrm{HCN} 2$, there was a positive shift in the threshold activation of $I_{\mathrm{CaL}}$ and more reduced current amplitude at the depolarized end of the bell shape near $+50 \mathrm{mV}$ (Fig. $1 F$, shaded line). All results were repeated in an additional five to eight cells. 


\section{Fast inactivation of $\alpha 1 \mathrm{C} / \mathrm{HCN} 2$ channels in HEK-293}

To examine a possible hidden interaction between $\alpha 1 \mathrm{C}$ and $\mathrm{HCN} 2$, we studied $\alpha 1 \mathrm{C}$ inactivation by coexpressing $\alpha 1 \mathrm{C}$ and $\mathrm{HCN} 2$ in the absence of auxiliary subunits. With the use of $\mathrm{Ba}^{2+}$ as the charge carrier, $\alpha 1 \mathrm{C}$ exhibited little current inactivation when expressed alone (Fig. $2 A$, top). The auxiliary subunit $\alpha 2 \delta$ could limit the $\mathrm{Ca}^{2+}$ influx by inducing a slow inactivation of $\alpha 1 \mathrm{C}$ (Fig. $2 A$, top). Figure $2 B$, bottom, shows the absence of endogenous time-dependent inward currents in HEK-293 cells without cDNA transfection under our experimental conditions. These results are in agreement with the previous reports (Felix et al., 1997; Yasuda et al., 2004). However, when HCN2 was coexpressed with $\alpha 1 \mathrm{C}$, a fast inactivation of $\alpha 1 \mathrm{C} / \mathrm{HCN} 2$ was observed (Fig. $2 B$, top). This new inactivation has a differential sensitivity for $\mathrm{Ba}^{2+}$ and $\mathrm{Ca}^{2+}$ ions, capable of coming back to the closed state in the presence of $\mathrm{Ca}^{2+}$ (shaded line) but not $\mathrm{Ba}^{2+}$ (solid line), the typical characteristics of the conventional LTCC inactivation (Kim et al., 2004; Zuhlke et al., 1998). Adding $\alpha 2 \delta$ did not significantly affect the $\alpha 1 \mathrm{C} / \mathrm{HCN} 2$ inactivation (Fig. $2 A$, bottom). With $\mathrm{Ba}^{2+}$ as the charge carrier, time constants for inactivation of $\alpha 1 \mathrm{C} / \alpha 2 \delta$, $\alpha 1 \mathrm{C} / \mathrm{HCN} 2$, and $\alpha 1 \mathrm{C} / \mathrm{HCN} 2 / \alpha 2 \delta$ were $156 \pm 19,26 \pm 4$, and $22 \pm 2 \mathrm{~ms}$, respectively $(n=$ 8). The inactivation for $\alpha 1 \mathrm{C} / \alpha 2 \delta$ channels was significantly slower than that for $\alpha 1 \mathrm{C} / \mathrm{HCN} 2$ or $\alpha 1 \mathrm{C} / \mathrm{HCN} 2 / \alpha 2 \delta(P=0.002, n=8)$. There was no statistical difference between $\alpha 1 \mathrm{C} / \mathrm{HCN} 2$ and $\alpha 1 \mathrm{C} / \mathrm{HCN} 2 / \alpha 2 \delta$ channel inactivation $(P=0.5, n=8)$. To verify that the current is via the conventional $\alpha 1 \mathrm{C}$ channel, verapamil $(10 \mu \mathrm{M})$ was applied to block the current activation (Fig. 2, $A$, bottom, and $B$, top). 
To address the concern that the fast inactivation of $\alpha 1 \mathrm{C} / \mathrm{HCN} 2$ or $\alpha 1 \mathrm{C} / \mathrm{HCN} 2 / \alpha 2 \delta$ channel was probably due to the contamination from the deactivation of HCN2 channels, we studied current expression of HCN2 in the external solution normally used for characterization of $\mathrm{HCN} 2$ and in the external solution used to record $I_{\mathrm{Ba}}$ inactivation (e.g., Fig. 2, $A$ and $B$ ). The hyperpolarization-activated $\mathrm{HCN} 2$ channels permeate both $\mathrm{Na}^{+}$and $\mathrm{K}^{+}$ions (DiFrancesco et al., 1993). The channel cannot be activated in the absence of external $\mathrm{Na}^{+}$and $\mathrm{K}^{+}$ions (DiFrancesco et al., 1993). In the external solution containing $140 \mathrm{mM} \mathrm{Na}^{+}$and $30 \mathrm{mM} \mathrm{K}^{+}$, a typical HCN2 current (the time-dependent inward current) was recorded in response to a 3 -s hyperpolarizing pulse to $-125 \mathrm{mV}$ (Fig. $2 C$ ), consistent with the previous report (Yu et al., 2007). When the $\mathrm{Na}^{+}$and $\mathrm{K}^{+}$ions were removed from the external solution, no time-dependent inward current was recorded (Fig. 2C).

HCN2 channels are closed at membrane depolarization that is used to activate LTCC (DiFrancesco et al., 1993). In response to a depolarization to $+10 \mathrm{mV}$ from the holding potential of $-90 \mathrm{mV}$, there was no $\mathrm{HCN} 2$ activation current in the solution containing 140 $\mathrm{mM} \mathrm{Na}^{+}$and $30 \mathrm{mM} \mathrm{K}$ (Fig. $2 D$ ). The inactivation of $\alpha 1 \mathrm{C} / \mathrm{HCN} 2 / \alpha 2 \delta$ channel in the $\mathrm{Na}^{+}$- and $\mathrm{K}^{+}$-free solution was used as a comparison (Fig. 2D). These data strongly indicate that the closed HCN2 channels can act as the nonfunction channel to induce a fast inactivation of $\alpha 1 \mathrm{C}$.

\section{Role of $\mathrm{Ca}^{2+}$-free $\mathrm{CaM}$ in $\alpha 1 \mathrm{C} / \mathrm{HCN} 2$ channel inactivation}

Given the pivotal role CaM plays in the $\mathrm{Ca}^{2+}$ channel inactivation, we tested the idea that $\mathrm{CaM}$ may be involved in the fast inactivation of $\alpha 1 \mathrm{C} / \mathrm{HCN} 2$ channel for two reasons:

$\mathrm{CaM}$ is endogenously present in HEK-293 cells, and apoCaM (no $\mathrm{Ca}^{2+}$-bound $\mathrm{CaM}$ ) is 
tethered to structural domains in the $\mathrm{COOH}$ terminus of $\alpha 1 \mathrm{C}$ subunit (Erickson et al., 2003; Pitt et al., 2001).

We first used two structurally different calmodulin inhibitors, W-7 [N-(6-aminohexyl)-5chloro-1-naphthalenesulfonamide] (Hidaka et al., 1981) and trifluoperazine (TFP) (Reinhart et al., 1980), to study their potential effects on $\alpha 1 \mathrm{C} / \mathrm{HCN} 2$ channel inactivation. After binding $\mathrm{Ca}^{2+}$ ions, $\mathrm{CaM}$ undergoes a conformational change leading to the exposure of its hydrophobic activation site for binding to its substrate, CaM-dependent kinase (CaMK). Both W-7 and TFP block CaM actions by occupying the hydrophobic site to prevent binding of CaMK (Hidaka et al., 1981; Osawa et al., 1998). Figure $3 A$ shows that both drugs at $15 \mu \mathrm{M}$ eliminated the fast inactivation of $\alpha 1 \mathrm{C} / \mathrm{HCN} 2$ channels within $15-20$ min, consistent with the time course of inhibiting CaM activity by both drugs. The effect of the drug was observed in the same cell. The similar results were obtained in an additional five to seven cells.

We then employed a dominant negative calmodulin mutant, $\mathrm{CaM}_{(1,2,3,4)}$ (Xia et al., 1998). Compared with the current expression of $\alpha 1 \mathrm{C} / \mathrm{HCN} 2$ coexpressed with a wild-type calmodulin, $\mathrm{CaM}_{(\mathrm{WT})}$, overexpression of $\mathrm{CaM}_{(1,2,3,4)}$ eliminated the fast inactivation of a1C/HCN2 (Fig. 3B, shaded line). These pharmacological and mutant CaM data strongly suggested that the interaction of $\alpha 1 \mathrm{C}$ and $\mathrm{HCN} 2$ is partially mediated by CaM. 


\section{Role of $\alpha 2 \delta$ in $\alpha 1 \mathrm{C} / \mathrm{HCN} 2$ channel inactivation}

Nearly all studies of CaM-mediated $\alpha 1 \mathrm{C}$ inactivation, carried out in either Xenopus oocytes or mammalian cells (HEK-293, Chinese hamster ovary, COS), have been performed with coexpression of $\beta 1$ - and $\alpha 2 \delta$-subunits. This is due to the important roles of $\beta 1$ - and $\alpha 2 \delta$-subunits in expression of $\alpha 1 \mathrm{C}$ on plasma membrane and $\mathrm{Ca}^{2+}$ channel function (Arikkath et al., 2003). Previous studies also have demonstrated a significant role of $\alpha 2 \delta$ in initiating the inactivation of $\alpha 1 \mathrm{C}$ expressed in HEK-293 cells (Felix et al., 1997; Yasuda et al., 2004). Wondering whether CaM would have the similar impact on the $\alpha 1 \mathrm{C} / \mathrm{HCN} 2$ channel inactivation in the presence of the $\alpha 2 \delta$ subunit, we tested the effects of $\mathrm{W}-7$ and TFP on the current expression of $\alpha 1 \mathrm{C} / \mathrm{HCN} 2 / \alpha 2 \delta$ channels. Surprisingly, neither W-7 (left) nor TFP (right) affected the inactivation of $\alpha 1 \mathrm{C} / \mathrm{HCN} 2 / \alpha 2 \delta$ channels (Fig. $3 C$, shaded lines). To further confirm this observation, we overexpressed either $\mathrm{CaM}_{(\mathrm{WT})}$ or $\mathrm{CaM}_{(1,2,3,4)}$ with $\alpha 1 \mathrm{C} / \mathrm{HCN} 2 / \alpha 2 \delta$ channels (Fig. $3 D$, shaded lines). Neither of them affected the inactivation of $\alpha 1 \mathrm{C} / \mathrm{HCN} 2 / \alpha 2 \delta$ channels. Compared with its dominant effect in $\alpha 1 \mathrm{C} / \mathrm{HCN} 2$ channel inactivation (Fig. 3, $A$ and $B$ ), CaM plays a minor role in $\alpha 1 \mathrm{C} / \mathrm{HCN} 2 / \alpha 2 \delta$ channel inactivation (Figs. $3, C$ and $D$ ), indicating a potential role of $\alpha 2 \delta$-subunit in the $\alpha 1 \mathrm{C} / \mathrm{HCN} 2$ channel inactivation.

\section{a1C/HCN2 channel interaction requires $\mathrm{NH}_{2}$ terminus of $\mathrm{HCN} 2$ and IQ motif of $\alpha 1 \mathrm{C}$}

The fact that the fast inactivation of $\alpha 1 \mathrm{C} / \mathrm{HCN} 2$ is $\mathrm{Ca}^{2+}$ independent (since we used $\mathrm{Ba}^{2+}$ as a charge carrier) suggested that CaM may not be the only mechanism for $\alpha 1 \mathrm{C} / \mathrm{HCN} 2$ channel interaction. To explore an unconventional idea that HCN2 may also interact with 
a1C, we studied the current expression of 1) the wild-type a1C with a HCN2 mutant lacking the $\mathrm{NH}_{2}$ terminus [HCN2(-N)] and 2) the wild-type $\mathrm{HCN} 2$ with an $\alpha 1 \mathrm{C}$ mutant lacking the IQ motif in the $\mathrm{COOH}$ terminus [ $\alpha 1 \mathrm{C} \Delta(1623-1666)]$ (Gao et al., 2000). Figure $4 A$ shows that the inactivation of $\alpha 1 \mathrm{C} / \mathrm{HCN} 2$ (solid line) was eliminated by either HCN2(N) or $\alpha 1 C \Delta(1623-1666)$ (shaded lines).

We next examined how $\alpha 1 \mathrm{C}$ and $\mathrm{HCN} 2$ channel mutants interact in the presence of the $\alpha 2 \delta$ subunit. Figure $4 B$ shows that compared with the $\alpha 1 \mathrm{C} / \mathrm{HCN} 2 / \alpha 2 \delta$ channel inactivation (solid line), both $\alpha 1 \mathrm{C} / \mathrm{HCN} 2(-\mathrm{N}) / \alpha 2 \delta$ and $\alpha 1 \mathrm{C} \Delta(1623-1666) / \mathrm{HCN} 2 / \alpha 2 \delta$ channels dramatically slowed the inactivation process (shaded lines). We also observed biphasic inactivation of $\alpha 1 \mathrm{C} / \mathrm{HCN} 2(-\mathrm{N}) / \alpha 2 \delta$ (Fig. $4 B$, shaded line) with its first fast inactivation phase close to the wild type but with a second phase almost linear that cannot be fitted by an exponential function.

We finally studied the effects of $\mathrm{HCN} 2$ and $\alpha 1 \mathrm{C}$ mutants on $\alpha 1 \mathrm{C} / \mathrm{HCN} 2$ inactivation in the presence of both $\alpha 2 \delta$ - and $\beta 1$-subunits. Compared with the wild-type $\alpha 1 \mathrm{C} / \alpha 2 \delta / \beta 1 / \mathrm{HCN} 2$ channel (solid line), the significant slowing of $\alpha 1 \mathrm{C} / \mathrm{HCN} 2$ channel inactivation by either $\mathrm{HCN} 2(-\mathrm{N})$ or $\alpha 1 \mathrm{C} \Delta(1623-1666)$ (shaded lines) still persisted in the presence of $\alpha 2 \delta$ - and $\beta 1$-subunits (Fig. $4 C$ ). At $+10 \mathrm{mV}$, the time constants of $I_{\mathrm{Ba}}$ inactivation were $72.5 \pm 5.6 \mathrm{~ms}$ for $\alpha 1 \mathrm{C} / \alpha 2 \delta / \beta 1 / \mathrm{HCN} 2,108 \pm 13 \mathrm{~ms}$ for $\alpha 1 \mathrm{C} / \alpha 2 \delta / \beta 1 / \mathrm{HCN} 2(-\mathrm{N})$, and $498 \pm 43 \mathrm{~ms}$ for $\alpha 1 \mathrm{C} \Delta(1623-1666) / \alpha 2 \delta / \beta 1 / \mathrm{HCN} 2$, respectively $(n=5-7)$ (Fig. $4 D$ ). Together, these results suggest that the $\mathrm{NH}_{2}$ terminus of HCN2 and the IQ motif of $\alpha 1 \mathrm{C}$ are the structural elements required for the inactivation of the $\alpha 1 \mathrm{C} / \mathrm{HCN} 2$ channel. 


\section{Relative roles of $\mathrm{CaM}, \alpha 2 \delta$, and $\beta 1$ in the inactivation of $\alpha 1 \mathrm{C} / \mathrm{HCN} 2$ channels}

To examine the relative contribution of $\alpha 2 \delta$ and $\mathrm{CaM}$ to the inactivation of $\alpha 1 \mathrm{C} / \mathrm{HCN} 2$ channels, we compared the inactivation time constants for different combinations of $\alpha 1 \mathrm{C}$, $\mathrm{HCN} 2, \alpha 2 \delta$, and CaM, including their respective mutants, except for $\alpha 1 \mathrm{C} 1905 \mathrm{x} / \mathrm{HCN} 2$. a1C 1905x is a distal $\mathrm{COOH}$-terminal truncated form of $\alpha 1 \mathrm{C}$, which is used as a control for $\alpha 1 \mathrm{C} \Delta(1623-1666)$ function (Gao et al., 2000). We found that $\alpha 1 \mathrm{C} \Delta(1623-1666)$ and HCN2(-N) are the two most significant structural motifs that contribute to the inactivation of $\alpha 1 \mathrm{C} / \mathrm{HCN} 2$ channels independently of $\alpha 2 \delta$ (Fig. $4 D$ ). We also found, surprisingly, that overexpression of $\mathrm{CaM}_{(\mathrm{WT})}$ did not accelerate (as one would expect), but slowed, the inactivation of $\alpha 1 \mathrm{C} / \mathrm{HCN} 2 / \alpha 2 \delta$ or $\alpha 1 \mathrm{C} 1905 \mathrm{x} / \mathrm{HCN} 2 / \alpha 2 \delta$ channels (Fig. $4 D$ ).

In the absence of $\alpha 2 \delta$, there was a distinct difference between CDI and VDI for $\alpha 1 \mathrm{C} / \mathrm{HCN} 2$ channels (Fig. $2 B$ ). In the presence of $\alpha 2 \delta$, however, the difference between CDI and VDI disappeared (Fig. 4E). Combined with the diminished difference between CDI and VDI for $\alpha 1 C / \alpha 2 \delta / \beta 1 / \mathrm{HCN} 2$ (Fig. $1 B$ ), the results provide additional evidence for a major role of $\alpha 2 \delta$ in the inactivation of $\alpha 1 \mathrm{C} / \mathrm{HCN} 2$ channels. In comparing Fig. $4, C$ and $E, \beta 1$ helped complete the $I_{\mathrm{Ba}}$ inactivation of $\alpha 1 \mathrm{C} / \mathrm{HCN} 2 / \alpha 2 \delta$ channels, which are consistent with the results shown in Fig. $1 B$. 


\section{Slowing $\mathrm{Ca}^{2+}$ inactivation by disrupting $\alpha 1 \mathrm{C} / \mathrm{HCN} 2$ association in hippocampus neurons}

$\mathrm{NH}_{2}$ terminus of $\mathrm{HCN} 2$ was reported to be essential in the formation of the homo- and heteromeric channels at the plasma membrane (Proenza et al., 2002). Shown in Fig. 5A, formation of the rat hippocampal neuron network was visualized after 5-6 days in culture. After 24- to 48-h transfection of HCN2(-N) with GFP, the expression of HCN2(N) was indicated by the fluorescent image (Fig. 5B) compared with the brightfield image of the same region (Fig. 5A). The fluorescent neurons were subject to the patch-clamp recordings. Shown in Fig. $5 C$, overexpression of $\mathrm{HCN} 2(-\mathrm{N})$ induced a remarkably slow inactivation component (light shaded line) that was not present in the control neurons or in neurons transfected with the empty vector (solid line, as a transfection control). On the other hand, transfection with the full-length $\mathrm{HCN} 2$ accelerated the $I_{\mathrm{Ba}}$ inactivation (dark shaded line in Fig. 5C). Currents were verified by verapamil $(10 \mu \mathrm{M})$ blockade (Fig. $5 C$ ). At $+10 \mathrm{mV}$, time constants of $I_{\mathrm{Ba}}$ inactivation were $26.5 \pm 2.9 \mathrm{~ms}(n=9)$ for control hippocampal neurons $(\mathrm{HN}), 35.1 \pm 3.5 \mathrm{~ms}(n=6)$ for $\mathrm{HN}+$ vector, $66.1 \pm 3.3 \mathrm{~ms}(n=7)$ for $\mathrm{HN}+\mathrm{HCN} 2(-\mathrm{N})$, and $19.0 \pm 2.2 \mathrm{~ms}(n=10)$ for $\mathrm{HN}+\mathrm{HCN} 2$ (Fig. $5 D)$. The altered time constants for channel inactivation were marked with statistical significance compared with the control $\mathrm{HN}$ or $\mathrm{HN}$ transfected with vector (Fig. $5 D$ ). 
A

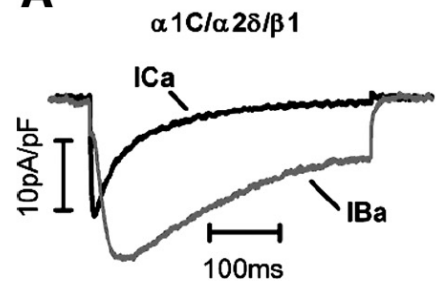

D

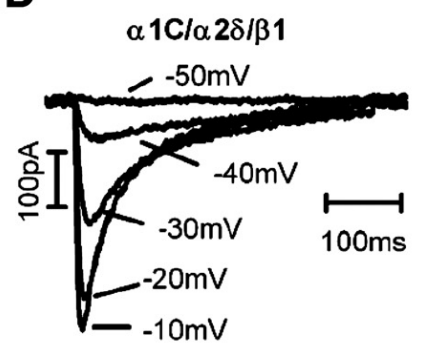

B

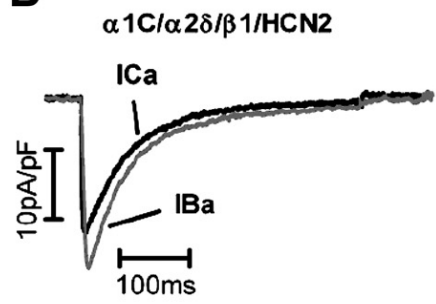

E

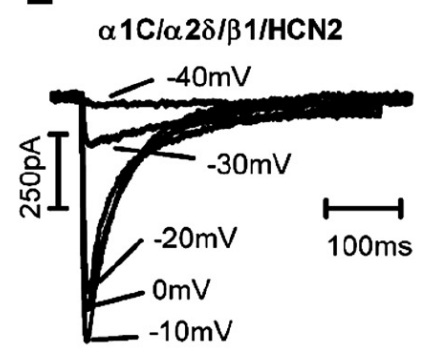

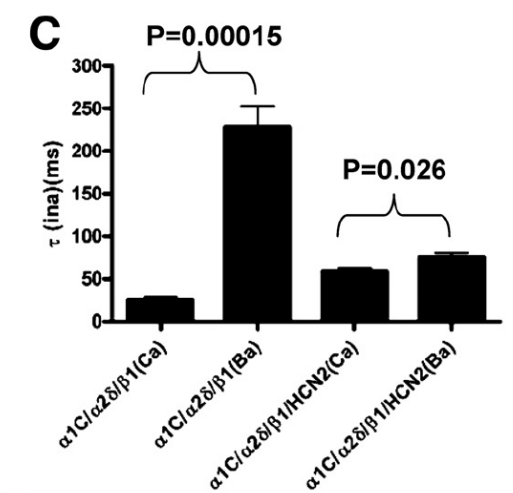

$\mathbf{F}$

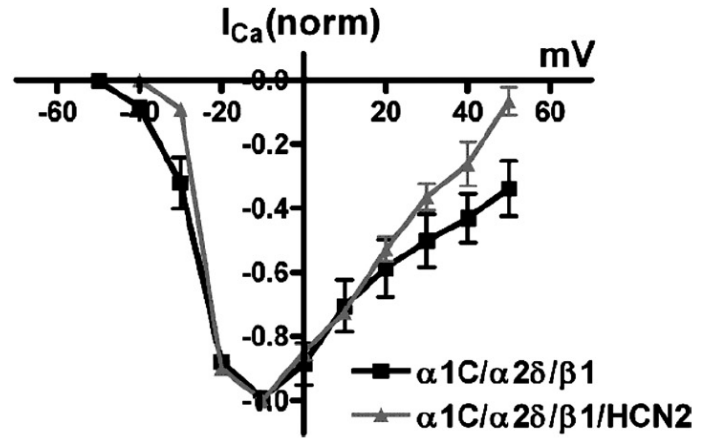

Fig 1. $\mathrm{Ca}^{2+}$-dependent inactivation (CDI) and voltage-dependent inactivation (VDI) of $\alpha 1 C / \alpha 2 \delta / \beta 1$ and $\alpha 1 C / \alpha 2 \delta / \beta 1 / H C N 2$ in HEK-293. $A$ : $\mathrm{Ca}^{2+}$ current $\left(I_{\mathrm{Ca}}\right.$; solid line) and $\mathrm{Ba}^{2+}$ current $\left(I_{\mathrm{Ba}}\right.$; shaded line) of $\alpha 1 \mathrm{C} / \alpha 2 \delta / \beta 1$ recorded at $+10 \mathrm{mV} . B: I_{\mathrm{Ca}}$ (solid line) and $I_{\mathrm{Ba}}$ (shaded line) of $\alpha 1 \mathrm{C} / \alpha 2 \delta / \beta 1 / \mathrm{HCN} 2$ recorded at $+10 \mathrm{mV}$. $C$ : time constants $(\tau)$ for inactivation of $I_{\mathrm{Ca}}$ and $I_{\mathrm{Ba}}$ at $+10 \mathrm{mV}$ for $\alpha 1 \mathrm{C} / \alpha 2 \delta / \beta 1$ and $\alpha 1 \mathrm{C} / \alpha 2 \delta / \beta 1 / \mathrm{HCN} 2$, respectively. For simplicity of comparison, we best fit the currents with one exponential function. $P$ values shown are the statistical results of comparing $I_{\mathrm{Ca}}$ and $I_{\mathrm{Ba}}$ inactivation. $D$ and $E: I_{\mathrm{Ca}}$ currents recorded in the test pulses ranging from -50 to $+50 \mathrm{mV}$ in $10-\mathrm{mV}$ increments for $\alpha 1 \mathrm{C} / \alpha 2 \delta / \beta 1(D)$ and $\alpha 1 \mathrm{C} / \alpha 2 \delta / \beta 1 / \mathrm{HCN} 2(E)$, respectively. A 400 -ms depolarizing pulse was used. Holding potential was $-90 \mathrm{mV}$. F: current-voltage relationship curves for $\alpha 1 \mathrm{C} / \alpha 2 \delta / \beta 1$ and $\alpha 1 \mathrm{C} / \alpha 2 \delta / \beta 1 / \mathrm{HCN} 2$. 
A
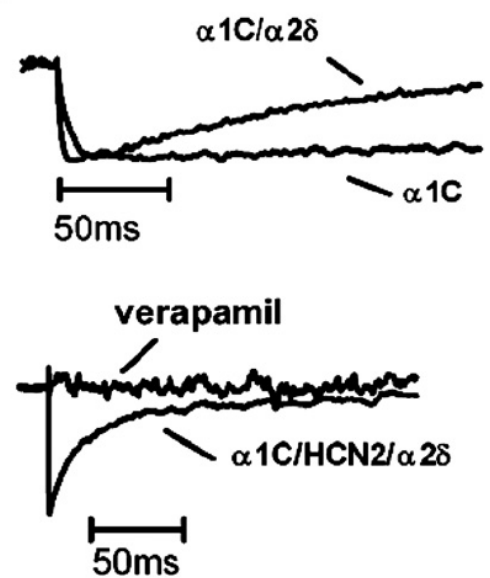

C HCN2 (-125mV)

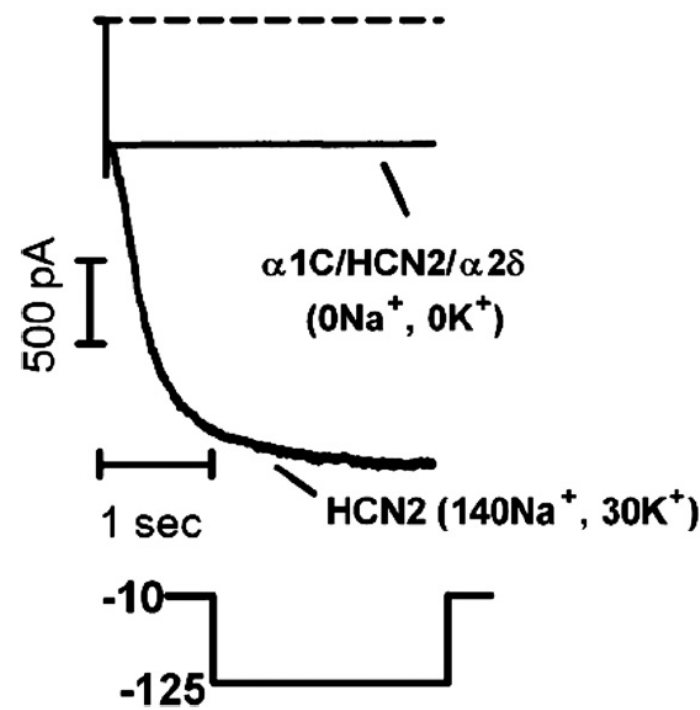

B $\quad \alpha 1$ 1C/HCN2
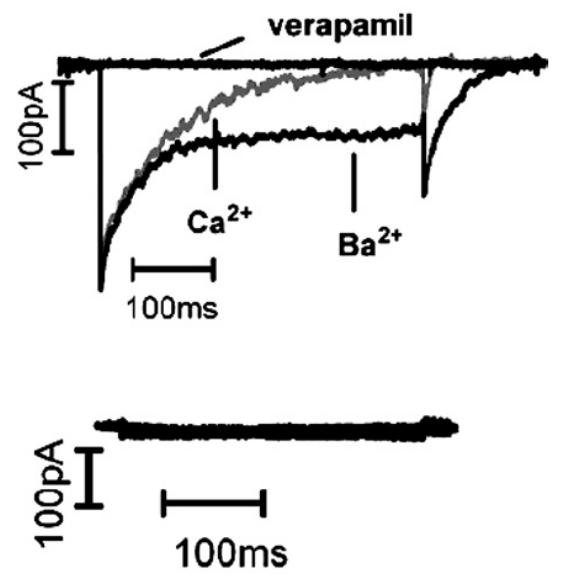

D $" \mathrm{I}_{\mathrm{Ca}} "(+10 \mathrm{mV})$

HCN2
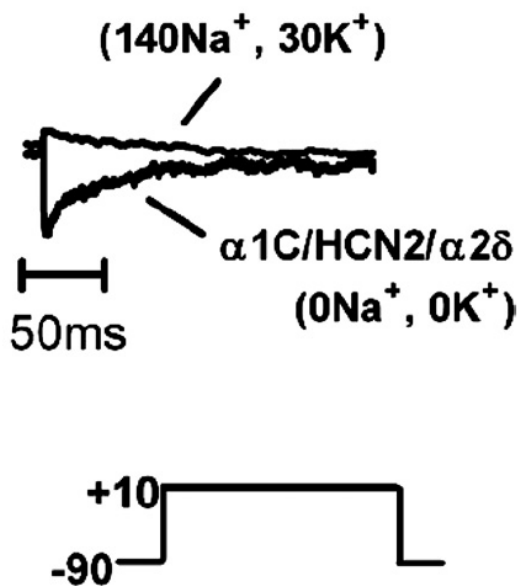

Fig 2. $\alpha 1 \mathrm{C} / \mathrm{HCN} 2$ channel inactivation in HEK-293 cells. $A$, top: current expression of $\alpha 1 \mathrm{C}$ and $\alpha 1 \mathrm{C} / \alpha 2 \delta$, respectively. Bottom, current inactivation of $\alpha 1 \mathrm{C} / \mathrm{HCN} 2 / \alpha 2 \delta$ that was blocked by $10 \mu \mathrm{M}$ verapamil. Pulse duration was $200 \mathrm{~ms}$. $B$, top: $\alpha 1 \mathrm{C} / \mathrm{HCN} 2$ inactivation using $2 \mathrm{mM} \mathrm{Ba}^{2+}$ (solid line) or $2 \mathrm{mM} \mathrm{Ca}^{2+}$ (shaded line) as the charge carrier, which was blocked by $10 \mu \mathrm{M}$ verapamil. Pulse duration was $400 \mathrm{~ms}$. Bottom, absence of timedependent inward current expressions in HEK-293 cells without plasmid transfection. Pulse duration was $300 \mathrm{~ms}$. In $A$, the current inactivation was normalized for better 
comparison of the inactivation kinetics. $C$ : hyperpolarizing pulse control experiments. In cells expressing only $\mathrm{HCN} 2, I_{\mathrm{HCN} 2}$ was elicited by a 3 -s hyperpolarizing pulse to -125 $\mathrm{mV}$ from the holding potential of $-10 \mathrm{mV}$ in modified Tyrode solution containing 140 $\mathrm{mM} \mathrm{Na}^{+}$and $30 \mathrm{mM} \mathrm{K}$; in cells coexpressing $\alpha 1 \mathrm{C} / \mathrm{HCN} 2 / \alpha 2 \delta$, the same hyperpolarizing pulse could not detect $I_{\mathrm{HCN} 2}$ in the external and internal solutions used to record $I_{\mathrm{Ba}}$. Although the leak current varies from cell to cell, we chose a leak current that closely aligned with the HCN2 time-dependent inward current for better comparison. The pulse protocol is shown in the inset. The dashed line shows the holding current. $D$ : depolarizing pulse control experiments. In solutions without $\mathrm{Na}^{+}$and $\mathrm{K}^{+}$ions $\left(0 \mathrm{Na}^{+}, 0 \mathrm{~K}^{+}\right), I_{\mathrm{Ba}}$ was recorded only in cells coexpressing $\alpha 1 \mathrm{C} / \mathrm{HCN} 2 / \alpha 2 \delta$, not in cells expressing only $\mathrm{HCN} 2$. The pulse protocol is shown in the inset. 

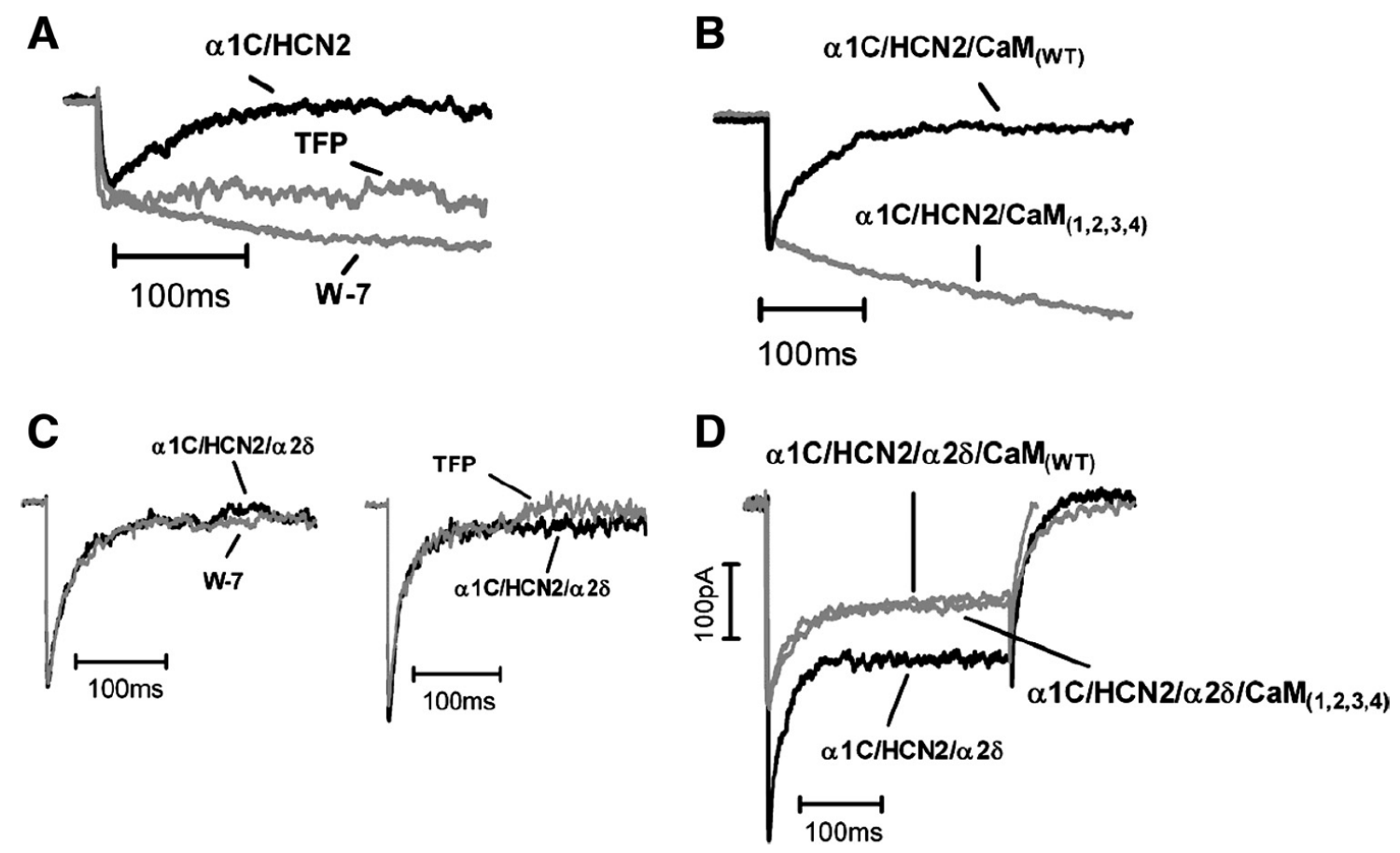

Fig 3. Role of calmodulin (CaM) in $\alpha 1 \mathrm{C} / \mathrm{HCN} 2$ channel inactivation. $A: \alpha 1 \mathrm{C} / \mathrm{HCN} 2$ channel inactivation (solid line) and lost inactivation in the presence of the CaM inhibitor W-7 or trifluoperazine (TFP) (shaded lines). $B: \alpha 1 \mathrm{C} / \mathrm{HCN} 2 / \mathrm{CaM}_{(\mathrm{WT})}$ channel inactivation (solid line) and lost inactivation of $\alpha 1 \mathrm{C} / \mathrm{HCN} 2 / \mathrm{CaM}_{(1,2,3,4)}$ channels (shaded line). CaM(WT), wild-type calmodulin; $\mathrm{CaM}_{(1,2,3,4)}$, a dominant negative calmodulin mutant. $C$ : $\alpha 1 \mathrm{C} / \mathrm{HCN} 2 / \alpha 2 \delta$ channel inactivation in the absence (solid line) and presence of $\mathrm{W}-7$ (shaded line; left) or TFP (shaded line; right). $D: \alpha 1 \mathrm{C} / \mathrm{HCN} 2 / \alpha 2 \delta$ channel inactivation in the absence (solid line) and presence of $\mathrm{CaM}_{(1,2,3,4)}$ ( shaded line). 

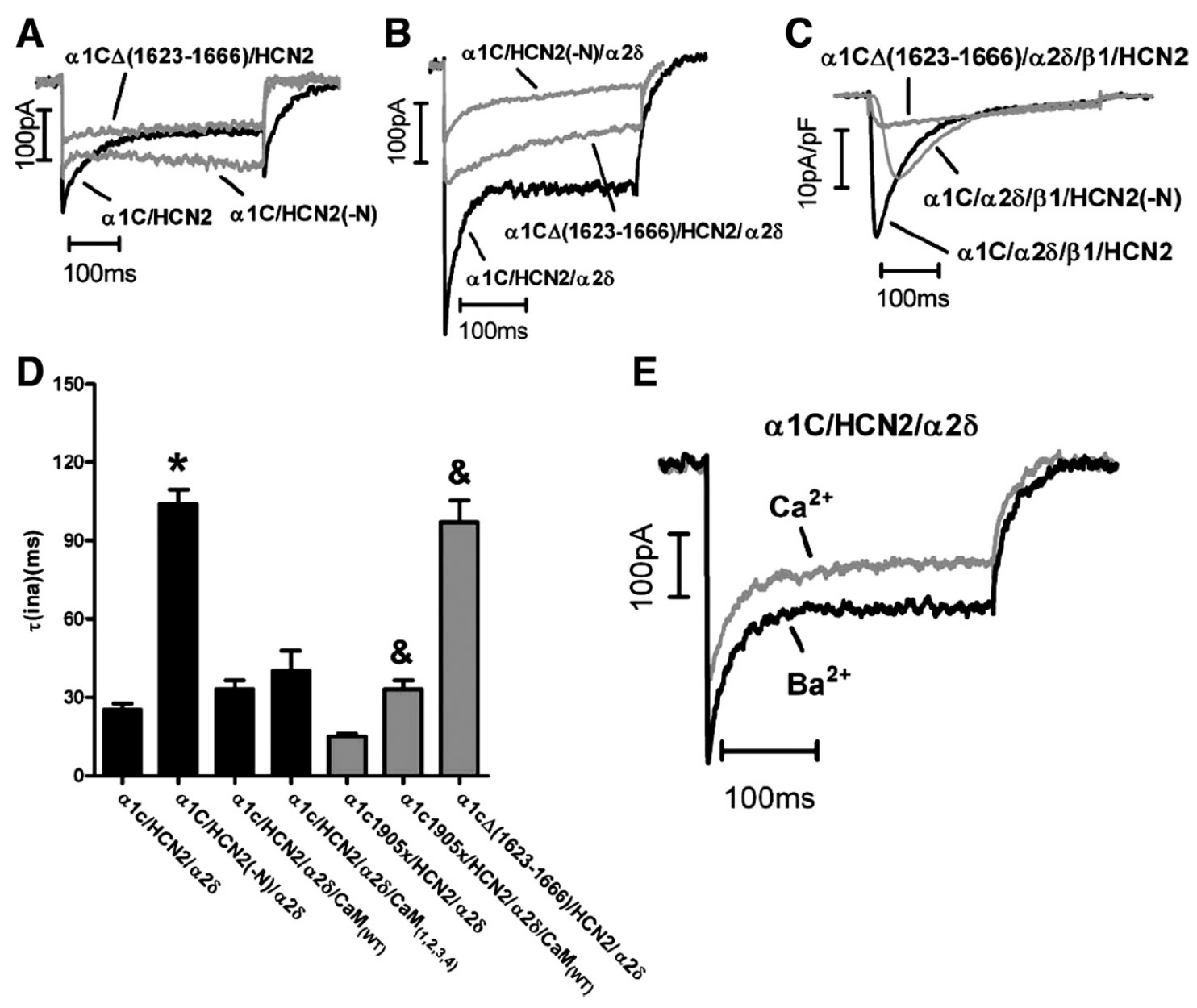

Fig 4. $\alpha 1 \mathrm{C} / \mathrm{HCN} 2$ interaction requires $\mathrm{NH}_{2}$ terminus of $\mathrm{HCN} 2$ and IQ motif of $\alpha 1 \mathrm{C}$. A: $\alpha 1 \mathrm{C} / \mathrm{HCN} 2$ channel inactivation for wild-type (solid line), $\alpha 1 \mathrm{C} \Delta(1623-1666) / \mathrm{HCN} 2$ (shaded line), and $\alpha 1 \mathrm{C} / \mathrm{HCN} 2(-\mathrm{N})$ channels (shaded line). $B: \alpha 1 \mathrm{C} / \mathrm{HCN} 2 / \alpha 2 \delta$ channel inactivation for wild-type (solid line), $\alpha 1 \mathrm{C} \Delta(1623-1666) / \mathrm{HCN} 2 / \alpha 2 \delta$ (shaded line), and $\alpha 1 \mathrm{C} / \mathrm{HCN} 2(-\mathrm{N}) / \alpha 2 \delta$ channels (shaded line). $C$ : inactivation of $\alpha 1 \mathrm{C} / \alpha 2 \delta / \beta 1 / \mathrm{HCN} 2$ (solid line), $\alpha 1 \mathrm{C} \Delta(1623-1666) / \alpha 2 \delta / \beta 1 / \mathrm{HCN} 2$ (shaded line), and $\alpha 1 \mathrm{C} / \alpha 2 \delta / \beta 1 / \mathrm{HCN} 2(-\mathrm{N})$ (shaded line). $D$ : inactivation time constants measured at $+10 \mathrm{mV}$ for showing the significant role of $\mathrm{HCN} 2(-\mathrm{N})\left[\alpha 1 \mathrm{C} / \mathrm{HCN} 2 / \alpha 2 \delta, \quad \alpha 1 \mathrm{C} / \mathrm{HCN} 2(-\mathrm{N}) / \alpha 2 \delta, \alpha 1 \mathrm{C} / \mathrm{HCN} 2 / \alpha 2 \delta / \mathrm{CaM}_{(\mathrm{WT})}\right.$, and $\alpha 1 \mathrm{C} / \mathrm{HCN} 2 / \alpha 2 \delta / \mathrm{CaM}_{(1,2,3,4)}$; solid bars] and the significant role of $\alpha 1 \mathrm{C} \Delta(1623-1666)$ 
$\alpha 1 \mathrm{C} 1905 \mathrm{x} / \mathrm{HCN} 2, \quad \alpha 1 \mathrm{C} 1905 \mathrm{x} / \mathrm{HCN} 2 / \alpha 2 \delta, \quad \alpha 1 \mathrm{C} 1905 \mathrm{x} / \mathrm{HCN} 2 / \alpha 2 \delta / \mathrm{CaM}_{(\mathrm{WT})}, \quad$ and $\alpha 1 \mathrm{C} \Delta(1623-1666) / \mathrm{HCN} 2 / \alpha 2 \delta$; shaded bars]. Results are expressed as means \pm se. ${ }^{*} P<$ 0.0001 , statistically significant difference compared with $\alpha 1 \mathrm{C} / \mathrm{HCN} 2 / \alpha 2 \delta ; P=0.07$ for $\alpha 1 \mathrm{C} / \mathrm{HCN} 2 / \alpha 2 \delta / \mathrm{CaM}_{(\mathrm{WT})}$ and $P=0.08$ for $\alpha 1 \mathrm{C} / \mathrm{HCN} 2 / \alpha 2 \delta / \mathrm{CaM}_{(1,2,3,4)}(n=6-12) . \& P<$ 0.0001 , statistically significant difference compared with $\alpha 1 \mathrm{C} / \mathrm{HCN} 2 / \alpha 2 \delta$ or $\alpha 1 \mathrm{C} 1905 \mathrm{x} / \mathrm{HCN} 2 / \alpha 2 \delta ; P=0.006$ for $\alpha 1 \mathrm{C} 1905 \mathrm{x} / \mathrm{HCN} 2 / \alpha 2 \delta / \mathrm{CaM}_{(\mathrm{WT})}$ and $P=0.0003$ for $\alpha 1 \mathrm{C} \Delta(1623-1666) / \mathrm{HCN} 2 / \alpha 2 \delta(n=5-12)$. E: inactivation of $\alpha 1 \mathrm{C} / \mathrm{HCN} 2 / \alpha 2 \delta$ channel in the presence of $\mathrm{Ba}^{2+}$ (solid line) and $\mathrm{Ca}^{2+}$ (shaded line), respectively. 

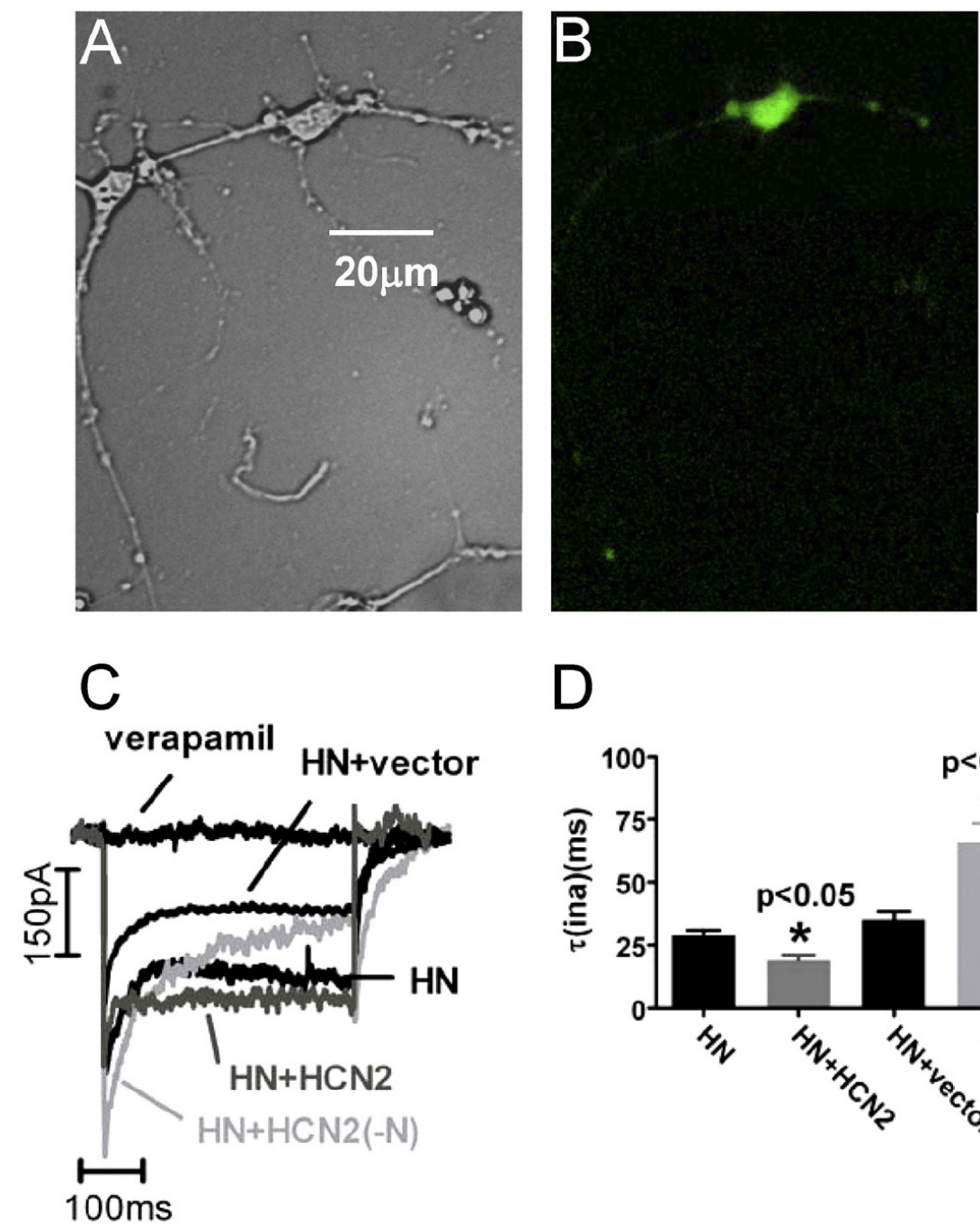

D

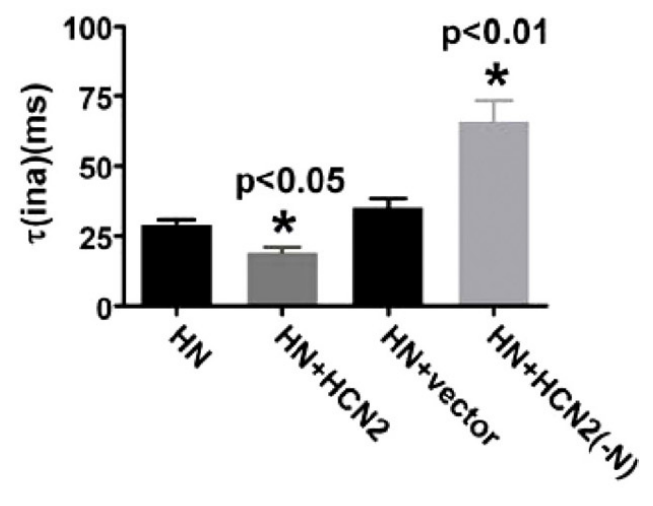

Fig 5. Altered L-type $\mathrm{Ca}^{2+}$ channel (LTCC) inactivation in rat hippocampal neurons (HN) overexpressing HCN2(-N) and the full-length HCN2. A brightfield image $(A)$ and fluorescence image $(B)$ of the region is shown $48 \mathrm{~h}$ posttransfection of $\mathrm{HCN} 2(-\mathrm{N})$ or $\mathrm{HCN} 2$ with GFP. $C: \mathrm{Ca}^{2+}$ current inactivation using $\mathrm{Ba}^{2+}$ as a charge carrier in a control neuron and in neurons transfected with the empty vector $(\mathrm{HN}+$ vector), in a neuron transfected with $\mathrm{HCN} 2(-\mathrm{N})[\mathrm{HN}+\mathrm{HCN} 2(-\mathrm{N})]$, and in a neuron transfected with $\mathrm{HCN} 2$ $(\mathrm{HN}+\mathrm{HCN} 2) . I_{\mathrm{Ba}}$ currents were recorded at $+10 \mathrm{mV}$. The holding potential was at -90 $\mathrm{mV}$. Pulse duration was $400 \mathrm{~ms}$. P4 protocol was used. The $\mathrm{HN}+$ vector trace was 
moved up by $60 \mathrm{pA}$ for clear illustration of currents under different conditions. $D$ : inactivation time constants in $\mathrm{HN}, \mathrm{HN}$ transfected with $\mathrm{HCN} 2, \mathrm{HN}$ transfected with the vector, and $\mathrm{HN}$ transfected with $\mathrm{HCN} 2(-\mathrm{N})$. Results are expressed as means \pm se. $P$ values indicate statistical significance compared with either $\mathrm{HN}$ or $\mathrm{HN}+$ vector group. 


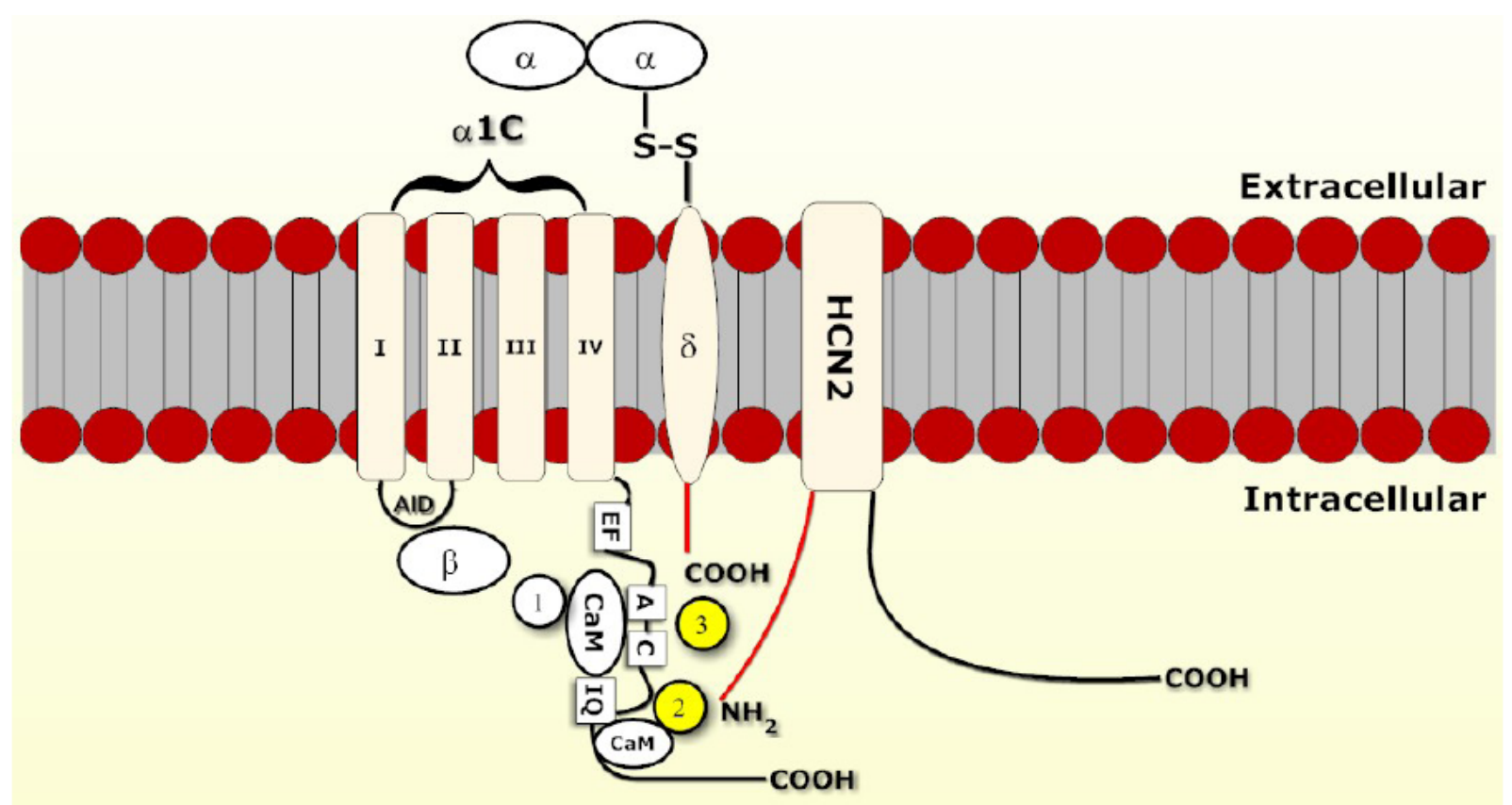

Fig 6. A schematic model depicting the potential roles of HCN2 and $\delta$-subunit in the inactivation of LTCC. Mechanism 1 summarizes the mechanisms for CDI and VDI of LTCC. Mechanism 2 proposes a potential CaM-mediated interaction between the $\mathrm{NH}_{2}$ terminus of HCN2 and the IQ motif of $\alpha 1 \mathrm{C}$. Mechanism 3 proposes a potential interaction between the $\mathrm{COOH}$ tail of the $\delta$-subunit and the $\mathrm{NH}_{2}$ terminus of $\mathrm{HCN} 2$ within the $\mathrm{COOH}$-terminal inactivation machinery of $\alpha 1 \mathrm{C}$-subunit. AID, $\alpha 1$-interaction domain. 


\section{Discussion}

Both $\alpha 1 \mathrm{C}$ - and $\delta$-subunits are transmembrane proteins with $\mathrm{NH}_{2}$ and $\mathrm{COOH}$ tails of $\alpha 1 \mathrm{C}$ and $\mathrm{COOH}$ tail of $\delta$ located intracellularly. $\mathrm{CaM}$ and $\beta 1$-subunit are intracellular proteins attached to the $\alpha 1 \mathrm{C}$ near the inner surface of the membrane. These four proteins constitute the inactivation machinery for the fine control of $\mathrm{Ca}^{2+}$ influx through LTCC in both VDI and CDI (Erickson et al., 2003; Kim et al., 2004; Peterson et al., 1999; Xia et al., 1998).

Our previous studies showed possible involvement of HCN in the modulation of myocyte action potential duration ( $\mathrm{Yu}$ et al., 2007). In the present work, we evaluated the hypothesis that inactivation of LTCC can be modulated via interaction with HCN channels under the experimental conditions that prevent HCN2 channel opening. We showed here that $\mathrm{HCN} 2$ can act as a nonchannel regulatory protein to induce a fast inactivation of $\alpha 1 \mathrm{C}$. To further investigate the possible associated mechanisms involved in the interaction between $\alpha 1 \mathrm{C}$ and $\mathrm{HCN} 2$ channel proteins, we proposed a model shown in Fig 6. Mechanism 1 represents our current understanding of interactions among $\beta$ subunits, which are bound to the $\alpha 1$-interaction domain (AID), apoCaM, and inactivation motifs in the COOH-terminal region of $\alpha 1 \mathrm{C}$. The majority of this conceptual understanding was provided from studies in either Xenopus oocytes or HEK-293 cells coexpressing $\alpha 1 \mathrm{C}$ with $\beta 1$ - and $\alpha 2 \delta$-subunits (Erickson et al., 2003; Peterson et al., 1999, Pitt et al., 2001; Wang et al., 2007; Zuhlke et al., 1998; 1999). Our present study revealed several new concepts.

First, in the absence of $\beta 1$ - and $\alpha 2 \delta$-subunits, HCN2 can induce a faster inactivation of

$\alpha 1 \mathrm{C}$ than $\alpha 2 \delta$ as measured by $I_{\mathrm{Ba}}(\operatorname{compare}$ Fig $2, A$ and $B$ ). This interaction of HCN2 and 
a1C requires CaM (mechanism 2 in Fig 6), since two CaM inhibitors (W-7 and TFP) and the dominant negative $\mathrm{CaM}_{(1,2,3,4)}$ all eliminated the inactivation of $\alpha 1 \mathrm{C} / \mathrm{HCN} 2$ channel (Fig 3, $A$ and $B$ ).

Second, in the presence of the $\alpha 2 \delta$-subunit, $\mathrm{HCN} 2$ can still induce fast inactivation of $\alpha 1 \mathrm{C}$. However, the impact of $\mathrm{CaM}$ in affecting the inactivation of $\alpha 1 \mathrm{C} / \mathrm{HCN} 2 / \alpha 2 \delta$ channel is diminished compared with that for $\alpha 1 \mathrm{C} / \mathrm{HCN} 2$ channel, suggesting a prevalent role of the $\alpha 2 \delta$-subunit in $\alpha 1 \mathrm{C}$ and $\mathrm{HCN} 2$ interaction (mechanism 3 in Fig 6).

Third, mutants of $\mathrm{HCN} 2$ (with the $\mathrm{NH}_{2}$ terminus deleted) and $\alpha 1 \mathrm{C}$ (without IQ motif) revealed a critical role of these two structural elements in mediating the fast inactivation of $\alpha 1 \mathrm{C} / \mathrm{HCN} 2$ channels (Fig 4). It is worth noting that the short $\mathrm{COOH}$ terminus of the $\delta$ subunit is located in the intracellular space. Analysis of amino acids in the $\mathrm{NH}_{2}$ terminus of HCN2 showed an overall positive charge, which can potentially interact with the negatively charged $\mathrm{COOH}$ terminus of $\delta$-subunit. Although previous studies have suggested a vital role for the $\delta$-subunit in accelerating $\alpha 1 \mathrm{C}$ inactivation (Felix et al., 1997; Yasuda et al., 2004), there is limited mechanistic insight into how this process occurs. Our data suggested that a key contributor to this accelerated inactivation may be a dynamic, coordinated interaction among the $\mathrm{COOH}$ terminus of the $\delta$-subunit, the IQ motif of $\alpha 1 \mathrm{C}$, and the $\mathrm{NH}_{2}$ terminus of $\mathrm{HCN} 2$. Alternatively, it is also possible that $\beta 1$ subunit can interfere with the inactivation of $\alpha 1 \mathrm{C} \Delta(1623-1666) / \mathrm{HCN} 2 / \alpha 2 \delta$ and $\alpha 1 \mathrm{C} / \alpha 2 \delta / \mathrm{HCN} 2(-\mathrm{N})$, as well (Fig $4 C$ ). This may be a logical possibility, since the $\beta 1$ subunit is attached to the linker of I-II domains and has been reported to interact with the IQ motif either directly or possibly through the actions of CaM (Zhang et al., 2005). 
Finally, we have demonstrated that an interaction of $\alpha 1 \mathrm{C}$ and $\mathrm{HCN} 2$ is present in native tissues, as evidenced by association of the two channel proteins in the hippocampus and dramatic slowing of $I_{\mathrm{Ba}}$ in hippocampal neurons overexpressing HCN2(-N) (Fig 5). It should be noted that the currents we recorded in hippocampal neurons are not pure LTCCs, since N- and P/Q-calcium channels are present in hippocampal neurons, and verapamil can also inhibit N- and P/Q-type calcium currents (Dobrev et al., 1999). Nevertheless, the fact that overexpression of $\mathrm{HCN} 2(-\mathrm{N})$ mutant in HNs induced a significant slowing of inactivation supports the hypothesis that dynamic interaction between $\alpha 1 \mathrm{C}$ and $\mathrm{HCN} 2$ exists in the native tissue.

\section{Unusual features of $\alpha 1 \mathrm{C} / \mathrm{HCN} 2$ and $\alpha 1 \mathrm{C} / \mathrm{HCN} 2 / \alpha 2 \delta$ channel inactivation}

Dissecting VDI and CDI often has been carried out by using $\mathrm{Ba}^{2+}$ and $\mathrm{Ca}^{2+}$, respectively (Kim et al., 2004; Peterson et al., 1999; Xia et al., 1998; Zuhlke et al., 1998), yielding intriguing results. Not only is CDI about 6-10 times faster than VDI (Kim et al., 2004; Liang et al., 2003), but CDI also can come back to the closed state whereas VDI cannot. The CDI index, measured by the residual fraction of the currents that remains at the end of the test pulse, is much smaller for $\mathrm{Ca}^{2+}$ than for $\mathrm{Ba}^{2+}$ (Erickson et al., 2003; Kim et al., 2004; Peterson et al., 1999; Zuhlke et al., 1998). Interestingly, the speed of VDI can be increased to that of CDI if the IQ motif is deleted (Zuhlke et al., 1998). Furthermore, a single mutation of I to A or E in the IQ motif could eliminate the difference in CDI index for $\mathrm{Ca}^{2+}$ and $\mathrm{Ba}^{2+}$ (Zuhlke et al., 1999). Recently, using a fluorescence resonance energy transfer (FRET) two-hybrid mapping technique, preassociation of apoCaM and a 73amino acid segment containing IQ motif was found to be critical for the slow VDI 
(Erickson et al., 2003). Disruption of this preassociation significantly accelerated the VDI and removed the difference between CDI and VDI (Erickson et al., 2003).

Both $\alpha 1 \mathrm{C} / \mathrm{HCN} 2$ and $\alpha 1 \mathrm{C} / \mathrm{HCN} 2 / \alpha 2 \delta$ channels have exhibited VDI rates that are faster than that reported following the disruption of preassociation of IQ motif and apoCaM (Erickson et al., 2003). Our data suggest that $\mathrm{HCN} 2$, via its $\mathrm{NH}_{2}$ terminus, can interrupt the preassociation of apoCaM with the IQ motif of $\alpha 1 \mathrm{C}$ and that this may contribute to the accelerated VDI inactivation. The requirement of $\mathrm{CaM}$ for $\alpha 1 \mathrm{C} / \mathrm{HCN} 2$ inactivation in the absence of $\alpha 2 \delta$ subunit is supported by the facts that inhibiting CaM activity using either pharmacological inhibitors or overexpression of $\mathrm{CaM}_{(1,2,3,4)}$ all eliminated the inactivation of $\alpha 1 \mathrm{C} / \mathrm{HCN} 2$ (Fig 3, $A$ and $B$ ). On the other hand, $\alpha 1 \mathrm{C} / \mathrm{HCN} 2 / \alpha 2 \delta$ channel inactivation is largely independent of $\mathrm{CaM}$ (Fig 3,C and $D$ ). One possible scenario for this difference may be that the conformational change of CaM resulted from the interaction between the IQ motif and HCN2 is different from that resulted from the interaction between the IQ motif and $\mathrm{HCN} 2 / \alpha 2 \delta$. The interaction between the IQ motif and $\mathrm{HCN} 2$ may lead CaM to undergo a conformational change that exposes its hydrophobic activation site to the $\mathrm{CaM}$ blockers, whereas the interaction between the IQ motif and $\mathrm{HCN} 2 / \alpha 2 \delta$ may induce a different $\mathrm{CaM}$ conformational change that does not make its blockers accessible to the activation site. This possibility would explain the fast VDI of $\alpha 1 \mathrm{C} / \mathrm{HCN} 2 / \alpha 2 \delta$ channel (solid lines in Fig 3, $C$ and $D$ ), the insensitivity of $\mathrm{W}-7$ and TFP to the $\alpha 1 \mathrm{C} / \mathrm{HCN} 2 / \alpha 2 \delta$ currents (Fig 3C), and the lack of a difference between CDI and VDI for the $\alpha 1 \mathrm{C} / \mathrm{HCN} 2 / \alpha 2 \delta$ channel (Fig $4 E$ ). 


\section{Potential implications of $\alpha 1 \mathrm{C}$ and $\mathrm{HCN} 2$ interaction}

In this study, although we have presented evidence demonstrating the interaction of $\alpha 1 \mathrm{C}$ subunit (Cav1.2) with HCN2, interactions of this type might also occur in other types of $\mathrm{Ca}^{2+}$ channels that undergo $\mathrm{CDI}$, such as Cav2 channels, as a common mechanism. Use of $\mathrm{CaM}$ as the key element for $\mathrm{Ca}^{2+}$ channel inactivation has been reported for Cav1 and Cav2 channels (Liang et al., 2003).

Increased $\mathrm{Ca}^{2+}$ influx is associated with a number of neuronal disorders, including neuropathic pain (Altier et al., 2004). Gabapentin, a GABA analog, is an antiepileptic drug that has been used recently to relieve neuropathic pain and is believed to bind the $\alpha 2 \delta 1$-subunit, leading to decreased $\mathrm{Ca}^{2+}$ influx (Altier et al., 2004; Hendrich et al., 2008). The mechanism of how gabapentin binding of $\alpha 2 \delta 1$-subunit decreased $\mathrm{Ca}^{2+}$ influx remains elusive, since the gabapentin action is acute (Altier et al., 2004). The $\alpha 2 \delta$ subunits have been known to enhance the surface expression of $\alpha 1 \mathrm{C}$ and $\mathrm{Ca}^{2+}$ current amplitude (Felix et al., 1997; Yasuda et al., 2004). A recent study demonstrated that chronic treatment with gabapentin was associated with a reduction in the surface expression of $\alpha 2 \delta 2$ and Cav2.1 subunits (Hendrich et al., 2008). On the other hand, the $\alpha 2 \delta$-subunits also promote inactivation of Cav1.2 ( $\alpha 1 \mathrm{C})$ or Cav2.2 (N-type) (Yasuda et al., 2004), and inhibition of the $\alpha 2 \delta$ subunit should, in principle, slow the $\alpha 1 \mathrm{C}$ inactivation, resulting in an increased $\mathrm{Ca}^{2+}$ influx. The interaction of $\alpha 1 \mathrm{C}$ with $\mathrm{HCN} 2$ may

provide an alternative mechanism to prevent an increase in $\mathrm{Ca}^{2+}$ influx under the conditions in which the $\alpha 2 \delta$-subunit expression is reduced. 


\section{Study limitations}

Although we report for the first time a novel and potentially important mechanism for modulation of $\mathrm{Ca}^{2+}$ current inactivation by $\mathrm{HCN} 2$ channel, there are many unanswered questions. For instance, we noticed that the activation of $I_{\mathrm{CaL}}$ also seems to be altered by HCN2 (e.g., Fig. $1 F$ ). In addition, whether $\mathrm{HCN} 2$ may also modulate other $\mathrm{Ca}^{2+}$ channels that share similar inactivation machinery to $\alpha 1 \mathrm{C}$ is unknown. More importantly, under various physiological and especially pathological conditions, the importance of interaction between HCN2 and LTCC requires further investigation, since the expression levels of HCN2, $\alpha 1 \mathrm{C}$, and $\alpha 2 \delta 1$ may be altered. 


\section{Chapter 4}

\section{Trafficking mechanism of HCN4 and its mutant and the novel role of $\mathrm{HCN} 2$ in modulation of $\mathrm{Ca}^{2+}$ influx}

This chapter contains two parts showing unpublished work. Part 1 aims to understand the trafficking mechanisms of HCN4 as well as how tyrosine phosphorylation may help rescue the defective membrane trafficking of $\mathrm{HCN} 4$ mutants identified in patients. Part II aims to further understand the dynamic interaction between HCN and calcium channels. 


\title{
Part I: Trafficking mechanisms of $\mathrm{HCN} 4$ and its mutants identified in patient
}

\begin{abstract}
$\mathrm{D} 553 \mathrm{~N}$ is a missense mutation in the $\mathrm{C}$-linker of HCN4 channel protein. It was found in a patient with severe cardiac arrhythmias including long QT syndrome, ventricular tachycardia polymorphisms, and bradycardia induced by sinus node dysfunction. Although it has been implicated that the cardiac arrhythmia caused by this mutant was due to its trafficking defect, no analysis reveals the underlying mechanism in the subcellular level. Here, we demonstrated that the trafficking defective mutant HCN4 $\mathrm{D} 553 \mathrm{~N}$ is restricted in the endoplasmic reticulum (ER) and perinuclear region. Two days after transfection D553N with the ER maker KKXX in the HEK293 cells, colocalization of these two proteins could be observed. Further, we employed the membrane surface dye FMI34 to show that D553N cannot colocalize with the membrane dye FMI34. On the contrary, wild type HCN4 channels colocalized with the dye FMI34 on the cell membrane which serves as the control for the comparison. In conclusion, our data further demonstrate the subcellular mechanism that the D553N mutant is retained in the ER, resulting in loss-of-function of this mutant at the cell membrane.
\end{abstract}




\section{Introduction}

HCN4 channels play an important role in the regulation of pacemaker activity of sinoatrial nodes. They are responsible for making the "U" turn of maximal diastolic potential (phase 4) for the next cycle of the action potential in sinoatrial nodes. Previous studies have revealed that the abnormality of HCN4 channels in patients is the cause for cardiac arrhythmias such as long QT syndromes, cardiac tachycardia, and sinoatrial nodes dysfunction. So far, several HCN4 mutants have been found in patients that are correlated with cardiac arrhythmias (Milanesi et al., 2006; Schulze et al., 2003; Ueda et al., 2004). Recently, a patient with the severe cardiac arrhythmias was indicated as HCN4 D553N mutant. The electrocardiogram (ECG) also revealed the relative flat "P" wave accompany with long QT interval $(0.64 \mathrm{~s}$ versus $0.44 \mathrm{~s}$ for the normal people) followed by ventricular tachycardia polymorphisms (Ueda et al., 2004). When the cellular mechanisms were further analyzed, they found that this mutant was the dominant negative mutant, which will dominate the phenotype when coexisting with the wild type HCN4 channels.

By using the electrophysiology and imaging, their results indicated that the membrane expression or current expression can nearly be eliminated or significantly reduced after transfection of D553N alone or cotransfection of D553N with wild type HCN4 in COS7 cells (Ueda et al., 2004). The results strongly indicated that the underlying mechanism contributing to loss-of function of D553N mutant is due to defective membrane trafficking. However, what is the subcellular level that this mutant could be trapped during the trafficking process remains obscure. Here we employed the ER maker KKXX 
to investigate the possible colocalization between D553N mutant and this marker using the live-cell confocal imaging. Interestingly, we found that the D553N mutant can colocalize with KKXX whereas it cannot colocalize with a membrane dye FM1-34 which is exclusively expressed on the cell membrane. The control experiment also revealed the colocalization between FM1-34 and wild type HCN4 channels. Overall, our data uncovered the subcellular localization of HCN4 mutant D553N, which may provide the further subcellular evidence indicating the trafficking defect of this mutant. 


\section{Results}

In Fig 1, we co-transfect the D553N mutant carried with DsRed with ER marker KKXX carried with GFP into HEK293 cells. Two days after, the result showed that D553N mutant with DsRed clustered in the centric region of HEK293 cells in which the colocalization with the ER marker KKXX carried with GFP can be observed under the confocal microscope. As ER is distributed around the nucleus, the localization of D553N represented as red can also be observed to surround the region nearby the nucleus and is matched to the KKXX represented as green.

However, the D553N cannot colocalize with the membrane dye FM1-43 (Audrey et al., 2004) which distributes well on the cell membrane as indicated in Fig 2.

Fig 3 is the positive control showing that the wild type HCN4 distributes on the cell membrane in which the colocalization can be detected under the microscope. This result indicated that the D553N mutant is trapped in the ER and fails to localize in the cell membrane. 


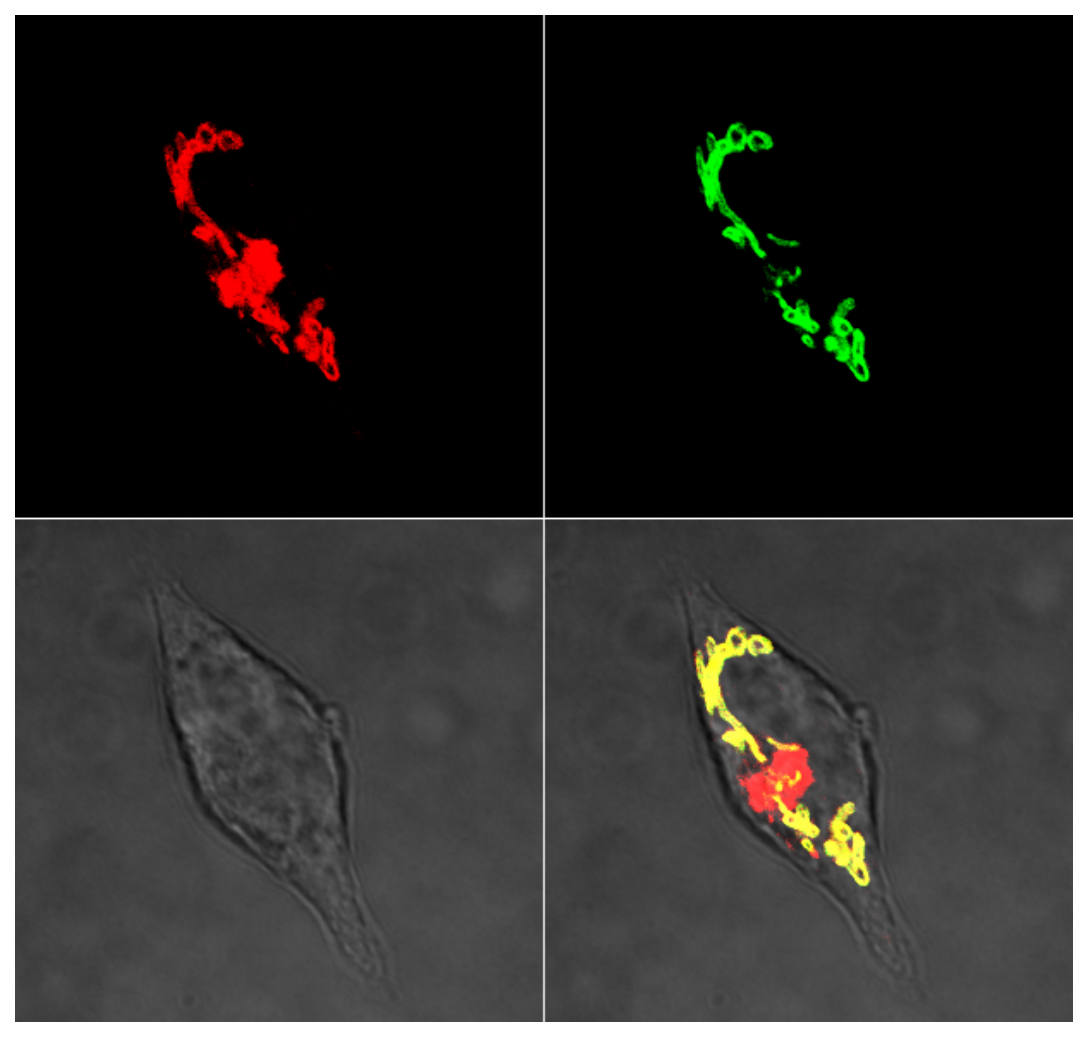

Fig 1. Colocalization between D553N mutant and the ER marker KKXX.

Left up panel shows the D553N carried with DsRed as indicated in the red color. Right up panel represents the expression of the fusion protein KKXX carried with GFP as indicated in green color. Right down panel reveals the colocalization between D553N and KKXX after merge the previous two panels, which indicates as yellow color. Left down panel shows the HEK293 cell in the bright field. 


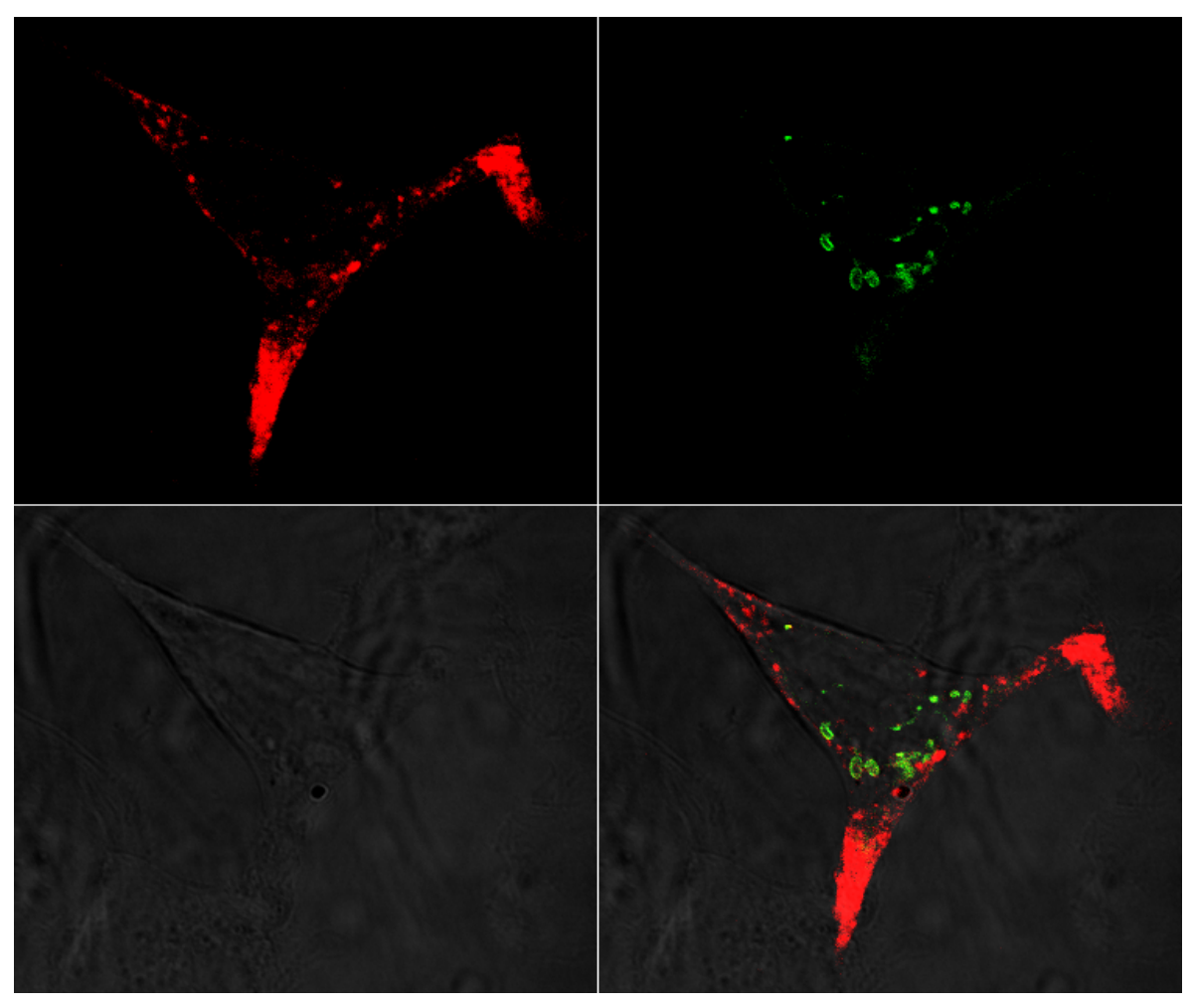

Fig 2. Non-colocalization of wild type HCN4 channels and the ER marker KKXX.

Left up panel shows the subcellular distribution of wild type HCN4 channels, which express throughout the plasma membrane of HEK 293 cells as indicated in red color. Right up panel reveals the expression of KKXX as indicated in green color. Right down panel represents the merged result of the previous two panels. There is no colocalization between the wild type HCN4 channels and the KKXX. Left down panel shows the bright field of the HEK293 cell. 


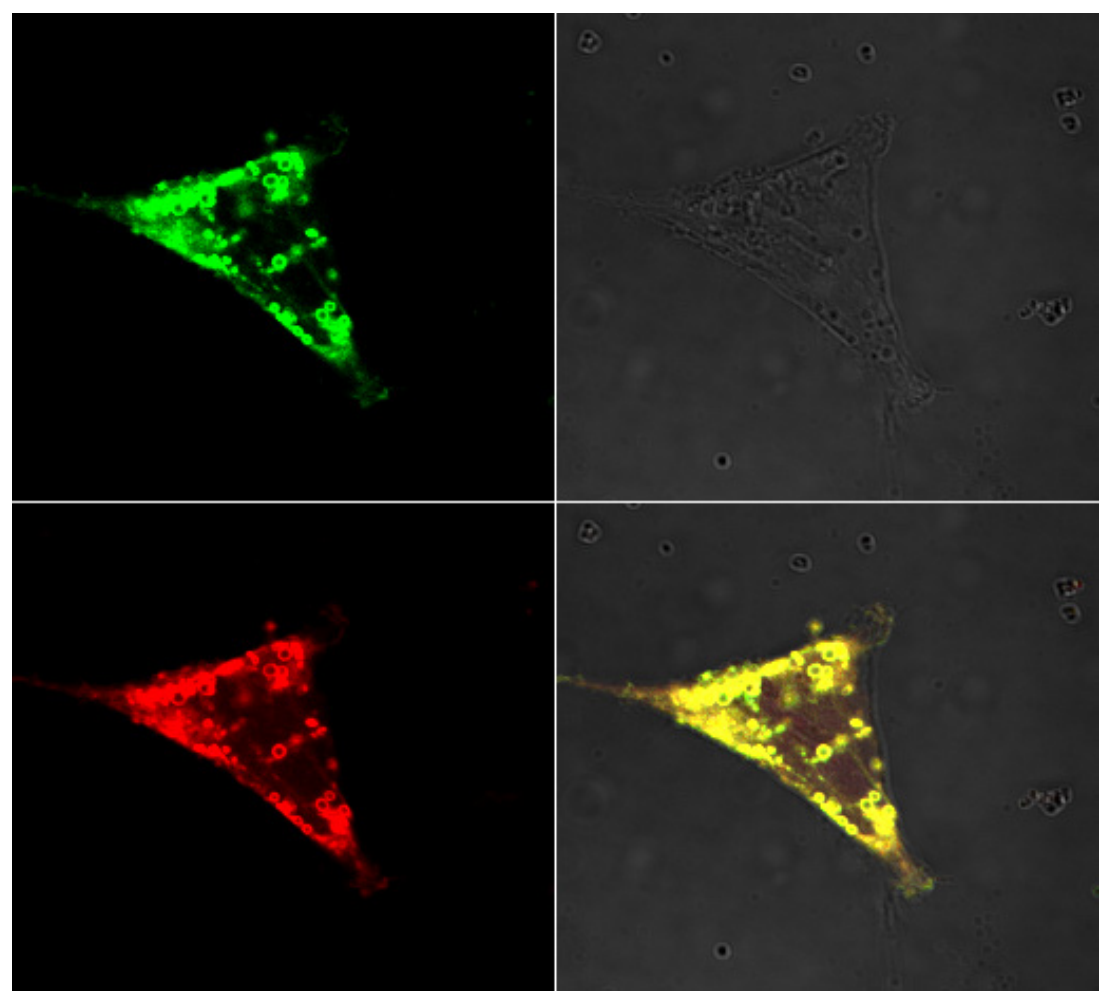

Fig 3. Colocalization between wild type HCN4 channels and the membrane dye FM1-43. Left up panel shows the membrane dye FM1-43 as indicated in the green color. Left down panel represents the distribution of wild type HCN4 channels carried with DsRed as indicated in red color. Right down panel indicates the merged result of the previous two panels and shows the colocalization between the wild type HCN4 channels and FM1-43 as indicated in yellow color. Right up panel shows the whole HEK293 cell in the bright field. 


\section{Discussion}

Our results indicated that the impairment of the membrane expression of single mutation D553N is resulted from trapping in the ER in the process of the trafficking (Fig1-3). These results are consistent with previous reports and may explain why this mutant expresses little current when studied with whole-cell patch clamp.

This membrane trafficking defective D553N mutant has been proposed to cause cardiac arrhythmias such as long QT syndromes accompany with the dysfunction of sinoatrial node (Ueda et al., 2004). As D553N is a single amino acid mutation of HCN4 channels from Asp to Asn, which removes the negative charge, it may thus cause the abnormal folding of the channel protein in the protein synthesis. The disruption of the negative charge may alter the interaction among amino acid residues and results in conformation change of this mutant. Interestingly, ER is a key subcellular organelle for quality control of the channel proteins (Ibba et al., 1999; Wickner et al., 1999). The abnormal folding of D553N mutant may retain the channel in ER, which is subsequently through endosomallysosomal pathway for degradation (Cole et al., 1992; Koh et al., 2005). This may provide us the possible explanation for our results showing the subcellular localization of D553N mutant in ER.

KKXX is the double mutant fusion protein that retains in the ER and serves as an ER marker. We take this advantage and transfect D553N mutant with this marker fusion protein KKXX to study whether they colocalize in the ER in order to elucidate the subcellular localization of D553N. Our results suggest that the trafficking defective 
D553N mutant is attributed to the trapping in the ER and this may make us understand the subcellular mechanism about this mutant induced cardiac arrhythmias. 


\title{
Part II: Association between HCN2 and L-type calcium channels by using fluorescence recovery after photobleaching (FRAP)
}

\begin{abstract}
Calcium influx via L-type calcium channels (LTCCs) plays the critical role in the regulation of intracellular calcium homeostasis. Inactivation of LTCCs is one of the most important mechanisms to modulate calcium influx and prevents the calcium overload induced diseases such as cardiac arrhythmias. Thus, how to delicately regulate the inactivation of LTCCs is directly linked to the intracellular calcium amount and the calcium-based diseases prevention. In the previous studies, using electrophysiology and immunoprecipitation, we have demonstrated that the association between the pore forming subunit $\alpha 1 \mathrm{C}$ of L-type calcium channels (LTCCs) and HCN2 channels can induce the fast inactivation of LTCCs to restrict the calcium influx.
\end{abstract}

Here, we employed another technique (FRAP) to investigate the dynamic association between $\alpha 1 \mathrm{C}$ and HCN2 channels in HEK293 cells. Consistently, the results indicated that the half life $\left(t_{1 / 2}\right)$ of fluorescent recovery was significantly slower in the HEK293 cells co-transfected with $\alpha 1 \mathrm{C}-\mathrm{GFP}$ and $\mathrm{HCN} 2\left(\mathrm{t}_{1 / 2}=14.04 \pm 3.51(\mathrm{~s})\right)$ than those of $\mathrm{N}$ terminal deletion of HCN2 with $\alpha 1 \mathrm{C}-\mathrm{GFP}\left(\mathrm{t}_{1 / 2}=7.83 \pm 2.15(\mathrm{~s})\right)$ or wild type HCN2-GFP

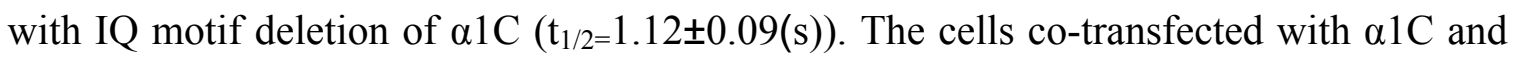
HCN2-GFP also reveals the relatively slower half life $\left(t_{1 / 2}=10 \pm 1.23(\mathrm{~s})\right)$. Our studies strongly demonstrate that HCN2 channels are capable of cross-talking with LTCCs at the cell membrane via the association between N-terminus of HCN2 channels and IQ motif 
of $\alpha 1 \mathrm{C}$ in a dynamic manner. These results may provide us supporting evidence of how HCN2 channels can induce the fast inactivation of LTCCs and regulate the calcium influx. 


\section{Introduction}

Inactivation of L-type calcium channels (LTCCs) plays a pivotal role to delicately regulate calcium influx in cardiomyocytes, which is closely linked to cardiac contractility, and if abnormal, leads to calcium-based diseases such as cardiac arrhythmias. The underlying cellular mechanism of inactivation of LTCCs has been wildly studied. It is generally believed that the interaction among the IQ motif on the Cterminal of $\alpha 1 \mathrm{C}$ (the pore region of LTCCs), calmodulin (CaM), and I-II loop near the pore region is involved in the regulation of inactivation of LTCCs. Based on the different mechanisms, the inactivation of LTCCs can be divided into calcium-dependent fast inactivation (CDI) (using $\mathrm{Ca}^{2+}$ as the charge carrier) as well as the voltage-dependent slow inactivation (VDI) (using $\mathrm{Ba}^{2+}$ as the charge carrier). The CDI is dependent on the influx of calcium ions which can interact with CaM, IQ motif, and I-II loop and induce the fast inactivation by closing the pore region of LTCCs. On the other hand, VDI is not calcium dependent (Cens et al., 2006; Kim et al., 2004; Pitt et al., 2001); it is only responsible for the voltage change on the cell membrane in which the slow inactivation will be induced by the conformation change of the CaM, IQ motif, and I-II loop.

Our previous studies have indicated that the inactivation of LTCCs can be fast induced by another ion channel, $\mathrm{HCN} 2$, on the cell membrane approached by electrophysiology in the HEK293 cells co-transfected HCN2 channels with the pore subunit $\alpha 1 \mathrm{C}$ and the different recombinant accessory $\beta$ subunits of LTCCs (Lin et al., 2010). Electrophysiology and immunoprecipitation results further indicated that the underlying mechanism is via the association between N-terminus of HCN2 and IQ motif of $\alpha 1 \mathrm{C}$ 
subunit of LTCCs. In this study, using FRAP to examine the mobility of the wild type HCN2 or a1C subunit and its mutants tagged with GFP in HEK293 cells, we further confirmed the association between N-terminal of HCN2 and IQ motif of $\alpha 1 \mathrm{C}$ subunit. 


\section{Results}

In order to investigate whether $\alpha 1 \mathrm{C}$ and $\mathrm{HCN} 2$ channels associate with each other, we use the FRAP technique to examine the half-time $\left(t_{1 / 2}\right)$ in the HEK293 cells expressing the different recombinant proteins such as $\alpha 1 \mathrm{C}-\mathrm{GFP}$ and $\mathrm{HCN} 2, \alpha 1 \mathrm{C}$ and HCN2-GFP, Nterminal deletion of HCN2 and $\alpha 1 \mathrm{C}-\mathrm{GFP}$, as well as wild type HCN2-GFP and IQ motif deletion of $\alpha 1 \mathrm{C}$.

During the FRAP experiment, photobleaching with high energy light creates a dark and bleached region within the sample, named region of interest (ROI), which is indicated by the arrow in Fig 1. The neighboring molecules, in this case the GFP-labeled proteins, subsequently diffuse into the ROI and replace the bleached molecules to reach a steady state. How fast this steady state is achieved is measured by the half-time $\left(t_{1 / 2}\right)$, which is correlated with how heavy the GFP-labeled proteins are. For example, if the protein is associated with another protein as a complex, the molecular weight is heavier than the protein by itself; therefore the diffusion rate is slower, resulting in a longer $t_{1 / 2}$. The calculation of $t_{1 / 2}$ is shown in Fig 1.

Fig 2 indicated that the half-time $\left(\mathrm{t}_{1 / 2}\right)$ of $\alpha 1 \mathrm{C}$-GFP and HCN2 reveals as $14.04 \pm 3.51(\mathrm{~s})$ $(n=3)$ after subtracting the background and analyzing by the software Igor. When comparing with N-terminal deletion of HCN2 with $\alpha 1 \mathrm{C}$-GFP (indicated as Fig 3 ) or wild type HCN2-GFP with IQ motif deletion of $\alpha 1 \mathrm{C}$ (indicated as Fig 4), the half-time is significantly slower in cells co-transfected $\alpha 1 \mathrm{C}$-GFP with $\mathrm{HCN} 2\left(\mathrm{t}_{1 / 2}=7.83 \pm 2.15(\mathrm{~s})<\right.$ $\mathrm{t}_{1 / 2}=14.04 \pm 3.51(\mathrm{~s})$ and $\mathrm{t}_{1 / 2}=1.12 \pm 0.09(\mathrm{~s})<\mathrm{t}_{1 / 2}=14.04 \pm 3.51(\mathrm{~s})$, respectively) $(\mathrm{n}=3)$. The 
half-time of the cells co-transfected $\alpha 1 \mathrm{C}$ with HCN2-GFP indicated in Fig5 is equal to $10 \pm 1.23(\mathrm{~s})(\mathrm{n}=3)$, which is still slower than those of mutants.

These results indicated the dynamic association of $\mathrm{HCN} 2$ between $\alpha 1 \mathrm{C}$ possibly through N-terminus of HCN2 and IQ motif of $\alpha 1 C$. 

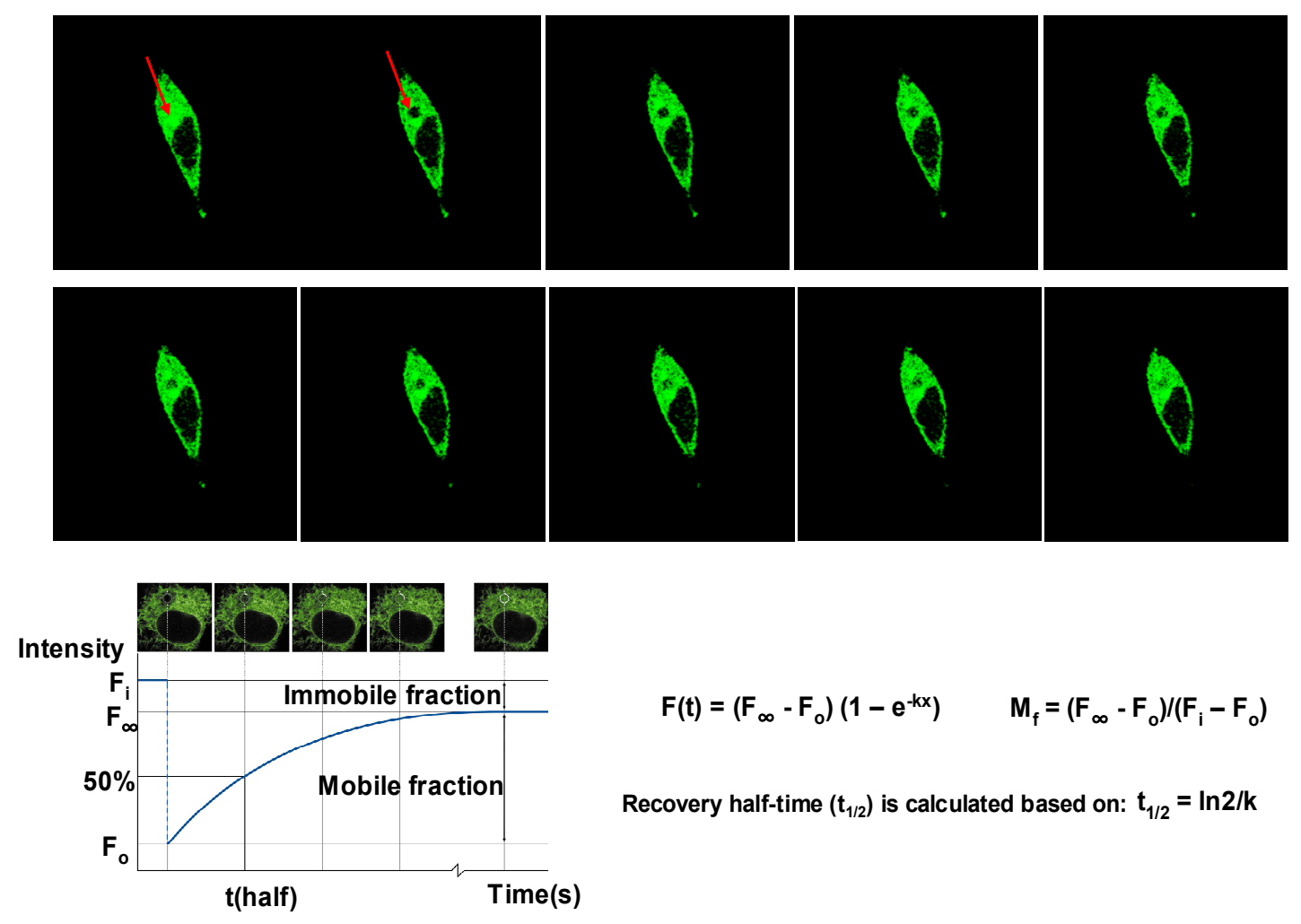

$$
\begin{aligned}
& \qquad F(t)=\left(F_{\infty}-F_{o}\right)\left(1-e^{-k x}\right) \quad M_{f}=\left(F_{\infty}-F_{o}\right) /\left(F_{i}-F_{o}\right) \\
& \text { Recovery half-time }\left(t_{1 / 2}\right) \text { is calculated based on: } t_{1 / 2}=\ln 2 / k
\end{aligned}
$$

\section{Fig 1. Fluorescence recovery after photobleaching (FRAP).}

Red arrows indicate region of interest (ROI) generated by photobleaching. The bleached molecules in ROI are gradually replaced by adjacent molecules (GFP-labeled proteins) and reach the steady state. The half-time $t_{1 / 2}$ represents the time required for the bleach region to recover half of its initial intensity. The calculation of $t_{1 / 2}$ is shown at the bottom. 


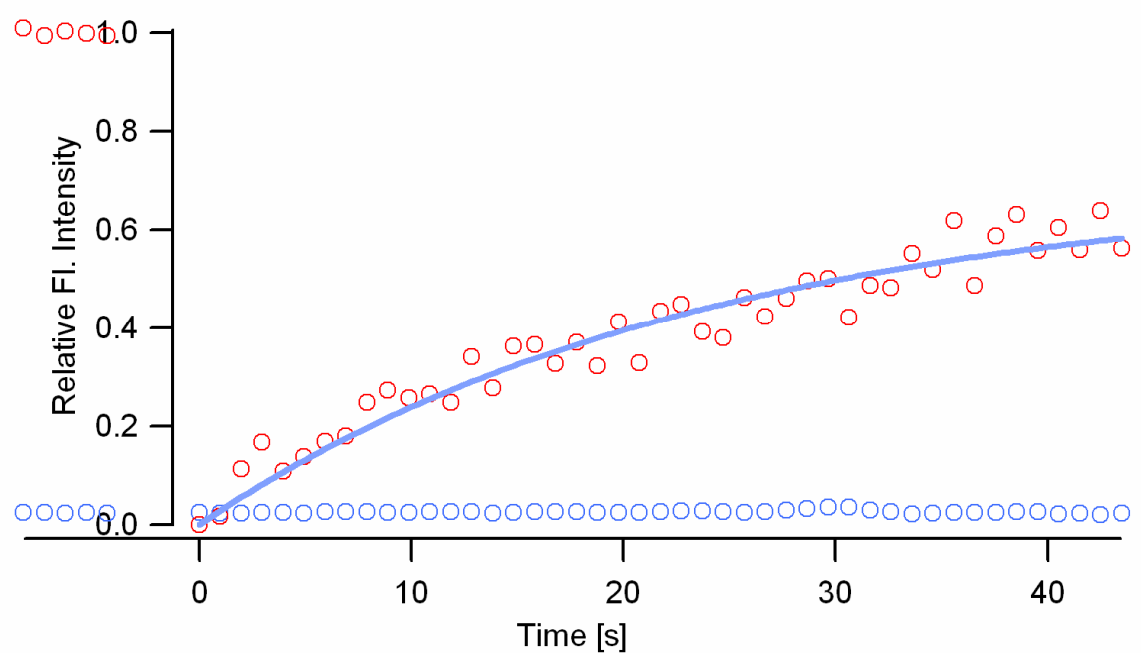

Fig 2. Half-time $\left(\mathrm{t}_{1 / 2}\right)$ of HEK293 cells co-transfected $\alpha 1 \mathrm{C}-\mathrm{GFP}$ with HCN2.

The recovery half-time $\left(\mathrm{t}_{1 / 2}\right)$ of $\alpha 1 \mathrm{C}-\mathrm{GFP}$ and $\mathrm{HCN} 2$ reveals as $14.04 \pm 3.51(\mathrm{~s})$ after subtracting the background and analyzing by the software LM510 and Igor $(n=3)$. Figure 2 indicated the representative figure in HEK293 cells co-transfected HCN2 with $\alpha 1 \mathrm{C}$ tagged with GFP. 


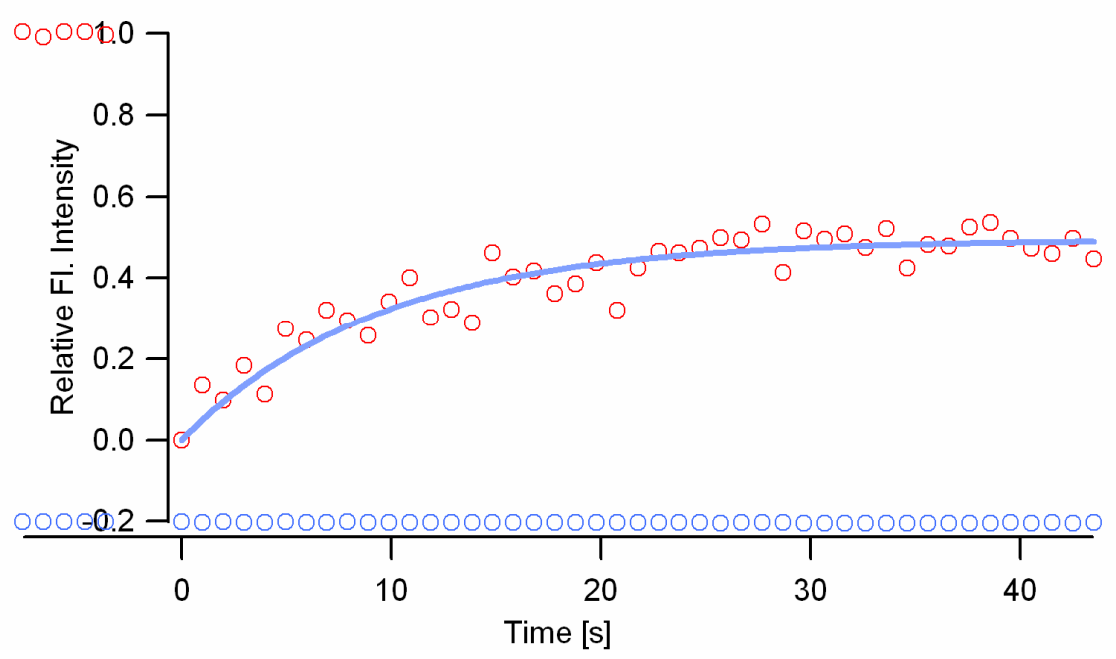

Fig 3. Half-time $\left(t_{1 / 2}\right)$ of HEK293 cells co-transfected $\mathrm{N}$-terminal deletion HCN2 with $\alpha 1$ C-GFP. The recovery half-time is revealed as $7.83 \pm 2.15(\mathrm{~s})$ after analysis by the software LSM510 and Igor (n=3). Figure 3 indicated the representative figure in HEK293 cells co-transfected N-terminal deletion HCN2 with $\alpha 1 \mathrm{C}-\mathrm{GFP}$. 


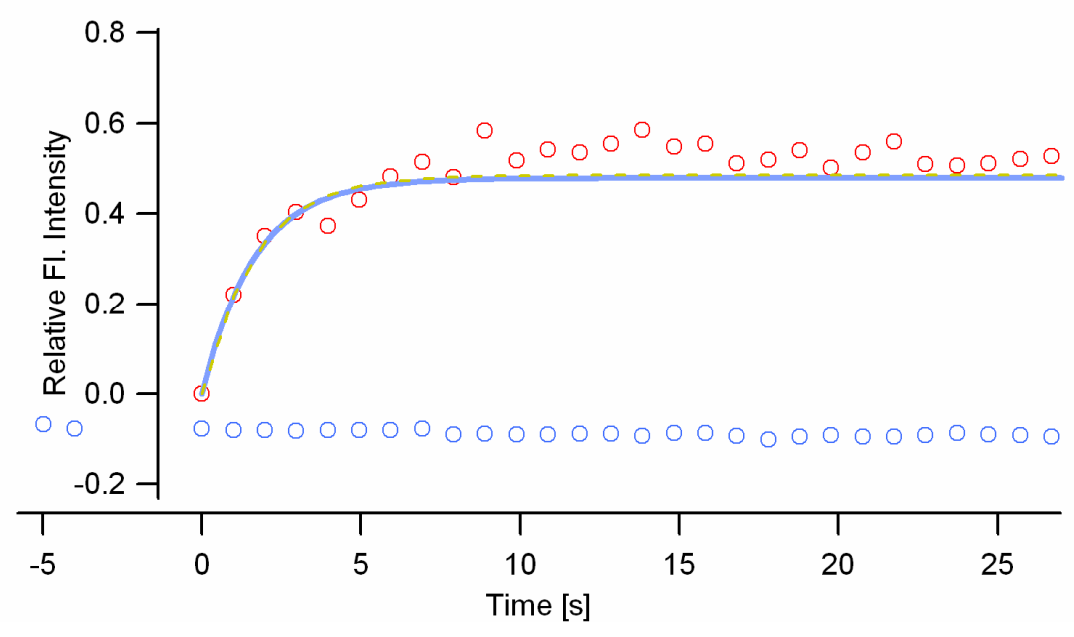

Fig 4. Half-time ( $\left.t_{1 / 2}\right)$ of HEK293 cells co-transfected wild type HCN2-GFP with IQ motif deletion of $\boldsymbol{\alpha 1 C}$. The recovery half-time is revealed as $1.12 \pm 0.09(\mathrm{~s})$ after analysis by the software LSM510 and Igor $(n=3)$. Figure 4 indicated the representative figure in HEK293 cells co-transfected wild type HCN2-GFP with IQ motif deletion of a1C. 


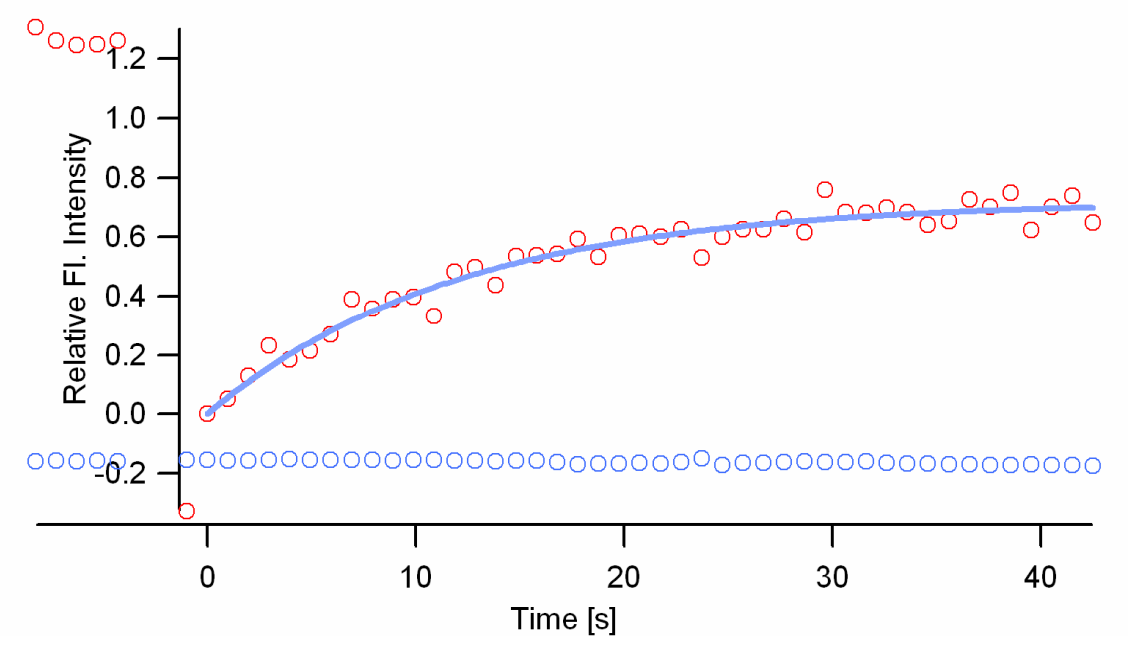

Fig 5. Half-time $\left(t_{1 / 2}\right)$ of HEK293 cells co-transfected $\alpha 1 C$ with HCN2-GFP. The recovery half-time is revealed as $10 \pm 1.23$ (s) after analysis by the software LSM510 and Igor $(n=3)$. Figure 5 indicated the representative figure in HEK293 cells co-transfected $\alpha 1 \mathrm{C}$ with HCN2-GFP. 


\section{Discussion}

FRAP is an optical technique that can be used to quantify two dimensionally molecular diffusion. The photobleaching of ROI allows adjacent molecules diffuse toward ROI in which the dynamic protein-protein interaction can be quantified by this approach. In the previous study, we have demonstrated that association between N-terminus of HCN2 and C-terminus of LTCC (Lin et al., 2010). In order to further confirm this association in a dynamic manner, we employed FRAP here to test the half-time of fluorescence recovery in HEK293 cells transfected with different recombinant proteins. If one protein is associated with another protein as a complex, the diffusion rate of the protein complex is slower than the protein by itself due to increase of the molecular weight. Our results revealed that the half-time in HEK293 cells co-expressed with the mutant (either Nterminal mutant of HCN2 or C-terminal mutant of LTCC) is significantly faster than that of the wild type, indicating N-terminus of HCN2 and C-terminus of LTCC are key contributors for the association between LTCC and HCN2.

It is worth to notice here that although the half-time of cells cotransfected $\alpha 1 \mathrm{C}$ with HCN2-GFP indicated in Fig 5 revealed faster a little bit when compared with $\alpha 1 \mathrm{C}$-GFP and $\mathrm{HCN} 2$ (Fig 2) $\left(\mathrm{t}_{1 / 2}=10 \pm 1.23(\mathrm{~s})<\mathrm{t}_{1 / 2}=14.04 \pm 3.51(\mathrm{~s}), \mathrm{n}=3\right)$, the value is significantly

slower than those of the mutants. This may be due to the larger molecular weight of $\alpha 1 \mathrm{C}$ ( 150KD) than HCN2 ( 100KD) (Huang et al., 2008; Lin et al., 2010). Our results strongly demonstrated that $\mathrm{HCN} 2$ associate with $\alpha 1 \mathrm{C}$ via the $\mathrm{N}$-terminus of $\mathrm{HCN} 2$ and IQ motif of $\alpha 1 \mathrm{C}$, indicating the crosstalk between these two channels on the cell membranes. 


\section{Overall Summary}

Up till now, several approaches have been developed to establish animal models for the study of arrhythmias. These include: (1) Aorta bandage to induce ischemic condition (Billman et al., 2005), (2) Installation of pacemakers to induce heart failure (Timek et al., 2003), and (3) Genetic ablation (Benkusky et al., 2007). Although widely used in many different studies, these model systems are all associated with structural remodeling, which results in ectopic automaticity or reentrant excitation and therefore induces cardiac arrhythmias.

However, structural remodeling is not the only mechanism for arrhythmias. It is known that SCD can also occur acutely (in several seconds) without structural remodeling (MacAlpin, 1993; Zipes et al., 1998), indicating that short-term dysregulation can also be an important arrhythmogenic mechanism. Therefore, it is of great value to establish an animal arrhythmic model for in the absence of structural remodeling. In Chapter 1, we successfully established a new rat arrhythmia model without structural remodeling, enabling research on mechanisms leading to short-term cardiac dysfunction.

\section{Alteration of cardiac rhythm by tyrosine phosphorylation}

In this study, we found that increasing tyrosine phosphorylation by applying tyrosine phosphatase inhibitor, sodium orthovanadate, led to different types of ventricular arrhythmias. Furthermore, reducing tyrosine phosphorylation by Src kinase inhibitor, PP2, significantly decreased heart rate as well as HRV. These results let us conclude that the endogenous tyrosine phosphorylation and dephosphorylation levels in the cardiac 
system delicately modulate the rhythm of the heart via balancing with each other in the physiological condition: Src-mediated tyrosine phosphorylation is dominant in atria whereas phosphatase-mediated tyrosine dephosphorylation is dominant in ventricles. That may explain why PP2 can reduce heart rate at a low dose $(0.2 \mathrm{mg} / \mathrm{kg})$ and induce AV block at a higher dose administration $(0.3 \mathrm{mg} / \mathrm{kg})$; whereas sodium orthovanadate can induce different types of ventricular arrhythmias at different doses $(3.8 \mathrm{mg} / \mathrm{kg}$ and $5.5 \mathrm{mg} / \mathrm{kg})$.

Although our main purpose of using PP2 is to decrease tyrosine phosphorylation (we have PP3 as the negative control), we cannot exclude the possibility that PP2 may directly affect other signal pathways, such as the pathways mediated by phosphatidyl inositol 3'-kinase (PI3K) and p38 mitogen-activated protein kinase (MAPK), the downstream of epidermal growth factor (EGF) receptor-mediated pathways (Kumar et al., 2003). Whether these kinases may also involve in the regulation of cardiac rhythm need further investigated.

The observed changes of cardiac rhythm and HRV by reducing tyrosine phosphorylation after administration of PP2 could be a result of 1) altered activity of autonomic nervous system (ANS), and/or 2) altered sensitivity of sinus node responding to ANS. Previous studies have demonstrated that $\operatorname{Src}$ family tyrosine kinases are associated with $\beta$ adrenergic receptors and regulate the internalization of these receptors (Huang et al., 2004). It is possible that $\mathrm{Src}$ kinases mediate $\mathrm{G}$ protein activation and alter the intracellular cAMP level leading to downstream signaling events. Therefore, inhibition of Src kinases by PP2 may interfere with and reduce the activity of ANS pathway. However, 
reducing ANS activity may not be the only explanation. The other possibility could be due to a reduced sensitivity of SA node to the ANS involving the alteration of ion channel activities. Previous studies showed that EGF increases heart rate and channel currents through tyrosine phosphorylation in the isolated heart system (Nair et al., 1993). Since HCN4 channels express dominantly in the sinus node contributing to the "U" turn in the phase 4 of sinus node action potential, one possible scenario is that $\mathrm{HCN}$ channel activities can be altered by tyrosine phosphorylation, thus mediating the change observed in the heart rate and HRV.

\section{Modulation of HCN channel activities by tyrosine phosphorylation}

To elucidate the possibility that the change of cardiac rhythm by tyrosine phosphorylation is due to the alteration of HCN channel activities, in chapter 2, we employed HEK293 cells co-expressing HCN channel proteins and other regulators. The advantage of using HEK293 cells is because this is a well-established expressing system without endogenous channel proteins, providing a clean platform to study channel proteins of our interest. Using this in vitro system combined with techniques of electrophysiology and confocal imaging, we were able to dissect the mechanism of phosphorylation-dependent regulation of heart rate and HRV.

Our results indicated that the increase of Src-mediated tyrosine phosphorylation dramatically enhanced surface expression and gating properties of $\mathrm{HCN}$ channels; conversely, RPTP $\alpha$, a tyrosine phosphatase, led to the decrease of surface expression and gating properties of channels by reducing tyrosine phosphorylation. In accordance with these results in HEK293 cells, our previous in vivo data using adult rat ventricular 
myocytes also revealed that increased tyrosine phosphorylation by sodium orthovanadate (a tyrosine phosphatase inhibitor) could enhance the current amplitude and gating properties of HCN channels (decrease the activation threshold from $-135 \mathrm{mv}$ to $-90 \mathrm{mv}$ ) in comparison with that in the absence of sodium orthovanadate (Huang et al., 2008). These data suggest that changed sensitivity of SA node to the ANS could indeed be a key contributor to the changed heart rate/ HRV when the phosphorylation level is altered.

Since HCN4 channels are dominantly expressed on atria whereas HCN2 channels on ventricles, altered activities of HCN channels increase the risk of ectopic automaticity by changing the slope in the phase 4 of atrial or ventricular action potential. If the automaticity of sinus node (primary pacemaker) is replaced by other regions of atriums or ventricles, atrial or ventricular arrhythmias could occur. This may explain why different types of ventricular arrhythmias can be induced as a response to the increased tyrosine phosphorylation by sodium orthovanadate.

On the other hand, reduced tyrosine phosphorylation state by PP2 may associate reduction of HCN4 channel activities and thus decrease the cardiac rhythm. However, there are still some questions that are not entirely understood, such as: 1) why Src kinases differentially modulate atrial activities whereas phosphatases dominantly regulate ventricular activities; 2) whether HCN4 channels can be downregulated on atrium by reduced tyrosine phosphorylation after administration of PP2; 3) whether HCN2 channels can be upregulated by increased tyrosine phosphorylation followed by application of sodium orthovanadate. These open questions acquire further investigations. 
Under pathological conditions, we also demonstrated that enhancement of tyrosine phosphorylation by three Src kinases, Src, Fyn, and Yes, can rescue the membrane trafficking defect mutant HCN4 D553N that has been found in a patient with long QT symptom followed by polymorphic ventricular tachycardia. By employing electrophysiology and confocal imaging, we found that not only membrane surface expression but gating properties and kinetics of this mutant can also be rescued after cotransfection of these three Src kinases. One possible explanation is that this single amino acid mutant (from $\mathrm{D}$ to $\mathrm{N}$ ) causes the loss of negative charges at the residue 553, which leads to conformation change (misfolding) and therefore interfering with trafficking and/or localizing to the surface membrane. These three Src kinases can provide phosphate, which is negatively charged, to tyrosine residues of this mutant and possibly prevent misfolding. Furthermore, our results also demonstrated that the subcellular location of $\mathrm{HCN} 4 \mathrm{D} 553 \mathrm{~N}$ mutant was at endoplasmic reticulum (ER), confirming that this mutant has membrane trafficking defect (Ueda et al., 2004).

Our biochemical results also showed that these three Src kinases have different tyrosine phosphorylation effects on the D553N mutant: Fyn has the highest whereas Yes has the lowest (Lin et al., 2009). It may explain why these three Src kinases have differential results: Yes and Src can rescue the surface expression whereas Fyn can accelerate the kinetics of this mutant. Nevertheless, the exact relationships among tyrosine phosphorylation, surface expression, and kinetics of the channel protein are not understood completely. Therefore, further studies are also needed in cardiac myocytes. 


\section{Calcium influx limitation of LTCC by HCN2}

We have also found a novel role of HCN2 in the regulation of LTCC inactivation. LTCCs contribute to the plateau phase of action potentials and delicately regulate the calcium influx. Since calcium homeostasis is pivotal for the maintenance of normal cardiac function, abnormal calcium handling can be directly linked to cardiac arrhythmias. Excessive calcium influx is known to lead to long QT interval and induce DAD-related tachyarrhythmias ((Burashnikov et al., 2003; Nattel et al., 1988; Satoh et al., 1998). EADs can also be induced if plateau potentials become abnormally prolonged (Chen et al., 2001; Coutu et al., 2006; Stambler et al., 2003).

In chapter 3, we found a new mechanism to restrict calcium influx via facilitation of inactivation of LTCCs. By electrophysiology after the cotransfection of $\alpha 1 \mathrm{C}$ (core subunit of LTCC) with HCN2 in HEK 293 cells, we concluded that HCN2 can induce faster inactivation of LTCCs when compared with the control. In order to investigate whether $\mathrm{CaM}$ is involved in this regulation, we used two structurally different $\mathrm{CaM}$ inhibitors, W-7 and TFP. Surprisingly, either W-7 or TFP can totally eliminate this fast inactivation of LTCCs triggered by HCN2. Similar results were also observed when we employed a CaM dominant negative mutant, $\mathrm{CaM}_{(1,2,3,4)}$. These data demonstrated that $\mathrm{CaM}$ is necessary in this HCN2-triggered fast inactivation, which is consistent with previous studies where CaM played the critical roles in the regulation of both VDI and CDI of LTCCs (Cens et al., 2006; Erickson et al., 2003; Kim et al., 2004; Pitt et al., 2001). The inhibitory time period of these two drugs is also comparable to that of previous studies (Reinhart et al., 1980; Hidaka et al., 1981). However, in the presence of 
$\alpha 2 \delta$ subunit, the role of CaM is compromised since neither $\mathrm{W}-7$ nor TFP affected this fast inactivation regulated by $\mathrm{HCN} 2$ any more, indicating the potential role of $\alpha 2 \delta$ in this regulation. One possibility is that the negative charges in the N-terminus of HCN2 may associate with the positive charges in the C-terminus of $\alpha 2 \delta$ and interfere with its interaction with $\mathrm{CaM}$. Besides, previous studies also revealed that $\alpha 2 \delta$ is pivotal to regulate the surface expression and gating properties of LTCCs (Arikkath et al., 2003; Felix et al., 1997; Yasuda et al., 2004), which is consistent with the reported finding here. In order to test the hypothesis that the fast inactivation of LTCCs initiated by HCN2 may depend on the interaction between N-terminus of HCN2 and IQ motif of $\alpha 1 \mathrm{C}$, we employed two mutants: N-terminal deleted HCN2 and IQ motif deleted $\alpha 1 \mathrm{C}$. Our results indicate that either of these two mutants could abolish or slow down this fast inactivation regulated by $\mathrm{HCN} 2$, suggesting that $\mathrm{N}$-terminus of $\mathrm{HCN} 2$ and IQ motif of $\alpha 1 \mathrm{C}$ are two key domains responsible for this regulation. Consistently, our results in FRAP further demonstrated the dynamic association between these two key domains, confirming the interaction between N-terminus of HCN2 and IQ motif of $\alpha 1 \mathrm{C}$.

\section{Conclusions}

In chapter 1 and chapter 2, the in vivo and in vitro results from my thesis work strongly indicated that tyrosine phosphorylation played a crucial role in the regulation of heart rate and cardiac arrhythmogenesis possibly through modulation of $\mathrm{HCN}$ channels. In chapter 3, we have demonstrated that the inactivation of LTCC, facilitated by HCN2 through the association of the two channels, leads to a reduced calcium influx. This provides 
scientific basis for potential therapeutic strategy for the treatment of cardiac arrhythmias (such as long QT syndrome) caused by excessive calcium homeostasis. 


\section{References}

Accili EA, Robinson RB, DiFrancesco D (1997) Am J Physiol Heart Circ Physiol 272: H1549-H1552.

Adams PR, Galvan M (1986) Voltage-dependent currents of vertebrate neurons and their role in membrane excitability. Adv Neurol 44: 137-170.

Altier C, Zamponi GW (2004) Trends Pharmacol Sci 25: 465-470.

Altomare C, Bucchi A, Camatini E, Baruscotti M, Viscomi C, Moroni A, DiFrancesco D (2001) J Gen Physiol 17: 519-532.

Anderson, CL, Balijepalli RC, Anson BD, Kamp TJ, January CT (2003) J Biol Chem 278: $35749-35754$.

Antal M, Papp I, Bahaerguli N, Veress G, and Vereb G (2004) Eur J Neurosci 19: 13361342.

Arikkath J, Campbell KP (2003) Curr Opin Neurobiol 13: 298-307.

Arinsburg SS, Cohen IS, Yu HG (2006) J Cardiovasc Pharmacol 47(4): 578-586.

Aubert AE, Seps B, Beckers F (2003) Sports Med 33(12): 889-919.

Barraclough J, Hodgkinson C, Hogg A, Dive C, Welman A (2007) Neoplasia 9: 745-754.

Bauer A, Kantelhardt JW, Barthel P, Schneider R, Ma“kikallio T, Ulm K, Hnatkova K, Scho“mig A, Huikuri H, Bunde A, Malik M, Schmidt G (2006) Lancet 367: 1674-1681.

Beggs AH, Sanguinetti MC, and Keating MT (2005) Proc Natl Acad Sci 102(23): 80898096

Benkusky NA, Weber CS, Scherman JA, Farrell EF, Hacker TA, Manorama CJ, Powers PA, and Valdivia HH (2007) Circulation Research 101: 819-829.

Bers BM (2000) Circ Res 87: 275-281.

Bers DM (2002) Nature 415: 198-205.

Billman GE (2002) Drug Development Research 55: 59-72.

Billman GE (2005) Practical Methods in Cardiovascular Research 1(1): 111-128.

Brown HF (1982) Physiol Rev 62: 505-530. 
Brumback AC, Lieber JL, Angleson JK, Betz WJ (2004) Methods 33(4): 287-294.

Brunetto AF, Roseguini BT, Silva BM, Hirai DM, Guedes DP (2005) Rev Bras Med Esporte 11(1): 22-27.

Burashnikov A, Antzelevitch C (2003) Circulation 107: 2355-2360.

Cens TM, Rousset JP, Fesquet LP, Charnet P (2006) Prog Biophys Mol Biol 90: 104-117.

Chaplan SR, Guo H-Q, Lee DH, Luo L, Liu C, Kuei C, Velumian AA, Butler MP, Brown SM, and Dubin AE (2003) J Neurosci 23: 1169-1178.

Cha TJ, Ehrlich JR, Chartier D, Xiao L, Nattel S (2006) Circulation 113: 1730-1737.

Chen J, Mitcheson JS, Lin M, Sanguinetti MC (2000) J Biol Chem 275: 36465-36471.

Chen YJ, Chen SA, Chen YC, Yeh HI, Chan P, Chang MS, and Lin CI (2001) Circulation 104: 2849-2854.

Cole GM, Bell L, Truong QB, Saitoh T (1992) Ann N Y Acad Sci 674: 103-117.

Coutu P, Chartier D, Nattel S (2006) Am J Physiol 291: H2290-H2300.

Dai DZ, Yu F (2005) Acta Pharmacol Sin 26(8): 918-925.

Daum G, Regenass S, Sap J, Schlessinger J, Fischer EH (1994) J Biol Chem 269: 1052410528.

Delisle BP, Anderson CL, Balijepalli RC, Anson BD, Kamp TJ, January CT (2003) $J$ Biol Chem 278: 35749-35754.

Delisle BP, Anson BD, Rajamani S, January CT (2004) Circ Res 94: 1418-1428.

Dewaard M, Strube C, Villaz M (1998) MS Med Sci 14: 764-770.

DiFrancesco D (1999) J Physiol 515: 367-376.

DiFrancesco D (1993) Annu Rev Physiol 55: 455-472.

Dobrev D, Milde AS, Andreas K, Ravens U (1999) Br J Pharmacol 127: 576-582.

Donnarumma L, Labbadia G, Paoletti V (2000) Adv Ther 17(5): 222-229.

Doval HC, Nul DR, Grancelli H, Varini SD, Soifer S, Sergio V (1996) Circulation 94: 3198-3203. 
Eric AJ, Reits, Neefjes JJ (2001) Nature Cell Biology 3: E145-147.

Erickson MG, Liang H, Mori MX, Yue DT (2003) Neuron 39: 97-107.

Felix R, Gurnett CA, De Waard M, Campbell KP (1997) J Neurosci 17: 6884-6891.

Fleckenstein A, Janke J, Doring HJ, Leder O (1974) Recent Adv Stud Card Struct Metab 4: 563-568.

Fogle KJ, Lyashchenko AK, Turbendian HK, Tibbs GR (2007) J Neurosci 27: 28022814.

Forder J, Scriabine A, Rasmussen H (1985) American Society for Pharmacology and Experimental Therapeutics 235: 267-273.

Furutani M, Trudeau MC, Hagiwara N, Seki A, Gong Q, Zhou Z, Imamura S, Nagashima H, Kasanuki H, Takao A, Momma K, January CT, Robertson GA, Matsuoka R (1999) Circulation 99: 2290-2294.

Gao T, Bunemann M, Gerhardstein BL, Ma H, Hosey MM (2000) J Biol Chem 275: 25436-25444.

Goldberger AL, Amaral LA, Hausdorff JM, IvanovPCh, Peng CK, Stanley HE (2002) Proc Natl Acad Si 99: 2466-2472.

Gordon JA (1991) Methods in Enzymology 201: 477-482.

Gustina AS, Trudeau MC (2011) J Gen Physiol 137 (3): 315-325.

Hanlon MR, Wallace BA (2002) Biochemistry 41: 2886-2894.

Hedley PL, Jorgensen P, Schlamowitz S (2009) Human Mutation 30(11): 1486-1511.

Hendrich J, Van Minh AT, Heblich F, Nieto-Rostro M, Watschinger K, Striessnig J, Wratten J, Davies A, Dolphin AC (2008) Proc Natl Acad Sci 105: 3628-3633.

Hidaka H, Sasaki Y, Tanaka T, Endo T, Ohno S, Fujii Y, Nagata T (1981) Proc Natl Acad Sci 78: 4354-4357.

Hodgkin AL, Huxley AF (1952) Journal of Physiology 116(4): 449-472.

Hodgkin AL, Huxley AF, Katz B (1952) Journal of Physiology 116(4): 424-448.

Hoffman BF, Rosen MR (1981) Circ Res 49: 1-15.

Huang J, Sun Y, and Huang XY (2004) J Biol Chem 279(20): 21637-21642. 
Huang JY, Huang A, Zhang Q, Lin YC, and Yu HG (2008) J Biol Chem 283: 2991229919.

Hunter JV, Moss AJ (2009) Neurology 72(3): 208-209.

Hynes BJ, Luck JC, Wolbrette DL, Boehmer J, Naccarelli GV (2002) Curr Treat Options Cardiovasc Med 4(6): 467-485

Ibba, M, Söll D (1999) Science 286: 1893-1897.

Irisawa H (1978) Physiol Rev 58: 461-498.

Ishii TM, Nakashima N, Takatsuka K, Ohmori H (2007) Biochemical and Biophysical Research Communications 359: 592-598.

Jouven X, Empana JP, Schwartz P, Desnos M, Courbon D, Ducimetie`re (2005) NEJM 352: 1951-1958.

Kalia LV, Gingrich JR, Salter MW (2004) Oncogene 23: 8007-8016.

Kanda S, Mochizuki Y, Nakamura T, Miyata Y, Matsuyama T, Kanetake H (2005) J Cell Sci 118: 961-970.

Kannel W, Cupples A, D’Agostino R (1987) Am Heart J 113: 799-804.

Kaplan R, Morse B, Huebner K, Croce C, Howk R, Ravera M, Ricca G, Jaye M, Schlessinger J (1990) Proc Natl Acad Sci 87: 7000-7004.

Kashem A, Hassan S, Crabbe DL, Melvin DB, Santamore WP (2003) J Thorac Cardiovasc Surg 125: 391-399.

Kim J, Ghosh S, Nunziato D, Pitt G (2004) Neuron 41: 745-754.

Kim JS, Ghosh DA, GS Pitt (2004) Neuron 41: 745-754.

Kitagawa J, Takeda M, Suzuki I, Kadoi J, Tsuboi Y, Honda K, Matsumoto S, Nakagawa H, Tanabe A, Iwata K (2006) Eur J Neurosci 24: 1976-1986.

Koh YH, Arnim CA, Hyman BT, Tanzi RE, Tesco G (2005) J Biol Chem 280(37): 32499-32504.

Kumar P, Amin MA, Harlow LA, Polverini PJ, and Koch AE (2003) Blood 101: 39603968.

Laitio TT, Huikuri HV, Kentala ES, Ma“kikallio TH, Jalonen JR, Helenius H, SariolaHeinonen K, Yli-Ma“yry S, Scheinin H (2000) Anesthesiology 93: 69-80. 
Laitio TT, Huikuri HV, Ma"kikallio TH, Jalonen JR, Kentala E, Helenius H, Pullisaar O, Hartiala J, Scheinin H (2004) Anesth Analg 98: 1239-1244.

Laitio TT, Makikallio TH, Huikuri HV, Kentala ES, Uotila P, Jalonen JR, Helenius H, Hartiala J, Yli-Mayry S, Scheinin H (2002) Am J Cardiol 89: 1176-1181.

Liang H, DeMaria CD, Erickson MG, Mori MX, Alseikhan BA, Yue DT (2003) Neuron 39: 951-960.

Li CH, Zhang Q, Teng BY, S. Mustafa J, Huang JY, Yu HG (2008) Am J Physiol Cell Physiol 294: C355-362.

Lin YC, Huang J, Kan H, Frisbee JC, Yu HG (2009) J Biol Chem 284(44): 30433-40.

Lin YC, Huang J, Zhang Q, Hollander JM, Frisbee JC, Martin KH, Nestor C, Goodman R, Yu HG (2010) Am J Physiol Cell Physiol. 298(5): C1029-1037.

MacAlpin (1993) Aamerican Heart Journal 125(4): 1011-1017.

Ma“kikallio TH, Barthel P, Schneider R, Bauer A, Tapanainen JM, Tulppo MP, Schmidt G, Huikuri HV (2005) Eur Heart J 26: 762-769.

Mammarella A, Paradiso M, Basili S, De Matteis A, Cardarello CM, Di Franco M, Donnarumma L, Labbadia G, Paoletti V (2000) Adv Ther 17(5): 222-229.

Mammarella A, Paradiso M, Basili S, De Matteis A, Cardarello CM, Di Franco M, Shinbane JS, Wood MA, Jensen DN (1997) J Am Coll Cardiol 29: 709-715.

Mammarella A, Paradiso M, Basili S, Matteis A, Cardarello CM, DiFranco M, Yasuda T, Chen L, Barr W, McRory JE, Lewis RJ, Adams DJ, Zamponi GW (2004) Eur J Neurosci 20: $1-13$.

McCleskey EW (1994) Curr Opin Neurobiol 4: 304-312.

Milanesi R, Baruscotti M, Gnecchi-Ruscone T, DiFrancesco D (2006) N Engl J Med 354: 151-157.

Moe GW, Stopps TP, Howard RJ, Armstrong PW (1998) J Lab Clin Med 112: 426-432.

Mohler PJ, Wehrens X (2007) Physiology 22(5): 342-350.

Morita H, Wu J, Zipes DP (2008) Lancet 372 (9640): 750-763.

Moss AJ, Schwartz PJ (2005) Circulation 111(9): 1199-1201. 
Myerburgh RJ, Interian A Jr, Mitrani RM, Kessler KM, Castellanos A (1997) Am J Cardiol 80: 10F-19F.

Nair BG, Rashed HM, and Patel TB (1993) Growth Factors 8(1): 41-48.

Nattel S, Maguy A, Le Bouter S, Yeh YH (2007) Physiol Rev 87: 425-456.

Nattel S, Quantz MA (1988) Cardiovasc Res 22: 808-817.

Noble D (1960) Nature 188: 495-497.

Nof E, Luria D, Brass D, Marek D, Lahat H, Reznik-Wolf H, Pras E, Dascal N, Eldar M, Glikson M (2007) Circulation 116: 463-470.

Novotny MJ, and Adams HR (1986) J Am Vet Med Assoc 189(5): 533-539.

Osawa M, Swindells MB, Tanikawa J, Tanaka T, Mase T, Furuya T, Ikura M (1998) J Mol Biol 276: 165-176.

Papp I, Szucs P, Hollo K, Erdelyi F, Szabo G, Antal M (2006) Eur J Neurosci 24: 13411352.

Paulussen A, Raes A, Matthijs G, Snyders DJ, Cohen N, Aerssens J (2002) J Biol Chem 277: 48610-48616.

Peng CK, Havlin S, Stanley HE, Goldberger AL (1995) Chaos 1: 82-87.

Peng CK, Mietus J, Hausdorff JM, Havlin S, Stanley HE, Goldberger AL (1993) Phys Rev Lett 70: 1343-1346.

Peterson BZ, DeMaria CD, Adelman JP, Yue DT (1999) Neuron 22: 549-558.

Pian P, Bucchi A, Robinson RB, Siegelbaum SA (2006) J Gen Physiol 128: 593-604.

Pitt GS, Zuhlke RD, Hudmon A, Schulman H, Reuter H, Tsien RW (2001) J Biol Chem 276: 30794-30802.

Ponniah S, Wang DZ, Lim KL, Pallen CJ (1999) Curr Biol 9: 535-538.

Poolos NP, Bullis JB, Roth MK (2006) J Neurosci 26: 7995-8003.

Proenza C, Tran N, Angoli D, Zahynacz K, Balcar P, Accili EA (2002) J Biol Chem 277: 29634-29642.

Pumprla J, Howorka K, Groves D, Chester M, Nolan J (2002) Int J Cardiol 84(1): 1-14. 
RajendraAcharya U, Paul JK, Kannathal N, Lim CM, Suri JS (2006) Med BioEng Comput 44(12): 1031-1051.

Reinhart PH, Taylor WM, Bygrave FL (1980) FEBS Lett 120: 71-74.

Robert S (2005) Journal of Clinical Investigation 115 (8): 1986-1989.

Robinson RB, Siegelbaum SA (2003) Annu Rev Physiol 65: 453-480.

Santoro B, Baram TZ (2003) Trends Neurosci 26: 550-554.

Santoro B, Chen S, Luthi A, Pavlidis P, Shumyatsky GP, Tibbs GR, Siegelbaum SA (2000) J Neurosci 20: 5264-5275.

Santoro B, Grant SG, Bartsch D, Kandel, ER (1997) Proc Natl Acad Sci 94: 1481514820 .

Satoh T, Zipes DP (1998) J Cardiovasc Electrophysiol 9: 970-975.

Schulze-Bahr E, Neu A, Friederich P, Kaupp UB, Breithardt G, Pongs O, Isbrandt D (2003) J Clin Invest 111: 1537-1545.

Schwartz PJ (1998) Eur Heart J 19: F72-80.

Schwartz PJ, Joseph RM, Condouris K, Tager-Flusberg H, Priori SG, Sanguinetti MC, Keating MT (2004) Cell 119(1): 19-31.

Schwartz PJ, La Rovere MT, Vanoli E (1992) Circulation 85: I77-91.

Scmiddt G, Malik M, Barthei P, Schneider R, Ulm K, Rolnitzky L, Camm AJ, Bigger JT, Scho"mig A (1999) Lancet 353: 1390-1396.

Shi W, Wymore R, Yu H, Wu J, Wymore RT, Pan Z, Robinson RB, Dixon JE, McKinnon D, Cohen IS (1999) Circ Res 85: E1-6.

Smith AL, Reynolds KJ, Owen H (2007) Australas Phys Eng Sci Med. 30(4): 336-341.

Splawski I, Timothy KW, Sharpe LM, Decher N, Kumar P, Bloise R, Napolitano C, Tsien RW, Lipscombe D, Madison D, Bley K and Fox A (1995) Trends Neurosci 18: 5254.

Sprague BL, McNally JG (2005) Trends in Cell Biology 15(2): 84-91

Staes M, Talavera K, Klugbauer N, Prenen J, Lacinova L, Droogmans G, Hofmann F, Nilius B (2001) Journal of Physiology 530(1): 35-45. 
Stambler BS, Fenelon G, Shepard RK, Clemo HF, Guiraudon CM (2003) J Cardiovasc Electrophysiol 14: 499-507.

Stein PK, Schmieg RE, El-Fouly A, Domitrovich PP, Buchman TG (2001) Crit Care Med 29: 1738-1743.

Stieber J, Herrmann S, Feil S (2003) Proc Natl Acad Sci 100: 15235-15240.

Stoker AW (2005) J Endocrinol 185: 19-33.

Su J, Muranjan M, Sap J (1999) Curr Biol 9: 505-511.

Thuraisingham RA (2006) Comput Methods Programs Biomed 83(1): 78-82.

Thomas SM, Brugge JS (1997) Annu Rev Cell Dev Biol 13: 513-609.

Timek TA, Dagum P, Lai DT (2003) J Thorac Cardiovasc Surg 125: 315-324.

Tsien RW, Lipscombe D, Madison D, Bley K, Fox A (1995) Trends Neurosci 18: 52-54.

Ueda K, Nakamura K, Hayashi T, Inagaki N, Takahashi M, Arimura T, Morita H, Higashiuesato Y, Hirano Y, Yasunami M, Takishita S, Yamashina A, Ohe T, Sunamori M, Hiraoka M, Kimura A (2004) J Biol Chem 279: 27194-27198.

VladIanus Y, Zhao TC, Tseng A, Padbury JF, Tseng YT (2007) Am J Physiol Heart CircPhysiol 293: H385-H393.

Wang HG, George MS, Kim J, Wang C, Pitt GS (2007) J Neurosci 27: 9086-9093.

Wang Q, Brunner HR, Burnier M (2004) Am J Physiol Heart Circ Physiol 286: H806814.

Weibel ER (1991) Am J Physiol 261: L361-369.

Westenbroek RE, Ahlijanian MK, Catterall WA (1990) Nature 347: 281-284.

Wickner S, Maurizi MR, Gottesman S (1999) Science 286: 1888-1893.

Williams EM (1984) J Clin Pharmacol 24(4): 129-147.

Willich S, Levy D, Rocco M, Tofler G, Stone P, Muller J (1987) Am J Cardiol 60: 801806.

Wolfe CL, Nibley C, Bhandari A, Chatteriee K, Scheinman M (1991) Circulation 84(4): 1543-1551. 
Wrogemann K, Pena SD (1976) Lance 1: 672-674.

Wu JY, Yu HG, Cohen IS (2000) Biochimica Biophysica Acta 463: 15-19.

Wu ZK, Vikman S, Laurikka J, Pehkonen E, Iivainen T, Huikuri H, Tarkka M (2005) Eur J Cardio Thoracic Surg 28: 109-13.

Xia XM, Fakler B, Rivard A, Wayman G, Johnson-Pais T, Keen JE, Ishii T, Hirschberg B, Bond CT, Lutsenko S, Maylie J, Adelman JP (1998) Nature 395: 503-507.

Yao H, Donnelly DF, Ma C, LaMotte RH (2003) J Neurosci 23: 2069-2074.

Yasuda T, Chen L, Barr W, McRory JE, Lewis RJ, Adams DJ, Zamponi GW (2004) Eur J Neurosci 20: 1-13.

Yue L, Feng J, Gaspo R, Li GR, Wang Z, Nattel S (1997) Circ Res 81: 512-525.

Yu HG, Lu ZJ, Pan ZM, Cohen IS (2004) Pflugers Arch 447: 392-400.

Yu HG, Wu J, Potapova I, Wymore RT, Holmes B, Zuckerman J, Pan Z, Wang H, Shi W, Robinson RB, El-Maghrabi MR, Benjamin W, Dixon J, McKinnon D, Cohen IS, Wymore R (2001) Circ Res 88: E84-87.

Yu X, Chen XW, Zhou P, Yao L, Liu T, Zhang B, Li Y, Zheng H, Zheng LH, Zhang CX, Bruce I, Ge JB, Wang SQ, Hu ZA, Yu HGc-CA, Zhou Z (2007) Am J Physiol Cell Physiol 292: C1147-1155.

Yu X, Duan KL, Shang CF, Yu HG, Zhou Z (2004) Proc Natl Acad Sci 101: 1051-1056.

Zagotta WN, Olivier NB, Black KD, Young EC, Olson R, Gouaux E (2003) Nature 425: 200-205.

Zhang R, Dzhura I, Grueter CE, Thiel W, Colbran RJ, Anderson ME (2005) FASEB J 19: 1573-1575.

Zhou J, Spier SJ, Beech J, Hoffman EP (1994) Hum Mol Genet. 3(9): 1599-1603.

Zhou Z, Gong Q, EpsteinML, January CT (1998) J Biol Chem 273: 21061-21066.

Zhou Z, Gong Q, and January CT (1999) J Biol Chem 274: 31123-31126.

Zipes DP, Wellens HJ (1998) Circulation 98: 2334-2351.

Zong X, Eckert C, Yuan H, Wahl-Schott C, Abicht H, Fang L, Li R, Mistrik P, Gerstner A, Much B, Baumann L, Michalakis S, Zeng R, Chen Z, Biel M (2005) J Biol Chem 280: 34224-34232. 
Zuhlke RD, Pitt GS, Deisseroth K, Tsien RW, Reuter H (1999) Nature 399: 159-162.

Zuhlke RD, Reuter H (1998) Proc Natl Acad Sci 95: 3287-3294. 


\section{Curriculum Vitae}

\section{A. Personal Details}

Name: Yen-Chang Lin

Address: Department of Physiology and Pharmacology Center for Cardiovascular and Respiratory Sciences, West Virginia University School of Medicine, Morgantown, WV 26506-9229

Phone: (304) 293-1019

Email: ylin@hsc.wvu.edu

Birth date: June 7, 1976

Place of Birth: Taiwan

\section{B. Education}

1995 - 1999 B.S. Biology, Tung Hai University, Taiwan.

1999 - 2001 M.S. Neuroscience, National Tsing Hua University, Taiwan.

2006-present Predoctoral graduate student, Physiology and Pharmacology, West Virginia University, USA.

\section{Professional Experience}

1997 1999 Student Research Assistant, Tung Hai University, Taiwan.

1999-2001; 2003-2004 Student Research Assistant, Department of Neuroscince, National Tsing Hua University, Taiwan.

2006-2011 Graduate Research Assistant, West Virginia University, USA.

2010-2011 Student Research Assistant, National Institute for Occupational Safety and Health (NIOSH), USA. 


\section{Selected peer-reviewed publications (in chronological order)}

1. Yan-Chiang Lin, Zu-Han Huang, I-Sam Jan, Chia-Chun Yeh, Han-Jay Wu, Yun-Chia Chou, and Yen-Chung Chang. Development of excitatory synapses in cultured neurons dissociated from the cortices of rat embryos and rat pups at birth. J. Neurosci. Res. 67: 484-93, 2002.

2. Jianying Huang, Aijie Huang, Qi Zhang, Yen-Chang Lin, Han-Gang Yu. Novel Mechanism for Suppression of HCN Pacemaker Channels by Receptor-like Tyrosine Phosphatase-alpha. J. Biol. Chem. 283: 29912-29919, 2008.

3. Qi Zhang, Aijie Huang, Yen-Chang Lin, Han-Gang Yu. Associated Changes in HCN2 and HCN4 Transcripts and I(f) Pacemaker Channels in Myocytes. BBA-Biomembrane, 1788: 1138-1147, 2009.

4. Han-Gang Yu, Jianying Huang, Yen-Chang Lin. Non-proteolytic HCN2 in the heart. J. Biol. Chem. 284, le7, 2009.

5. Yen-Chang Lin, Jianying Huang, Kan Hong, Jefferson Frisbee, Han-Gang Yu. Rescue of a Trafficking Defective Human Pacemaker Channel via a Novel Mechanism: Roles of Src, Fyn, Yes tyrosine kinases. J. Biol. Chem. 284: 30433-30440, 2009.

6. Yen-Chang Lin, Jianying Huang, Qi Zhang, John M. Hollander, Jefferson C. Frisbee, Karen H. Martin, Casey Nestor, Robert Goodman, Han-Gang Yu. Modulation of Ltype Calcium Channel by HCN2 Channel. Am J Physiol Cell Physiol. 298(5): C102937, 2010. 


\section{E. International Meeting and Poster}

1. Hang-Gang Yu, Chenhong Li, Yen-Chang Lin, and Qi Zhang. "Differential Modulation by Src Tyrosine kinase of HCN channels". In Proceedings of the Experimental Biology convention, San Diego, CA, April 6-9, 2008.

2. Jianying Huang, Aijie Huang, Qi Zhang, Yen-Chang Lin, Han-Gang Yu. "Novel Mechanism for Suppression of HCN Pacemaker Channels by Receptor-like Tyrosine Phosphatase-alpha”. Biophysical Society $53^{\text {th }}$ Annual Meeting, Boston, MA, February 28-March 4, 2009.

3. Yen-Chang Lin, Jianying Huang, Hong Kan, Jefferson Frisbee, Han-Gang Yu. "Rescue of a Trafficking Defective Human Pacemaker Channel via a Novel Mechanism: Roles of Src, Fyn, Yes tyrosine kinases”. Biophysical Society $54^{\text {th }}$ Annual Meeting, San Francisco, CA, February 20-24, 2010 (Poster Presentation).

4. Yen-Chang Lin, Jianying Huang, Hong Kan, Adam Goodwill, Jefferson Frisbee, Han-Gang Yu. "Disrupted Inactivation of Cardiac L-type Calcium Channel in Obese Zucker Rat”. American Heart Association scientific session meeting, 2011 (abstract).

\section{F. Presentations}

1. "The regulation of L-type calcium channel inactivation", seminar presentation, Integrative physiology and pharmacology department, WVU, April, 2009.

2. "Teaching Practicum" course-Teaching Demo, Integrative Physiology and Pharmacology Department, WVU, Spring, 2009.

3. "Novel Mechanisms for Ion Channel Modulation", seminar presentation, Integrative physiology and pharmacology department, WVU, March, 2010. 
4. "Rescue of a Trafficking Defective Human Pacemaker Channel via a Novel Mechanism: Roles of Src, Fyn, Yes tyrosine kinases", Annual Van Liere Convocation poster presentation, WVU, April, 2010.

5. "Rescue of a Trafficking Defective Human Pacemaker Channel via a Novel Mechanism: Roles of Src, Fyn, Yes tyrosine kinases", Annual Julie Bestchard Symposium presentation, Integrative physiology and pharmacology department, WVU, May, 2010.

6. "A Novel Role of Tyrosine Phosphorylation in Cardiac Arrhythmogenesis in vivo", seminar presentation, Integrative physiology and pharmacology department, WVU, April, 2011.

7. "Establishment of a Rat Model for Studying Ventricular Arrhythmias", Annual Van Liere Convocation poster presentation, WVU, February, 2011. 


\section{Appendix}

My published papers are attached in the appendix, which are entitled as:

1) Rescue of a trafficking defective human pacemaker channel via a novel mechanism:

role of Src, Fyn, and Yes tyrosine kinases.

2) Inactivation of L-type calcium channel modulated by HCN2 channel. 


\section{Rescue of a Trafficking Defective Human Pacemaker Channel via a Novel Mechanism ROLES OF SrC, Fyn, AND Yes TYROSINE KINASES*[s]}

Received for publication, June 29, 2009, and in revised form, September 10, 2009 Published, JBC Papers in Press, September 11, 2009, DOI 10.1074/jbc.M109.039180 Yen-Chang Lin ${ }^{\ddagger \S 1}$, Jianying Huang ${ }^{\ddagger \S 1}$, Hong Kan ${ }^{\ddagger \uparrow}$, Jefferson C. Frisbee ${ }^{\ddagger \S}$, and Han-Gang $\mathrm{Yu}^{\ddagger \S 2}$ From the ${ }^{\ddagger}$ Center for Cardiovascular and Respiratory Sciences and the Departments of ${ }^{\S}$ Physiology and Pharmacology and "Cardiology, West Virginia University School of Medicine, Morgantown, West Virginia 26506

Therapeutic strategies such as using channel blockers and reducing culture temperature have been used to rescue some long QT-associated voltage-gated potassium Kv trafficking defective mutant channels. A hyperpolarization-activated cyclic nucleotidegated HCN4 pacemaker channel mutant (D553N) has been recently found in a patient associated with cardiac arrhythmias including long QT. D553N showed the defective trafficking to the cell surface, leading to little ionic current expression (loss-of-function). We show in this report that enhanced tyrosine phosphorylation mediated by Src, Fyn, and Yes kinases was able to restore the surface expression of D553N for normal current expression. Src or Yes, but not Fyn, significantly increased the current density and surface expression of D553N. Fyn accelerated the activation kinetics of the rescued D553N. Co-expression of D553N with Yes exhibited the slowest activation kinetics of D553N. Src, Fyn, and Yes significantly enhanced the tyrosine phosphorylation of D553N. A combination of Src, Fyn, and Yes rescued the current expression and the gating of $\mathrm{D} 553 \mathrm{~N}$ comparable with those of wild-type HCN4. In conclusion, we demonstrate a novel mechanism using three endogenous Src kinases to rescue a trafficking defective HCN4 mutant channel (D553N) by enhancing the tyrosine phosphorylation of the mutant channel protein.

Defective trafficking leading to the reduced surface expression of ion channels is one of the mechanisms responsible for a loss-of-function of the ion channel on the plasma membrane (1). Several methods have been developed to rescue the voltagegated potassium Kv trafficking defective channels: reducing the culture temperature, applying the channel blockers, altering the molar ratio of glycerol, and using the sarcoplasmic/endoplasmic reticulum $\mathrm{Ca}^{2+}$-ATPase inhibitor thapsigargin (2-6).

Hyperpolarizing-activated cyclic nucleotide-gated $(\mathrm{HCN})^{3}$ pacemaker channels generate time- and voltage-dependent

* This work was supported, in whole or in part, by National Institutes of Health Grant HL075023. This work was also supported by the Office of Research and Graduate Programs/Health Sciences Center at West Virginia University (to H.-G. Y.).

5 The on-line version of this article (available at http://www.jbc.org) contains supplemental Figs. S1 and S2.

${ }^{1}$ Both authors contributed equally to this work.

${ }^{2}$ To whom correspondence should be addressed: One Medical Center Dr., Rm. 3034/HSN, Dept. of Physiology, West Virginia University, Morgantown, WV 26506. Tel.: 304-293-2324; Fax: 304-293-5513; E-mail: hyu@ hsc.wvu.edu.

${ }^{3}$ The abbreviations used are: $\mathrm{HCN}$, hyperpolarizing-activated cyclic nucleotide-gated; RPTP, receptor-like protein tyrosine phosphatase; PBS, phosphate-buffered saline. inward currents, named $I_{\mathrm{h}}$ in neurons or $I_{\mathrm{f}}$ in the heart (7). They are important in various cell functions including excitability, synapse transmission, and rhythmic activity (7). The most well studied regulation of $I_{\mathrm{f}}$ is its response to autonomic stimulation. $\beta$-Adrenergic receptor activation increases and acetylcholine receptor activation decreases the intracellular cAMP levels, which in turn increases/decreases $I_{\mathrm{f}}$ by binding to the cyclic nucleotide-binding domain of the $\mathrm{HCN}$ channels, respectively (7). Other important mechanisms for the modulation of $I_{\mathrm{f}} / \mathrm{HCN}$ channels have recently been found including $\beta$-subunit $(8)$, lipids $(9,10)$, and p38 mitogen-activated protein kinase (11).

Accumulating evidence has revealed tyrosine phosphorylation as an important mechanism for modulation of HCN channel properties (12-16). An acute increase in tyrosine phosphorylation of $I_{\mathrm{f}}$ or $\mathrm{HCN}$ channels increases the channel activity, including an increase in the current amplitude, a positive shift of the voltage-dependent activation, an acceleration of activation kinetics, and an increase in whole cell conductance (1215). Recently, we discovered that the cell surface expression of HCN2 channels can be remarkably inhibited by tyrosine dephosphorylation mediated by receptor-like protein tyrosine phosphatase $\alpha$ (RPTP $\alpha)$ and increased by tyrosine phosphorylation via Src kinase after long term treatment (17).

D553N, a missense HCN4 mutant, was recently identified in a patient with cardiac arrhythmia associated with depressed HCN gating properties (18). Functional and structural assays revealed that D553N expresses little ionic currents, which is possibly due to the defective channel trafficking so that the channels cannot reach the plasma membrane for normal functions (18).

The Src kinase family has nine members (19). They are closely related and share the same regulatory function. Three of them, Src, Fyn, and Yes, are ubiquitously expressed in a variety of tissues including neurons and myocytes (19, 20). Without stimulation, they are inactive. However, mutation of key tyrosine residue results in the constitutively active form of the kinase, SrcY529F, FynY531F, and YesY537F, respectively (15, 21, 22). Using these Src kinases, we show in this report a novel approach that can restore the surface expression of D553N for normal current expression via tyrosine phosphorylation.

\section{EXPERIMENTAL PROCEDURES}

cDNA Plasmids-The human version of HCN4-pcDNA1.1 was kindly provided by Dr. U. B. Kaupp and subcloned into pcDNA3.1 vector. HCN4-D553N-pcDNA3 was made by sub- 
stituting aspartic acid with asparagine using PCR. HCN4D553N-DsRed was made by subcloning HCN4-D553N into the DsRed vector. RPTP $\alpha$ was a generous gift from Dr. Jan Sap (University of Copenhagen, Copenhagen, Denmark). The constitutively active form of Fyn, FynY531F, in pcDNA3.1 vector, was kindly provided by Dr. Shigeru Kanda (Nagasaki University, Nagasaki, Japan). The constitutively active form of Yes (YesY537F) was kindly provided by Dr. Arkadiusz Welman (Edinburgh Cancer Research Center), and we subcloned it into the pcDNA3.1 vector. Src529 (SrcY529F) was purchased from Upstate Biotechnology (Millipore). For simplicity, we also use Src, Fyn, and Yes and Src529, Fyn531, and Yes537 in the text for interchangeable use with the constitutively active form of each kinase: SrcY529F, FynY531F, and YesY537F.

Cell Culture and Plasmids Transfection-HEK293 cells were grown in Dulbecco's modified Eagle's medium (Invitrogen), supplemented with $10 \%$ fetal bovine serum, $100 \mathrm{IU} / \mathrm{ml}$ penicillin, and $100 \mathrm{~g} /$ liter streptomycin. Cells with 50-70\% confluence in 6-well plates were used for plasmid transfection using Lipofectamine2000 (Invitrogen).

Cell Lysis, Immunoprecipitation, and Western Blot AnalysisTotal protein extracts were prepared from cells transfected for $24-48$ h using CelLytic cell lysis reagent (Sigma) supplemented with protease inhibitors. For membrane fraction preparations, we used a membrane protein extraction kit (Pierce). The protein concentration of the lysate was determined using the Bradford or BCA assay. Equal amounts of total protein $(0.5-1 \mathrm{mg})$ were incubated with a specific antibody for $1 \mathrm{~h}$ at $4{ }^{\circ} \mathrm{C}$, and protein A/G Plus-agarose (Santa Cruz) was then added and incubated overnight with gentle rocking. The beads were washed three times with cold PBS buffer and resuspended in $2 \times$ sample buffer. The immune complexes were separated by SDS-PAGE and analyzed by Western blot using the specific antibody of interest. Total protein of 5-20 $\mu \mathrm{g} / \mathrm{sample}$ was subjected to SDS-PAGE using 4-12\% gradient gels (Invitrogen) and then transferred to nitrocellulose membranes (Amersham Biosciences) and incubated with proper antibodies. After washing and incubating with horseradish peroxidase-conjugated secondary antibody, immunoreactive proteins were visualized with the SuperSignal ${ }^{\circledR}$ West Pico kit (Pierce).

Cell Surface Biotinylation-Cell surface biotinylation experiments were performed by following the manufacturer's instruction (Pierce). Briefly, HEK293 cells transfected with desired plasmids were first treated with EZ-link Sulfo-NHS-SSBiotin to label cell surface proteins. The cells were subsequently lysed with lysis buffer containing protease inhibitor mixture (Sigma). The labeled proteins were then isolated with immobilized NeutrAvidin-agarose. After washing three times, the bound proteins were released by incubating with SDS-PAGE sample buffer containing $50 \mathrm{~mm}$ dithiothreitol and then analyzed by Western blotting. All of the protein experiments were repeated at least three times.

Whole Cell Patch Clamp Recordings-For recording $I_{\mathrm{HCN} 4}$, day $1(24-30 \mathrm{~h})$ up to day $4(90-98 \mathrm{~h})$ post-transfection HEK293 cells with green fluorescence were selected for patch clamp studies. The HEK 293 cells were placed in a Lucite bath in which the temperature was maintained at $25 \pm 1{ }^{\circ} \mathrm{C}$ by a temperature controller (Cell MicroControls). $I_{\mathrm{HCN} 4}$ currents were recorded using the whole cell patch clamp technique with an Axopatch-200B amplifier. The current amplitude of HCN4 or D553N current is defined as the amplitude of the onset timedependent inward current elicited by the hyperpolarizing pulse, excluding the instant jump at the beginning of the pulse. The current density is the current amplitude divided by the cell capacitance measured in each cell studied. The pipettes had a resistance of 2-4 M $\Omega$ when filled with internal solution: $6 \mathrm{~mm}$ $\mathrm{NaCl}, 130 \mathrm{~mm}$ potassium aspartate, $2 \mathrm{~mm} \mathrm{MgCl}_{2}, 5 \mathrm{~mm} \mathrm{CaCl}_{2}$, $11 \mathrm{~mm}$ EGTA, and $10 \mathrm{~mm}$ HEPES; pH was adjusted to 7.2 by $\mathrm{KOH}$. The external solution contained $120 \mathrm{~mm} \mathrm{NaCl}, 1 \mathrm{~mm}$ $\mathrm{MgCl}_{2}, 5 \mathrm{~mm}$ HEPES, $30 \mathrm{~mm} \mathrm{KCl}, 1.8 \mathrm{~mm} \mathrm{CaCl}_{2}$; $\mathrm{pH}$ was adjusted to 7.4 by $\mathrm{NaOH}$. The $I_{\text {to }}$ blocker, 4-aminopyridine (2 $\mathrm{mm})$, was added to the external solution to inhibit the endogenous transient potassium current, which can overlap with and obscure $I_{\mathrm{HCN} 4}$ tail currents recorded at $+20 \mathrm{mV}$. The data were acquired by CLAMPEX and analyzed by CLAMPFIT (pClamp 8; Axon).

The data are shown as the means \pm S.E. The threshold activation of $I_{\mathrm{HCN} 4}$ is defined as the first hyperpolarizing voltage at which the first time-dependent inward current can be observed. Student's $t$ test was used for statistical analysis with $p<0.05$ being considered statistically significant. Time constants were obtained by using Boltzmann best fit with one exponential function on current traces that reach steady state. HCN4 activates slowly, and the cells would not tolerate pulses sufficiently long to reach the steady state. We therefore used the following approach to obtain an accurate estimate of the steady state activation (15). The onset current traces were fitted with a single exponential function to $30-40 \mathrm{~s}$ to allow estimates of steady state current levels. The fitted current amplitudes were then divided by the driving force (the difference between test pulses and the reversal potential that was measured in each cell) to obtain the conductance at each test pulse. The activation curves were constructed by normalizing the conductance to its maximal value in response to the most negative test pulse.

Confocal Fluorescent Imaging of HEK293 Cells-HEK293 cells transfected with HCN4-DsRed or HCN4-DsRed-D553N were incubated on coverslips and fixed in $4 \%$ paraformaldehyde/PBS for $15 \mathrm{~min}$ and then washed with PBS (10 mM phosphate buffer, $150 \mathrm{~mm} \mathrm{NaCl}, \mathrm{pH} \mathrm{7.4)} \mathrm{for} 5 \mathrm{~min}$ for three times, followed by blocking in $1 \%$ bovine serum albumin/PBS, $\mathrm{pH} 7.4$, for $60 \mathrm{~min}$. After washing six times in PBS, the coverslips were mounted on slide glasses using Fluoromount G (Southern Biotechnology). The cells were imaged by a LSM510 confocal microscopy using a Plan-Neofluar $40 \times / 0.75$ objective or a Plan-Apochromat $63 \times / 1.4$ Oil differential interference contrast M27 objective. For DsRed imaging, a 1.2-milliwatt 543-nm HeNe laser was used for excitation, and a 560-615-nm BP emission filter was used for emission.

\section{RESULTS}

Inhibition of HCN4 Current Expression by RPTP $\alpha-$ We have recently demonstrated that $\operatorname{RPTP} \alpha$ can inhibit the surface expression of HCN2 channels via tyrosine dephosphorylation (17). Given the high structural homology between HCN2 and HCN4 (>80\%) (7), it was expected that RPTP $\alpha$ may also inhibit the surface expression of HCN4. Fig. 1 shows a typical current 


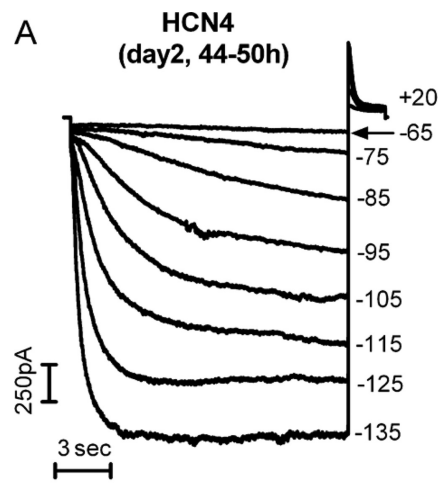

C

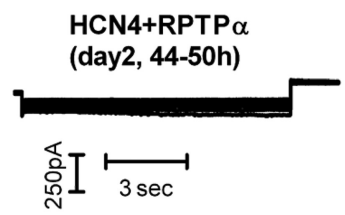

B

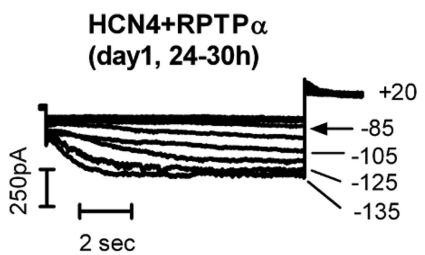

D $\quad$ HCN4+pRK5 (day2, 44-50h)

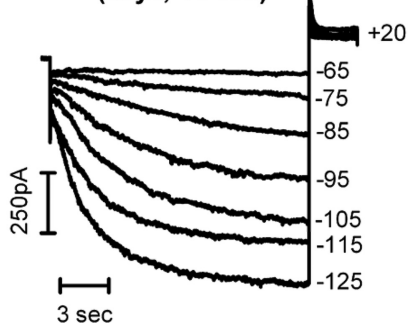

FIGURE 1. RPTP $\alpha$ on HCN4 current expression in HEK293 cells. A, HCN4 current expression in response to 15-s hyperpolarizing pulses from -65 to $-135 \mathrm{mV}$ in 10-mV increments. Tail currents were recorded at $+20 \mathrm{mV}$. $B$, in a cell co-transfected with RPTP $\alpha$ after 1 day of incubation, HCN4 current expression in response to 10-s hyperpolarizing pulses from -65 to $-135 \mathrm{mV}$ in 10-mV increments. $C$, in a cell co-transfected with RPTP $\alpha$ after 2 days of incubation, HCN4 current expression in response to 10-s hyperpolarizing pulses from -65 to $-135 \mathrm{mV}$ in $10-\mathrm{mV}$ increments. $D$, HCN4 current expression in HEK293 cells co-expressed with the empty vector, pRK5.

expression of HCN4 expressed in HEK293 cells (Fig. 1A). The expression was dramatically suppressed across the test voltages $(-65$ to $-135 \mathrm{mV}$ ) when HCN4 was co-expressed with RPTP $\alpha$ after 1 day of transfection (Fig. 1B). The reduction in current expression was associated with a negative shift in threshold activation (Figs. 1, $A$ and $B$, arrows). After 2 days of transfection, $\operatorname{RPTP} \alpha$ almost eliminated the current expression of HCN4 (Fig. 1C). The effect of RPTP $\alpha$ on HCN4 current expression is similar to that on HCN2 current expression (17). As a control, the empty vector, pRK5 (used to subclone RPTP $\alpha$ ), did not affect the current expression of HCN4 (Fig. 1D). Each of these results was confirmed in an additional 5-7 cells.

Inhibited Surface Expression and Reduced Tyrosine Phosphorylation of HCN4 by RPTP $\alpha$-Wondering whether $\mathrm{HCN} 4$ current inhibition of RPTP $\alpha$ is due to the suppressed membrane expression of the channel proteins, we examined the HCN4 channel membrane preparation (Fig. 2A). The left triplet shows $\mathrm{HCN} 4$ expression. The split bands indicate unglycosylated and glycosylated forms, similar to $\mathrm{HCN} 2$ membrane expression (17). The glycosylated form of $\mathrm{HCN} 4$ was significantly inhibited by RPTP $\alpha$ (middle triplet) and enhanced by Src529 (a constitutively active form of Src) (right triplet).

Using the phosphotyrosine-specific antibody 4G10, Fig. $2 B$ shows that the tyrosine phosphorylation of HCN4 channel protein (middle lane) was significantly enhanced by Src529 (second lane from the right) but inhibited by $\operatorname{RPTP} \alpha$ (left lane). These results suggested that the altered membrane expression of $\mathrm{HCN} 4$ is possibly caused by the increased or decreased tyrosine

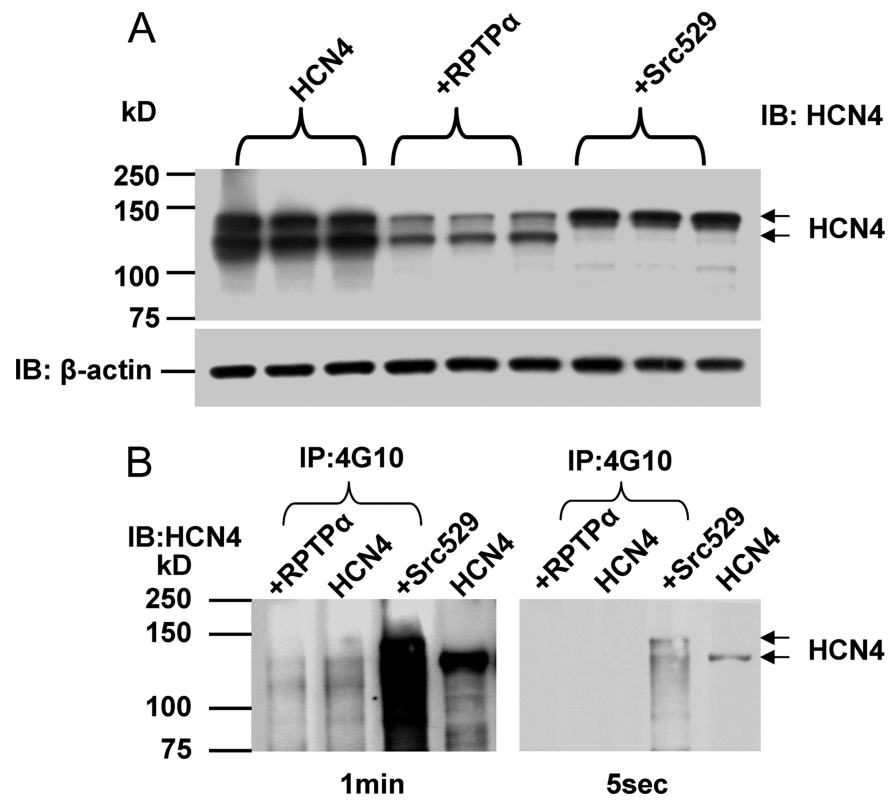

FIGURE 2. Src/RPTP $\alpha$ on HCN4 membrane protein expression and tyrosine phosphorylation in HEK293 cells. $A$, immunoblots (IB) on membrane fraction for HCN4, HCN4+ RPTP $\alpha$, and HCN4+SrC529. $\beta$-Actin was used as a loading control. $B$, tyrosine phosphorylation of HCN4 by Src529 and RPTP $\alpha$. The total protein samples were first immunoprecipitated (IP) using 4G10 antibody and then immunoblotted using an HCN4 antibody. Weak phosphorylation signals of HCN4 and HCN4+RPTP $\alpha$ can be seen in long exposed film (1 min). The immunoblot of HCN4 (right lane) was used as a size marker.

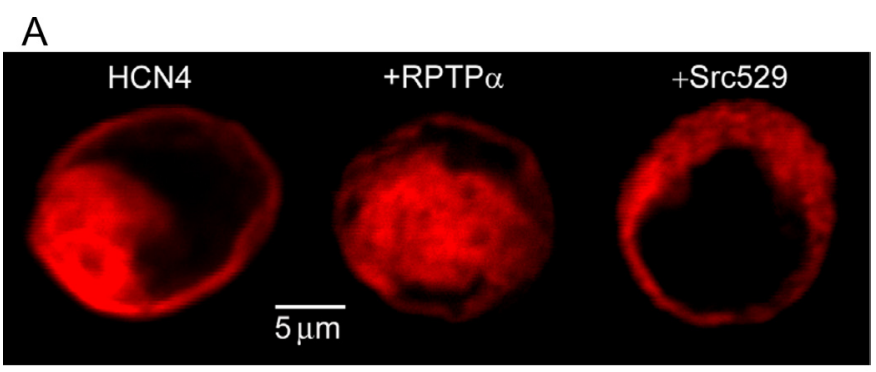

B

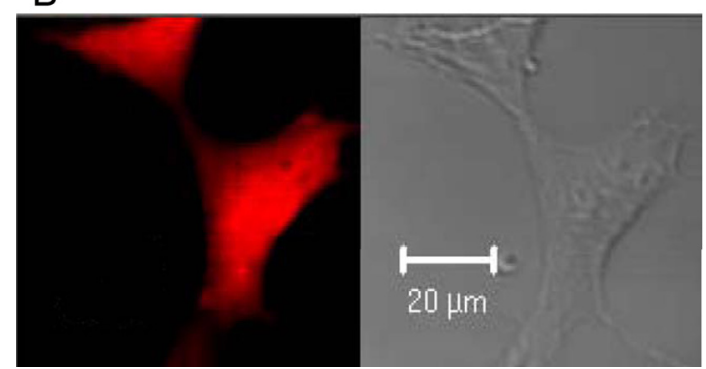

FIGURE 3. Src/RPTP $\alpha$ on HCN4 cell surface expression in HEK293 cells. $A$, fluorescence imaging of HCN4-DsRed, HCN4-DsRed+RPTP $\alpha$, and HCN4DsRed+Src529. B, fluorescence (left panel) and bright field (right panel) images of cells transfected with DsRed alone.

phosphorylation of the channel protein by Src tyrosine kinase and RPTP $\alpha$ tyrosine phosphatase, respectively.

To further seek supporting evidence that reduced ionic current expression of $\mathrm{HCN} 4$ is caused by the suppressed surface expression of $\mathrm{HCN} 4$ channels, we tagged $\mathrm{HCN} 4$ with a fluorescent protein, DsRed, and examined the distribution of $\mathrm{HCN} 4$ using fluorescent confocal microscopy. Fig. $3 A$ shows a typical fluorescent image of HCN4 expressed alone in a HEK293 cell 

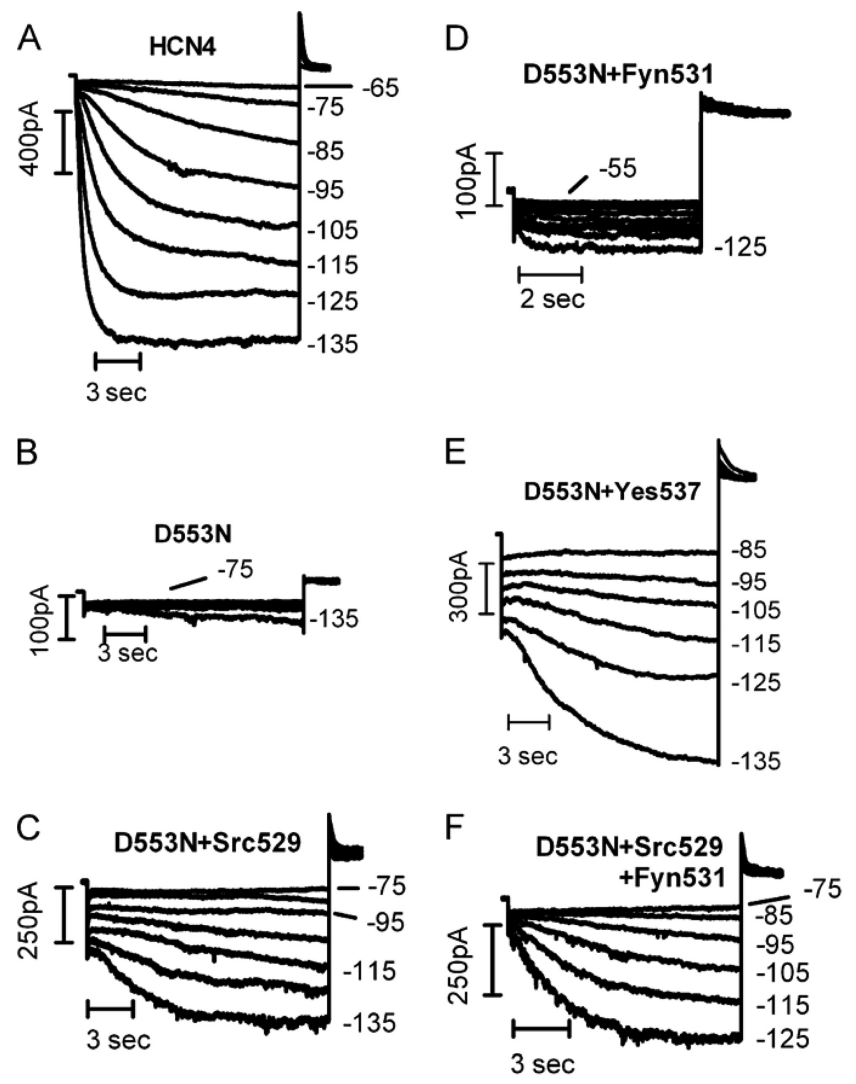

FIGURE 4. Src/Fyn/Yes kinases on current expression of D553N at $-125 \mathrm{mV}$. Currents elicited by hyperpolarizing pulses in 10-mV increments are presented for HCN4 (A), D553N (B), D553N+Src529 (C), D553N+Fyn531 (D), D553N+Yes537 (E)，D553N+Src529+Fyn531 (F)，D553N+Src529+Yes537 (G), $\mathrm{D} 553 \mathrm{~N}+\mathrm{Fyn} 531+$ Yes537 $(H)$, and D553N + Src529+Fyn531 + Yes537 (I). The test potentials are labeled in the figures. The pulse durations were $15 \mathrm{~s}$ for $A-C, E$, and $G ; 12 \mathrm{~s}$ for $F, H$, and $l$; and $6 \mathrm{~s}$ for $D$. The holding potential was $-10 \mathrm{mV}$.

(left panel). An approximately equal amount of HCN4 is distributed on the plasma membrane and in the cytosol, consistent with the membrane protein expression results (Fig. $2 A)$. When co-expressed with RPTP $\alpha$, most $\mathrm{HCN} 4$ channels are retained in the cytosol (middle panel of Fig. 3A). On the other hand, Src529 significantly enhanced the cell surface expression of HCN4 (Fig. $3 A$, right panel). As a control, Fig. $3 B$ shows the fluorescence (left panel) and bright field (right panel) images of the empty DsRed vector expressed in HEK293 cells.

Rescuing D553N Current Expression by Src, Fyn, and YesD553N has been recently identified in a patient suffering from sinus node dysfunction, long QT, ventricular tachycardia, and torsade de pointes (18). In vitro studies of the mutant channel revealed defective surface expression on plasma membrane, leading to the loss of current expression (18). Given the facts that the HCN4 channel activity including the channel surface expression can be significantly enhanced by Src-mediated tyrosine phosphorylation and the ubiquitous expression of three Src kinase family members (Src, Fyn, and Yes), we set forth to test the hypothesis that the current expression of the defective trafficking D553N can be restored by constitutively active forms of Src kinases (Src529, Fyn531, and Yes537).

Fig. 4 provides a typical set of current recordings under different conditions. The current expression of wild-type $\mathrm{HCN} 4$ is shown in Fig. $4 A$, as compared with the loss of current expres-
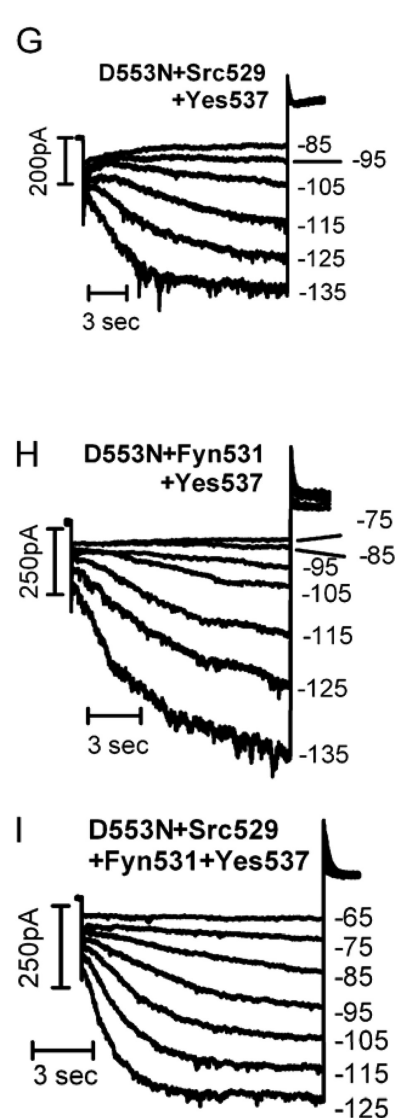

sion for D553N (Fig. 4B). Fig. 4 $(C-E)$ shows the effects of individual Src, Fyn, and Yes on D553N current expression, respectively. Fig. $4(F-H)$ also shows the effects of Src +Fyn, Src+Yes, and Fyn+Yes on D553N current expression, respectively. Fig. $4 I$ shows the overall effects of combined Src +Fyn+Yes on D553N current expression.

For effective comparison of the actions of Src kinases on D553N to HCN4, we calculated the current density and the activation kinetics at $-125 \mathrm{mV}$, which is near the fully activated voltage. Current density at the voltage in which all channels are open is directly related to our central interest of evaluating whether Src kinases can rescue the surface expression of D553N. The current densities under different conditions are shown in Fig. $5 \mathrm{~A}$.

Src and Yes, but not Fyn, can significantly rescue D553N current expression. Different combinations of three kinases all enhanced the current expression of D553N (Fig. $5 A$, asterisk). All three Src kinases expressed together (Fig. 4I) can restore $\sim 68 \%$ current expression of D553N as compared with the wildtype HCN4 expression (Fig. 5A, dark bars, HCN4: $37.15 \pm 3.21 \mathrm{pA} / \mathrm{pF}, n=7$; D553N+Src/Fyn/ Yes: $25.25 \pm 2.17 \mathrm{pA} / \mathrm{pF}, n=10$ ) (Fig. $5 A$ ).

The effects of Src/Fyn/Yes kinases on the current activation kinetics are also different (Fig. $5 B$ ). The time constants for activation kinetics were obtained by fitting the onset current with one-exponential function at $-125 \mathrm{mV}$ under different conditions. Fyn accelerated but Yes slowed the activation kinetics of D553N, whereas Src had no effects ( $p^{*}$ values). The combinations of Src+Fyn and Fyn + Yes can accelerate D553N activation kinetics, whereas the combination of Src +Yes cannot. A combination of all three Src kinases can speed D553N activation kinetics at $-125 \mathrm{mV}$ (Fig. $5, B$ and $D$ ); the time constants are $4.07 \pm 0.59 \mathrm{~s}(n=3)$ for D553N and $1.24 \pm 0.05 \mathrm{~s}(n=9)$ for $\mathrm{D} 553 \mathrm{~N} / \mathrm{Src} / \mathrm{Fyn} / \mathrm{Yes}$, respectively $(p=0.01)$. It is worth pointing out that in 16 D $553 \mathrm{~N}$ transfected cells we studied only three cells that expressed time-dependent inward currents at -125 $\mathrm{mV}$ and that were used for calculating current density and activation kinetics shown in Fig. $5(A$ and $B)$. The other 13 cells expressed no currents at the test potentials ranging from $-75 \mathrm{mV}$ to $-135 \mathrm{mV}$. As a comparison, we also showed the statistical analysis of comparing Src kinases on D553N to HCN4 indicated by $p$ values in Fig. $5 B$.

To assess the overall functional rescuing effects of Src/Fyn/ Yes on D553N gating, we examined the biophysical properties of D553N co-expressed with Src/Fyn/Yes (Fig. 4I) in compari- 
A
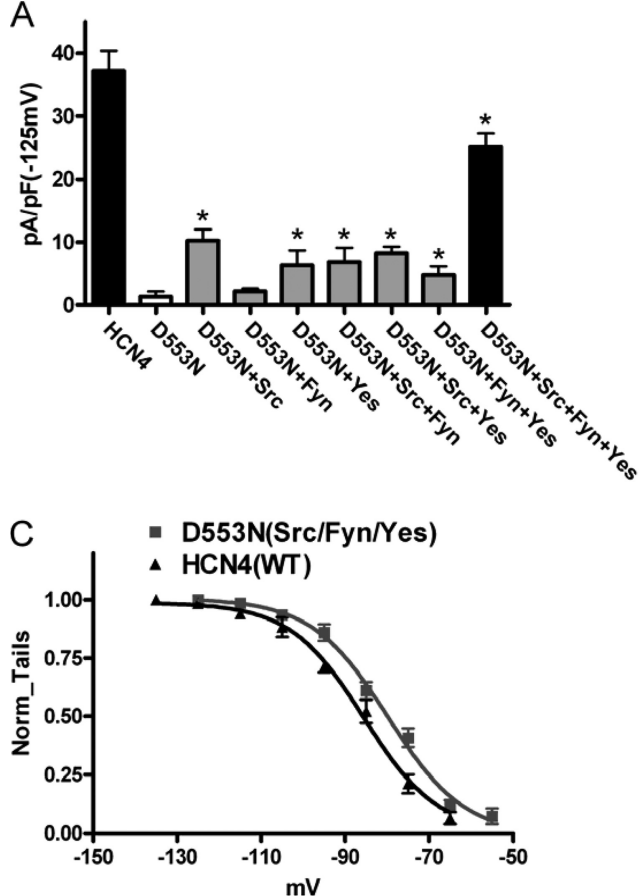
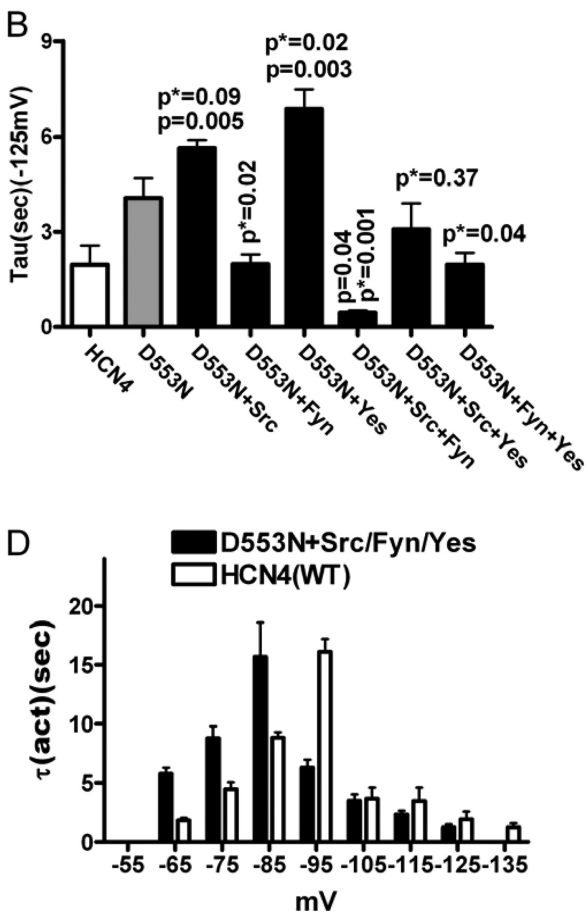

FIGURE 5. Src/Fyn/Yes kinases on current density and gating of D553N compared with HCN4. A, current density measured at $-125 \mathrm{mV}$ under different conditions. An asterisk indicates a statistically significant difference in comparison with D553N current density. B, time constants of activation kinetics at $-125 \mathrm{mV}$ under different conditions. $p$ values indicated statistical significance in comparing the effects of the kinases on D553N to HCN4 (white bar). $p^{*}$ values indicated statistical significance in comparing the effects of the kinases on D553N to D553N alone (gray bar). C, activation curves of HCN4 (WT) and D553N+Src/Fyn/Yes. D, activation kinetics of HCN4 (WT) and D553N+Src/Fyn/Yes. All of the statistical results were from seven to nine cells for each group except for D553N (only three cells expressed little currents of 16 cells tested).

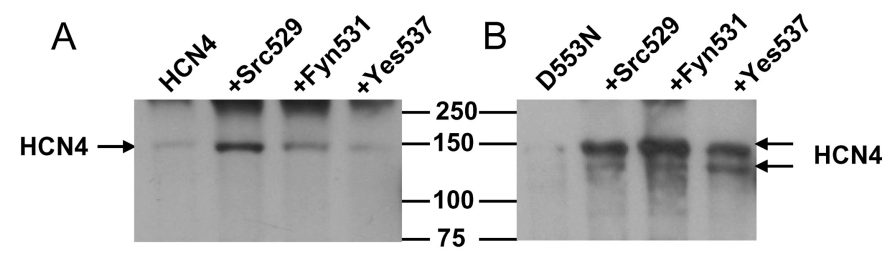

IP: 4G10

IB: HCN4

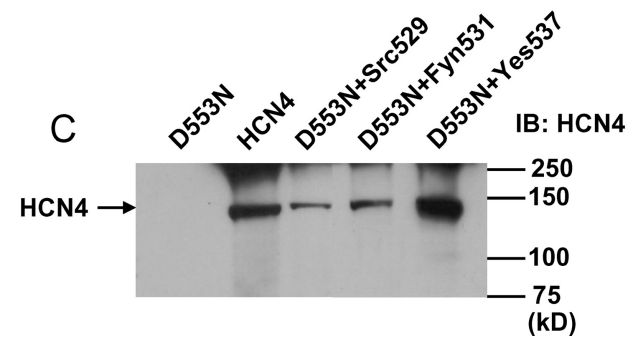

FIGURE 6. Src/Fyn/Yes kinases on tyrosine phosphorylation and surface expression of D553N. A, tyrosine phosphorylation of HCN4, HCN4+Src529, HCN4+Fyn531, and HCN4+Yes537, respectively. $B$, tyrosine phosphorylation of D553N, D553N+Src529, D553N+Fyn531, and D553N+Yes537, respectively. Samples for $A$ and $B$ were immunoprecipitated (IP) with 4G10 antibody prior to the detection by HCN4 antibody. C, Src/Fyn/Yes effects on surface expression of D553N using a biotinylation method. IB, immunoblot.

son with the wild-type HCN4 (Fig. 4A). The Boltzmann equation best fit from the averaged activation curves obtained from tail currents (see "Experimental Procedures" for details in the construction of the activation curve) showed a small depolarizing shift of D553N+Src/Fyn/Yes compared with HCN4 with no to depolarizing potentials (short term effect) and enhancing the cell surface expression (long term effect) $(12,14,15,17)$. We have now demonstrated that reduced tyrosine phosphorylation by RPTP $\alpha$ (Fig. $2 B$ ) can lead to the suppressed surface expression of HCN4 (Fig. 2A). To investigate whether tyrosine phosphorylation is involved in rescuing actions of Src, Fyn, and Yes on D553N current expression, we studied the tyrosine phosphorylation state of D553N by Src, Fyn, and Yes kinase, respectively. Fig. 6 shows the tyrosine phosphorylation of HCN4 (Fig. $6 A$ ) and D553N (Fig. 6B) by three Src kinases in HEK293 cells. $\mathrm{HCN} 4$ background phosphorylation was weak and significantly increased by Src529 and Fyn531, but not by Yes537. D553N background phosphorylation was barely detectable but dramatically increased by Src529, Fyn531, and Yes537. It was noticed that Src529 increased most significantly the phosphorylation of the wild-type $\mathrm{HCN} 4$, but Fyn531 induced the highest phosphorylation of D553N channel proteins.

We next examined the effects of Src kinases on D553N surface expression using biotinylation approach (see "Experimental Procedures" for details). Fig. $6 \mathrm{C}$ shows that the surface expression of D553N was barely detected. Src, Fyn, and Yes each significantly enhanced the surface expression to various degrees. Yes had a larger effect than Src and Fyn in promoting the surface expression of D553N. The HCN4 wild-type surface expression (Fig. 6C, second lane from the left) was used as a positive control.

To seek further supporting evidence for rescuing surface expression of D553N by three Src kinases, we constructed the 


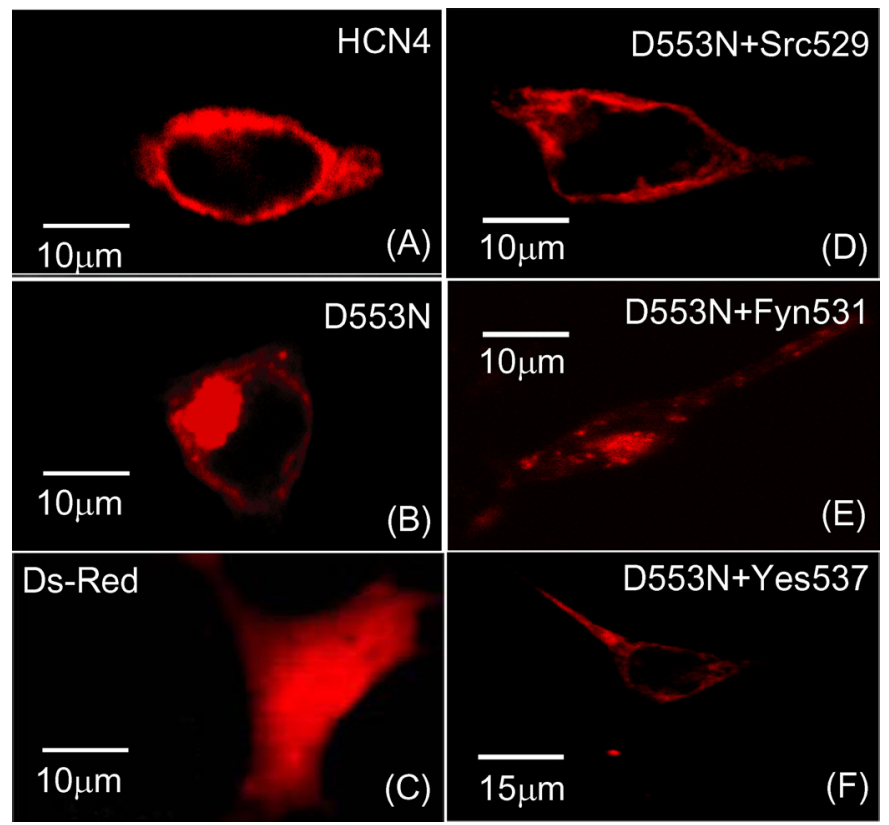

FIGURE 7. Src/Fyn/Yes kinases on D553N fluorescence imaging. Fluorescence images of cells transfected with HCN4 $(A)$, D553N (B), DsRed vector $(C)$, D553N+Src529 (D), D553N+Fyn531 (E), and D553N+Yes537 (F). All of the results were repeated in an additional eight to ten cells.

D553N-DsRed fusion protein and performed the confocal imaging experiments. Fig. 7 shows that most wild-type HCN4 is expressed on the cell surface (Fig. 7A). By contrast, most D553N cannot reach the cell surface (Fig. $7 B$ ). Co-expression with either Src (Fig. 7D) or Yes (Fig. 7F) can significantly enhance the surface expression of D553N. However, Fyn has been much less significant on D553N surface expression, as evidenced by a significant amount of D553N remaining in the cytosol (Fig. 7E). DsRed vector itself was uniformly expressed across the cell (Fig. $7 C$ ) and served as a negative control. These results, combined with the protein biochemistry analysis, collectively provide the cellular evidence for the functional rescue of D553N current expression at the plasma membrane by $\mathrm{Src} / \mathrm{Fyn} /$ Yes kinases.

\section{DISCUSSION}

Defective trafficking of mutant channels represents an important mechanism for Kv channels causing long QT2 (1). Studying long QT related Kv channel modulation has led to the findings that lower temperature and channel blockers can restore the surface and ionic current expression of the defective trafficking mutant channels (1). In this work, we showed for the first time that by modulating the Src/Fyn/Yes kinase activity, a human HCN4 trafficking defective mutant D553N (also linked to long QT (18)) can be rescued for normal surface and current expression. The corrected D553N exhibited the gating properties comparable with those of the wild-type HCN4 channels.

Enhanced tyrosine phosphorylation increased the activity of the cardiac pacemaker current, $I_{\mathrm{f}}$, in the sinoatrial node cells (13). Using genistein, a nonspecific tyrosine kinase inhibitor, we found a differential modulation of tyrosine phosphorylation for $\mathrm{HCN} 1, \mathrm{HCN} 2$, and HCN4 expressed in Xenopus oocytes; genistein had no effects on $\mathrm{HCN} 1$ but reduced $\mathrm{HCN} 2$ or HCN4 current expression (12). In the case of $\mathrm{HCN} 2$, there was also a negative shift in the voltage dependence of activation that accompanied the current reduction. These studies represent the acute effects of altered tyrosine phosphorylation of $\mathrm{HCN}$ channel proteins on the gating properties of $I_{\mathrm{f}}$.

To investigate the long term effects of specific tyrosine kinases on HCN channels in a mammalian background, we studied the effects of Src kinase on HCN4 channel (the main isoform in the heart) expressed in HEK293 cells. We focused on the Src kinases for two reasons: it mediates epidermal growth factor receptor signaling (19) and Src homology 3 domain was initially used to clone the first HCN channels (23). We found that Src associated with and phosphorylated the HCN4 channel proteins, leading to the enhanced HCN4 current density near diastolic potentials (15). This was the first direct evidence showing that 1) HCN4 channels can be phosphorylated by Srcmediated tyrosine kinases and 2) long term effects of tyrosine phosphorylation of $\mathrm{HCN} 4$ channels can induce the changes in the current density, which directly correlates with the number of functional channels expressed at the plasma membrane. Accompanying the increased current density were the accelerated activation kinetics and a positive shift in the voltage-dependent activation, which has been typically observed in the short term modulation of HCN4 channels. These conclusions were confirmed by the subsequent investigation on the major tyrosine residues that mediate Src actions (14). Using PP2, a selective inhibitor of Src kinase family, we found that the reduced Src kinase activity can indeed shift the voltage-dependent activation to hyperpolarizing potentials, an effect mediated by $\mathrm{HCN} 4 \mathrm{Tyr}^{531}$ (14). Another tyrosine residue, HCN4 $\mathrm{Tyr}^{554}$ previously reported by others (16), also contributed to the slowing of activation kinetics by PP2 (14). Work on the action of PP2 on $\mathrm{HCN} 4$ also resulted in two surprising observations. First, the PP2-induced negative shift of HCN4 voltage-dependent activation is not in agreement with our previous results with genistein. At least two factors can contribute to this discrepancy: mammalian cell (HEK293) versus amphibian (Xenopus oocytes) background and general (genistein) versus selective (PP2) inhibition of tyrosine kinases. Second, we found that PP2 also reduced the whole cell channel conductance (supplemental Fig. 3 in Ref. 14). These results implied that even the short term effect of altered tyrosine phosphorylation may affect the number of functional channels at the plasma membrane.

More recently in the investigation of the potential role the tyrosine phosphatase might play in the modulation of $\mathrm{HCN}$ channel function, we found the dramatic inhibition of $\mathrm{HCN} 2$ current expression by RPTP $\alpha$ (17). The inhibited HCN2 current expression was due to the reduced surface expression of $\mathrm{HCN} 2$ channels via association between RPTP $\alpha$ and the HCN2 channel proteins, resulting in the channel dephosphorylation (17). The work demonstrated a previously unrecognized feature of $\mathrm{HCN}$ channel modulation by tyrosine phosphorylation: the tyrosine phosphorylation state of $\mathrm{HCN}$ channel proteins represents one important regulatory mechanism for the cell surface expression of the functional channels, which directly determines the current expression of functional $\mathrm{HCN}$ channels. This feature may be utilized to enhance the surface and ionic current expression of $\mathrm{HCN}$ mutant channel that cannot reach the plasma membrane for normal function. 


\section{TABLE 1}

Relative roles of Src, Fyn, and Yes on D553N surface/current expression and activation kinetics

- , no or little expression or little effect or decrease; + , increase; ++ , significant increase or acceleration.

\begin{tabular}{cccc}
\hline & $\begin{array}{c}\text { Surface } \\
\text { expression }^{a}\end{array}$ & $\begin{array}{c}\text { Current } \\
\text { density }\end{array}$ & $\begin{array}{c}\text { Activation } \\
\text { kinetics }\end{array}$ \\
\hline D553N & - & - & \\
+ Src & ++ & ++ & - \\
+ Fyn & + & - & ++ \\
+ Yes & ++ & ++ & - \\
\hline
\end{tabular}

${ }^{a}$ Combined results of biotinylation and fluorescence imaging.

Indeed, the evidence presented in this work showed that the enhanced tyrosine phosphorylation mediated by Src kinases can rescue the surface expression of D553N for normal channel function. What was unexpected, however, is the finding that three Src kinases that were ubiquitously expressed in the heart have different functional effects on D553N channel activity. Subsequent phosphorylation studies showed that all three kinases significantly enhanced phosphorylation of D553N with the order of potency: Fyn531 > Src529 > Yes537 (Fig. 6B). In comparison, the wild-type channel background phosphorylation was increased mostly by Src529, to moderate degree by Fyn531, and nearly unaffected by Yes537. In agreement with the previous studies, the Src kinase-mediated tyrosine phosphorylation is associated with the acceleration of channel activation kinetics (14-16). These differential effects by $\mathrm{Src} / \mathrm{Fyn} /$ Yes on enhancing D553N expression and function are summarized in Table 1.

The differential phosphorylation of both wild-type $\mathrm{HCN} 4$ and D553N channels by three Src kinases suggested that different tyrosine residues are involved in mediating each of the kinases. These results also suggested a possibility that D553N may undergo a protein misfolding that prevents the nearby tyrosine residues from being phosphorylated. Association of Src tyrosine kinases appears to partially correct the protein folding that leads to the exposure of key tyrosine residues for phosphorylation.

To understand the mechanism by which tyrosine phosphorylation used to restore the surface expression of D553N, we proposed a model utilizing the three-dimensional crystal structure of the C-linker region of $\mathrm{HCN} 2$ for the following three reasons. First, D553N mutation occurred in the C-linker. Second, there is a high homology (91.6\%) between HCN2 and HCN4 in the C-linker (supplemental Fig. S1). Third, HCN2 is the only protein in the $\mathrm{HCN}$ family whose crystal structure of the C-linker has been solved (24). $\mathrm{HCN} 4 \mathrm{Asp}^{553}$ corresponds to $\mathrm{HCN} 2 \mathrm{Asp}^{475}$. Among many potential mechanisms responsible for the defective trafficking of HCN4-D553N, protein misfolding is an attractive one. We hypothesized that there may exist a potential electrostatic interaction between $\mathrm{Asp}^{475}$ and Lys ${ }^{472}$ of the $\mathrm{B}^{\prime}$ helix. The negatively charged $\mathrm{Asp}^{475}$ is spatially close to the positively charged Lys ${ }^{472}$, similar to the relative spatial locations of $\mathrm{Lys}^{472}$ and $\mathrm{Glu}^{502}$ of the $\mathrm{D}^{\prime}$ helix, which have been demonstrated to form a salt bridge critical in maintaining the local folding of C-linker (24) (supplemental Fig. S2). The putative D475N (equivalent to D553N in $\mathrm{HCN} 4$ ) mutation can cause the loss of a negative charge, which may change the intersubunit interaction between $\mathrm{Lys}^{472}$ and $\mathrm{Glu}^{502}$ to alter local folding of the C-linker structure. We noted that Lys $^{472}$ is changed to $\operatorname{Arg}^{550}$ in HCN4. With a guanyl group, $\operatorname{Arg}^{550}$ is more capable than Lys $^{472}$ in forming multiple electrostatic interactions with nearby residues having negative side chains. Therefore, in HCN4-D553N mutant, the potential salt bridge of Asp ${ }^{553}$ with $\mathrm{Arg}^{550}$ could be disrupted, which affected the interaction between $\operatorname{Arg}^{550}$ and $\mathrm{Glu}^{580}\left(\mathrm{Glu}^{502}\right.$ in $\left.\mathrm{HCN} 2\right)$ that is critical in intersubunit contacts (24). Furthermore, the Src kinase-mediated tyrosine phosphorylation at $\mathrm{Tyr}^{554}$ residue near $\mathrm{Asp}^{553}$ (supplemental Fig. S1) could introduce a negatively charged phosphate group, which could mimic the effect of $\mathrm{Asp}^{553}$ to rebuild the salt bridge interaction between $\operatorname{Arg}^{550}$ and $\mathrm{Glu}^{580}$, consistent with the previous studies demonstrating the importance of $\operatorname{Tyr}^{554}(14,16)$. For wild-type HCN4, the existing negative charge on $\mathrm{Asp}^{553}$ might repel the entry of a phosphate group and limit the phosphorylation on certain nearby tyrosine residues. It can explain why the differential modulation by Src, Fyn, and Yes is different between the wild-type HCN4 and the D533N mutant channels (Fig. 6, $A$ and $B$ ).

While presenting a novel mechanism to correct the surface expression of a trafficking defective HCN4 mutant channel, we left at least three questions unanswered. First, what are the tyrosine residue(s) in $\mathrm{HCN} 4$ channel proteins that mediate the actions of Fyn and Yes? The same tyrosine residues (such as $\mathrm{Tyr}^{531}$ and $\mathrm{Tyr}^{554}$ ) are unlikely to be used by all three kinases. Fyn, not Src, accelerated D553N activation kinetics (Fig. 5B). Fyn may target the tyrosine residues in or near $\mathrm{A}^{\prime}$ and $\mathrm{B}^{\prime}$ helices of the C-linker. On the other hand, Yes may phosphorylate different tyrosine residues that are located outside of the C-linker, which can explain its lack of acceleration in the activation kinetics. Second, what is the correlation, if any, among the surface expression and activation kinetics and the tyrosine phosphorylation state of HCN4 channels? Fyn showed significant tyrosine phosphorylation and acceleration of the channel activation kinetics but little effect on promoting the channel surface expression. Yes did not exert the highest phosphorylation in comparison with Src and Fyn (Fig. 6B), slowed the channel activation kinetics, but exhibited the most potency of promoting the cell surface expression of D553N (Figs. 6C and 7F and Table 1). It might involve other unknown proteins yet to be identified. Third, will an increase in the endogenous Src/Fyn/ Yes kinase activity in myocytes help promote the surface expression of D553N in vivo? Addressing these questions represents our future research endeavors, leading to the discovery of an effective endogenous regulatory mechanism to correct cardiac arrhythmias caused by HCN channel mutants.

Acknowledgments - We are grateful for the generous gift, RPTP $\alpha$, provided by Dr. Jan Sap. The constitutively active form of Fyn, Fyn531 in pcDNA3.1 vector, was kindly provided by Dr. Shigeru Kanda (Nagasaki University, Japan). The constitutively active form of Yes537 was kindly provided from Dr. Arkadiusz Welman (Edinburgh Cancer Research Center). We thank Dr. Qi Zhang for initial D553N construct.

\section{REFERENCES}

1. Delisle, B. P., Anson, B. D., Rajamani, S., and January, C. T. (2004) Circ. Res. 94, 1418-1428 
Rescue of a Trafficking Defective Pacemaker Channel

2. Zhou, Z., Gong, Q., Epstein, M. L., and January, C. T. (1998) J. Biol. Chem. 273, 21061-21066

3. Delisle, B. P., Anderson, C. L., Balijepalli, R. C., Anson, B. D., Kamp, T. J., and January, C. T. (2003) J. Biol. Chem. 278, 35749-35754

4. Zhou, Z., Gong, Q., and January, C. T. (1999) J. Biol. Chem. 274, 31123-31126

5. Furutani, M., Trudeau, M. C., Hagiwara, N., Seki, A., Gong, Q., Zhou, Z., Imamura, S., Nagashima, H., Kasanuki, H., Takao, A., Momma, K., January, C. T., Robertson, G. A., and Matsuoka, R. (1999) Circulation 99, 2290-2294

6. Paulussen, A., Raes, A., Matthijs, G., Snyders, D. J., Cohen, N., and Aerssens, J. (2002) J. Biol. Chem. 277, 48610-48616

7. Robinson, R. B., and Siegelbaum, S. A. (2003) Annu. Rev. Physiol. 65, 453-480

8. Yu, H., Wu, J., Potapova, I., Wymore, R. T., Holmes, B., Zuckerman, J., Pan, Z., Wang, H., Shi, W., Robinson, R. B., El-Maghrabi, M. R., Benjamin, W., Dixon, J., McKinnon, D., Cohen, I. S., and Wymore, R. (2001) Circ. Res. 88, E84-E87

9. Pian, P., Bucchi, A., Robinson, R. B., and Siegelbaum, S. A. (2006) J. Gen. Physiol. 128, 593-604

10. Fogle, K. J., Lyashchenko, A. K., Turbendian, H. K., and Tibbs, G. R. (2007) J. Neurosci. 27, 2802-2814

11. Poolos, N. P., Bullis, J. B., and Roth, M. K. (2006) J. Neurosci. 26, 7995-8003

12. Yu, H. G., Lu, Z., Pan, Z., and Cohen, I. S. (2004) Pflugers Arch. 447, 392-400

13. Wu, J. Y., Yu, H., and Cohen, I. S. (2000) Biochim. Biophys. Acta 1463,
$15-19$

14. Li, C. H., Zhang, Q., Teng, B., Mustafa, S. J., Huang, J. Y., and Yu, H. G. (2008) Am. J. Physiol. Cell Physiol. 294, C355-C362

15. Arinsburg, S. S., Cohen, I. S., and Yu, H. G. (2006) J. Cardiovasc. Pharmacol. 47, 578-586

16. Zong, X., Eckert, C., Yuan, H., Wahl-Schott, C., Abicht, H., Fang, L., Li, R., Mistrik, P., Gerstner, A., Much, B., Baumann, L., Michalakis, S., Zeng, R., Chen, Z., and Biel, M. (2005) J. Biol. Chem. 280, 34224-34232

17. Huang, J., Huang, A., Zhang, Q., Lin, Y. C., and Yu, H. G. (2008) J. Biol. Chem. 283, 29912-29919

18. Ueda, K., Nakamura, K., Hayashi, T., Inagaki, N., Takahashi, M., Arimura, T., Morita, H., Higashiuesato, Y., Hirano, Y., Yasunami, M., Takishita, S., Yamashina, A., Ohe, T., Sunamori, M., Hiraoka, M., and Kimura, A. (2004) J. Biol. Chem. 279, 27194-27198

19. Thomas, S. M., and Brugge, J. S. (1997) Annu. Rev. Cell Dev. Biol. 13, 513-609

20. Kalia, L. V., Gingrich, J. R., and Salter, M. W. (2004) Oncogene 23, 8007-8016

21. Barraclough, J., Hodgkinson, C., Hogg, A., Dive, C., and Welman, A. (2007) Neoplasia 9, 745-754

22. Kanda, S., Mochizuki, Y., Nakamura, T., Miyata, Y., Matsuyama, T., and Kanetake, H. (2005) J. Cell Sci. 118, 961-970

23. Santoro, B., Grant, S. G., Bartsch, D., and Kandel, E. R. (1997) Proc. Natl. Acad. Sci. U.S.A. 94, 14815-14820

24. Zagotta, W. N., Olivier, N. B., Black, K. D., Young, E. C., Olson, R., and Gouaux, E. (2003) Nature 425, 200-205 


\title{
Inactivation of L-type calcium channel modulated by $\mathrm{HCN} 2$ channel
}

\author{
Yen-Chang Lin, ${ }^{1,2}$ Jianying Huang, ${ }^{1,2}$ Qi Zhang, ${ }^{1,2}$ John M. Hollander, ${ }^{1,3}$ Jefferson C. Frisbee, ${ }^{1,2}$ \\ Karen H. Martin, ${ }^{4,5}$ Casey Nestor, ${ }^{2}$ Robert Goodman, ${ }^{2}$ and Han-Gang $\mathbf{Y u}^{1,2}$ \\ ${ }^{1}$ Center for Cardiovascular and Respiratory Sciences, ${ }^{2}$ Department of Physiology and Pharmacology, ${ }^{3}$ Department of \\ Exercise Physiology, ${ }^{4}$ Mary Babb Randolph Cancer Center, and ${ }^{5}$ Department of Neurobiology and Anatomy, West Virginia \\ University School of Medicine, Morgantown, West Virginia
}

Submitted 7 August 2009; accepted in final form 7 February 2010

Lin Y, Huang J, Zhang Q, Hollander JM, Frisbee JC, Martin KH, Nestor C, Goodman R, Yu H. Inactivation of L-type calcium channel modulated by HCN2 channel. Am J Physiol Cell Physiol 298: C1029-C1037, 2010. First published February 17, 2010; doi:10.1152/ajpcell.00355.2009.- $\mathrm{Ca}^{2+}$ entry is delicately controlled by inactivation of L-type calcium channel (LTCC) composed of the pore-forming subunit $\alpha 1 \mathrm{C}$ and the auxiliary subunits $\beta 1$ and $\alpha 2 \delta$. Calmodulin is the key protein that interacts with the $\mathrm{COOH}-$ terminal motifs of $\alpha 1 \mathrm{C}$, leading to the fine control of LTCC inactivation. In this study we show evidence that a hyperpolarizationactivated cyclic nucleotide-gated channel, $\mathrm{HCN} 2$, can act as a nonchannel regulatory protein to narrow the L-type $\mathrm{Ca}^{2+}$ channel currentvoltage curve. In the absence of LTCC auxiliary subunits, HCN2 can induce $\alpha 1 \mathrm{C}$ inactivation. Without $\alpha 2 \delta$, HCN2-induced fast inactivation of $\alpha 1 \mathrm{C}$ requires calmodulin. With $\alpha 2 \delta$, the $\alpha 1 \mathrm{C} / \mathrm{HCN} 2 / \alpha 2 \delta$ channel inactivation does not require calmodulin. In contrast, $\beta 1$ subunit plays a relatively minor role in the interaction of $\alpha 1 \mathrm{C}$ with $\mathrm{HCN} 2$. The $\mathrm{NH}_{2}$ terminus of $\mathrm{HCN} 2$ and the IQ motif of $\alpha 1 \mathrm{C}$ subunit are required for $\alpha 1 \mathrm{C} / \mathrm{HCN} 2$ channel interaction. $\mathrm{Ca}^{2+}$ channel inactivation is significantly slowed in hippocampus neurons (HNs) overexpressing $\mathrm{HCN} 2$ mutant lacking $\mathrm{NH}_{2}$ terminus and accelerated in HNs overexpressing the wild-type HCN2 compared with HN controls. Collectively, these results revealed a potentially novel protection mechanism for achieving the LTCC inactivation via interaction with $\mathrm{HCN} 2$.

L-type calcium channel inactivation; hyperpolarization-activated cyclic nucleotide-gated channels; calmodulin; hippocampal neuron

L-TYPE CALCIUM CHANNEL (LTCC) current inactivation is dependent on both $\mathrm{Ca}^{2+}$ ions and voltage $(3,13)$. The $\beta$ - and $\alpha 2 \delta$-subunits regulate the membrane expression and the voltage-dependent inactivation of the $\alpha 1 \mathrm{C}$-subunit (2). Calmodulin (CaM) plays a central role in both $\mathrm{Ca}^{2+}$-dependent inactivation (CDI) and voltage-dependent inactivation (VDI) using $\mathrm{Ba}^{2+}$ as the charge carrier $(12,13)$. CaM is proposed to tether with the IQ motif in the $\mathrm{COOH}$ terminus of $\alpha 1 \mathrm{C}(15,16) . \mathrm{Ca}^{2+}$ influx through LTCC activates apoCaM $\left(\mathrm{Ca}^{2+}\right.$-free $\left.\mathrm{CaM}\right) / \mathrm{IQ}$ complex. The activated CaM/IQ triggers a conformational change of the inactivation machinery consisting of $\alpha 1 \mathrm{C} \mathrm{COOH}$-terminal domains such as peptide A, peptide C (or CB), IQ motif, and $\mathrm{CaM}$. As a result, the inactivation machinery rapidly blocks the inner surface of the pore, leading to fast CDI $(6,16)$. $\mathrm{CaM}$ also has been proposed to interact with the linker of the transmembrane I and II segments associated with the $\beta 1$ subunit to modulate VDI $(3,12)$.

Address for reprint requests and other correspondence: H.-G. Yu, Center for Cardiovascular and Respiratory Sciences, West Virginia Univ. School of Medicine, One Medical Center Drive, Morgantown, WV 26506 (e-mail: hyu@hsc.wvu.edu).
Hyperpolarization-activated, cyclic nucleotide-gated (HCN) channels are activated by membrane hyperpolarization, generating a voltage- and time-dependent inward current called $I_{\mathrm{h}}$ or $I_{\mathrm{f}}$, which has been traditionally thought to permeate both $\mathrm{Na}^{+}$ and $\mathrm{K}^{+}$ions (4). Recently, we discovered that $\mathrm{Ca}^{2+}$ ions also can permeate $\mathrm{HCN}$ channels, even in a small percentage of the total current $(24,25)$. Furthermore, activation of $I_{\mathrm{h}}$ and $I_{\mathrm{f}}$ can increase the synapse transmission in dorsal root ganglion neurons (25) and shorten the action potential duration in ventricular myocytes (24), respectively. However, the underlying mechanisms are not well understood. In the present work, we focus on a hypothesis that a closed HCN channel can interact with LTCC at the plasma membrane, leading to the fast inactivation of L-type $\mathrm{Ca}^{2+}$ current $\left(I_{\mathrm{CaL}}\right)$.

\section{MATERIALS AND METHODS}

cDNA constructs. Various full-length cDNA plasmids were generous gifts from other investigators as detailed in the Supplemental Material. (Supplemental data for this article is available online at the American Journal of Physiology-Cell Physiology website.)

Cell culture and plasmids transfection. Human embryonic kidney (HEK)-293 cells were grown on poly-D-lysine-coated coverslips in Dulbecco's modified Eagle's medium (Invitrogen) supplemented with $10 \%$ fetal bovine serum, $100 \mathrm{IU} / \mathrm{ml}$ penicillin, and $100 \mathrm{~g} / \mathrm{l}$ streptomycin. Cells with $50-70 \%$ confluence in a six-well plate were used for plasmid transfection (2-5 $\mu \mathrm{g}$ for each plasmid) using Lipofectamine2000 (Invitrogen).

An HEK-293 cell line stably expressing $\alpha 2 \delta / \beta 1$ was kindly provided by Dr. Richard Tsien (Stanford University). Culture of these cells was similar to that described above except for addition of antibiotics, G418 $(0.5 \mathrm{mM} / \mathrm{ml})$ and $5 \mathrm{mM} \mathrm{L}$-glutamine, to the DMEM.

Preparation of hippocampus tissue. Adult rats (300-350 g) were euthanized by intraperitoneal injection of pentobarbital sodium (1.2 $\mathrm{g} / \mathrm{kg}$ ). After euthanasia, the adult rats were decapitated. The protocol was approved by the Institutional Animal Care and Use Committee of the Health Sciences Center at West Virginia University. Hippocampal tissues were cut out and quickly frozen in liquid nitrogen.

Cell lysis, immunoprecipitation, and Western blot analysis. Coimmunoprecipitation and Western blot experiments were the same as previously reported (11). Details are provided in the Supplemental Material.

Hippocampus neuron culture, transfection, and imaging. Primary embryonic day 18 rat hippocampus neurons were used for studying the interaction between $\alpha 1 \mathrm{C}$ and $\mathrm{HCN} 2$. Neuron culture and plasmid transfection were completed following the instruction manual (Invitrogen). Live-cell images of hippocampal neurons were visualized using an inverted Nikon TE2000S epifluorescent microscope with Prior filter wheels and a Photometrics Coolsnap HQ charge-coupled device camera. More details are provided in the Supplemental Material.

Whole cell patch-clamp recordings. Patch-clamp recordings are the same as previously reported (11). The pipettes had a resistance of 2-5 $\mathrm{M} \Omega$ when filled (in $\mathrm{mM}$ ): $108 \mathrm{CsCl}, 4 \mathrm{MgCl}_{2}, 9$ EGTA, and 9 
HEPES. The bath solution contained (in mM) $2 \mathrm{BaCl}_{2}, 1 \mathrm{MgCl}_{2}, 10$ HEPES, 40 tetraethylammonium, 10 glucoses, and $65 \mathrm{CsCl}$. Both solutions were adjusted with $\mathrm{CsOH}$ to $\mathrm{pH}$ 7.2. Unless specifically mentioned in the text, the $\mathrm{Ba}^{2+}$ currents $\left(I_{\mathrm{Ba}}\right)$ were recorded at +10 $\mathrm{mV}$ from a holding potential of $-90 \mathrm{mV}$. $I_{\mathrm{Ba}}$ current inactivation was sometimes normalized for better comparison of kinetics in different combinations of plasmids coexpressed in different HEK-293 cells (e.g., Figs. 2, $A$ and $D$, and $3, A-C$ ). Normalization is indicated without vertical scales; where vertical scales are present, $\mathrm{P} / 4$ protocols were used to remove the leak currents. The capacitive currents were minimized with $75-85 \%$ series resistance compensation. More details are provided in the Supplemental Material.

\section{RESULTS}

$C D I$ and VDI of $\alpha 1 C / \alpha 2 \delta / \beta 1$ and $\alpha 1 C / \alpha 2 \delta / \beta 1 / H C N 2$ channels in HEK-293 cells. Figure $1 A$ exemplified a well-established difference between CDI and VDI: faster inactivation for $I_{\mathrm{Ca}}$ (solid line) than for $I_{\mathrm{Ba}}$ (shaded line), recorded at $+10 \mathrm{mV}$. This difference, however, is largely diminished in the presence of HCN2 (Fig. $1 B$ ): like $I_{\mathrm{Ca}}$, the $I_{\mathrm{Ba}}$ of $\alpha 1 \mathrm{C} / \alpha 2 \delta /$ $\beta 1 / \mathrm{HCN} 2$ can inactivate almost completely to the closed state. Figure $1 C$ summarizes the time constants of inactivation of $I_{\mathrm{Ca}}$ and $I_{\mathrm{Ba}}$ for $\alpha 1 \mathrm{C} / \alpha 2 \delta / \beta 1$ and $\alpha 1 \mathrm{C} / \alpha 2 \delta / \beta 1 / \mathrm{HCN} 2$. They are $25.0 \pm 3.2 \mathrm{~ms}$ for $I_{\mathrm{Ca}}$ and $228.5 \pm 24.2 \mathrm{~ms}$ for $I_{\mathrm{Ba}}$ of $\alpha 1 \mathrm{C} / \alpha 2 \delta / \beta 1$, and $58.3 \pm 3.3 \mathrm{~ms}$ for $I_{\mathrm{Ca}}$ and $72.5 \pm 5.6 \mathrm{~ms}$ for $I_{\mathrm{Ba}}$ of $\alpha 1 \mathrm{C} / \alpha 2 \delta / \beta 1 / \mathrm{HCN} 2$. The difference between $I_{\mathrm{Ca}}$ and $I_{\mathrm{Ba}}$ of $\alpha 1 \mathrm{C} / \alpha 2 \delta / \beta 1 / \mathrm{HCN} 2$ inactivation kinetics was diminished compared with that between $I_{\mathrm{Ca}}$ and $I_{\mathrm{Ba}}$ of $\alpha 1 \mathrm{C} / \alpha 2 \delta / \beta 1$ inactivation kinetics, indicating that $\mathrm{HCN} 2$ can help complete the $I_{\mathrm{Ba}}$ inactivation.

We also examined whether the current-voltage relationship curves of $\alpha 1 \mathrm{C} / \alpha 2 \delta / \beta 1$ are altered by $\mathrm{HCN} 2$. Representative $I_{\mathrm{Ca}}$ are shown for $\alpha 1 C / \alpha 2 \delta / \beta 1$ (Fig. $1 D$ ) and $\alpha 1 C / \alpha 2 \delta / \beta 1 / \mathrm{HCN} 2$ (Fig. 1E). In the presence of HCN2, there was a positive shift in the threshold activation of $I_{\mathrm{CaL}}$ and more reduced current amplitude at the depolarized end of the bell shape near +50 $\mathrm{mV}$ (Fig. 1F, shaded line). All results were repeated in an additional five to eight cells.

Fast inactivation of $\alpha 1 C / H C N 2$ channels in HEK-293. To examine a possible hidden interaction between $\alpha 1 \mathrm{C}$ and $\mathrm{HCN} 2$, we studied $\alpha 1 \mathrm{C}$ inactivation by coexpressing $\alpha 1 \mathrm{C}$ and $\mathrm{HCN} 2$ in the absence of auxiliary subunits. With the use of $\mathrm{Ba}^{2+}$ as the charge carrier, $\alpha 1 \mathrm{C}$ exhibited little current inactivation when expressed alone (Fig. 2A, top). The auxiliary subunit $\alpha 2 \delta$ could limit the $\mathrm{Ca}^{2+}$ influx by inducing a slow inactivation of $\alpha 1 \mathrm{C}$ (Fig. 2A, top). Figure 2B, bottom, shows the absence of endogenous time-dependent inward currents in HEK-293 cells without cDNA transfection under our experimental conditions. These results are in agreement with the previous reports $(7,23)$. However, when HCN2 was coexpressed with $\alpha 1 \mathrm{C}$, a fast inactivation of $\alpha 1 \mathrm{C} / \mathrm{HCN} 2$ was observed (Fig. 2B,top). This new inactivation has a differential sensitivity for $\mathrm{Ba}^{2+}$ and $\mathrm{Ca}^{2+}$ ions, capable of coming back to the closed state in the presence of $\mathrm{Ca}^{2+}$ (shaded line) but not $\mathrm{Ba}^{2+}$ (solid line), the typical characteristics of the conventional LTCC inactivation $(12,28)$. Adding $\alpha 2 \delta$ did not significantly affect the $\alpha 1 \mathrm{C} / \mathrm{HCN} 2$ inactivation (Fig. 2A, bottom). With $\mathrm{Ba}^{2+}$ as the charge carrier, time constants for inactivation of $\alpha 1 \mathrm{C} / \alpha 2 \delta, \alpha 1 \mathrm{C} / \mathrm{HCN} 2$, and $\alpha 1 \mathrm{C} / \mathrm{HCN} 2 / \alpha 2 \delta$ were $156 \pm 19,26 \pm 4$, and $22 \pm 2 \mathrm{~ms}$, respectively $(n=8)$. The inactivation for $\alpha 1 \mathrm{C} / \alpha 2 \delta$ channels was significantly slower than that for $\alpha 1 \mathrm{C} /$ $\mathrm{HCN} 2$ or $\alpha 1 \mathrm{C} / \mathrm{HCN} 2 / \alpha 2 \delta(P=0.002, n=8)$. There was no statistical difference between $\alpha 1 \mathrm{C} / \mathrm{HCN} 2$ and $\alpha 1 \mathrm{C} / \mathrm{HCN} 2 / \alpha 2 \delta$
A

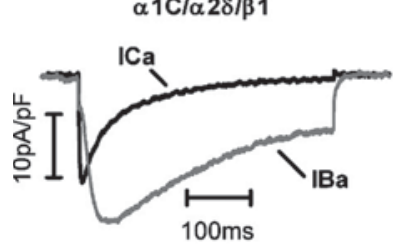

D

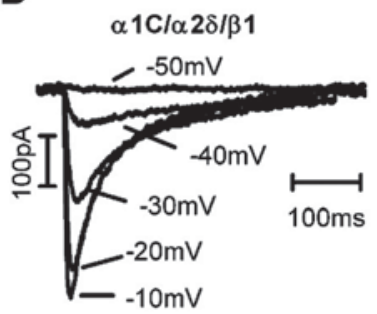

B

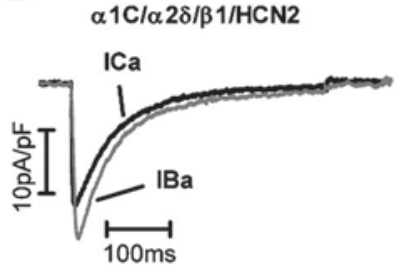

E

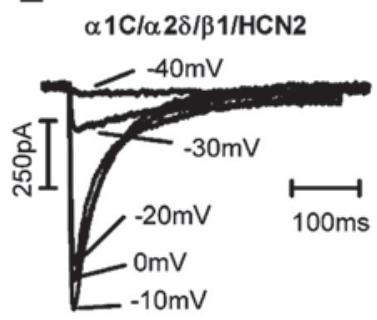

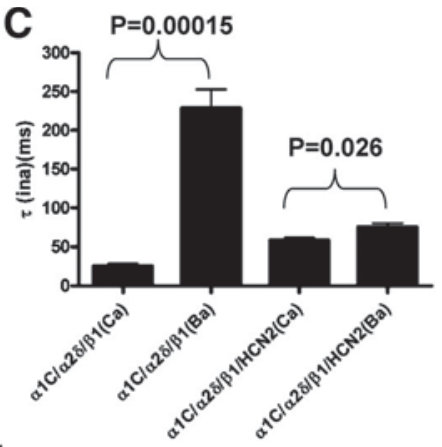

$\mathbf{F}$

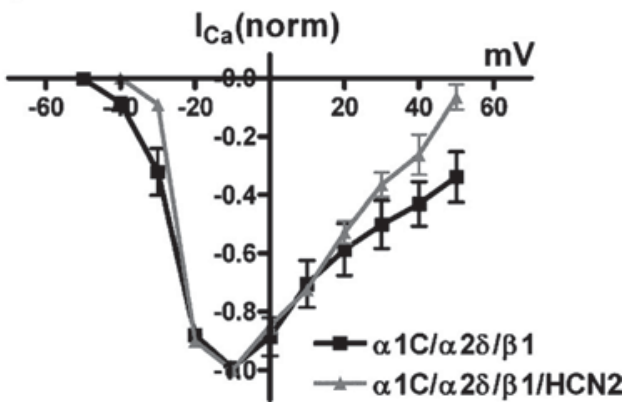

Fig. 1. $\mathrm{Ca}^{2+}$-dependent inactivation (CDI)- and voltage-dependent inactivation (VDI) of $\alpha 1 \mathrm{C} / \alpha 2 \delta / \beta 1$ and $\alpha 1 \mathrm{C} / \alpha 2 \delta / \beta 1 / \mathrm{HCN} 2$ in $\mathrm{HEK}-293$. $A$ : Ca ${ }^{2+}$ current $\left(I_{\mathrm{Ca}}\right.$; solid line) and $\mathrm{Ba}^{2+}$ current $\left(I_{\mathrm{Ba}}\right.$; shaded line) of $\alpha 1 \mathrm{C} / \alpha 2 \delta / \beta 1$ recorded at $+10 \mathrm{mV}$. B: $I_{\mathrm{Ca}}$ (solid line) and $I_{\mathrm{Ba}}($ shaded line) of $\alpha 1 \mathrm{C} / \alpha 2 \delta / \beta 1 / \mathrm{HCN} 2 \mathrm{recorded}$ at $+10 \mathrm{mV}$. $C$ : time constants $(\tau)$ for inactivation of $I_{\mathrm{Ca}}$ and $I_{\mathrm{Ba}}$ at $+10 \mathrm{mV}$ for $\alpha 1 \mathrm{C} / \alpha 2 \delta / \beta 1$ and $\alpha 1 \mathrm{C} / \alpha 2 \delta / \beta 1 / \mathrm{HCN} 2$, respectively. For simplicity of comparison, we best fit the currents with one exponential function. $P$ values shown are the statistical results of comparing $I_{\mathrm{Ca}}$ and $I_{\mathrm{Ba}}$ inactivation. $D$ and $E$ : $I_{\mathrm{Ca}}$ currents recorded in the test pulses ranging from -50 to $+50 \mathrm{mV}$ in $10-\mathrm{mV}$ increments for $\alpha 1 \mathrm{C} / \alpha 2 \delta / \beta 1(D)$ and $\alpha 1 \mathrm{C} / \alpha 2 \delta / \beta 1 / \mathrm{HCN} 2(E)$, respectively. A $400-\mathrm{ms}$ depolarizing pulse was used. Holding potential was $-90 \mathrm{mV} . F$ : current-voltage relationship curves for $\alpha 1 \mathrm{C} / \alpha 2 \delta / \beta 1$ and $\alpha 1 \mathrm{C} / \alpha 2 \delta / \beta 1 / \mathrm{HCN} 2$. 
A
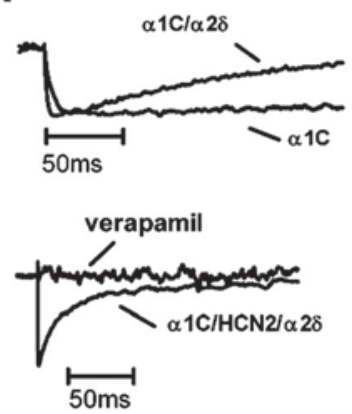

C HCN2 (-125mV)

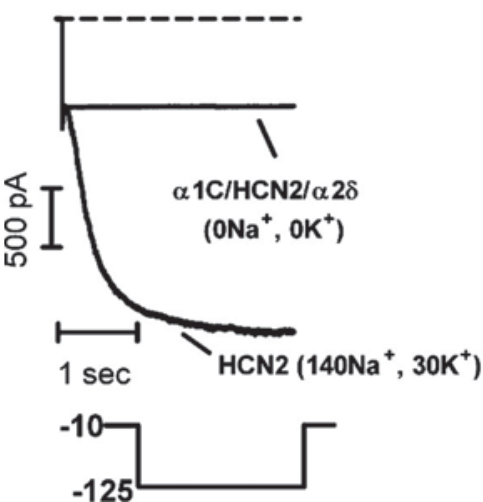

B $\quad \alpha$ 1C/HCN2
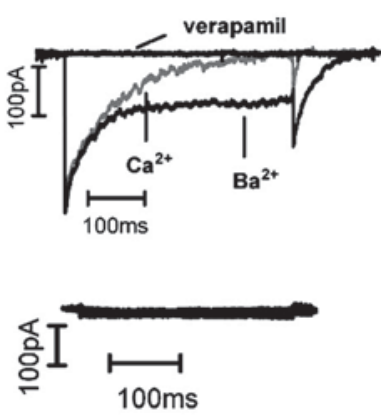

D "I $\mathrm{Ca} "(+10 \mathrm{mV})$
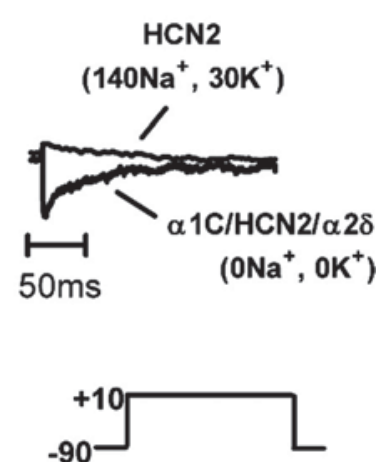

Fig. 2. $\alpha 1 \mathrm{C} / \mathrm{HCN} 2$ channel inactivation in HEK-293 cells. A, top: current expression of $\alpha 1 \mathrm{C}$ and $\alpha 1 \mathrm{C} / \alpha 2 \delta$, respectively. Bottom, current inactivation of $\alpha 1 \mathrm{C} / \mathrm{HCN} 2 / \alpha 2 \delta$ that was blocked by $10 \mu \mathrm{M}$ verapamil. Pulse duration was $200 \mathrm{~ms}$. $B$, top: $\alpha 1 \mathrm{C} / \mathrm{HCN} 2$ inactivation using $2 \mathrm{mM} \mathrm{Ba}^{2+}$ (solid line) or $2 \mathrm{mM}$ $\mathrm{Ca}^{2+}$ (shaded line) as the charge carrier, which was blocked by $10 \mu \mathrm{M}$ verapamil. Pulse duration was $400 \mathrm{~ms}$. Bottom, absence of time-dependent inward current expressions in HEK-293 cells without plasmid transfection. Pulse duration was $300 \mathrm{~ms}$. In $A$, the current inactivation was normalized for better comparison of the inactivation kinetics. $C$ : hyperpolarizing pulse control experiments. In cells expressing only $\mathrm{HCN} 2, I_{\mathrm{HCN} 2}$ was elicited by a 3 -s hyperpolarizing pulse to $-125 \mathrm{mV}$ from the holding potential of $-10 \mathrm{mV}$ in modified Tyrode solution containing $140 \mathrm{mM} \mathrm{Na}^{+}$and $30 \mathrm{mM} \mathrm{K}^{+}$; in cells coexpressing $\alpha 1 \mathrm{C} / \mathrm{HCN} 2 / \alpha 2 \delta$, the same hyperpolarizing pulse could not detect $I_{\mathrm{HCN} 2}$ in the external and internal solutions used to record $I_{\mathrm{Ba}}$. Although the leak current varies from cell to cell, we chose a leak current that closely aligned with the HCN2 time-dependent inward current for better comparison. The pulse protocol is shown in the inset. The dashed line shows the holding current $D$ : depolarizing pulse control experiments. In solutions without $\mathrm{Na}^{+}$and $\mathrm{K}^{+}$ ions $\left(0 \mathrm{Na}^{+}, 0 \mathrm{~K}^{+}\right), I_{\mathrm{Ba}}$ was recorded only in cells coexpressing $\alpha 1 \mathrm{C} / \mathrm{HCN} 2 /$ $\alpha 2 \delta$, not in cells expressing only HCN2. The pulse protocol is shown in the inset.

channel inactivation $(P=0.5, n=8)$. To verify that the current is via the conventional $\alpha 1 \mathrm{C}$ channel, verapamil $(10$ $\mu \mathrm{M}$ ) was applied to block the current activation (Fig. 2, A, bottom, and $B$, top).

To address the concern that the fast inactivation of $\alpha 1 \mathrm{C} /$ $\mathrm{HCN} 2$ or $\alpha 1 \mathrm{C} / \mathrm{HCN} 2 / \alpha 2 \delta$ channel was probably due to the contamination from the deactivation of HCN2 channels, we studied current expression of $\mathrm{HCN} 2$ in the external solution normally used for characterization of $\mathrm{HCN} 2$ and in the external solution used to record $I_{\mathrm{Ba}}$ inactivation (e.g., Fig. 2, $A$ and $B$ ). The hyperpolarization-activated $\mathrm{HCN} 2$ channels permeate both $\mathrm{Na}^{+}$and $\mathrm{K}^{+}$ions (4). The channel cannot be activated in the absence of external $\mathrm{Na}^{+}$and $\mathrm{K}^{+}$ions (4). In the external solution containing $140 \mathrm{mM} \mathrm{Na}^{+}$and $30 \mathrm{mM} \mathrm{K}^{+}$, a typical

HCN2 current (the time-dependent inward current) was recorded in response to a 3-s hyperpolarizing pulse to $-125 \mathrm{mV}$ (Fig. 2C), consistent with the previous reports (e.g., Ref. 24). When the $\mathrm{Na}^{+}$and $\mathrm{K}^{+}$ions were removed from the external solution, no time-dependent inward current was recorded (Fig. 2C).

HCN2 channels are closed at membrane depolarization that is used to activate LTCC (4). In response to a depolarization to $+10 \mathrm{mV}$ from the holding potential of $-90 \mathrm{mV}$, there was no HCN2 activation current in the solution containing $140 \mathrm{mM}$ $\mathrm{Na}^{+}$and $30 \mathrm{mM} \mathrm{K}^{+}$(Fig. 2D). The inactivation of $\alpha 1 \mathrm{C} /$ $\mathrm{HCN} 2 / \alpha 2 \delta$ channel in the $\mathrm{Na}^{+}$- and $\mathrm{K}^{+}$-free solution was used as a comparison (Fig. 2D). These data strongly indicate that the closed HCN2 channels can act as the nonfunction channel to induce a fast inactivation of $\alpha 1 \mathrm{C}$.

Role of $\mathrm{Ca}^{2+}$-free $\mathrm{CaM}$ in $\alpha 1 \mathrm{C} / \mathrm{HCN} 2$ channel inactivation. Given the pivotal role CaM plays in the $\mathrm{Ca}^{2+}$ channel inactivation, we tested the idea that CaM may be involved in the fast inactivation of $\alpha 1 \mathrm{C} / \mathrm{HCN} 2$ channel for two reasons: $\mathrm{CaM}$ is endogenously present in HEK-293 cells, and apoCaM (no $\mathrm{Ca}^{2+}$-bound $\mathrm{CaM}$ ) is tethered to structural domains in the $\mathrm{COOH}$ terminus of $\alpha 1 \mathrm{C}$ subunit $(6,16)$.

We first used two structurally different calmodulin inhibitors, W-7 [N-(6-aminohexyl)-5-chloro-1-naphthalenesulfonamide] (10) and trifluoperazine (TFP) (18), to study their potential effects on $\alpha 1 \mathrm{C} / \mathrm{HCN} 2$ channel inactivation. After binding $\mathrm{Ca}^{2+}$ ions, CaM undergoes a conformational change leading to the exposure of its hydrophobic activation site for binding to its substrate, CaM-dependent kinase (CaMK). Both W-7 and TFP block CaM actions by occupying the hydrophobic site to prevent binding of CaMK $(10,14)$. Figure $3 A$ shows that both drugs at $15 \mu \mathrm{M}$ eliminated the fast inactivation of $\alpha 1 \mathrm{C} / \mathrm{HCN} 2$ channels within 15-20 min, consistent with the time course of inhibiting $\mathrm{CaM}$ activity by both drugs. The effect of the drug was observed in the same cell. The similar results were obtained in an additional five to seven cells.

We then employed a dominant negative calmodulin mutant, $\mathrm{CaM}_{(1,2,3,4)}$ (22). Compared with the current expression of $\alpha 1 \mathrm{C} / \mathrm{HCN} 2$ coexpressed with a wild-type calmodulin, $\mathrm{CaM}_{(\mathrm{WT})}$, overexpression of $\mathrm{CaM}_{(1,2,3,4)}$ eliminated the fast inactivation of $\alpha 1 \mathrm{C} / \mathrm{HCN} 2$ (Fig. 3B, shaded line). These pharmacological and mutant $\mathrm{CaM}$ data strongly suggested that the interaction of $\alpha 1 \mathrm{C}$ and $\mathrm{HCN} 2$ is partially mediated by CaM.

Role of $\alpha 2 \delta$ in $\alpha 1 C / H C N 2$ channel inactivation. Nearly all studies of CaM-mediated $\alpha 1 \mathrm{C}$ inactivation, carried out in either Xenopus oocytes or mammalian cells (HEK-293, Chinese hamster ovary, COS), have been performed with coexpression of $\beta 1$ - and $\alpha 2 \delta$-subunits. This is due to the important roles of $\beta 1$ - and $\alpha 2 \delta$-subunits in expression of $\alpha 1 \mathrm{C}$ on plasma membrane and $\mathrm{Ca}^{2+}$ channel function (2). Previous studies also have demonstrated a significant role of $\alpha 2 \delta$ in initiating the inactivation of $\alpha 1 \mathrm{C}$ expressed in HEK-293 cells $(7,23)$. Wondering whether $\mathrm{CaM}$ would have the similar impact on the $\alpha 1 \mathrm{C} / \mathrm{HCN} 2$ channel inactivation in the presence of the $\alpha 2 \delta$ subunit, we tested the effects of W-7 and TFP on the current expression of $\alpha 1 \mathrm{C} / \mathrm{HCN} 2 / \alpha 2 \delta$ channels. Surprisingly, neither W-7 (left) nor TFP (right) affected the inactivation of $\alpha 1 \mathrm{C} /$ HCN $2 / \alpha 2 \delta$ channels (Fig. $3 C$, shaded lines). To further confirm this observation, we overexpressed either $\mathrm{CaM}_{(\mathrm{WT})}$ or $\mathrm{CaM}_{(1,2,3,4)}$ with $\alpha 1 \mathrm{C} / \mathrm{HCN} 2 / \alpha 2 \delta$ channels (Fig. 3D, shaded lines). Neither of them affected the inactivation of $\alpha 1 \mathrm{C} / \mathrm{HCN} 2 /$ $\alpha 2 \delta$ channels. Compared with its dominant effect in $\alpha 1 \mathrm{C} /$ 
Fig. 3. Role of calmodulin $(\mathrm{CaM})$ in $\alpha 1 \mathrm{C} /$ $\mathrm{HCN} 2$ channel inactivation. $A: \alpha 1 \mathrm{C} / \mathrm{HCN} 2$ channel inactivation (solid line) and lost inactivation in the presence of the $\mathrm{CaM}$ inhibitor W-7 or trifluoperazine (TFP) (shaded lines). $B: \alpha 1 \mathrm{C} / \mathrm{HCN} 2 / \mathrm{CaM}_{(\mathrm{WT})}$ channel inactivation (solid line) and lost inactivation of $\alpha 1 \mathrm{C} / \mathrm{HCN} 2 /$ $\mathrm{CaM}_{(1,2,3,4)}$ channels (shaded line). $\mathrm{CaM}(\mathrm{WT})$, wild-type calmodulin; $\mathrm{CaM}_{(1,2,3,4)}$, a dominant negative calmodulin mutant. $C: \alpha 1 \mathrm{C} / \mathrm{HCN} 2 /$ $\alpha 2 \delta$ channel inactivation in the absence (solid line) and presence of W-7 (shaded line; left) or TFP (shaded line; right). $D: \alpha 1 \mathrm{C} / \mathrm{HCN} 2 / \alpha 2 \delta$ channel inactivation in the absence (solid line) and presence of $\mathrm{CaM}_{(1,2,3,4)}$ (shaded line).
A
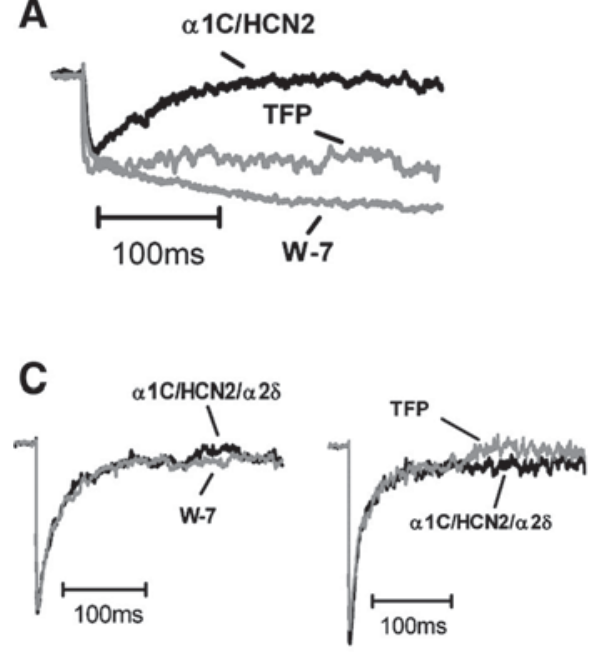

B
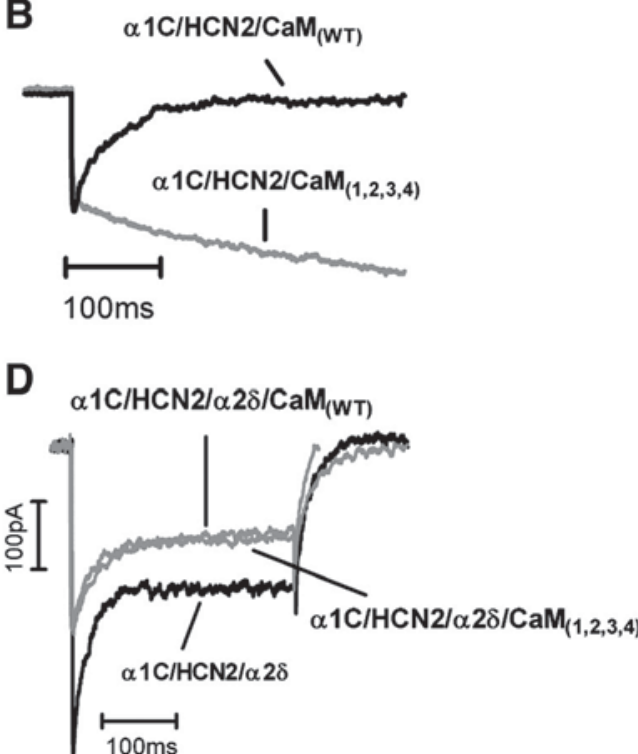

HCN2 channel inactivation (Fig. $3, A$ and $B$ ), CaM plays a minor role in $\alpha 1 \mathrm{C} / \mathrm{HCN} 2 / \alpha 2 \delta$ channel inactivation (Figs. 3, $C$ and $D$ ), indicating a potential role of $\alpha 2 \delta$-subunit in the $\alpha 1 \mathrm{C} / \mathrm{HCN} 2$ channel inactivation.

$\alpha 1 C / H C N 2$ channel interaction requires $\mathrm{NH}_{2}$ terminus of $H C N 2$ and $I Q$ motif of $\alpha I C$. The fact that the fast inactivation of $\alpha 1 \mathrm{C} / \mathrm{HCN} 2$ is $\mathrm{Ca}^{2+}$ independent (since we used $\mathrm{Ba}^{2+}$ as a charge carrier) suggested that $\mathrm{CaM}$ may not be the only mechanism for $\alpha 1 \mathrm{C} / \mathrm{HCN} 2$ channel interaction. To explore an unconventional idea that HCN2 may also interact with $\alpha 1 \mathrm{C}$, we studied the current expression of 1 ) the wild-type $\alpha 1 \mathrm{C}$ with a $\mathrm{HCN} 2$ mutant lacking the $\mathrm{NH}_{2}$ terminus [HCN2(-N)] and 2) the wild-type $\mathrm{HCN} 2$ with an $\alpha 1 \mathrm{C}$ mutant lacking the IQ motif in the $\mathrm{COOH}$ terminus $[\alpha 1 \mathrm{C} \Delta(1623-1666)]$ (8). Figure $4 A$ shows that the inactivation of $\alpha 1 \mathrm{C} / \mathrm{HCN} 2$ (solid line) was eliminated by either HCN2(-N) or $\alpha 1 C \Delta(1623-1666)$ (shaded lines).

We next examined how $\alpha 1 \mathrm{C}$ and $\mathrm{HCN} 2$ channel mutants interact in the presence of the $\alpha 2 \delta$ subunit. Figure $4 B$ shows that compared with the $\alpha 1 \mathrm{C} / \mathrm{HCN} 2 / \alpha 2 \delta$ channel inactivation (solid line), both $\alpha 1 \mathrm{C} / \mathrm{HCN} 2(-\mathrm{N}) / \alpha 2 \delta$ and $\alpha 1 \mathrm{C} \Delta(1623-1666) /$ $\mathrm{HCN} 2 / \alpha 2 \delta$ channels dramatically slowed the inactivation pro-
Fig. 4. $\alpha 1 \mathrm{C} / \mathrm{HCN} 2$ interaction requires $\mathrm{NH}_{2}$ terminus of HCN2 and IQ motif of $\alpha 1 C$. $A$ : $\alpha 1 \mathrm{C} /$ $\mathrm{HCN} 2$ channel inactivation for wild-type (solid line), $\alpha 1 \mathrm{C} \Delta(1623-1666) / \mathrm{HCN} 2$ (shaded line), and $\alpha 1 \mathrm{C} /$ HCN2(-N) channels (shaded line). $B: \alpha 1 \mathrm{C} / \mathrm{HCN} 2 /$ $\alpha 2 \delta$ channel inactivation for wild-type (solid line), $\alpha 1 \mathrm{C} \Delta(1623-1666) / \mathrm{HCN} 2 / \alpha 2 \delta$ (shaded line), and $\alpha 1 \mathrm{C} / \mathrm{HCN} 2(-\mathrm{N}) / \alpha 2 \delta$ channels (shaded line). $C$ : inactivation of $\alpha 1 \mathrm{C} / \alpha 2 \delta / \beta 1 / \mathrm{HCN} 2$ (solid line), $\alpha 1 \mathrm{C}(1623-1666) / \alpha 2 \delta / \beta 1 / \mathrm{HCN} 2$ (shaded line), and $\alpha 1 \mathrm{C} / \alpha 2 \delta / \beta 1 / \mathrm{HCN} 2(-\mathrm{N})$ (shaded line). $D$ : inactivation time constants measured at $+10 \mathrm{mV}$ for showing the significant role of $\mathrm{HCN} 2(-\mathrm{N})[\alpha 1 \mathrm{C} /$ $\mathrm{HCN} 2 / \alpha 2 \delta, \alpha 1 \mathrm{C} / \mathrm{HCN} 2(-\mathrm{N}) / \alpha 2 \delta, \alpha 1 \mathrm{C} / \mathrm{HCN} 2 / \alpha 2 \delta /$ $\mathrm{CaM}_{(\mathrm{WT})}$, and $\alpha 1 \mathrm{C} / \mathrm{HCN} 2 / \alpha 2 \delta / \mathrm{CaM}_{(1,2,3,4)}$; solid bars] and the significant role of $\alpha 1 \mathrm{C} \Delta(1623$ 1666) $[\alpha 1 \mathrm{C} 1905 \mathrm{x} / \mathrm{HCN} 2, \alpha 1 \mathrm{C} 1905 \mathrm{x} / \mathrm{HCN} 2 / \alpha 2 \delta$, $\alpha 1 \mathrm{C} 1905 \mathrm{x} / \mathrm{HCN} 2 / \alpha 2 \delta / \mathrm{CaM}(\mathrm{WT})$, and $\alpha 1 \mathrm{C} \Delta(1623-$ $1666) / H C N 2 / \alpha 2 \delta$; shaded bars]. Results are expressed as means \pm SE. $* P<0.0001$, statistically significant difference compared with $\alpha 1 \mathrm{C} / \mathrm{HCN} 2 /$ $\alpha 2 \delta ; P=0.07$ for $\alpha 1 \mathrm{C} / \mathrm{HCN} 2 / \alpha 2 \delta / \mathrm{CaM}_{(\mathrm{WT})}$ and $P=0.08$ for $\alpha 1 \mathrm{C} / \mathrm{HCN} 2 / \alpha 2 \delta / \mathrm{CaM}_{(123,4)}(n=$ $6-12) . \& P<0.0001$, statistically significant difference compared with $\alpha 1 \mathrm{C} / \mathrm{HCN} 2 / \alpha 2 \delta$ or $\alpha 1 \mathrm{C} 1905 \mathrm{x} /$ $\mathrm{HCN} 2 / \alpha 2 \delta ; P=0.006$ for $\alpha 1 \mathrm{C} 1905 \mathrm{x} / \mathrm{HCN} 2 / \alpha 2 \delta /$ $\mathrm{CaM}_{(\mathrm{WT})}$ and $P=0.0003$ for $\alpha 1 \mathrm{C} \Delta(1623-1666) /$ $\mathrm{HCN} 2 / \alpha 2 \delta(n=5-12)$. E: inactivation of $\alpha 1 \mathrm{C} /$ $\mathrm{HCN} 2 / \alpha 2 \delta$ channel in the presence of $\mathrm{Ba}^{2+}$ (solid line) and $\mathrm{Ca}^{2+}$ (shaded line), respectively.
A

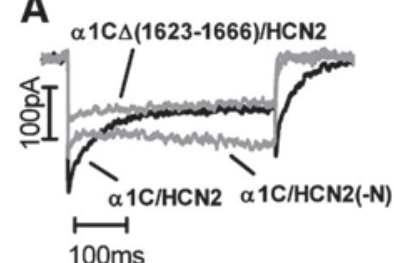

B

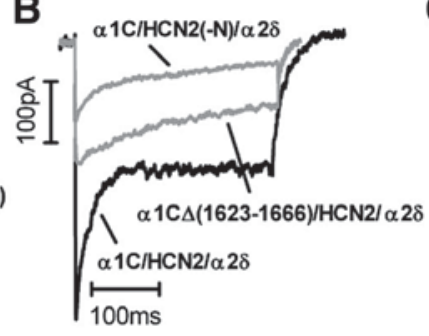

C $\alpha 1 \mathrm{C} \Delta(1623-1666) / \alpha 2 \delta / \beta 1 / \mathrm{HCN} 2$

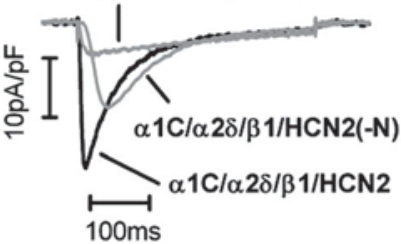

D

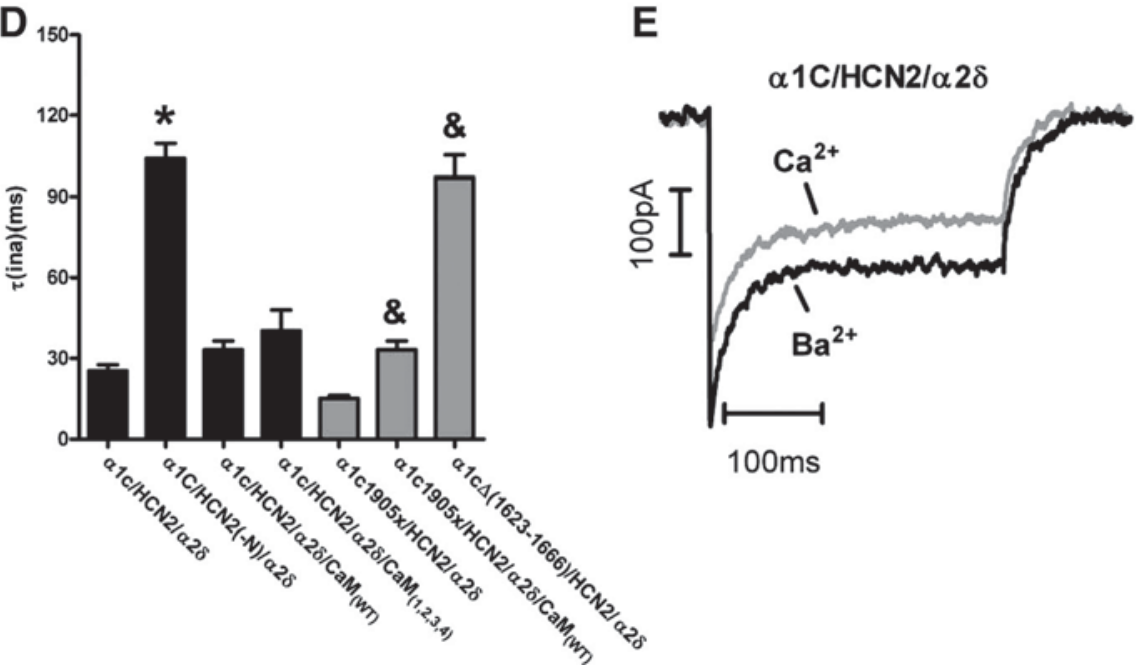


cess (shaded lines). We also observed biphasic inactivation of $\alpha 1 \mathrm{C} / \mathrm{HCN} 2(-\mathrm{N}) / \alpha 2 \delta$ (Fig. $4 B$, shaded line) with its first fast inactivation phase close to the wild type but with a second phase almost linear that cannot be fitted by an exponential function.

We finally studied the effects of HCN2 and $\alpha 1 \mathrm{C}$ mutants on $\alpha 1 \mathrm{C} / \mathrm{HCN} 2$ inactivation in the presence of both $\alpha 2 \delta$ - and $\beta 1$-subunits. Compared with the wild-type $\alpha 1 \mathrm{C} / \alpha 2 \delta / \beta 1 / \mathrm{HCN} 2$ channel (solid line), the significant slowing of $\alpha 1 \mathrm{C} / \mathrm{HCN} 2$ channel inactivation by either HCN2(-N) or $\alpha 1 \mathrm{C} \Delta(1623-1666)$ (shaded lines) still persisted in the presence of $\alpha 2 \delta$ - and $\beta 1$-subunits (Fig. $4 C$ ). At $+10 \mathrm{mV}$, the time constants of $I_{\mathrm{Ba}}$ inactivation were $72.5 \pm 5.6 \mathrm{~ms}$ for $\alpha 1 \mathrm{C} / \alpha 2 \delta / \beta 1 / \mathrm{HCN} 2,108 \pm 13$ $\mathrm{ms}$ for $\alpha 1 \mathrm{C} / \alpha 2 \delta / \beta 1 / \mathrm{HCN} 2(-\mathrm{N})$, and $498 \pm 43 \mathrm{~ms}$ for $\alpha 1 \mathrm{C} \Delta(1623-1666) / \alpha 2 \delta / \beta 1 / \mathrm{HCN} 2$, respectively $(n=5-7)$ (Fig. 4D). Together, these results suggest that the $\mathrm{NH}_{2}$ terminus of HCN2 and the IQ motif of $\alpha 1 \mathrm{C}$ are the structural elements required for the inactivation of the $\alpha 1 \mathrm{C} / \mathrm{HCN} 2$ channel.

Relative roles of $\mathrm{CaM}, \alpha 2 \delta$, and $\beta 1$ in the inactivation of $\alpha 1$ C/HCN2 channels. To examine the relative contribution of $\alpha 2 \delta$ and $\mathrm{CaM}$ to the inactivation of $\alpha 1 \mathrm{C} / \mathrm{HCN} 2$ channels, we compared the inactivation time constants for different combinations of $\alpha 1 \mathrm{C}, \mathrm{HCN} 2, \alpha 2 \delta$, and $\mathrm{CaM}$, including their respective mutants, except for $\alpha 1 \mathrm{C} 1905 \mathrm{x} / \mathrm{HCN} 2 . \alpha 1 \mathrm{C} 1905 \mathrm{x}$ is a distal $\mathrm{COOH}$-terminal truncated form of $\alpha 1 \mathrm{C}$, which is used as a control for $\alpha 1 \mathrm{C} \Delta(1623-1666)$ function (8). We found that $\alpha 1 C \Delta(1623-1666)$ and HCN2(-N) are the two most significant structural motifs that contribute to the inactivation of $\alpha 1 \mathrm{C} /$ HCN2 channels independently of $\alpha 2 \delta$ (Fig. 4D). We also found, surprisingly, that overexpression of $\mathrm{CaM}_{(\mathrm{WT})}$ did not accelerate (as one would expect), but slowed, the inactivation of $\alpha 1 \mathrm{C} / \mathrm{HCN} 2 / \alpha 2 \delta$ or $\alpha 1 \mathrm{C} 1905 \mathrm{x} / \mathrm{HCN} 2 / \alpha 2 \delta$ channels (Fig. $4 D$ ).

In the absence of $\alpha 2 \delta$, there was a distinct difference between CDI and VDI for $\alpha 1 \mathrm{C} / \mathrm{HCN} 2$ channels (Fig. $2 B$ ). In the presence of $\alpha 2 \delta$, however, the difference between CDI and VDI disappeared (Fig. 4E). Combined with the diminished difference between CDI and VDI for $\alpha 1 \mathrm{C} / \alpha 2 \delta / \beta 1 / \mathrm{HCN} 2$ (Fig. $1 B$ ), the results provide additional evidence for a major role of $\alpha 2 \delta$ in the inactivation of $\alpha 1 \mathrm{C} / \mathrm{HCN} 2$ channels. In comparing Fig. 4, $C$ and $E, \beta 1$ helped complete the $I_{\mathrm{Ba}}$ inactivation of $\alpha 1 \mathrm{C} / \mathrm{HCN} 2 / \alpha 2 \delta$ channels, which are consistent with the results shown in Fig. $1 B$.

Association of $\alpha 1 C$ and HCN2 channel proteins in HEK-293 and hippocampus. Whether the interaction of $\alpha 1 \mathrm{C}$ and HCN2 is mediated by $\mathrm{CaM}$ (for $\alpha 1 \mathrm{C} / \mathrm{HCN} 2$ channels) or by $\delta$-subunit (for $\alpha 1 \mathrm{C} / \mathrm{HCN} 2 / \alpha 2 \delta$ channels), $\alpha 1 \mathrm{C}$ and HCN2 channel proteins should be physically close. Fig $5 A$ shows that in HEK293 cells expressing $\alpha 1 \mathrm{C}$ and HCN2, $\alpha 1 \mathrm{C}$ signal could indeed be detected with an $\alpha 1 \mathrm{C}$ antibody after the samples were immunoprecipitated by an HCN2 antibody (left lane). Immunoblots using an $\alpha 1 \mathrm{C}$ antibody for cells expressing $\alpha 1 \mathrm{C} / \alpha 2 \delta$ and cells with no cDNA transfection (NT) served as positive and negative controls, respectively. Figure $5 B$ shows that HCN2 signal could also be detected with its antibody after the samples were immunoprecipitated by an $\alpha 1 \mathrm{C}$ antibody. Figure $5 C$ shows that in HEK-293 cells expressing $\alpha 1 \mathrm{C}$ and a hemagglutinin (HA)-tagged HCN2 cDNA plasmid, the $\alpha 1 \mathrm{C}$ signal was detected using an anti- $\alpha 1 \mathrm{C}$ antibody after the sample was immunoprecipitated with an anti-HA antibody (Fig. 5C, top). The signal was, however, undetected in cells expressing $\alpha 1 \mathrm{C} \Delta(1623-1666) / \mathrm{HCN} 2$ or $\mathrm{HCN} 2(-\mathrm{N}) / \alpha 1 \mathrm{C}$ (Fig. 5C, top).
On the other hand, HCN2 bands were seen with an HA antibody in the sample immunoprecipitated using an antibody specific to $\alpha 1 \mathrm{C}$ (Fig. 5C, bottom). However, the signals were very weak in cells expressing $\alpha 1 \mathrm{C} \Delta(1623-1666) / \mathrm{HCN} 2$ and undetectable in cells expressing $\mathrm{HCN} 2(-\mathrm{N}) / \alpha 1 \mathrm{C}$ (Fig. $5 C$, bottom).

To investigate the relative contribution of $\alpha 2 \delta$ and CaM to the association of $\alpha 1 \mathrm{C}$ and HCN2, Fig. $5 D$ shows the coimmunoprecipitation results in HEK-293 cells overexpressing the respective combinations of cDNAs. In the presence of $\alpha 2 \delta$, the association of $\alpha 1 \mathrm{C}$ and $\mathrm{HCN} 2$ was not significantly enhanced by $\mathrm{CaM}$ compared with $\mathrm{CaM}_{(1,2,3,4)}$. In the absence of $\alpha 2 \delta$, however, the association of $\alpha 1 \mathrm{C}$ and $\mathrm{HCN} 2$ was more significantly enhanced by $\mathrm{CaM}$ than by $\mathrm{CaM}_{(1,2,3,4)}$, as evidenced by much stronger signals detected by either $\alpha 1 \mathrm{C}$ antibody (top) or HCN2 antibody (bottom). Although one should not expect a linear correlation between the protein chemistry result and whole cell patch-clamp data due to the technical difference, these results are consistent with the functional roles of $\alpha 2 \delta$ and $\mathrm{CaM}$ in the interaction of $\alpha 1 \mathrm{C}$ and $\mathrm{HCN} 2$ (Fig. 3).

To demonstrate the existence of such an association in native tissues, we chose rat hippocampus as a test model due to the technical feasibility and rich expression of LTCC (21) and HCN2 (19) in the hippocampus. We used fresh rat hippocampus tissues for the abundant amount of proteins to facilitate the coimmunoprecipitation experiments. In Fig. $5 E$, the $\alpha 1 \mathrm{C}$ signals were readily detectable when the sample was first immunoprecipitated by an HCN2 antibody (left). On the other hand, the HCN2 band was also evident in the sample immunoprecipitated first by $\alpha 1 \mathrm{C}$ antibody (right). For negative control of coimmunoprecipitation in hippocampal neurons, the samples were immunoprecipitated with a GFP antibody and then blotted by either an HCN2 antibody or an $\alpha 1 \mathrm{C}$ antibody. No expected signals were detected (Fig. $5 F$ ).

Slowing $\mathrm{Ca}^{2+}$ inactivation by disrupting $\alpha 1 \mathrm{C} / \mathrm{HCN} 2$ association in hippocampus neurons. $\mathrm{NH}_{2}$ terminus of $\mathrm{HCN} 2$ was reported to be essential in the formation of the homo- and heteromeric channels at the plasma membrane (17). Overexpression of $\mathrm{HCN} 2$ lacking $\mathrm{NH}_{2}$ terminus in the hippocampal neurons, in which the endogenous expression levels of both $\alpha 1 \mathrm{C}$ and $\mathrm{HCN} 2$ channel proteins are comparable and the two proteins are associated (Fig. 5E), may disrupt the association. Shown in Fig. 6A, formation of the rat hippocampal neuron network was visualized after 5-6 days in culture. After 24- to 48-h transfection of HCN2(-N) with GFP, the expression of HCN2(-N) was indicated by the fluorescent image (Fig. 6B) compared with the brightfield image of the same region (Fig. $6 A$ ). The fluorescent neurons were subject to the patch-clamp recordings. Shown in Fig. $6 C$, overexpression of HCN2(-N) induced a remarkably slow inactivation component (light shaded line) that was not present in the control neurons or in neurons transfected with the empty vector (solid line, as a transfection control). On the other hand, transfection with the full-length $\mathrm{HCN} 2$ accelerated the $I_{\mathrm{Ba}}$ inactivation (dark shaded line in Fig. $6 C$ ). Currents were verified by verapamil $(10 \mu \mathrm{M})$ blockade (Fig. $6 C$ ). At $+10 \mathrm{mV}$, time constants of $I_{\mathrm{Ba}}$ inactivation were $26.5 \pm 2.9 \mathrm{~ms}(n=9)$ for control hippocampal neurons $(\mathrm{HN}), 35.1 \pm 3.5 \mathrm{~ms}(n=6)$ for $\mathrm{HN}+$ vector, $66.1 \pm 3.3$ $\mathrm{ms}(n=7)$ for $\mathrm{HN}+\mathrm{HCN} 2(-\mathrm{N})$, and $19.0 \pm 2.2 \mathrm{~ms}(n=10)$ for $\mathrm{HN}+\mathrm{HCN} 2$ (Fig. 6D). The altered time constants for channel 

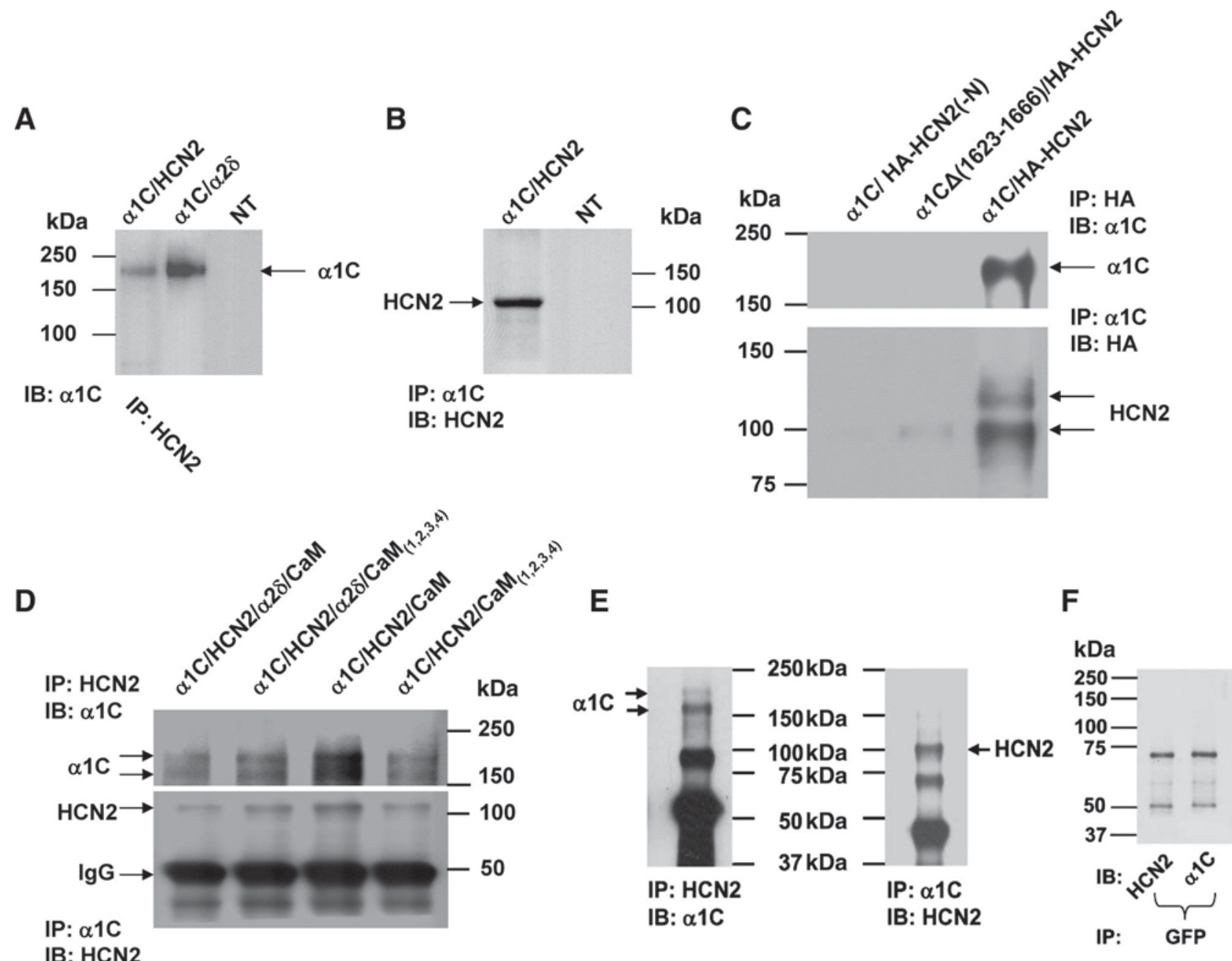

Fig. 5. Association of $\alpha 1 \mathrm{C}$ and HCN2 proteins in HEK-293 cells and in the hippocampus. A: cells expressing $\alpha 1 \mathrm{C}$ and HCN2 were immunoprecipitated (IP) using an HCN2 antibody, followed by signal detection (IB) using an $\alpha 1 \mathrm{C}$ antibody (left lane). Cells expressing $\alpha 1 \mathrm{C}$ and $\alpha 2 \delta$ were blotted by an $\alpha 1 \mathrm{C}$ antibody (middle lane), serving as a positive control. Cells without cDNA transfection (NT) were blotted using an $\alpha 1 \mathrm{C}$ antibody (right lane), serving as a negative control. $B$ : cells expressing $\alpha 1 \mathrm{C}$ and $\mathrm{HCN} 2$ were immunoprecipitated using an $\alpha 1 \mathrm{C}$ antibody, followed by signal detection using an HCN2 antibody. Cells without cDNA transfection served as a negative control. $C$, top: in cells coexpressing $\alpha 1 \mathrm{C}$ and $\mathrm{HA}-\mathrm{HCN} 2, \alpha 1 \mathrm{C}$ signal was detected using an anti- $\alpha 1 \mathrm{C}$ antibody after the sample was immunoprecipitated by a specific anti-hemagglutinin (HA) antibody. Signals were not detected in cells expressing $\mathrm{HCN} 2(-\mathrm{N}) / \alpha 1 \mathrm{C}$ or $\mathrm{HCN} 2 / \alpha 1 \mathrm{C} \Delta(1623-$ 1666). Bottom, in cells coexpressing $\alpha 1 \mathrm{C}$ and HCN2, HCN2 signals were detected by an HA antibody in the sample immunoprecipitated with an $\alpha 1 \mathrm{C}$ antibody. Very weak signal was seen in cells expressing $\mathrm{HCN} 2 / \alpha 1 \mathrm{C} \Delta(1623-1666)$ but not in cells expressing $\mathrm{HCN} 2(-\mathrm{N}) / \alpha 1 \mathrm{C}$. $D$ : effect of CaM and $\alpha 2 \delta$ on association of $\alpha 1 \mathrm{C}$ and HCN2. Top, samples were immunoprecipitated using an HCN2 antibody, followed by signal detection with an $\alpha 1 \mathrm{C}$ antibody. Bottom, samples were immunoprecipitated using an $\alpha 1 \mathrm{C}$ antibody, followed by signal detection with an $\mathrm{HCN} 2$ antibody. IgG bands were included as controls. E: coimmunoprecipitation of $\alpha 1 \mathrm{C}$ and HCN2 in the hippocampus. Left, sample was immunoprecipitated with an HCN2 antibody and blotted by an $\alpha 1 \mathrm{C}$ antibody. Right, sample was immunoprecipitated with an $\alpha 1 \mathrm{C}$ antibody and blotted by an $\mathrm{HCN} 2$ antibody. $F$ : negative control for coimmunoprecipitation in the rat hippocampus. Samples were immunoprecipitated using a green fluorescent protein (GFP) antibody, followed by immunoblots using an HCN2 antibody (left lane) and an $\alpha 1 \mathrm{C}$ antibody (right lane), respectively.

inactivation were marked with statistical significance compared with the control $\mathrm{HN}$ or $\mathrm{HN}$ transfected with vector (Fig. $6 D$ ).

\section{DISCUSSION}

Both $\alpha 1 \mathrm{C}$ - and $\delta$-subunits are transmembrane proteins with $\mathrm{NH}_{2}$ and $\mathrm{COOH}$ tails of $\alpha 1 \mathrm{C}$ and $\mathrm{COOH}$ tail of $\delta$ located intracellularly. $\mathrm{CaM}$ and $\beta 1$-subunit are intracellular proteins attached to the $\alpha 1 \mathrm{C}$ near the inner surface of the membrane. These four proteins constitute the inactivation machinery for the fine control of $\mathrm{Ca}^{2+}$ influx through LTCC in both VDI and CDI $(6,12,15,22)$.

Our previous studies showed possible involvement of HCN in the modulation of myocyte action potential duration (24). In the present work, we evaluated the hypothesis that inactivation of LTCC can be modulated via interaction with $\mathrm{HCN}$ channels under the experimental conditions that prevent $\mathrm{HCN} 2$ channel opening. We showed here that $\mathrm{HCN} 2$ can act as a nonchannel regulatory protein to induce a fast inactivation of $\alpha 1 \mathrm{C}$. To further investigate the possible associated mechanisms involved in the interaction between $\alpha 1 \mathrm{C}$ and $\mathrm{HCN} 2$ channel proteins, we proposed a model shown in Fig. 7. Mechanism 1 represents our current understanding of interactions among $\beta$-subunits, which are bound to the $\alpha 1$-interaction domain (AID), apoCaM, and inactivation motifs in the $\mathrm{COOH}$-terminal region of $\alpha 1 \mathrm{C}$. The majority of this conceptual understanding was provided from studies in either Xenopus oocytes or HEK- 

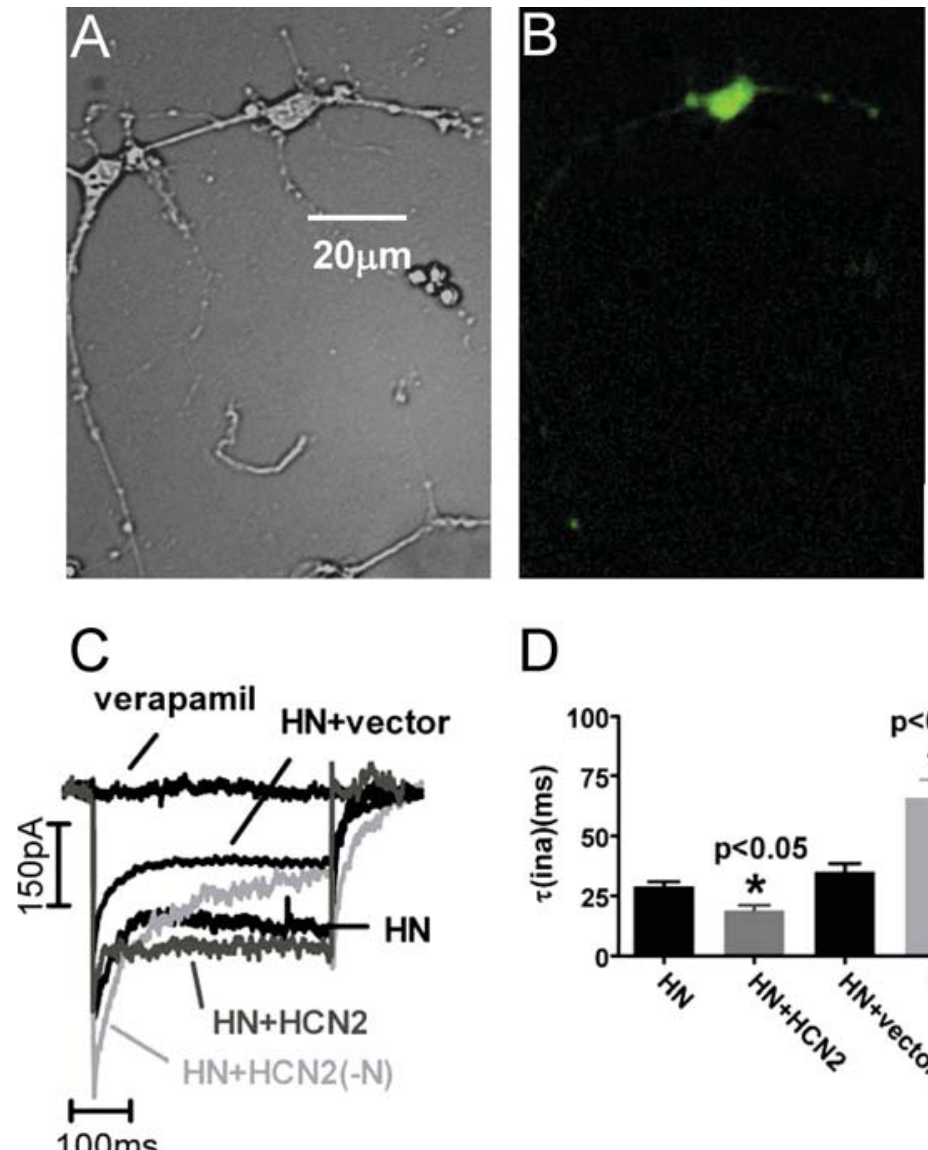

D

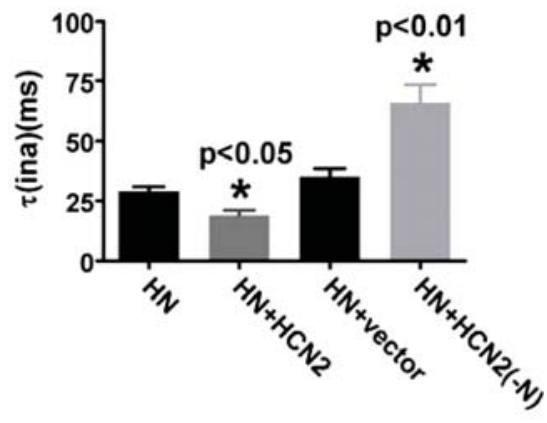

Fig. 6. Altered L-type $\mathrm{Ca}^{2+}$ channel (LTCC) inactivation in rat hippocampal neurons (HN) overexpressing $\mathrm{HCN} 2(-\mathrm{N})$ and the full-length $\mathrm{HCN} 2$. A brightfield image $(A)$ and fluorescence image $(B)$ of the region is shown $48 \mathrm{~h}$ posttransfection of HCN2(-N) or $\mathrm{HCN} 2$ with GFP. $C$ : $\mathrm{Ca}^{2+}$ current inactivation using $\mathrm{Ba}^{2+}$ as a charge carrier in a control neuron and in neurons transfected with the empty vector ( $\mathrm{HN}+$ vector), in a neuron transfected with $\mathrm{HCN} 2(-\mathrm{N})[\mathrm{HN}+\mathrm{HCN} 2(-\mathrm{N})]$, and in a neuron transfected with HCN2 (HN + HCN2). I $\mathrm{Ba}$ currents were recorded at $+10 \mathrm{mV}$. The holding potential was at -90 $\mathrm{mV}$. Pulse duration was $400 \mathrm{~ms}$. P4 protocol was used. The HN + vector trace was moved up by $60 \mathrm{pA}$ for clear illustration of currents under different conditions. $D$ : inactivation time constants in $\mathrm{HN}, \mathrm{HN}$ transfected with $\mathrm{HCN} 2$, $\mathrm{HN}$ transfected with the vector, and $\mathrm{HN}$ transfected with HCN2(-N). Results are expressed as means $\pm \mathrm{SE}$. $P$ values indicate statistical significance compared with either $\mathrm{HN}$ or $\mathrm{HN}+$ vector group.
293 cells coexpressing $\alpha 1 \mathrm{C}$ with $\beta 1$ - and $\alpha 2 \delta$-subunits $(6,15$, $16,20,27,28)$. Our present study revealed several new concepts.

First, in the absence of $\beta 1-$ and $\alpha 2 \delta$-subunits, HCN 2 can induce a faster inactivation of $\alpha 1 \mathrm{C}$ than $\alpha 2 \delta$ as measured by $I_{\mathrm{Ba}}$ (compare Fig. 2, $A$ and $B$ ). This interaction of HCN2 and $\alpha 1 \mathrm{C}$ requires CaM (mechanism 2 in Fig. 7), since two CaM inhibitors (W-7 and TFP) and the dominant negative $\mathrm{CaM}_{(1,2,3,4)}$ all eliminated the inactivation of $\alpha 1 \mathrm{C} / \mathrm{HCN} 2$ channel (Fig. 3, $A$ and $B$ ).
Second, in the presence of the $\alpha 2 \delta$-subunit, HCN2 can still induce fast inactivation of $\alpha 1 \mathrm{C}$. However, the impact of $\mathrm{CaM}$ in affecting the inactivation of $\alpha 1 \mathrm{C} / \mathrm{HCN} 2 / \alpha 2 \delta$ channel is diminished compared with that for $\alpha 1 \mathrm{C} / \mathrm{HCN} 2$ channel, suggesting a prevalent role of the $\alpha 2 \delta$-subunit in $\alpha 1 \mathrm{C}$ and HCN2 interaction (mechanism 3 in Fig. 7).

Third, mutants of $\mathrm{HCN} 2$ (with the $\mathrm{NH}_{2}$ terminus deleted) and $\alpha 1 \mathrm{C}$ (without IQ motif) revealed a critical role of these two structural elements in mediating the fast inactivation of $\alpha 1 \mathrm{C} /$ HCN2 channels (Fig. 4). It is worth noting that the short

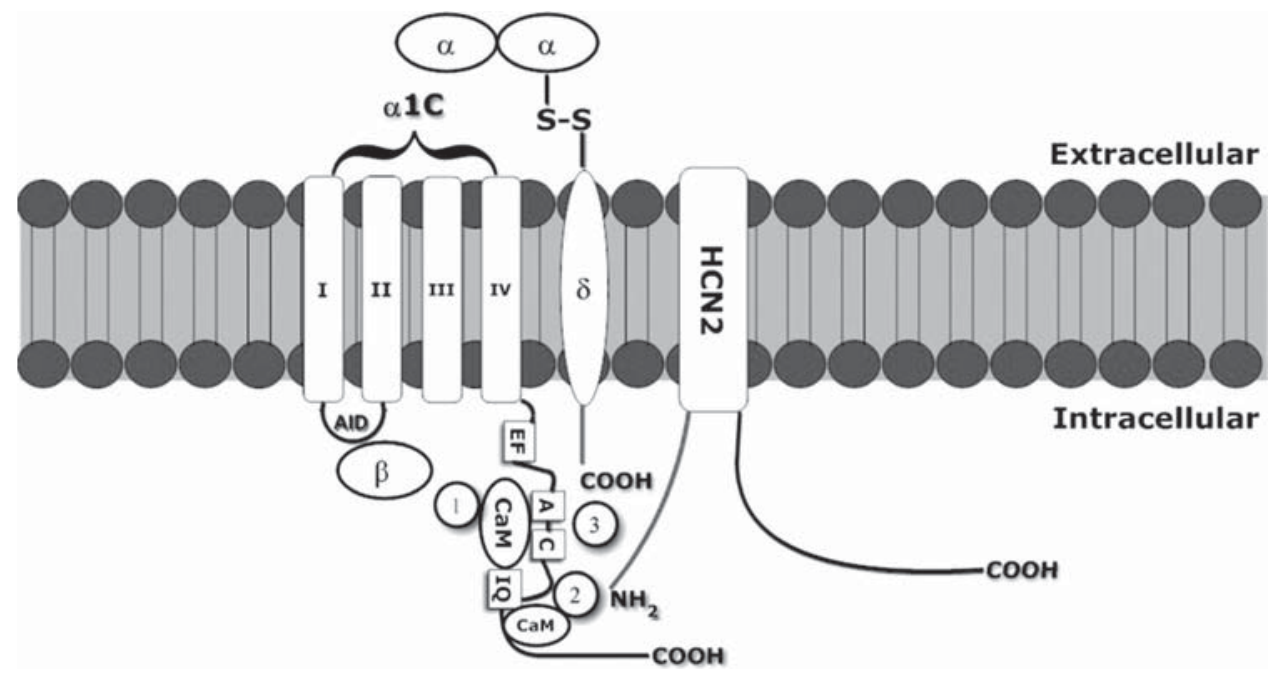

Fig. 7. A schematic model depicting the potential roles of $\mathrm{HCN} 2$ and $\delta$-subunit in the inactivation of LTCC. Mechanism 1 summarizes the mechanisms for CDI and VDI of LTCC. Mechanism 2 proposes a potential CaM-mediated interaction between the $\mathrm{NH}_{2}$ terminus of HCN2 and the IQ motif of $\alpha 1 \mathrm{C}$. Mechanism 3 proposes a potential interaction between the $\mathrm{COOH}$ tail of the $\delta$-subunit and the $\mathrm{NH}_{2}$ terminus of $\mathrm{HCN} 2$ within the $\mathrm{COOH}$-terminal inactivation machinery of $\alpha 1 \mathrm{C}$-subunit. AID, $\alpha 1$-interaction domain. 
$\mathrm{COOH}$ terminus of the $\delta$-subunit is located in the intracellular space. Analysis of amino acids in the $\mathrm{NH}_{2}$ terminus of $\mathrm{HCN} 2$ showed an overall positive charge, which can potentially interact with the negatively charged $\mathrm{COOH}$ terminus of $\delta$-subunit. Although previous studies have suggested a vital role for the $\delta$-subunit in accelerating $\alpha 1 \mathrm{C}$ inactivation $(7,23)$, there is limited mechanistic insight into how this process occurs. Our data suggested that a key contributor to this accelerated inactivation may be a dynamic, coordinated interaction among the $\mathrm{COOH}$ terminus of the $\delta$-subunit, the IQ motif of $\alpha 1 \mathrm{C}$, and the $\mathrm{NH}_{2}$ terminus of HCN2. Alternatively, it is also possible that $\beta 1$-subunit can interfere with the inactivation of $\alpha 1 \mathrm{C} \Delta(1623$ $1666) / \mathrm{HCN} 2 / \alpha 2 \delta$ and $\alpha 1 \mathrm{C} / \alpha 2 \delta / \mathrm{HCN} 2(-\mathrm{N}$ ), as well (Fig. $4 C$ ). This may be a logical possibility, since the $\beta 1$-subunit is attached to the linker of I-II domains and has been reported to interact with the IQ motif either directly or possibly through the actions of CaM (26). Our results for $\mathrm{HCN} 2(-\mathrm{N})$ and $\alpha 1 \mathrm{C} \Delta(1623-1666)$ (Fig. 5C) also are consistent with previous reports that the $\mathrm{NH}_{2}$ terminus of HCN2 and IQ motif of $\alpha 1 \mathrm{C}$ are important structural elements for the respective channel surface expression $(8,17)$. Thus it is possible that the altered channel trafficking leading to the reduced surface expression of either $\mathrm{HCN} 2$ or $\alpha 1 \mathrm{C}$ provides a potential mechanism for the roles of $\mathrm{NH}_{2}$ terminus of $\mathrm{HCN} 2$ and IQ motif of $\alpha 1 \mathrm{C}$ in the interaction of the two channels.

Finally, we have demonstrated that an interaction of $\alpha 1 \mathrm{C}$ and HCN2 is present in native tissues, as evidenced by association of the two channel proteins in the hippocampus and dramatic slowing of $I_{\mathrm{Ba}}$ in hippocampal neurons overexpressing $\mathrm{HCN} 2(-\mathrm{N})$ (Fig. 6). It should be noted that the currents we recorded in hippocampal neurons are not pure LTCCs, since Nand $\mathrm{P} / \mathrm{Q}$-calcium channels are present in hippocampal neurons, and verapamil can also inhibit $\mathrm{N}$ - and P/Q-type calcium currents (5). Nevertheless, the fact that overexpression of HCN2(-N) mutant in HNs induced a significant slowing of inactivation supports the hypothesis that dynamic interaction between $\alpha 1 \mathrm{C}$ and HCN2 exists in the native tissue.

Unusual features of $\alpha 1 C / H C N 2$ and $\alpha 1 C / H C N 2 / \alpha 2 \delta$ channel inactivation. Dissecting VDI and CDI often has been carried out by using $\mathrm{Ba}^{2+}$ and $\mathrm{Ca}^{2+}$, respectively $(12,15,22$, 28 ), yielding intriguing results. Not only is CDI about $6-10$ times faster than VDI $(12,13)$, but CDI also can come back to the closed state whereas VDI cannot. The CDI index, measured by the residual fraction of the currents that remains at the end of the test pulse, is much smaller for $\mathrm{Ca}^{2+}$ than for $\mathrm{Ba}^{2+}(6,12$, $15,28)$. Interestingly, the speed of VDI can be increased to that of CDI if the IQ motif is deleted (28). Furthermore, a single mutation of I to A or E in the IQ motif could eliminate the difference in CDI index for $\mathrm{Ca}^{2+}$ and $\mathrm{Ba}^{2+}$ (27). Recently, using a fluorescence resonance energy transfer (FRET) twohybrid mapping technique, preassociation of apoCaM and a 73 -amino acid segment containing IQ motif was found to be critical for the slow VDI (6). Disruption of this preassociation significantly accelerated the VDI and removed the difference between CDI and VDI (6).

Both $\alpha 1 \mathrm{C} / \mathrm{HCN} 2$ and $\alpha 1 \mathrm{C} / \mathrm{HCN} 2 / \alpha 2 \delta$ channels have exhibited VDI rates that are faster than that reported following the disruption of preassociation of IQ motif and apoCaM (6). Our data suggest that $\mathrm{HCN} 2$, via its $\mathrm{NH}_{2}$ terminus, can interrupt the preassociation of apoCaM with the IQ motif of $\alpha 1 \mathrm{C}$ and that this may contribute to the accelerated VDI inactivation. The requirement of $\mathrm{CaM}$ for $\alpha 1 \mathrm{C} / \mathrm{HCN} 2$ inactivation in the absence of $\alpha 2 \delta$ subunit is supported by the facts that inhibiting CaM activity using either pharmacological inhibitors or overexpression of $\mathrm{CaM}_{(1,2,3,4)}$ all eliminated the inactivation of $\alpha 1 \mathrm{C} /$ HCN2 (Fig. 3, $A$ and $B$ ). On the other hand, $\alpha 1 \mathrm{C} / \mathrm{HCN} 2 / \alpha 2 \delta$ channel inactivation is largely independent of CaM (Fig. 3, C and $D$ ). One possible scenario for this difference may be that the conformational change of $\mathrm{CaM}$ resulted from the interaction between the IQ motif and HCN2 is different from that resulted from the interaction between the IQ motif and HCN2/ $\alpha 2 \delta$. The interaction between the IQ motif and HCN2 may lead $\mathrm{CaM}$ to undergo a conformational change that exposes its hydrophobic activation site to the CaM blockers, whereas the interaction between the IQ motif and $\mathrm{HCN} 2 / \alpha 2 \delta$ may induce a different CaM conformational change that does not make its blockers accessible to the activation site. This possibility would explain the fast VDI of $\alpha 1 \mathrm{C} / \mathrm{HCN} 2 / \alpha 2 \delta$ channel (solid lines in Fig. 3, $C$ and $D$ ), the insensitivity of $\mathrm{W}-7$ and TFP to the $\alpha 1 \mathrm{C} / \mathrm{HCN} 2 / \alpha 2 \delta$ currents (Fig. $3 C$ ), and the lack of a difference between CDI and VDI for the $\alpha 1 \mathrm{C} / \mathrm{HCN} 2 / \alpha 2 \delta$ channel (Fig. 4E).

Potential implications of $\alpha 1 C$ and HCN2 interaction. In this study, although we have presented evidence demonstrating the interaction of $\alpha 1 \mathrm{C}$-subunit (Cav1.2) with $\mathrm{HCN} 2$, interactions of this type might also occur in other types of $\mathrm{Ca}^{2+}$ channels that undergo CDI, such as Cav2 channels, as a common mechanism. Use of $\mathrm{CaM}$ as the key element for $\mathrm{Ca}^{2+}$ channel inactivation has been reported for Cav1 and Cav2 channels (13).

Increased $\mathrm{Ca}^{2+}$ influx is associated with a number of neuronal disorders, including neuropathic pain (1). Gabapentin, a GABA analog, is an antiepileptic drug that has been used recently to relieve neuropathic pain and is believed to bind the $\alpha 2 \delta 1$-subunit, leading to decreased $\mathrm{Ca}^{2+}$ influx $(1,9)$. The mechanism of how gabapentin binding of $\alpha 2 \delta 1$-subunit decreased $\mathrm{Ca}^{2+}$ influx remains elusive, since the gabapentin action is acute (1). The $\alpha 2 \delta$-subunits have been known to enhance the surface expression of $\alpha 1 \mathrm{C}$ and $\mathrm{Ca}^{2+}$ current amplitude $(7,23)$. A recent study demonstrated that chronic treatment with gabapentin was associated with a reduction in the surface expression of $\alpha 2 \delta 2$ and Cav2.1 subunits (9). On the other hand, the $\alpha 2 \delta$-subunits also promote inactivation of Cav1.2 ( $\alpha 1 \mathrm{C}$ ) or Cav2.2 (N-type) (23), and inhibition of the $\alpha 2 \delta$ subunit should, in principle, slow the $\alpha 1 \mathrm{C}$ inactivation, resulting in an increased $\mathrm{Ca}^{2+}$ influx. The interaction of $\alpha 1 \mathrm{C}$ with $\mathrm{HCN} 2$ may provide an alternative mechanism to prevent an increase in $\mathrm{Ca}^{2+}$ influx under the conditions in which the $\alpha 2 \delta$-subunit expression is reduced.

Study limitations. Although we report for the first time a novel and potentially important mechanism for modulation of $\mathrm{Ca}^{2+}$ current inactivation by $\mathrm{HCN} 2$ channel, there are many unanswered questions. For instance, we noticed that the activation of $I_{\mathrm{CaL}}$ also seems to be altered by $\mathrm{HCN} 2$ (e.g., Fig. $1 F$ ). In addition, whether $\mathrm{HCN} 2$ may also modulate other $\mathrm{Ca}^{2+}$ channels that share similar inactivation machinery to $\alpha 1 \mathrm{C}$ is unknown. More importantly, under various physiological and especially pathological conditions, the importance of interaction between HCN2 and LTCC requires further investigation, since the expression levels of $\mathrm{HCN} 2, \alpha 1 \mathrm{C}$, and $\alpha 2 \delta 1$ are altered. 


\section{ACKNOWLEDGMENTS}

We are grateful for the generous gifts of $\mathrm{CaM}_{(\mathrm{WT})}$ and $\mathrm{CaM}_{(1,2,3,4)}$ from $\mathrm{Dr}$. John P. Adelman (Ohio Health Sciences University) and Dr. Wayne Chen (University of Calgary), $\alpha 1 \mathrm{C}$ from Dr. Diane Lipscombe (Brown University), $\mathrm{HCN} 2\left(-\mathrm{NH}_{2}\right)$ from Dr. Michael Sanguinetti (University of Utah), and $\alpha 1 C \Delta(1623-1666)$ and $\alpha 1 C 1905 x$ from Dr. Tianyan Gao (University of Texas, Galveston). We also appreciate the stable HEK-293 cell line expressing $\alpha 2 \delta$ and $\beta 1$-subunits from Dr. Richard Tsien (Stanford University).

\section{GRANTS}

This work was supported by National Institutes of Health (NIH) Grant HL075023 and the Office of Research and Graduate Programs/Health Sciences Center at West Virginia University (to H.-G. Yu). Microscope experiments and image analysis were performed in the West Virginia University Microscope Imaging Facility, supported in part by the Mary Babb Randolph Cancer Center and NIH Grant P20 RR016440.

\section{DISCLOSURES}

No conflicts of interest are declared by the author(s).

\section{REFERENCES}

1. Altier C, Zamponi GW. Targeting $\mathrm{Ca}^{2+}$ channels to treat pain: T-type versus N-type. Trends Pharmacol Sci 25: 465-470, 2004.

2. Arikkath J, Campbell KP. Auxiliary subunits: essential components of the voltage-gated calcium channel complex. Curr Opin Neurobiol 13: 298-307, 2003.

3. Cens T, Rousset M, Leyris JP, Fesquet P, Charnet P. Voltage- and calcium-dependent inactivation in high voltage-gated $\mathrm{Ca}^{2+}$ channels. Prog Biophys Mol Biol 90: 104-117, 2006.

4. DiFrancesco D. Pacemaker mechanisms in cardiac tissue. Annu Rev Physiol 55: 455-472, 1993.

5. Dobrev D, Milde AS, Andreas K, Ravens U. The effects of verapamil and diltiazem on $\mathrm{N}-$, $\mathrm{P}$ - and Q-type calcium channels mediating dopamine release in rat striatum. Br J Pharmacol 127: 576-582, 1999.

6. Erickson MG, Liang H, Mori MX, Yue DT. FRET two-hybrid mapping reveals function and location of L-type $\mathrm{Ca}^{2+}$ channel CaM preassociation. Neuron 39: 97-107, 2003.

7. Felix R, Gurnett CA, De Waard M, Campbell KP. Dissection of functional domains of the voltage-dependent $\mathrm{Ca}^{2+}$ channel alpha2delta subunit. J Neurosci 17: 6884-6891, 1997.

8. Gao T, Bunemann M, Gerhardstein BL, Ma H, Hosey MM. Role of the $\mathrm{C}$ terminus of the alpha $1 \mathrm{C}\left(\mathrm{Ca}_{\mathrm{v}} 1.2\right)$ subunit in membrane targeting of cardiac L-type calcium channels. J Biol Chem 275: 25436-25444, 2000.

9. Hendrich J, Van Minh AT, Heblich F, Nieto-Rostro M, Watschinger K, Striessnig J, Wratten J, Davies A, Dolphin AC. Pharmacological disruption of calcium channel trafficking by the alpha2delta ligand gabapentin. Proc Natl Acad Sci USA 105: 3628-3633, 2008.

10. Hidaka H, Sasaki Y, Tanaka T, Endo T, Ohno S, Fujii Y, Nagata T. $N$-(6-aminohexyl)-5-chloro-1-naphthalenesulfonamide, a calmodulin antagonist, inhibits cell proliferation. Proc Natl Acad Sci USA 78: 43544357, 1981

11. Huang J, Huang A, Zhang Q, Lin YC, Yu HG. Novel mechanism for suppression of hyperpolarization-activated cyclic nucleotide-gated pacemaker channels by receptor-like tyrosine phosphatase- $\alpha$. J Biol Chem 283: 29912-29919, 2008.
12. Kim J, Ghosh S, Nunziato DA, Pitt GS. Identification of the components controlling inactivation of voltage-gated $\mathrm{Ca}^{2+}$ channels. Neuron 41: 745$754,2004$.

13. Liang H, DeMaria CD, Erickson MG, Mori MX, Alseikhan BA, Yue DT. Unified mechanisms of $\mathrm{Ca}^{2+}$ regulation across the $\mathrm{Ca}^{2+}$ channel family. Neuron 39: 951-960, 2003.

14. Osawa M, Swindells MB, Tanikawa J, Tanaka T, Mase T, Furuya T, Ikura M. Solution structure of calmodulin-W-7 complex: the basis of diversity in molecular recognition. J Mol Biol 276: 165-176, 1998.

15. Peterson BZ, DeMaria CD, Adelman JP, Yue DT. Calmodulin is the $\mathrm{Ca}^{2+}$ sensor for $\mathrm{Ca}^{2+}$-dependent inactivation of L-type calcium channels. Neuron 22: 549-558, 1999.

16. Pitt GS, Zuhlke RD, Hudmon A, Schulman H, Reuter H, Tsien RW. Molecular basis of calmodulin tethering and $\mathrm{Ca}^{2+}$-dependent inactivation of L-type $\mathrm{Ca}^{2+}$ channels. J Biol Chem 276: 30794-30802, 2001.

17. Proenza C, Tran N, Angoli D, Zahynacz K, Balcar P, Accili EA. Different roles for the cyclic nucleotide binding domain and amino terminus in assembly and expression of hyperpolarization-activated, cyclic nucleotide-gated channels. J Biol Chem 277: 29634-29642, 2002.

18. Reinhart PH, Taylor WM, Bygrave FL. Trifluoperazine, an inhibitor of calmodulin action, antagonises phenylephrine-induced metabolic responses and mitochondrial calcium fluxes in liver. FEBS Lett 120: 71-74, 1980.

19. Santoro B, Chen S, Luthi A, Pavlidis P, Shumyatsky GP, Tibbs GR, Siegelbaum SA. Molecular and functional heterogeneity of hyperpolarization-activated pacemaker channels in the mouse CNS. J Neurosci 20: $5264-5275,2000$.

20. Wang HG, George MS, Kim J, Wang C, Pitt GS. $\mathrm{Ca}^{2+} / \mathrm{calmodulin}$ regulates trafficking of Cav1.2 $\mathrm{Ca}^{2+}$ channels in cultured hippocampal neurons. J Neurosci 27: 9086-9093, 2007.

21. Westenbroek RE, Ahlijanian MK, Catterall WA. Clustering of L-type $\mathrm{Ca}^{2+}$ channels at the base of major dendrites in hippocampal pyramidal neurons. Nature 347: 281-284, 1990.

22. Xia XM, Fakler B, Rivard A, Wayman G, Johnson-Pais T, Keen JE, Ishii T, Hirschberg B, Bond CT, Lutsenko S, Maylie J, Adelman JP. Mechanism of calcium gating in small-conductance calcium-activated potassium channels. Nature 395: 503-507, 1998.

23. Yasuda T, Chen L, Barr W, McRory JE, Lewis RJ, Adams DJ, Zamponi GW. Auxiliary subunit regulation of high-voltage activated calcium channels expressed in mammalian cells. Eur J Neurosci 20: 1-13, 2004

24. Yu X, Chen XW, Zhou P, Yao L, Liu T, Zhang B, Li Y, Zheng H, Zheng LH, Zhang CX, Bruce I, Ge JB, Wang SQ, Hu ZA, Yu HGc-CA, Zhou Z. Calcium influx through $I_{\mathrm{f}}$ channels in rat ventricular myocytes. Am J Physiol Cell Physiol 292: C1147-C1155, 2007.

25. Yu X, Duan KL, Shang CF, Yu HG, Zhou Z. Calcium influx through hyperpolarization-activated cation channels ( $I_{\mathrm{h}}$ channels) contributes to activity-evoked neuronal secretion. Proc Natl Acad Sci USA 101: 10511056, 2004.

26. Zhang R, Dzhura I, Grueter CE, Thiel W, Colbran RJ, Anderson ME. A dynamic alpha-beta inter-subunit agonist signaling complex is a novel feedback mechanism for regulating L-type $\mathrm{Ca}^{2+}$ channel opening. FASEB $J$ 19: 1573-1575, 2005.

27. Zuhlke RD, Pitt GS, Deisseroth K, Tsien RW, Reuter H. Calmodulin supports both inactivation and facilitation of L-type calcium channels. Nature 399: 159-162, 1999.

28. Zuhlke RD, Reuter H. $\mathrm{Ca}^{2+}$-sensitive inactivation of $\mathrm{L}$-type $\mathrm{Ca}^{2+}$ channels depends on multiple cytoplasmic amino acid sequences of the alpha1C subunit. Proc Natl Acad Sci USA 95: 3287-3294, 1998. 